\title{
Gold Nanoparticle-Based Surface Plasmon Mediated Surface- Enhanced Raman Scattering and Energy Transfer Platforms for Sensing Applications
}

\author{
Ming Li \\ West Virginia University
}

Follow this and additional works at: https://researchrepository.wvu.edu/etd

\author{
Recommended Citation \\ Li, Ming, "Gold Nanoparticle-Based Surface Plasmon Mediated Surface-Enhanced Raman Scattering and \\ Energy Transfer Platforms for Sensing Applications" (2012). Graduate Theses, Dissertations, and Problem \\ Reports. 3602. \\ https://researchrepository.wvu.edu/etd/3602
}

This Dissertation is protected by copyright and/or related rights. It has been brought to you by the The Research Repository @ WVU with permission from the rights-holder(s). You are free to use this Dissertation in any way that is permitted by the copyright and related rights legislation that applies to your use. For other uses you must obtain permission from the rights-holder(s) directly, unless additional rights are indicated by a Creative Commons license in the record and/ or on the work itself. This Dissertation has been accepted for inclusion in WVU Graduate Theses, Dissertations, and Problem Reports collection by an authorized administrator of The Research Repository @ WVU. For more information, please contact researchrepository@mail.wvu.edu. 


\title{
Gold Nanoparticle-Based Surface Plasmon Mediated Surface-Enhanced Raman Scattering and Energy Transfer Platforms for Sensing Applications
}

\author{
Ming Li \\ Dissertation submitted to the \\ College of Engineering and Mineral Resources \\ at West Virginia University \\ in partial fulfillment of the requirements \\ for the degree of \\ Doctor of Philosophy \\ In \\ Mechanical Engineering
}

Nianqiang Wu, Ph.D., Chair

Larry A. Hornak, Ph.D.

Ever J. Barbero, Ph.D.

Xiaodong Shi, Ph.D.

Edward M. Sabolsky, Ph.D.

Daneesh Olivia Simien, Ph.D.

Department of Mechanical and Aerospace Engineering

West Virginia University

Morgantown, West Virginia

2012

Key words: Surface plasmon resonance; Surface-enhanced Raman scattering;

Fluorescence; Quantum dots; Gold nanoparticle; Resonance energy transfer

Copyright 2012 Ming Li 


\section{ABSTRACT \\ Gold Nanoparticle-Based Surface Plasmon Mediated Surface-Enhanced Raman Scattering and Energy Transfer Platforms for Sensing Applications}

Ming Li

Gold nanoparticles (GNPs) exhibit unique optical properties, depending on the particle size, geometrical shape, and medium refractive index and interparticle interactions, etc. Among these optical properties, localized surface plasmon resonance (LSPR) has significant effects on the electromagnetic field around the GNPs. The LSPR-induced electromagnetic field enhancement is beneficial to the surface-enhanced Raman scattering (SERS) and energy transfer processes. This dissertation deals with the effects of LSPR on SERS and energy transfer between CdSe/ZnS quantum dots and GNPs. Specifically, the research aims to gain better understanding of (i) the electromagnetic enhancement induced by charge transfer from GNP to molecules, (ii) the SERS in various shaped gold nanostructures, (iii) the energy transfer from quantum dots (QDs) to GNPs, and (iv) SERS- and energy transfer-based sensing platforms for detection of chemical species and biomolecules.

The SERS of two different types of molecules on GNPs has been investigated. It has been found that the aromatic molecules such as $p$-mercaptobenzenoic acid (MBA) exhibit stronger SERS activity than the linear-chain molecules such as 3-mercaptopropionic acid (MPA) and Lcysteine (Cys). The difference in the SERS activity is attributed to the distinct electronic structures among these molecules. The electron transfer from GNPs to MBA can occur under laser excitation. The transferred electron can effectively strengthen the electromagnetic field around GNPs, leading to electromagnetic enhancement of SERS.

Furthermore, the SERS in different shaped gold nanostructures (gold nanospheres (GSPs), nanorods (GRDs) and nanostars (GSTs)) has been investigated. GSTs show the highest SERS enhancement. Three-dimensional finite-difference time domain (FDTD) method has been used to simulate the electric field distribution. It is demonstrated that the electric field can be concentrated around two ends of GRDs and these tips of GSTs, and the GSTs show the highest 
maximum electric field intensity under both excitations of $532 \mathrm{~nm}$ and $785 \mathrm{~nm}$. It is suggested that the shape of gold nanostructures governs the SERS difference among GSPs, GRDs and GSTs. In addition, gold@malachite green isothiocyanate(MGITC)@ $\mathrm{SiO}_{2} \quad$ sandwiched nanostructures have been prepared. $\mathrm{SiO}_{2}$ encapsulation not only improves the colloidal and LSPR stability but also endows excellent reproducibility of SERS signal due to the prevention of MGITC leaking. It is demonstrated that the GST@MGITC@ $\mathrm{SiO}_{2}$ can be used for monitoring of DNA hybridization and for detection of adenosine triphosphate (ATP) with high sensitivity.

Energy transfer between the CdSe/ZnS QDs and GNPs was investigated. The $3 \mathrm{~nm}$ GNPs without observable LSPR absorption quench the fluorescence emission of the QDs following a nanometal surface energy transfer (NSET) while the large sized GNPs quench the fluorescence emission via the Förster resonance energy transfer (FRET) with a $1 / d^{6}$ distance dependence. The quenching efficiency of fluorescence emission increases with the increase of particle size. It is suggested that the involvement of LSPR and the increasing spectral overlap between the LSPR band and the fluorescence emission spectra are responsible for the enhanced energy transfer from $\mathrm{CdSe} / \mathrm{ZnS}$ quantum dots to GNPs.

An ultra-sensitive fluorescent sensor based on the quantum dot-DNA-gold nanoparticle ensemble has been developed for detection of $\mathrm{Hg}$ (II). When $\mathrm{Hg}$ (II) ions are present in the aqueous solution containing the DNA-conjugated quantum dots (QDs) and GNPs, the QDs and the GNPs are brought into the close proximity, which enables the nanometal surface energy transfer (NSET), quenching the fluorescence emission of the QDs. This nanosensor exhibits a limit of detection of $0.4 \mathrm{ppb}$ and $1.2 \mathrm{ppb}$ toward $\mathrm{Hg}$ (II) in the buffer solution and in the river water, respectively. 


\section{ACKNOWLEDGEMENTS}

I would like to express my gratitude to all those who contributes toward the completion of my study, and supports me with strength and knowledge throughout the Ph.D. study. It would have not been possible to complete my Ph.D. study without their help, encouragement and fun.

I am thankful to my advisor, Dr. Nianqiang Wu for providing supports, encouragement and academic guidance. His invaluable discussions inspire me to understand the experimental results and overcome the difficulties. I have learned a lot about scientific research and life from him.

I appreciate my thanks to Dr. Xiaodong Shi and Qiaoyi Wang in his group for invaluable suggestions and kindly offer of DNA. I also would like to devote my gratitude to my committee members, Dr. Larry A. Hornak, Dr. Ever J. Barbero, Dr. Edward M. Sabolsky and Dr. Daneesh Olivia Simien for their guidance and suggestions.

It has been my pleasure to work with all members-Jin Wang, Mingjia Zhi, Fanke Meng, Scott K. Cushing, Chengcheng Xiang, Jiangtian Li, Honglei Gou, Rui Li, Jinlong Yan, Savan Suri and Anveeksh Koneru, in Dr. Nianqiang Wu's group past and present for their help.

Many thanks go to WVU shared facility staff-Dr. Kolin S. Brown, Dr. Marcela Redigolo, Dr. Weiqiang Ding and Mr. Harley Hart for their help.

This work was supported by an NSF grant (CBET-0754405). The resource and facilities used were partially supported by an NSF RII grant (EPS 1003907) and a Research Challenge Grant from the State of West Virginia (EPS08-01), the West Virginia University Research Corporation and the West Virginia EPSCoR Office. 


\section{TABLE OF CONTENTS}

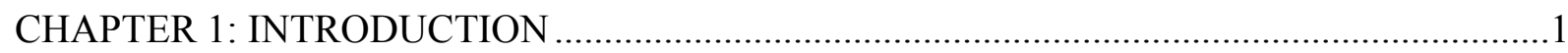

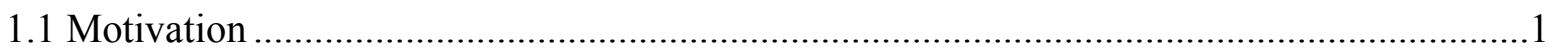

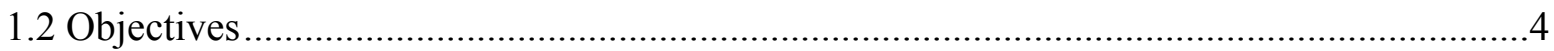

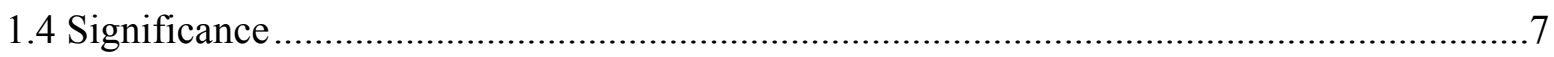

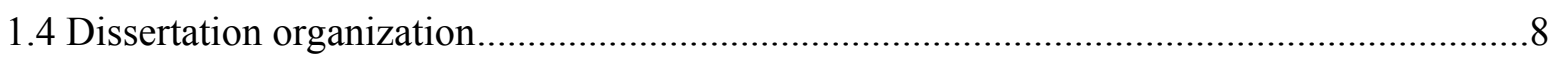

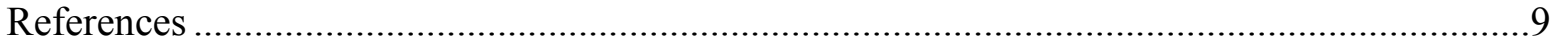

CHAPTER 2: BACKGROUND AND LITERATURE OVERVIEW ……................................11

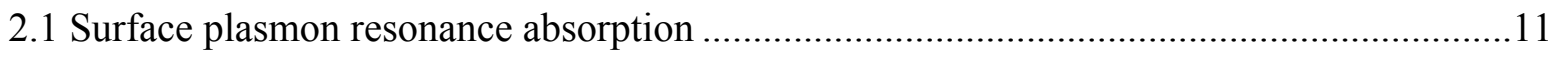

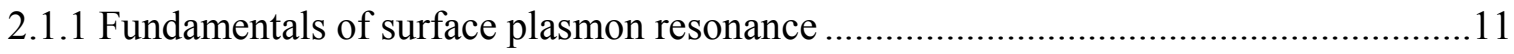

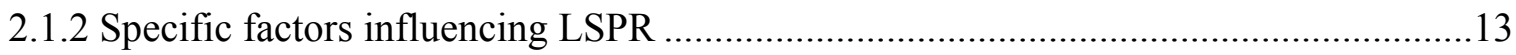

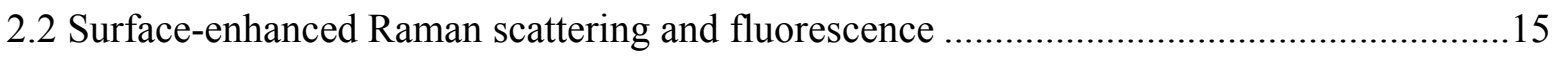

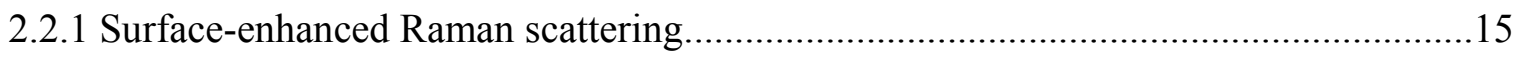

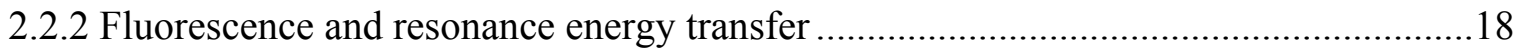

2.3 Localized surface plasmon resonance in SERS and RET ................................................21

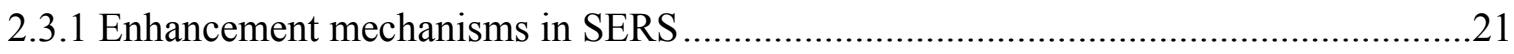

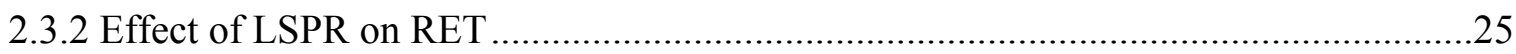

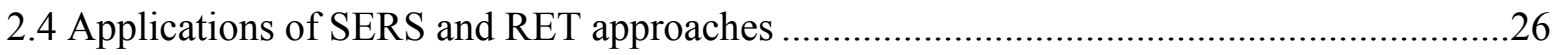

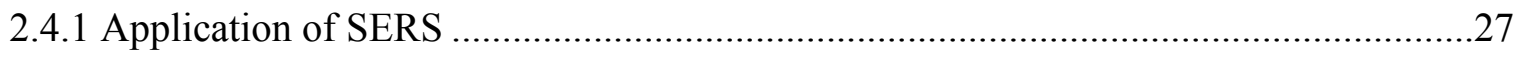

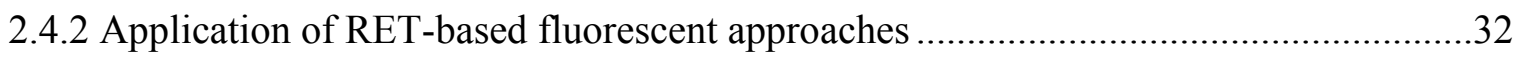

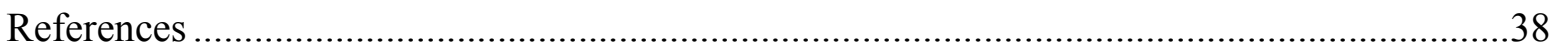

CHAPTER 3: CHARGE TRANSFER INDUCED ELECTROMAGENTIC ENHANCEMENT

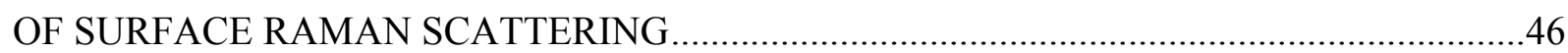

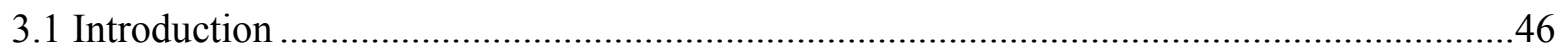

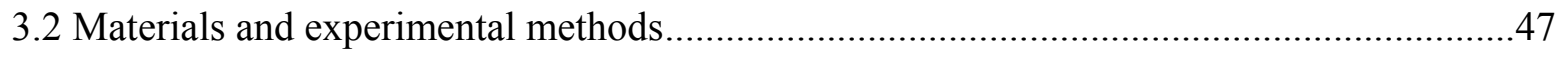

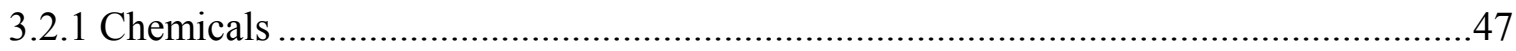

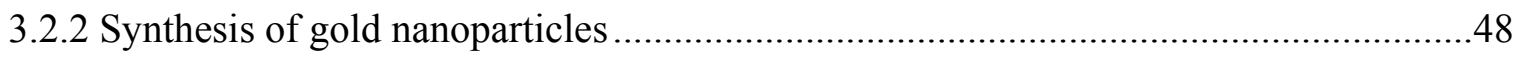

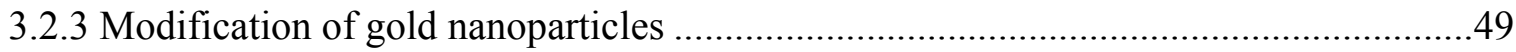

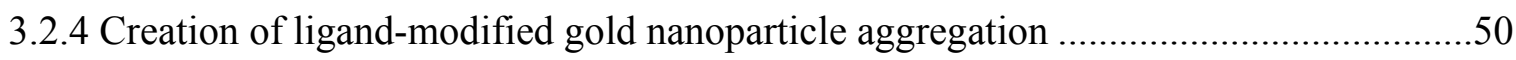

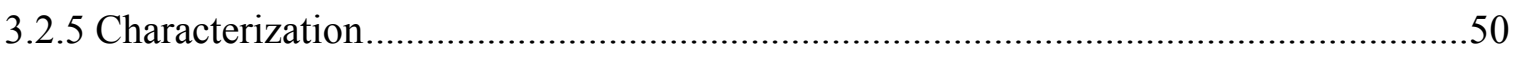

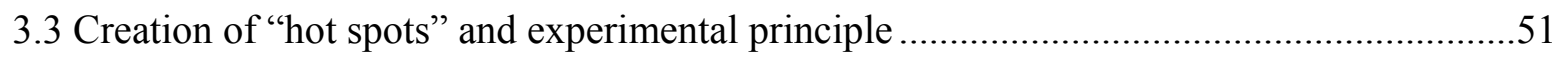

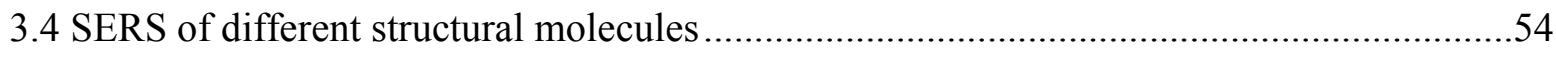

3.5 SERS in different thiophenol to 3-mercaptopropionic acid ratios .....................................54 
3.6 SERS in various thiophenol amounts .56

3.7 SERS in gold nanoparticles with various LSPR absorptions ........................................57

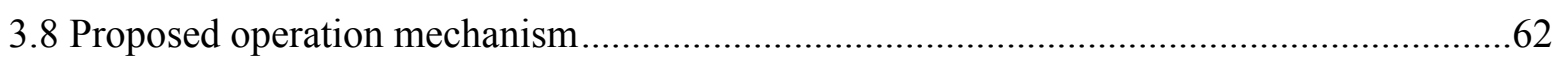

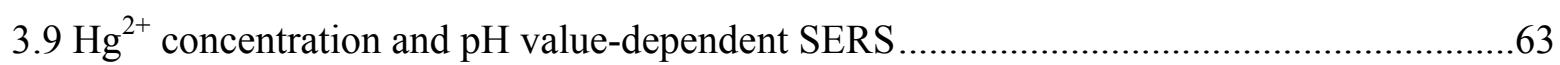

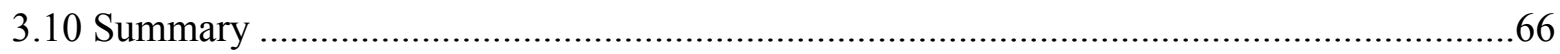

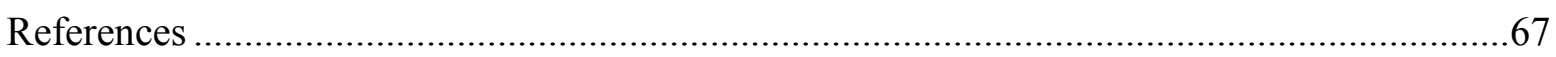

CHAPTER 4: SURFACE-ENHANCED RAMAN SCATTERING IN VARIOUS SHAPED

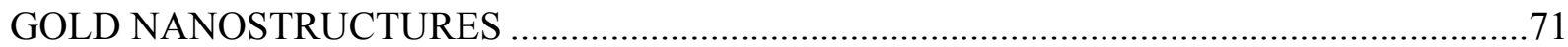

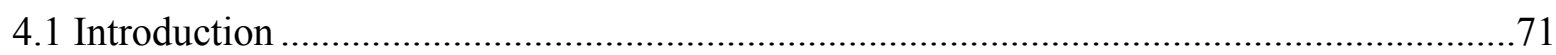

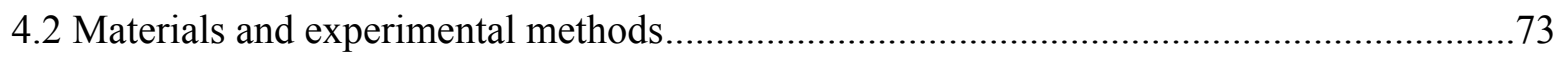

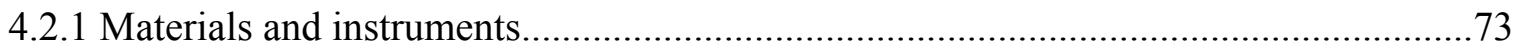

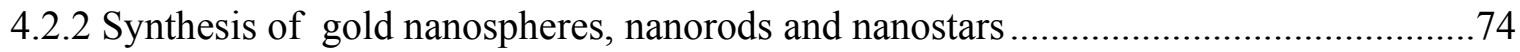

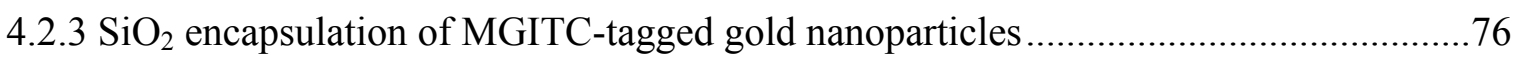

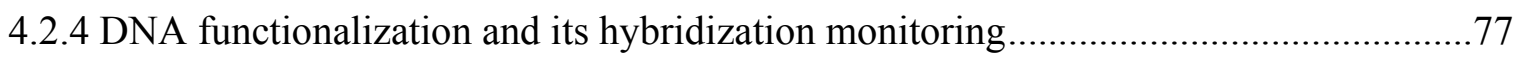

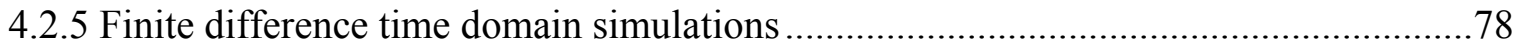

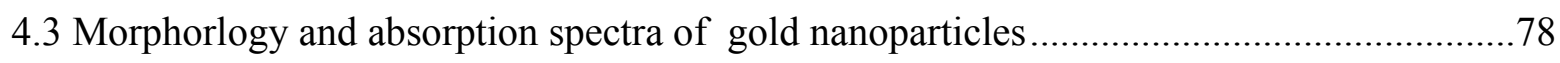

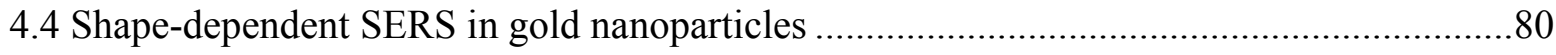

4.5 Electric field distribution and theoretical SERS enhancement factor ...............................83

$4.6 \mathrm{SiO}_{2}$ encapsulation of MGITC-tagged gold nanoparticles..............................................86

4.7 SERS of gold@MGITC@ $\mathrm{SiO}_{2}$ composite particles ............................................................89

4.8 Effects of solvents and salt concentrations on stability..................................................92

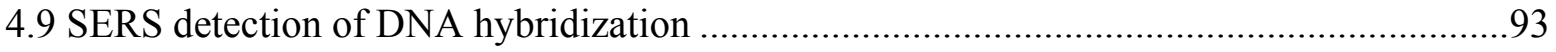

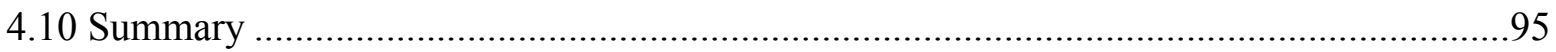

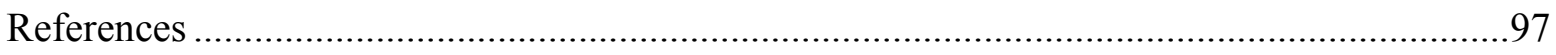

CHAPTER 5: SERS DETECTION OF ATP BASED ON GOLD NANOSTAR@RAMANREPORTER@SILICA SANDWICHED NANOPARTICLES .................................................101

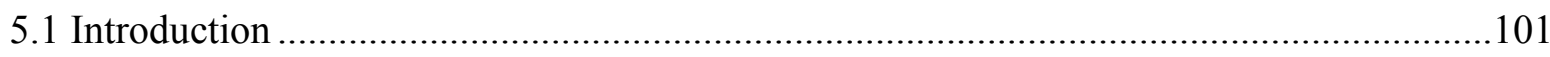

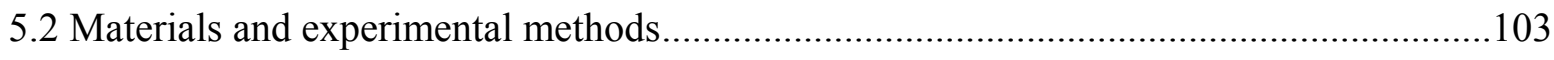

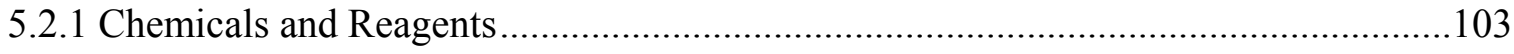

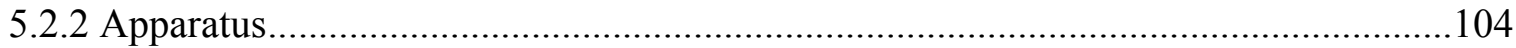

5.2.3 DNA functionalization of gold nanostar@MGITC@SiO2 ...........................................104

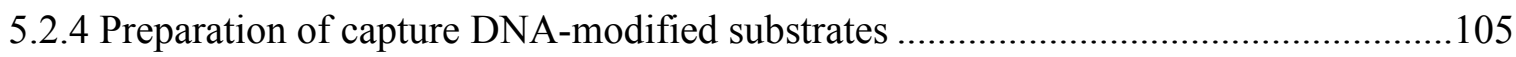


5.2.5 Assembly of the SERS Sensor and its application for ATP Detection

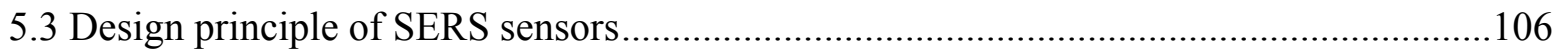

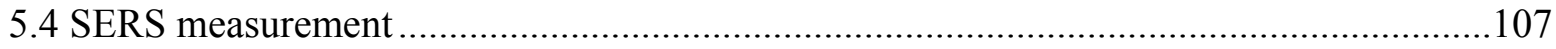

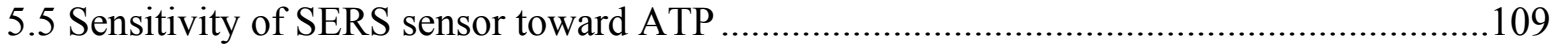

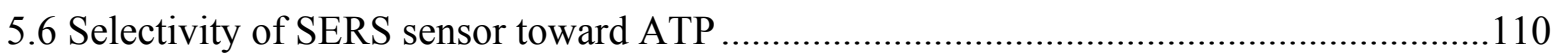

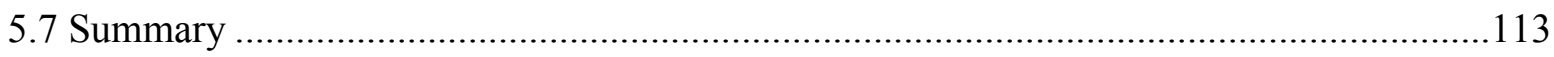

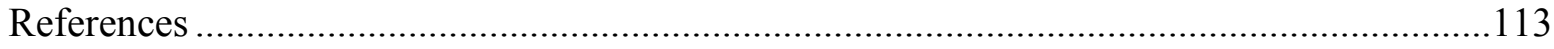

CHAPTER 6: LOCALIZED SURFACE PLASMON-MEDIATED ENERGY TRANSFER BETWEEN QUANTUM DOTS AND GOLD NANOPARTICLES .........................................117

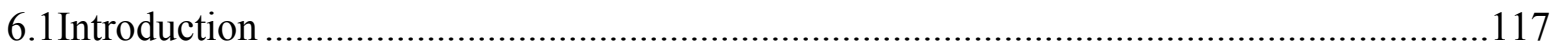

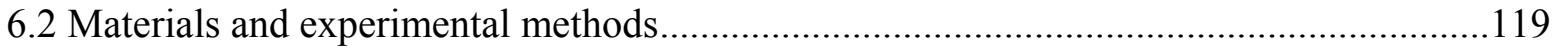

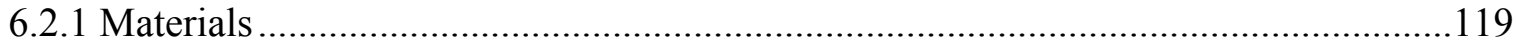

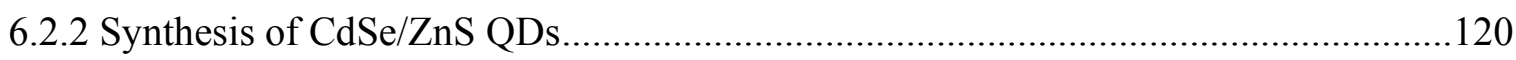

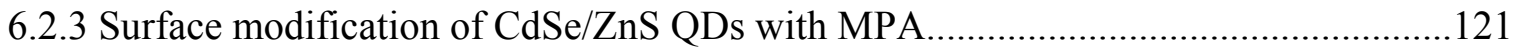

6.2.4 Synthesis of DNA-modified CdSe/ZnS QDs and gold nanoparticles .........................121

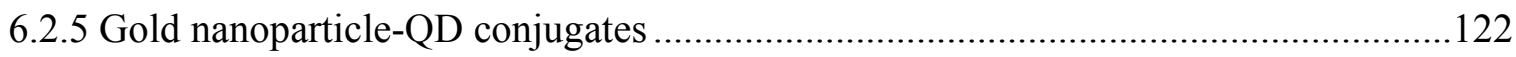

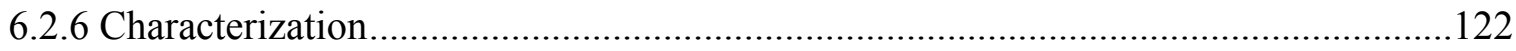

6.3 Characterization and size-tunable optical properties of QDs..........................................123

6.4 Preparation of water-soluble QDs and its surface functionalization ................................126

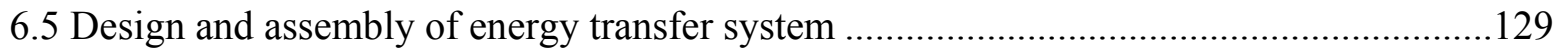

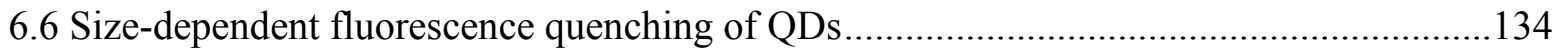

6.7 Energy transfer mechanism in different sized gold systems .........................................136

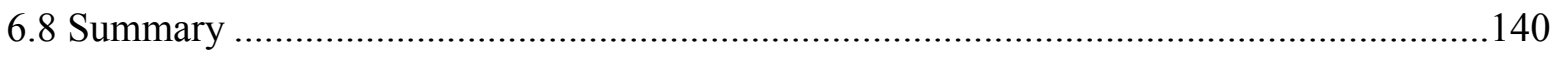

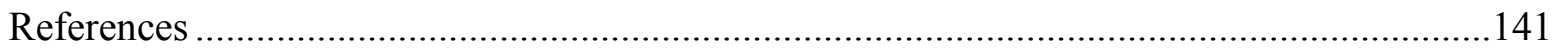

CHAPTER 7: MERCURY(II) DETECTION BASED ON ENERGY TRANSFER BETWEEN

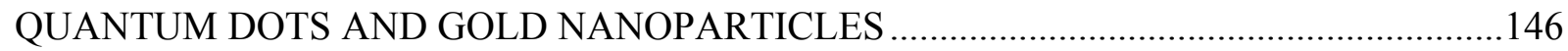

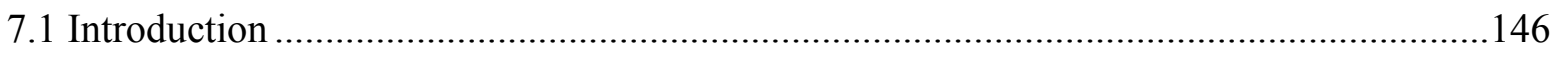

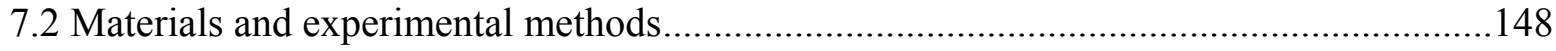

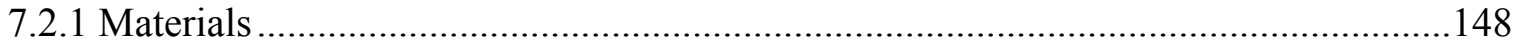

7.2.2 Synthesis of DNA-QD conjugates and DNA-gold conjugates...................................149

7.2.3 Assembly of QD-DNA-gold ensemble and $\mathrm{Hg}^{2+}$ detection ......................................150

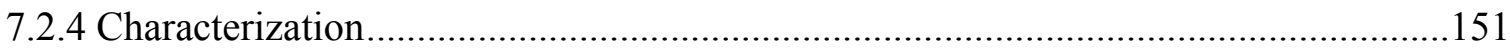

7.3 Design of energy transfer-based sensors and its operation principle .............................151 


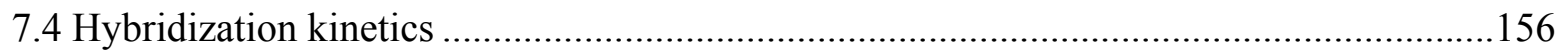

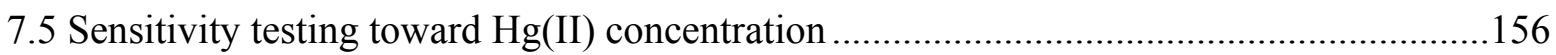

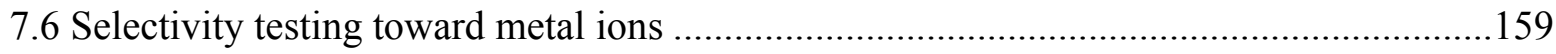

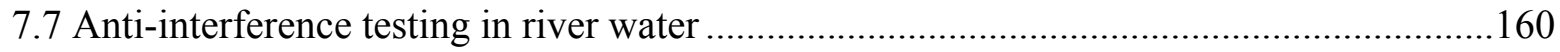

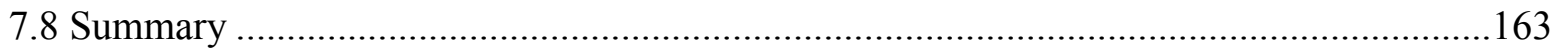

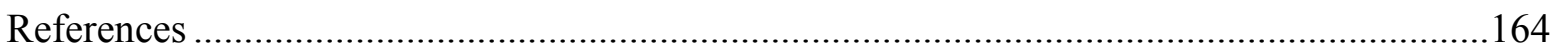

CHAPTER 8: CONCLUSIONS AND REMARKS ON FUTURE RESEARCH.....................167

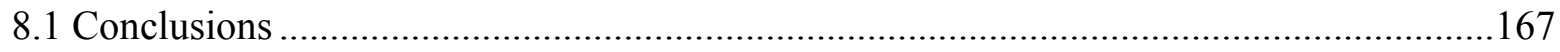

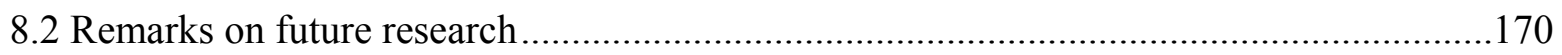

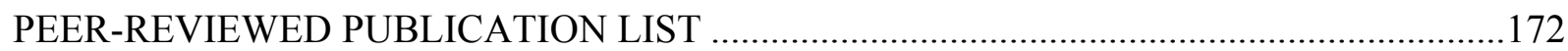




\section{LIST OF TABLES}

Page \#

Table 4.1 Assignments of Selected Raman bands of MGITC in SERS spectra 82

Table 4.2 Experimental and calculated average SERS enhancement factors

Table 5.1 Assignments of Selected Raman bands of MGITC in SERS spectra 


\section{LIST OF FIGURES}

Page \#

Figure 2.1 Schematic illustration of (a) an electromagnetic radiation with a metal nanosphere. A dipole is induced, which oscillates in phase with the electric field of the incoming light. (b) Transverse and longitudinal oscillations of electrons in a metal nanorod [3].

Figure 2.2 Schematic operation principle of Raman spectroscopy in comparison with infrared spectroscopy and Rayleigh scattering.

Figure 2.3 Jablonski diagram illustrating the excitation and emission process of a molecule.

Figure 2.4 Absorption and fluorescence spectra of Rhodamine $6 \mathrm{G}$ in ethanol.

Figure 2.5 Schematic illustration of electromagnetic enhancement in surfaceenhanced Raman scattering of a molecule on a gold or silver film on nanosphere.

Figure 2.6 Schematic illustration of the charge transfer mechanism in a SERS process. (a) The laser energy directly excites the SERS process in resonance with an electronic transition of metal-molecules complex, and the metal disturbs the electronic structure of molecule and thus changes the polarizability; $(b, c)$ the electron transfer indirectly occurs through the metal.

Figure 3.1 (a) Molecular structures of ligands used. (b) Schematic illustration of "hot spots" formed by the coordination chemistry between divalent ion (e.g. $\mathrm{Hg}^{2+}$ ) and carboxylic group.

Figure 3.2 (a) FTIR of pure $p$-mercaptobenzoic acid, $p$-mercaptobenzoic acidmodified gold nanoparticles with $15 \mathrm{~nm}$ in diameter before and after $\mathrm{Hg}^{2+}$ addition. TEM images of $p$-mercaptobenzoic acid-modified gold nanoparticles (b) before and (c) after $\mathrm{Hg}^{2+}$ addition.

Figure 3.3 (a) UV-visible absorption spectra and (b) SERS spectra obtained from the aqueous solution of surface-functionalized gold nanoparticles (15 $\mathrm{nm}$ ) before and after $\mathrm{Hg}^{2+}$ addition. (6 $\mathrm{nM}$ gold nanoparticles, $0.2 \mathrm{mM}$ ligand, $5 \mathrm{mM} \mathrm{Hg}^{2+}$ and $\mathrm{pH}$ value=6.3).

Figure 3.4 Schematic aggregation of the mixed ligand-modified gold nanopartilces in the presence of $\mathrm{Hg}^{2+}$.

Figure 3.5 (a) SERS spectra of solutions containing different thiophenol/3mercaptopropionic acid ratios on $15 \mathrm{~nm}$ gold nanoparticles. (b) The intensity of SERS peak (at $1457 \mathrm{~cm}^{-1}$ ) as a function of the thiophenol/3mercaptopropionic acid molar ratio. (6 $\mathrm{nM}$ gold nanoparticles, $0.2 \mathrm{mM}$ ligands (TP+MPA), $5 \mathrm{mM} \mathrm{Hg}^{2+}$ and $\mathrm{pH}=6.3$ ).

Figure 3.6 (a) SERS spectra of the mixture of thiophenol and 3-mercaptopropoinic acid adsorbed on the $15 \mathrm{~nm}$ sized gold aggregate. The thiophenol molar 
amount increased but the 3-mercaptopropoinic acid molar amount was kept constant (83 molecules with respect to each gold nanoparticle) (6 $\mathrm{nM}$ gold nanoparticles, $5 \mathrm{mM} \mathrm{Hg}^{2+}, 50 \mathrm{~mW}$ laser power, $100 \mathrm{~s}$ of acquisition time).

Figure 3.7 (a) UV-visible absorption spectra of $p$-mercaptobenzoic acid-modified gold nanoparticles with 3,15 and $80 \mathrm{~nm}$ in diameter in the absence and presence of $\mathrm{Hg}^{2+}$. TEM images of (b) $3 \mathrm{~nm}$, (c) $15 \mathrm{~nm}$ and (d) $80 \mathrm{~nm}$ gold nanoparticles.

Figure 3.8 (a) SERS spectra of 3-mercaptopropionic acid- or $p$-mercaptobenzoic acid-modified gold nanoparticles with different particle sizes in the absence and presence of $\mathrm{Hg}^{2+}$. (b) SERS spectra in various thiophenol/3-mercaptopropionic acid ratios adsorbed on $3 \mathrm{~nm}$ gold nanoparticles and (c) their corresponding peak intensity at $1457 \mathrm{~cm}^{-1}$ as a function of thiophenol/3-mercaptopropionic acid ratio. (d) SERS spectra in various thiophenol amounts adsorbed on $3 \mathrm{~nm}$ gold nanoparticles and (e) their corresponding peak intensity at $1457 \mathrm{~cm}^{-1}$ as a function of thiophenol amount.

Figure 3.9 Schematic illustration of (a) charge transfer between gold nanoparticles and adsorbed molecules, proposed operation mechanism of charge transfer-induced EM enhancement (b) between $3 \mathrm{~nm}$ gold nanoparticles and 3-mercaptopropionic acid, (c) between $3 \mathrm{~nm}$ gold nanoparticles and the mixed thiophenol/3-mercaptopropionic acid, and (d) between gold nanoparticles with strong LSPR absorption and the mixed thiophenol/3mercaptopropionic acid.

Figure 3.10 (a) UV-visible absorption spectra, (b) optical photos, (c) SERS spectra of p-mercaptobenzoic acid-modified gold nanoparticles $(15 \mathrm{~nm})$ in the presence of various $\mathrm{Hg}^{2+}$ concentrations, and (d) the $881 \mathrm{~cm}^{-1}$ and 1457 $\mathrm{cm}^{-1}$ peak intensities as a function of $\mathrm{Hg}^{2+}$ concentration.

Figure 3.11 (a) UV-visible absorption spectra, (b) optical photos, (c) SERS spectra of $p$-mercaptobenzoic acid-modified gold nanoparticles $(15 \mathrm{~nm})$ under various $\mathrm{pH}$ values, and (d) the $881 \mathrm{~cm}^{-1}$ and $1457 \mathrm{~cm}^{-1}$ peak intensities as a function of $\mathrm{pH}$ value.

Figure 4.1 TEM images of as-made gold nanospheres, gold nanorods and gold nanostars.

Figure 4.2 UV-visible absorption spectra of gold nanospheres, gold nanorods and gold nanostars.

Figure 4.3 SERS spectra of MGITC $\left(1.25 \times 10^{-7} \mathrm{M}\right)$ in $6 \mathrm{nM}$ gold nanospheres, nanorods and nanostars at $532 \mathrm{~nm}$ laser (laser power: $50 \mathrm{~mW}$, integration time: $10 \mathrm{~s}$ ) and $785 \mathrm{~nm}$ laser (laser power: $120 \mathrm{~mW}$, integration time: $10 \mathrm{~s}$ ).

Figure 4.4 3D-FDTD simulated electromagnetic field distributions of a gold i) nanosphere, ii) nanorod and iii) nanostar under excitations of (a,c,e) 532 $\mathrm{nm}$ and $(\mathrm{b}, \mathrm{d}, \mathrm{f}) 785 \mathrm{~nm}$ lasers. The intensity scale is normalized as $\mathrm{E} / \mathrm{E}_{0}$.

Figure 4.5 Schematical illustration of preparation of gold@MGITC@ $\mathrm{SiO}_{2}$ and its DNA functionalization. 
Figure 4.6 UV-visible absorption spectra of gold nanosphere, nanorod and nanostar@MGITC@SiO

Figure 4.7 TEM images of (a) gold nanosphere@MGITC@ $\mathrm{SiO}_{2}$, (b) gold 90 nanorod@MGITC@SiO 2 and (c)gold nanostar@MGITC@SiO

Figure 4.8 Raman spectra of gold nanosphere@MGITC@ $\mathrm{SiO}_{2}$ under (a) 532 nm and (b) 785 nm, gold nanorod@MGITC@ $\mathrm{SiO}_{2}$ under (c) 532 nm and (d) 785 nm lasers, and gold nanostar@MGITC@SiO $\mathrm{S}_{2}$ under (e) 532 nm and (f) $785 \mathrm{~nm}$ lasers.

Figure 4.9 (a) Color response and UV-visible aborption spectra of (b) gold nanospheres, and (c) gold nanosphere@MGITC@ $\mathrm{SiO}_{2}$ in representive solvents and salt concentrations.

Figure 4.10 Schematical assembly of a SERS device for monitoring of DNA hybridization based on gold nanostar@MGITC@SiO

Figure 4.11 SERS spectra of (a) $\mathrm{DNA}-\mathrm{SiO}_{2}$ slides, (b) uncomplementary gold nanostar@MGITC@ $\mathrm{SiO}_{2}$ with $\quad \mathrm{DNA}_{-} \mathrm{SiO}_{2}$ slides, and (c) complementary gold nanostar@MGITC@SiO $\mathrm{Si}_{2}$ with DNA-SiO ${ }_{2}$ slides.

Figure 5.1 Schematic illustrations of (a) preparation procedure of the SERS sensor based on gold nanostar@MGITC@ $\mathrm{SiO}_{2}$ as signaling probe and (b) operation principle of the SERS sensor for ATP detection. Inset in (b) shows the molecular structure of ATP.

Figure 5.2 Raman spectra of (a) gold nanostar@MGITC@ $\mathrm{SiO}_{2}$ in solution, (b) the SERS sensor with gold nanostar@MGITC@ $\mathrm{SiO}_{2}$ as signaling probe, and (c) the SERS sensor after exposure to $100 \mathrm{nM}$ ATP.

Figure 5.3 (a) SERS spectra of MGITC as a function of ATP concentration in the SERS sensor based on gold nanostar@MGITC@ $\mathrm{SiO}_{2}$ as signaling probe, (b) concentration-dependent response of the Raman intensity to the initial Raman intensity ratio at $1618 \mathrm{~cm}^{-1}$, and (c) the linear range in the low concentration region of (b).

Figure 5.4 SERS spectra of the SERS sensor based on the gold nanostar@MGITC@ $\mathrm{SiO}_{2}$ as signaling probe after exposure to solutions with various concentrations of (a) GTP and (b) CTP.

Figure 6.1 (a) Optical absorption spectra and (b) fluorescence emission spectra of representative $\mathrm{CdSe} / \mathrm{ZnS}$ core/shell quantum dots dispersed in $\mathrm{CH}_{2} \mathrm{Cl}_{2}$.

(c) Optical photos of representative $\mathrm{CdSe} / \mathrm{ZnS}$ core/shell quantum dots in $\mathrm{CH}_{2} \mathrm{Cl}_{2}$ under $365 \mathrm{~nm}$ excitation.

Figure 6.2 (a) TEM image (scale bar: $20 \mathrm{~nm}$ ) and (b) HRTEM image (scale bar: 2 $\mathrm{nm})$ of $\mathrm{CdSe} / \mathrm{ZnS}$ core/shell quantum dots with the fluorescence emission wavelength of $570 \mathrm{~nm}$.

Figure 6.3 XPS survey scan of $\mathrm{CdSe} / \mathrm{ZnS}$ core/shell quantum dots with the fluorescence emission wavelength of $570 \mathrm{~nm}$.

Figure 6.4 FTIR of $\mathrm{CdSe} / \mathrm{ZnS}$ core/shell quantum dots with the fluorescence emission wavelength of $570 \mathrm{~nm}$.

Figure 6.5 Water-soluble $\mathrm{CdSe} / \mathrm{ZnS}$ quantum dots through the ligand exchange method. Optical photo shows the excellent water solubility in PBS. 
Figure 6.6 XPS (a) survey scan, (b) C1s core level and (c) S2p core level of 3mercaptopropionic acid-modified $\mathrm{CdSe} / \mathrm{ZnS}$ quantum dots.

Figure 6.7 FTIR spectra of 3-mercaptopropionic acid-modified $\mathrm{CdSe} / \mathrm{ZnS}$ quantum dots.

Figure 6.8 Schematic illustration of the quantum dot (energy donor)-DNA-gold nanoparticle (acceptor) hybrid system in the presence of $\mathrm{Hg}$ (II) ions and used DNA sequences.

Figure 6.9 (a) Extinction spectra of $3 \mathrm{~nm}, 15 \mathrm{~nm}$ and $80 \mathrm{~nm}$ gold nanoparticles, and fluorescence emission spectra of DNA-functionalized quantum dots. (bd) TEM images of $3 \mathrm{~nm}, 15 \mathrm{~nm}$ and $80 \mathrm{~nm}$ gold nanoparticles, respectively.

Figure 6.10 Fluorescence spectra of the solution with (a) 10 base DNA-, (b) 20 base DNA- and (c) 30 base DNA-functionalized QDs without gold nanoparticles in the absence and presence of $100 \mathrm{nM} \mathrm{Hg}^{2+}$. The FL intensity shows no observable change after addition of $100 \mathrm{nM} \mathrm{Hg}{ }^{2+}$, indicating that $\mathrm{Hg}^{2+}$ makes negligible contribution to quenching of fluorescence from QDs in the present work.

Figure 6.11 Quenching of fluorescence emission of the quantum dots by the gold nanoparticles with different sizes (3 nm, $15 \mathrm{~nm}$ and $80 \mathrm{~nm}) ;(a)$ Fluorescence emission spectra of the solutions containing the DNAquantum dots and the DNA-gold nanoparticles after addition of various concentrations ( 0 to $60 \mathrm{nM}$ ) of $\mathrm{Hg}$ (II) ions; (b) Normalized fluorescence emission intensity as a function of $\mathrm{Hg}$ (II) ions; (c) Stern-Volmer plots showing the efficiencies of quenching by three types of gold nanoparticles with different sizes.

Figure 6.12 Fluorescence emission spectra of solutions of $\mathrm{CdSe} / \mathrm{ZnS}$ quantum dots and $3 \mathrm{~nm}$ gold nanoparticles when it uses DNA sequence of (a) 20 base pairs and (b) 30 base pairs, $15 \mathrm{~nm}$ gold nanoparticles when it uses DNA sequence of (c) 20 base pairs and (d) 30 base pairs, and $80 \mathrm{~nm}$ gold nanoparticles when it uses DNA sequence of (e) 20 base pairs and (f) 30 base pairs in the absence and presence of $60 \mathrm{nM} \mathrm{Hg}^{2+}$.

Figure 6.13 Experimental data points of the quenching efficiency and theoretical curves of the quenching efficiency versus the separation distance that were fitted based on the FRET and NSET models for (a) $3 \mathrm{~nm}$ gold nanoparticles, (b) $15 \mathrm{~nm}$ gold nanoparticles and (c) $80 \mathrm{~nm}$ gold nanoparticles.

Figure 7.1 (a) optical absorption and fluorescence emission spectra of TOPO$\mathrm{CdSe} / \mathrm{ZnS}$ quantum dots in $\mathrm{CH}_{2} \mathrm{Cl}_{2}$. Inset shows the optical photo of TOPO-CdSe/ZnS quantum dots in $\mathrm{CH}_{2} \mathrm{Cl}_{2}$ under excitation of $365 \mathrm{~nm}$ laser. (b) TEM image of TOPO-CdSe/ZnS quantum dots.

Figure 7.2 (a) Schematic illustration of the operating principle of the QD/DNA/gold nanoparticle ensemble sensor for $\mathrm{Hg}^{2+}$ detection. (b) Fluorescence emission spectra and (c) Photograph under $365 \mathrm{~nm}$ laser excitation of the QD/DNA/gold nanoparticle solution (96 nM QDs, 104 
$\mathrm{nM}$ gold nanoparticles and $0.1 \mathrm{mM}$ ethylenediamine in $0.3 \mathrm{M}$ PBS) before and after addition of $1 \mu \mathrm{M} \mathrm{Hg}^{2+}$.

Figure 7.3 Fluorescence emission spectra of DNA-CdSe/ZnS quantum dots in the absence and presence of $60 \mathrm{nM}$ and $100 \mathrm{nM}$.

Figure 7.4 Time-dependent fluorescence emission intensity at $572 \mathrm{~nm}$ of the QD/DNA/gold nanoparticle system at $1 \mu \mathrm{M} \mathrm{Hg}^{2+}$ concentration of (96 $\mathrm{nM} \mathrm{CdSe} / \mathrm{ZnS}$ QDs, $104 \mathrm{nM}$ gold nanoparticles and $0.1 \mathrm{mM}$ ethylenediamine in 0.3 M PBS).

Figure 7.5 (a) Fluorescence emission spectra of the QD/DNA/Au NP system in 0.3 M PBS solution after addition of various concentrations of $\mathrm{Hg}^{2+}(0,1,2$, $3,4,5,6,8,10,15,20,30,40,60,80,100,200,500$ and $1000 \mathrm{nM})$ into the $0.3 \mathrm{M}$ PBS solution (96 $\mathrm{nM}$ QDs, $104 \mathrm{nM}$ Au NPs and $0.1 \mathrm{mM}$ ethylenediamine). (b) Plot of fluorescence quenching efficiency as a function of the $\mathrm{Hg}^{2+}$ concentration. (c) linear region of (b).

Figure 7.6 Fluorescence quenching efficiency in the presence of various metal ions. The concentration of each metal ion is $100 \mathrm{nM}$ (96 nM QDs, 104 $\mathrm{nM} \mathrm{Au}$ NPs and $0.1 \mathrm{mM}$ ethylenediamine in $0.3 \mathrm{M}$ PBS).

Figure 7.7 (a) Fluorescence emission spectra of the QD/DNA/Au NP system in river water containing various concentrations of $\mathrm{Hg}^{2+}(0,1,2,4,6,8$, $10,15,20,30,40,60,80,100,200,500$ and $1000 \mathrm{nM})(96 \mathrm{nM}$ QDs, $104 \mathrm{nM} \mathrm{Au} \mathrm{NPs,} 0.1 \mathrm{mM}$ ethylenediamine and $0.3 \mathrm{M} \mathrm{NaCl}$ in the mixture of the PBS with the river water). (b) Plot of the fluorescence quenching efficiency as a function of the $\mathrm{Hg}^{2+}$ concentration in river water. (c) linear region of (b). 


\section{CHAPTER 1: INTRODUCTION}

\subsection{Motivation}

Rapid advances in technology and industrialization have improved and will continue to improve living qualities of human beings for a long time ago. Nevertheless, they also actually result in releases of lots of hazardous substances into our surrounding environments, thus exert potential environmental risks and toxicities to human health. In addition, food security issues have been emerging in both developed and developing countries in despite of long-term efforts. Illegal food additives from high-technology are added to daily food or drink by profit-driven manufacturers, and threaten a big community's health. Typically, in USA, the Defusco's salmonella outbreak has recently sickened 60 people in Rhode Island and Massachusetts [1]. Jennie-O Turkey burgers purchased from Sam's Club locations are being recalled after being linked to at least 12 Salmonella illnesses occurred from December 2010 to March 2011 in ten states of USA [2]. In 2008, the tainted milk produced by Sanlu, one of the biggest food security events in China, killed at least six children and made 300, 000 other people ill because of addition of melamine to raw milk to make it appear high in protein [3]. Also, scandals were found in Soccer players and track athletes who made use of illegal drugs to improve their performances. In addition, Industrial sources including coal and gold mining, solid waste incineration, wood pulping, fossil fuel combustion and chemical manufacturing release persistent and toxic heavy metals such as mercury, copper, arsenic, and so on, into the solid surface and 
surface waters. These substances that are either real or potential risks to health may enter the food supply and consequently cause illness by food poisoning.

The increasing demands for high-quality life require more efficient and simple approaches to ensure homeland security including forensic science, monitoring of controlled substances, food safety, and detection of counterfeiting, etc. [4]. Analytes to be monitored or quantified vary from illegal food additives, drug to viruses, bacteria, protein, DNA in a wide range of matrices from solid, surface waters to air. Currently, some approaches are routinely used to detect analytes in wide fields shown above. These approaches include electrochemical approach, atomic absorption spectroscopy, cold vapor atomic fluorescence spectrometry, gas chromatography, mass spectrometry, super-resolution microscopy, and colorimetric approach, and so on. Also, there are numerous companies involved in developing commercial available devices for homeland security such as Tetracore, ICx Technologies and Smiths Detection. However, many of these approaches require complicated, multistep sample preparation and sophisticated instrumentation as well as give the drawback of poor sensitivity. There is a strong incentive to develop reliable, convenient, efficient and cost-effective approaches for the monitoring of food safety, water and air quality, and chemical warfare agents along with biological and nuclear weapons.

Although the specific requirements may vary according to different applications, a reliable sensor commonly requires (1) high sensitivity, (2) high selectivity, (3) real-time and point-ofcare (or on-site) detection, (4) continuous detection, (5) multiplexing detection, (6) low-cost, and (7) ease operation and portability, and so on. Among a large number of sensing strategies, optical assays particularly provides an optimized combination of speed, sensitivity, robustness and 
safety. Therefore, in the present work, we aim to develop sensing strategies with high sensitivity, high selectivity and potential portability.

Both surface-enhanced Raman spectroscopy (SERS) [7] and fluorescence spectroscopy have been demonstrated to be both of promising sensing strategies in a wide range of applications. SERS has potential advantages in the application of chemical sensing include [5] (1) the fingerprint information of targets of interest, (2) the enabled detection of different species at the same time, (3) real-time, portable point-of-care detection capability as well as (4) ease operation, etc. Due to these unique attributes, SERS has been potentially considered to be a promising portable point-of-care detection among all measurement techniques. However, SERS sensor still is in the infancy of academic study, and no reliable and low-cost commercial device is available. Also, the physical and chemical essence behind the SERS remains unclear, and development of high-quality SERS sensor is being desired. It is widely accepted that the SERS enhancement is attributed to the strong electromagnetic enhancement (EM) and the chemical enhancement (CE), and entanglement of two enhancement mechanisms allows the difficult investigation of their cross-effects. In addition, physical essence of effects of substrates with distinct LSPR on SERS enhancement still needs to be further clarified.

On the other hand, fluorescence resonant energy transfer (FRET) is extensively used to design the fluorescent senor. Typically, most of fluorescent sensors employ organic dyes as energy donors in resonance energy transfer (RET)-based sensors, which suffer drawbacks of narrow excitation profile, nonsymmetrical emission spectra and photobleaching, etc. Semiconductor nanocrystals (quantum dots, QDs) have emerged as novel fluorescent labels and are substituting for the conventional organic fluorophores [6]. This is ascribed to the great advantages of inorganic nanocrystals over the conventional organic dyes in aspects of optical and 
electronic properties. Semiconducting QDs exhibit broad excitation profiles, narrow and symmetric emission spectra, high photostability, high quantum efficiency and potential multiplexing detection capability. In addition, Strouse et al. reported that use of $1.5 \mathrm{~nm}$ gold nanoparticles as energy acceptors enables more efficient energy transfer and bigger detection length, leading to the nanometal surface energy transfer (NSET) mechanism [8]. Despite that successive efforts have been made from experimental and theoretical points of view, most of their work was focused on small gold nanoparticles with little or no localized surface plasmon resonance (LSPR) absorption in the NSET system, and involvement of LSPR and its effect on the energy transfer have been paid less attention.

To sum up, this project is motivated by demands (1) to develop detection tools with high reliability, high sensitivity and specificity, and portability, etc., (2) to fundamentally understand functional components in SERS and RET-based sensors and (3) to investigate contributions of LSPR to the improved performances in SERS- and RET-based sensors.

\subsection{Objectives}

The overall objective of the project is to investigate the energy transfer and the charge transfer processes in the semiconductor-metal nanoparticle systems that may be correlated with LSPR and to subsequently develop effective sensing platforms based on SERS and energy transfer. In order to realize the overall objective mentioned above, the following specific aims will be pursued.

\section{(1) To investigate the charge transfer-induced electromagnetic enhancement in SERS}


Both EM and CE mechanisms are widely accepted to make main contributions to SERS. We modify gold nanoparticles of LSPR absorption around $520 \mathrm{~nm}$ using different molecular structures, which are 3-mercaptopropanol acid (MPA), p-mercaptobenzoic acid (MBA), thiophenol (TP) and L-cysteine (Cys), respectively. Both MBA and TP possess similar planar structures while there are linear structures for both MPA and Cys. In most of systems, crosseffect of electromagnetic resonance and charge transfer is entangled. MBA, MPA and Cys contain $-\mathrm{COOH}$ group, which can interact with the divalent ions through coordinate chemistry. We use divalent ions to link two gold nanoparticles together, and then create "hot spots" in which SERS of molecules is significantly amplified. The average SERS spectra are collected in these systems, and the SERS activity is investigated. The contributions of both charge transfer and LSPR to the EM enhancement will be discussed.

(2) To investigate size and shape effects on SERS enhancement, and theoretically correlate

\section{LSPR with experimental SERS enhancement factor}

Previous experimental and theoretical efforts have been made to understand the size, shape and composition-dependent LSPR properties in gold and silver substrates. Substrates with different sizes and shapes exhibit distinct LSPR position and electromagnetic field distribution. For example, concentration of electromagnetic field was found in the anisotropic nanostructures, especially sharp tips in triangles, elongated nanorods, nanowires or nanostars. Undoubtedly, these structures will possess distinct SERS enhancement based on the $|E|^{4}$-approximation dependence in SERS. We will focus on the SERS in different sized gold nanospheres, gold nanorods and gold nanostars. Three-dimensional finite-difference time domain (FDTD) analysis 
will be carried out to understand the distinct SERS enhancements in these different shaped gold structures.

\section{(3) To develop SERS sensors for monitoring DNA hybridization and ATP detection}

Based on the above investigations, we will prepare a Raman probe for biocompatible applications. The gold@malachite green isothiocyanate(MGITC)@ $\mathrm{SiO}_{2}$ sandwiched structure will be prepared because the $\mathrm{SiO}_{2}$ protective layer allows easy surface functionalization and stability of MGITC molecules within the $\mathrm{SiO}_{2}$ layer. This kind of sandwiched structure will be used for monitoring DNA hybridization. Also, a SERS sensor for adenosine triphosphate (ATP) detection will be developed with DNA aptamer as the recognition element.

(4) To investigate energy transfer from QDs to gold nanoparticles with and without LSPR absorption

Previous work was focused on the small gold nanoparticles with little or no LSPR absorption in which NSET mechanism was proposed. We will investigate the energy transfer between QDs and gold nanoparticles with different sizes, and gain some mechanistic insights into the energy transfer when LSPR is involved.

\section{(5) To develop fluorescent sensors for $\mathrm{Hg}^{2+}$ detection based on QD/DNA/gold ensembles}

After the mechanism of the interaction of QDs with gold nanoparticle is clarified, a fluorescent sensor will be developed for $\mathrm{Hg}^{2+}$ detection using QDs as energy donors and gold nanoparticles as energy acceptors. The complementary oligonucleotides are designed and synthesized with given $\mathrm{T}-\mathrm{T}$ mismatches. The specific binding of $\mathrm{T}-\mathrm{Hg}^{2+}-\mathrm{T}$ renders the sensor 
excellent selectivity. We check the sensitivity, and selectivity as well as the anti-interference capability, etc. The $\mathrm{Hg}^{2+}$ detection in river water matrix from the Monongahela River is carried out to know about the real use in the practical application.

\subsection{Significance}

In general, the enhancement factor of Raman signal is dependent on the Raman reporter and the substrate. Furthermore, SERS essentially originates from the LSPR, so developing the SERS substrate with the strong and controllable LSPR absorption will improve the performances of SERS sensor. SERS sensor is in its infancy even considered to be the most promising detection technique in the point-of-care application. Investigation of various components of SERS sensor will benefit to the understanding of functionality of each component and the construction of SERS sensor. On the other hand, the incorporation of QDs as fluorophores into the fluorescent sensor is expected to take advantages over the conventional fluorescent sensor based on the organic dyes in stability, cost-consumption, and multiplexing capability, etc. Use of gold nanoparticles with strong LSPR absorption will benefit to the improvement of performances of sensors.

We believe that use of gold nanoparticles in SERS and fluorescent sensors is a wise strategy to improve the performances of sensors and will benefit to high sensitivity, high stability as well as low-cost, and is of significance from fundamental and applicable points of view. More importantly, the developed sensors provide possibility for multiplexing detection and on-site detection in an ease operation and low-cost manner.

Incorporation of nanomaterials into energy transfer and SERS sensors will benefit to the sensor in the following aspects: (1) improved detection limit, (2) reduced dimension of sensor, 
(3) reduced energy consumption, and (4) enhanced multiplexing detection capability in a small size, etc. Consequently, use of nanomaterials will lead to the improvement of performances of SERS sensor and fluorescent sensor in aspects mentioned above.

\subsection{Dissertation organization}

In this dissertation, we focus on gold nanoparticles in constructions of SERS and fluorescent sensors, and improvement of performances in these sensors because of involvement of different LSPR features. In addition to experimental investigations, theoretical analysis is carried out to gain some mechanistic insights into physical essences in sensors containing various kinds of gold nanoparticles. This dissertation is divided into eight chapters.

- Chapter 1 overviews the research background and motivation, and states the objective and the significance of the project.

- Chapter 2 provides the background knowledge and recent developments in SERS and RETbased fluorescent sensors, along with introduction of LSPR in both kinds of sensors, which are necessary to understand the experiments, discussion and conclusions in the following chapters.

- Chapter 3 performs the fundamental investigation of chemical enhancement and electromagnetic enhancement in SERS, and mainly focuses on the chemical contribution to the electromagnetic enhancement in SERS.

- Chapter 4 deals with the shape effect in SERS. Gold nanospheres, nanorods and nanostars are used, and correlation of experimental results is carried out with the results from threedimension FDTD simulation. In addition, Raman tagged gold nanostructures encapsulated by 
$\mathrm{SiO}_{2}$ are investigated for biological applications and its application in DNA hybridization monitoring is demonstrated.

- Chapter 5 is engaged in the development of SERS sensor for ATP detection based on the gold nanostar@MGITC@ $\mathrm{SiO}_{2}$ as the signaling probe.

- Chapter 6 is devoted to the energy transfer from QDs to gold nanoparticles. Involvement of LSPR into the energy transfer is addressed and its energy transfer mechanism is analyzed.

- Chapter 7 describes a real fluorescent sensor for $\mathrm{Hg}^{2+}$ detection based on the energy transfer between QDs and gold nanoparticles. Sensitivity, selectivity and anti-interference capability of this sensor are examined in both buffer solution and river water matrix from the Monongahela River.

- Chapter 8 gives a summary of the whole work in this dissertation, itemizes the conclusions, and discusses future directions that do not fall into the scope of this work.

\section{References}

[1] Massachusetts Mom Sues Rhode Island Bakery over Tainted Pastries. http://www.insurancejournal.com/news/east/2011/04/06/193439.htm. (accessed on June 15, 2011).

[2] Jennie-O Recalls Turkey Burgers Sold at Sam's Club: Salmonella in 10 States. http://www.walletpop.com/2011/04/02/jennie-o-recalls-turkey-burgers-sold-at-sams-clubsalmonella-i/. (accessed on June 15, 2011). 
[3] 2 face execution over China poison milk scandal. http://www.msnbc.msn.com/id/28787126/ns/world_news-asia_pacific/t/face-execution-overchina-poison-milk-scandal/. (accessed on June 15, 2011).

[4] Sekhar, P. K.; Brosha, E. L.; Mukundan, R.; Garzon, F. H. Electrochem. Soc. Interf., 2010, $35-40$

[5] Golightly, R. S.; Doering, W. E.; Natan, M. J. ACS Nano, 2009, 3, 2859-2869.

[6] Medintz, I. L.; Clapp, A. R.; Mattoussi, H.; Goldman, E. R.; Fisher, B.; Mauro, J. M. Nat. Mater., 2003, 2, 630-638.

[7] Fleischmann, M.; Hendra, P. J.; McQuillan, A. J. Chem. Phys. Lett., 1974, 26, 163-166.

[8] Jennings, T. L.; Singh, M. P.; Strouse, G. F. J. Am. Chem. Soc., 2006, 128, 5462-5467. 


\section{CHAPTER 2: BACKGROUND AND LITERATURE OVERVIEW}

\subsection{Surface plasmon resonance absorption}

\subsubsection{Fundamentals of surface plasmon resonance}

Surface plasmon resonance (SPR) arises from the collective charge oscillations of the free conduction electrons of the metal in resonance with incident electromagnetic radiation [1,2]. For many metals such as $\mathrm{Pb}, \mathrm{In}, \mathrm{Hg}, \mathrm{Sn}$ and $\mathrm{Cd}$, the plasma frequency lies in the UV part of the spectrum, there is no color effect observed, and more importantly, they are unstable in ambient condition while conversely, $\mathrm{Cu}, \mathrm{Ag}$ and $\mathrm{Au}$ show plasmon frequency in the visible-light region [3]. The formation of a surface plasmon can be briefly seen as follows: the electric field of the incoming radiation induces the formation of a dipole in the nanoparticle, and there is a restoring force that tries to compensate it, so that a unique resonance frequency matches this electron oscillation within the nanoparticle (Figure 2.1). In nanoscale objects, the size becomes comparable with or smaller than the wavelength so that the electromagnetic oscillation modes then exist in the discrete form. In this case, SPR is also called localized surface plasmon resonance (LSPR), which plays dominant roles in SERS and other LSPR-related optical effects. For elongated nanoparticles, two different resonance modes are observed as a function of their orientation with respect to the direction of the electric field of incoming electromagnetic radiation. The resonance parallel to the long axis of the structures refers to the longitudinal surface plasmon resonance band while the resonance perpendicular to the long axis leads to a transverse surface plasmon resonance band (Figure 2.1b) [3]. 
(a)
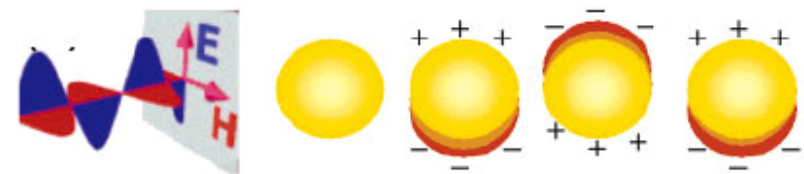

(b)

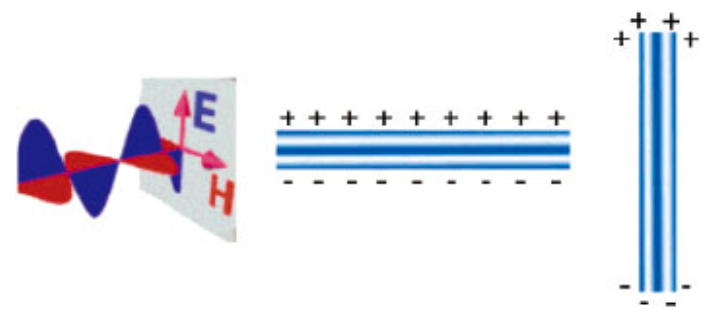

Figure 2.1 Schematic illustration of (a) an electromagnetic radiation with a metal nanosphere. A dipole is induced, which oscillates in phase with the electric field of the incoming light. (b) Transverse and longitudinal oscillations of electrons in a metal nanorod [3].

Over the past several decades, theoretical efforts advance in modeling the mater-light interaction [1,4-7]. The classical work in light absorption and scattering was carried out by Mie in 1908, which presented a solution to Maxwell's equation and then described the extinction spectra (including absorption and scattering) of spherical particles. According to the Mie theory, one can predict the position and shape of plasmon absorption spectrum for spherical and spheroidal metal particles, shown by the following equation:

$$
E(\lambda)=\frac{24 \pi^{2} N R^{3} \varepsilon_{M}{ }^{3 / 2}}{\lambda \ln (10)}\left[\frac{\varepsilon_{i}(\lambda)}{\left(\varepsilon_{r}(\lambda)+\chi \varepsilon_{M}(\lambda)\right)^{2}+\varepsilon_{i}(\lambda)^{2}}\right]
$$

where $\varepsilon_{r}$ and $\varepsilon_{i}$ are the real and imaginary components of the dielectric function of the metal, $\varepsilon_{M}$ is the dielectric function of the surrounding medium, $R$ is the radius of the particle, $\chi$ is the factor related to the eccentricity of the particle and $N$ is the number of atoms in the particle. Furthermore, the subsequent derivatives of Mie's work have given further understanding and 
predictions for optical absorption of elongated structures and other nonspherical structures. The most commonly used one is from Garnett in which the optical properties can be described by the dielectric function of effective medium $\left(\varepsilon_{\text {eff }}\right)$ as follows $[8,9]$ :

$$
\frac{\varepsilon_{\text {eff }}-\varepsilon_{\mathrm{M}}}{L \bullet \varepsilon_{\text {eff }}+(1-L) \bullet \varepsilon_{\mathrm{M}}}=f \bullet \frac{\varepsilon_{r}(\lambda)-\varepsilon_{\mathrm{M}}}{L \bullet \varepsilon_{r}(\lambda)+(1-L) \bullet \varepsilon_{\mathrm{M}}}
$$

where $\varepsilon_{\mathrm{r}}$ is the real part of the dielectric function of the metal particle, $\varepsilon_{\mathrm{m}}$ the dielectric function of the surrounding medium, $L$ the shape factor of the particle, and $f$ the filling factor reflecting the extent of the particle-particle interactions by means of their dipolar fields.

\subsubsection{Specific factors influencing LSPR}

Based on Mie's theory, the features of the LSPR absorption around the nanoparticles strongly depend on particle size, geometrical shape, composition of materials, medium refractive index and interparticle interactions, etc. The following descriptions are devoted to introducing these factors involved in LSPR.

Liz-Marzán et al. systematically investigated the size-dependence of LSPR absorption in gold nanospheres from theoretical and experimental points of view [3]. The LSPR absorption redshifts with the increasing particle size although the increasing extent is much smaller than that in the geometrical shape. Nevertheless, the effect of particle size is difficult to be investigated in real environment because in general, most of particles exist in colloidal status, and the interparticle interaction still affects the LSPR in both intensity and position. Recently, Van Duyne et al. employed a correlated high resolution transmission electron microscopy (HRTEM) LSPR technique to monitor the size-dependence in silver bipyramids, silver cubes, and gold cubes, in which statistical analysis was performed on a large number of single particles [10]. It was found that the slope of the dependence of LSPR peak on size for silver bipyramids increases 
as the edges become sharper. At the same time, an increase in substrate refractive index, and a change of composition from silver to gold all lead to a red-shift of the LSPR frequency. Investigations have suggested that the red-shift in LSPR peak with particle size and concentration originates from the size-dependent dielectric function of gold core electrons [9].

The development of synthetic techniques, largely deriving from colloid chemistry as well as lithographic techniques, has allowed researchers to control the geometry of nanomaterials [1115]. Various shaped particles (nanospheres, nanorods, and nanowires, etc.) and assemblies on solid substrates have been demonstrated to generate distinct LSPR absorption in both position and distribution $[3,11,16,17]$. Concentration of electromagnetic field has been found in the anisotropic nanostructures, especially sharp tips in triangles, elongated rods and nanostars, etc. Theoretical calculation and experiment results indicate that the transverse mode blue-shifts while the longitudinal mode red-shifts with the increasing aspect ratio (length to width ratio) of gold nanorods. More importantly, a small change in aspect ratio can adjust the longitudinal plasmon across the whole visible region toward the near infrared region, which is significantly effective in comparison with that in size change. Typically, the seed-mediated growth method is used to make gold nanorods with various aspect ratios [11,12]. EI-Sayed, Murphy and Xia groups have made great contributions to the synthetic methodology of gold nanorods and the evolution of optical properties in these gold nanorods. EI-Sayed et al. successfully synthesized gold nanorods with aspect ratios of 1 to 10 , which display a longitudinal plasmon mode from 600 to $1300 \mathrm{~nm}$ [18]. Xia et al. developed an approach to make gold nanowires using wetting chemistry and other shaped gold nanomaterials for SERS application [19]. Various shaped patterns on solid substrates have been prepared by well-developed lithographic techniques, which enable us to probe the shape-dependent LSPR and the coupling of LSPR. As well-known, the coupling 
interactions between patterns depend on not only the shape and composition of materials but also the spacing and size. The coupling between patterns with different shapes and spacing can generate new surface plasmon characteristics [4, 20-23].

In addition, Mie theory and its derivatives show that dielectric functions of metals and its surrounding medium produce effects on the LSPR position and intensity, which renders the possibility of SPR sensors in principle [24-29]. Addition of analytes causes the shift of LSPR absorption due to the change in the dielectric function. In this case, Otto and Kretschmann configurations are two of the most commonly used set-ups for chemical sensing [30,31].

\subsection{Surface-enhanced Raman scattering and fluorescence}

\subsubsection{Surface-enhanced Raman scattering}

As described previously, SERS is a powerful technique for chemical sensing, biological imaging and environmental monitoring, etc. In principle, SERS originates from the amplified electromagnetic field (or LSPR) around the nanostructures. When an incident light is directed to the metal nanostructure, the induced LSPR occurs at the surface and then generates the amplified electromagnetic field. As a result, the modified electromagnetic fields inside $\left(E_{\text {in }}\right)$ and outside $\left(E_{\text {out }}\right)$ of the sphere vary, which can be calculated by the Maxwell's equations $[1,6]$.

$$
\begin{gathered}
E_{\text {in }}=\frac{3 \varepsilon_{M}}{\varepsilon(\lambda)+2 \varepsilon_{M}} E_{0} \\
\boldsymbol{E}_{\text {out }}=E_{0} z-\alpha E_{0}\left[\frac{z}{r^{3}}-\frac{3 z}{r^{5}}(z \mathbf{z}+x \boldsymbol{x}+y \boldsymbol{y})\right] \\
\alpha=g(\lambda) \alpha^{3} \quad \text { with } g(\omega)=\frac{\varepsilon_{\mathrm{r}}(\lambda)-\varepsilon_{\mathrm{i}}(\lambda)}{\varepsilon_{\mathrm{r}}(\lambda)+2 \varepsilon_{\mathrm{i}}(\lambda)}
\end{gathered}
$$


where $\varepsilon_{M}$ and $\varepsilon$ are the dielectric function of the medium and the metallic particles, $E_{0}$ is the magnitude of the incident field, $\alpha$ is the metal polarizability, $r$ is the radial distance, $x, y$, and $z$ are the Cartesian coordinates, $\boldsymbol{x}, \boldsymbol{y}$, and $\mathbf{z}$ are the unit vectors.

Figure 2.2 schematically illustrates operation principle of Raman spectroscopy in comparison with infrared spectra and Rayleigh scattering. The electric field of incident light distorts the molecule's electron cloud, causing it to undergo electronic transition to a higher energy 'virtual state' (not a true quantum mechanical state of the molecule). Raman scattering results in the release of a scattered photon with different energies from the incident photon, and the difference in energy being equal to the vibrational transition is characteristic of the molecular structure, which is reflected with the form of peaks in the Raman spectra.

Due to the LSPR under the incident light, the electromagnetic wave around nanostructures is modified (eqs. 2.3 and 2.4) and consequently there is a huge amplification of electromagnetic field, which makes main contribution to the amplification of Raman scattering event. In addition, the electron transfer processes from metal to molecule or vice versa is also demonstrated to produce the SERS process, which will be introduced in detail later. Simply saying, factors influencing the SERS enhancement include the incident laser, detection set-up, SERS substrate and the analyte, etc. 


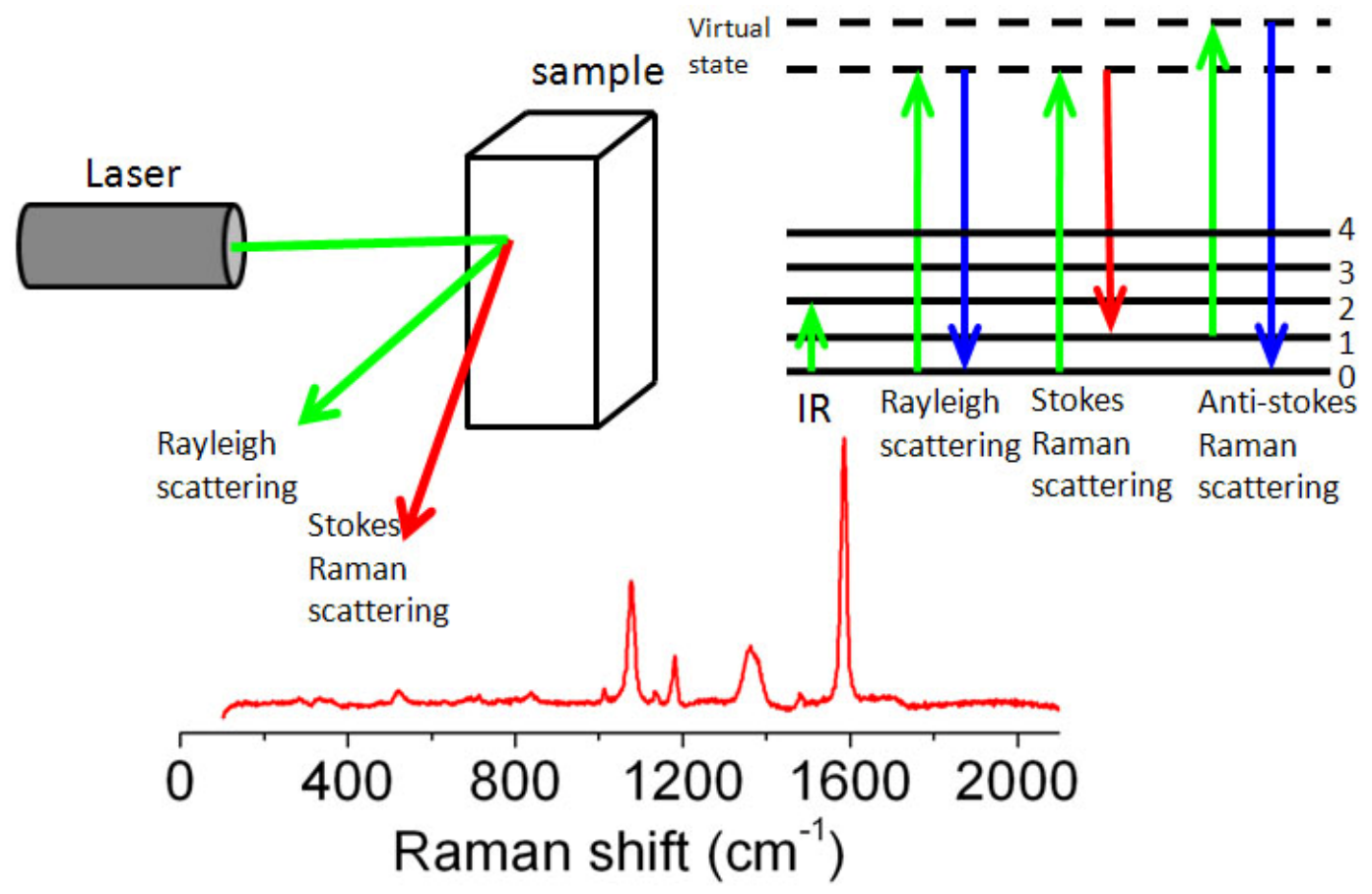

Figure 2.2 Schematic operation principle of Raman spectroscopy in comparison with infrared spectroscopy and Rayleigh scattering.

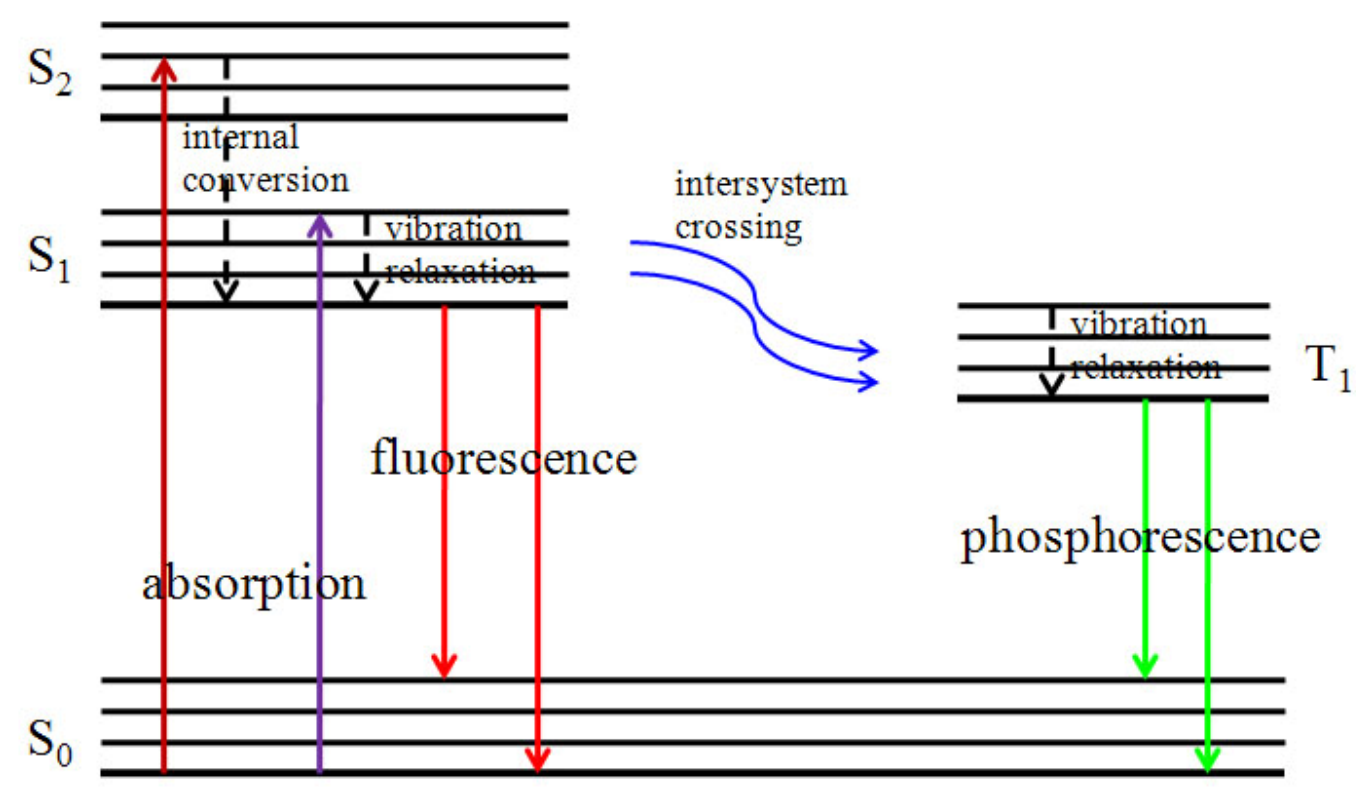

Figure 2.3 Jablonski diagram illustrating the excitation and emission process of a molecule. 


\subsubsection{Fluorescence and resonance energy transfer}

Photoluminescence is the emission of light from any substance and occurs from electronically excited states to the ground state accompanying with release of photons, which is typically divided into two categories: fluorescence and phosphorescence. The photoluminescence process of molecules can be usually illustrated by the Jablonski diagram (Figure 2.3). Fluorescence is emission of light from singlet excited states to the ground state, in which the spin orientation of electrons is kept constant, while phosphorescence comes from the inactivation of triplet excited states. Due to the nature of forbidden transition, phosphorescence emission rate is much slower than that of fluorescence. The fluorescence emission as well as the absorption is usually characteristic of the molecular structure.

As seen in Figures 2.3 and 2.4, the energy of emission is typically much less than that of absorption. The reasons are speculated to be due to the internal conversion from higher excited states to lower excited states, vibrational relaxation and other energy losses. The difference in energy between the absorbed photon and the emitted photon is called as Stokes shift. It should be noted that the mirror-image rule is followed between absorption and emission spectra, especially some of organic dyes, with some exceptions in semiconductor fluorescent materials (Figure 2.4).

The quantum yield $(\Phi)$ is an important character of a fluorophore, which describes the number of emitted photons relative to the number of absorbed photons. It is usually given by the following expression [33]:

$$
\Phi=\frac{\Gamma}{\Gamma+k_{n r}}
$$

where $\Gamma$ and $k_{\mathrm{nr}}$ are the emissive rate and non-radiative decay rate of the fluorophore, 


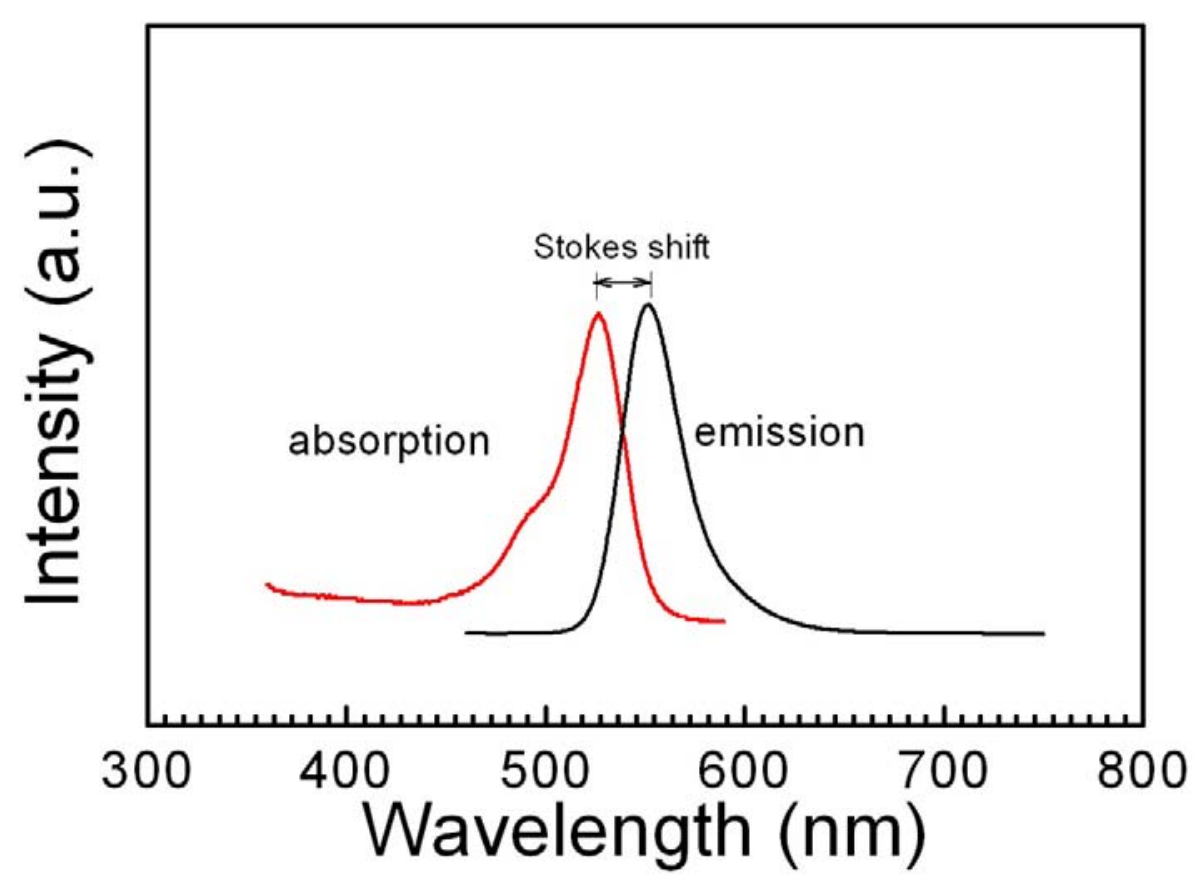

Figure 2.4 Absorption and fluorescence spectra of Rhodamine $6 \mathrm{G}$ in ethanol.

respectively. In practical applications, determination of the quantum yield is generally accomplished by comparison of the wavelength integrated intensity of the unknown to that of the standard [33]:

$$
\Phi=\Phi_{R} \frac{I}{I_{R}} \frac{O D_{R}}{O D} \frac{n^{2}}{n_{R}^{2}}
$$

where $I, O D$ and $n$ are the integrated intensity, the optical density and the refractive index of the unknown sample and reference sample $(R)$, respectively. In this case, both unknown and reference samples should hold the same excitation wavelength, otherwise the intensities need to be corrected.

Fluorescent sensors have been designed and used for applications in life-science field since 60 years ago. Resoance energy transfer (RET) is considered as a kind of efficient strategy to 
construct fluorescent sensors. RET typically occurs between an energy donor molecule in the excited state and an acceptor in the ground state. The donor molecules emit at short wavelengths that overlap with the absorption spectrum of the acceptor, and the acceptor does not necessarily fluoresce. The RET process does not undergo emission of photon from the donor and reabsorption by acceptor but is the result of long range dipole-dipole interactions between the donor and the acceptor. The energy transfer efficiency strongly depends on the spectral overlap between the fluorescence emission spectrum and the absorption spectrum. The conventional approach uses organic dyes as the acceptor, and in this process, the Förster resonance energy transfer (FRET) mechanism was proposed [34-36]. Recently, it has been found that replacement of organic dyes with small gold nanoparticles results in the nanometal surface energy transfer (NSET) and offers more efficient energy transfer at long separation distance [37-42]. Both FRET and NSET belongs to the RET process, and originates from the dipole-dipole interaction in essence. But more dipoles exist on the gold surface, and thus more dipoles can interact with the fluorophore in the gold system.

The energy transfer efficiency $(E)$ in RET process follows [43]:

$$
E=\frac{1}{1+\left(r / R_{0}\right)^{n}}
$$

where $r$ is the separation distance; $n=6$ for FRET and $n=4$ for NSET; $R_{0}$ is the separation distance at energy transfer efficiency of 50\%:

$$
\begin{gathered}
R_{0(\mathrm{FRET})}=0.211\left(\kappa^{2} \Phi_{\mathrm{D}}(n)^{-4} J(\lambda)\right)^{1 / 6} \\
R_{0(\mathrm{NSET})}=\left(0.225 \bullet \frac{\Phi_{\mathrm{D}}}{\omega_{\mathrm{D}}^{2}} \bullet \frac{1}{\omega_{F} k_{\mathrm{F}}} \bullet c^{3}\right)^{1 / 4}
\end{gathered}
$$


where $\Phi_{\mathrm{D}}$ is the quantum yield of the donor in the absence of acceptor, $n$ is the refractive index of the medium, $\kappa^{2}$ describes the relative orientation in space of the transition dipoles of the donor and the acceptor, $\omega_{\mathrm{D}}$ and $c$ represent the angular frequency of the donor emission and the light velocity in vacuum, $\omega_{\mathrm{F}}$ and $k_{\mathrm{F}}$ are the bulk gold angular frequency and Femi vector, respectively, and $J(\lambda)$ is the overlap integral between the normalized donor emission and the acceptor extinction coefficient.

The FRET efficiency can be experimentally measured by the integrated fluorescence intensity or life-time of the donor in the absence $\left(I_{D}, \tau_{D}\right)$ and presence $\left(I_{D A}, \tau_{D A}\right)$ of acceptor:

$$
E=1-\frac{I_{D A}}{I_{D}}=1-\frac{\tau_{D A}}{\tau_{D}}
$$

\subsection{Localized surface plasmon resonance in SERS and RET}

\subsubsection{Enhancement mechanisms in SERS}

Currently, it is widely accepted that the SERS enhancement is mainly attributed to electromagnetic enhancement (EM) and chemical enhancement (CE) mechanisms. It is found that the EM mechanism makes dominant contribution in SERS through comparing the enhancement factors of EM and CE [44-47]. The enhancement factors of EM and CE mechanisms are typically in the range of $10^{4}-10^{15}$ and $10-10^{2}$, respectively. Understanding physical essence of both EM and CE in SERS is paramount to manipulate and construct SERS sensors. We overview EM and CE enhancement mechanisms that contribute to the SERS effect in the following.

(1) Electromagnetic enhancement 
The EM enhancement mechanism is mainly due to the resonance excitation of plasmon oscillations in the metal nanoparticles by the incident laser light. The resonance excitation of LSPR results in an amplified electric field in the vicinity of the metallic surface. Figure 2.5 describes the generation of LSPR, and the consequent amplification of Raman scattering of analytes positioning at the "hot spots". The electromagnetic field enhancement due to the LSPR excitation under external incident light includes the local field enhancement at the molecule position and the radiation enhancement due to the modified Raman dipole [2]. Therefore, the SERS EM problem mainly lies in understanding the following two effects: modification of the EM field and the radiation properties of a dipole.

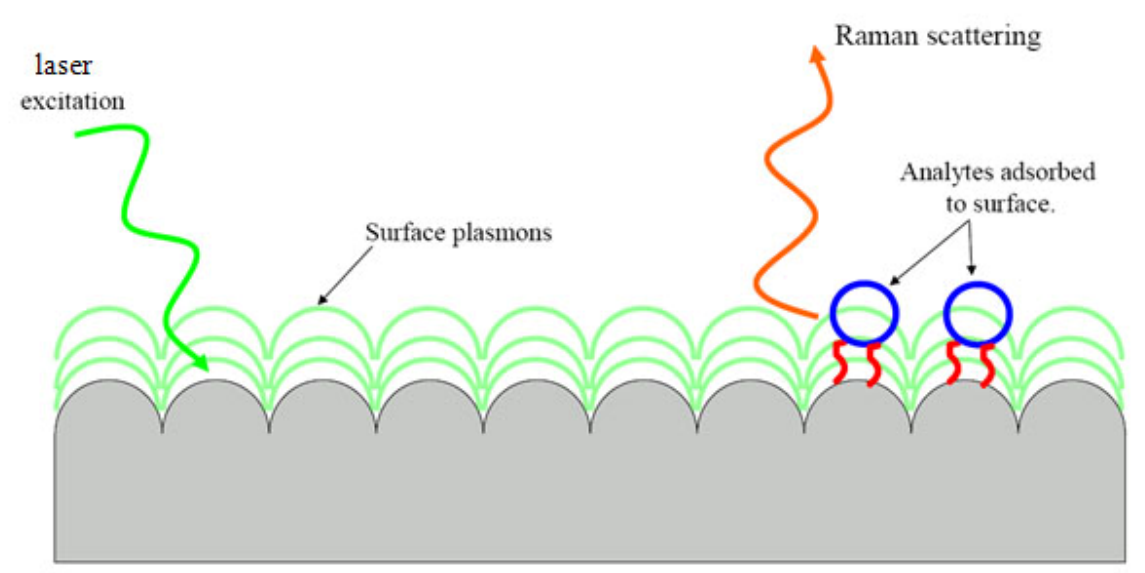

Figure 2.5 Schematic illustration of electromagnetic enhancement in surface-enhanced Raman scattering of a molecule on a gold or silver film on nanosphere.

The local electromagnetic field $\mathbf{E}_{\text {Loc }}$ at the molecule is very different, both in magnitude and orientation, to the incident field $\mathbf{E}_{\text {Inc }}$. The local field intensity enhancement factor can be given by: 


$$
M_{L o c}\left(\omega_{L}\right)=\frac{\left|\mathbf{E}_{L o c}\left(\omega_{L}\right)\right|^{2}}{\left|\mathbf{E}_{I n c}\right|^{2}}
$$

The local field intensity enhancement exists at the position close to the metallic surface, and is associated with a coupling to LSPR. As a result, the local field intensity enhancement factor is a function of the incident wavelength. Typically, the gap ("hot spots") between two metallic objects has the highest local field intensity enhancement.

In addition to the local field enhancement, the radiation enhancement factor is related to the modified electromagnetic field [2,4]. Nevertheless, this kind of enhancement is also dependent on the Raman scattering process. Therefore, simply assuming that the radiation enhancement is equivalent to the local field enhancement, the total SERS EM enhancement factor is a result combining with the local field enhancement and radiation enhancement:

$$
\begin{aligned}
E F & \approx M_{L o c}\left(\omega_{L}\right) M_{\text {Rad }}^{d}\left(\omega_{R}\right) \\
& \approx \frac{\left|\mathbf{E}_{L o c}\left(\omega_{L}\right)\right|^{2}}{\left|\mathbf{E}_{I n c}\right|^{2}} \frac{\left|\mathbf{E}_{L o c}\left(\omega_{R}\right)\right|^{2}}{\left|\mathbf{E}_{I n c}\right|^{2}} \\
& \approx \frac{\left|\mathbf{E}_{L o c}\left(\omega_{L}\right)\right|^{4}}{\left|\mathbf{E}_{I n c}\right|^{4}}
\end{aligned}
$$

This indicates that the total SERS EM enhancement factor follows a $|E|^{4}$ - approximation on the relative electromagnetic field intensity enhancement. This expression provides the theoretic fundaments for electromagnetic study of SERS.

(2) Chemical enhancement

In addition to the electromagnetic field enhancement from the LSPR coupling, SERS can be also chemically enhanced due to the charge transfer between the metallic particle and the 
adsorbed molecule, and the modulation of the metallic particle polarizability by the vibrational motion of the adsorbed molecule [44,48-50]. In the case of charge transfer mechanism, the transfer of an electron from the metal to a molecular orbit of the adsorbate will change the potential in the molecule and induce a change in position of the nuclei of the atoms. When the electron tunnels back to the metal, the molecule will return to the ground state. The charge transfer process facilitates the excitation of Raman scattering (Figure 2.6).

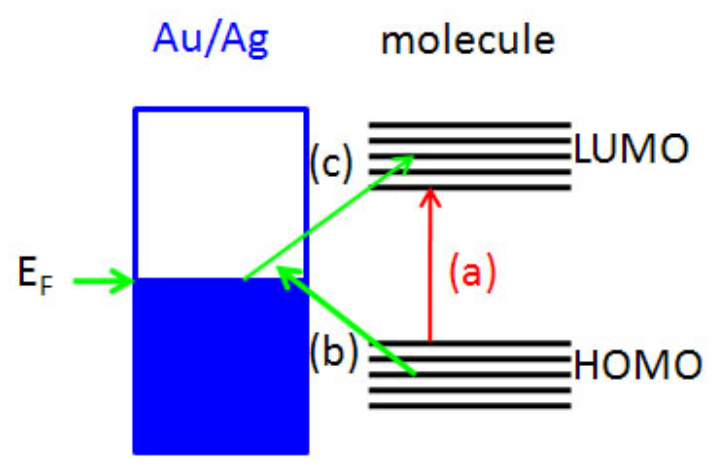

Figure 2.6 Schematic illustration of the charge transfer mechanism in a SERS process. (a) The laser energy directly excites the SERS process in resonance with an electronic transition of metal-molecules complex, and the metal disturbs the electronic structure of molecule and thus changes the polarizability; $(b, c)$ the electron transfer indirectly occurs through the metal.

As well-known, Raman scatterings of all molecules in the vicinity of SERS substrates are simultaneously amplified without any selectivity in the EM enhancement. However, previous results have indicated that the SERS process shows selectivity toward different molecules in some cases, which is attributed to the charge transfer mechanism. Different structural molecules 
exhibit distinct SERS activity even on the same SERS substrate. In addition, it is necessary to chemically bind Raman molecules onto the SERS substrate and form the metal-molecule complex. Thus, the charge transfer mechanism explains some phenomena which cannot be explained by the EM mechanism.

Both EM and CE mechanisms co-exist in most of systems, which makes difficult the mechanistic investigation in the practical work [50-52].

\subsubsection{Effect of LSPR on RET}

As mentioned above, RET occurs through the dipole-dipole interaction between the donor and the acceptor. Similar to LSPR in SERS, the enhanced electromagnetic field originating from the excitation of LSPR can influence radiative and nonradiative rates of a fluorophore due to a change in the optical local density of states [53-56]. The degree of change depends on the spectral overlap of the donor's emission and the acceptor's absorption with the LSPR as well as the position and orientation in FRET. Nitzan et al. theoretically predicted an enhancement of the energy transfer rate up to 6 orders of magnitude when a plasmonic metal material was introduced into a FRET system [55]. Reil and co-workers experimentally and theoretically found that the FRET depends on the interaction of donors and acceptors with plasmonic resonances in a nearby metallic nanoparticle [54]. The presence of LSPR modifies the decay rates of donor and acceptor. The electromagnetic field, in particular the near-field of a donor molecule, can excite the LSPR of a metallic nanoparticle, and thus leads to the decrease or increase of radiative and non-radiative rates. Finally, this changes the effective Förster radius and the FRET efficiency as well as the quantum yields of the donor and acceptor. Lakowicz et al. performed intensive investigations which are contributed to the FRET in the presence of plasmonic metals [56]. They found that FRET is efficiently enhanced by the plasmonic metal particles, and the apparent 
energy transfer distance increases with the size of silver particle and distance away from the metal. LSPR-enhanced FRET depends on the electromagnetic interaction of the donor and acceptor with the metal particle, and a large metal particle can result in a more efficient coupling of LSPR with the emission field.

The work mentioned above was mainly focused on the effect of plasmonic metals on the FRET process, and the metal particle plays an individual role of plasma supports in these works. Recently, it has been found that gold nanoparticles can also act as the energy acceptor, and its use as the acceptor in a RET system leads to more efficient energy transfer and longer detection distance in comparison with that of conventional organic dyes [37-40]. Strouse et al. conducted intensive fundamental work in this area. The spectral overlap between the donor and gold acceptor is determinant in the quenching of fluorescence of the donor [38,39]. Furthermore, Lee et al. suggested that the energy transfer quenching efficiency is governed by the absorption cross-section of the gold nanoparticles [57]. The larger sized gold nanoparticles result in more efficient quenching of fluorescence emission of QDs. However, understanding of the fluorescence quenching by the gold nanoparticle is still limited, and the intrinsic physical origin behind this system remains unclear. It is of fundamental and practical significance to further understand the energy transfer process containing gold nanoparticles with various LSPR characters, which will aid in the design of RET-based sensing devices for chemical and biological applications.

\subsection{Applications of SERS and RET approaches}

As described in Chapter 1, a wide range of applications of SERS and RET-based approaches can be found in biological warfare agent, detection of explosives (e.g., TNT), forensic science, 
environmental monitoring, food safety, industrial safety, chemical process, anti-bioterrorism and detection of counterfeiting. Research on nanostructured materials and the use of nanostructured arrays in these sensing strategies is addressing the need for better analyte selectivity and sensitivity. In addition, developing new nanostructures and their designs for SERS and RETbased sensors are important steps to realize the objective of miniaturized, inexpensive, portable, and on-site detection in a static and continuous manner in remote environments. The following part is devoted to giving an overview of the recent progress of SERS and fluorescent sensors, which addresses the development and use of new nanomaterials and nanostructures in particular.

\subsubsection{Application of SERS}

The discovery of SERS has re-inspired the interest of Raman spectroscopy not only as the structure analysis technique but also the chemical and biological sensing technique. Developing the SERS sensor is one of the hottest topics in chemistry, materials science, and life science. SERS has been used for detection of chemical and biological species at the single molecule level [58-61]. The material composition, size, shape and surface roughness impose great effects on the surface plasmon peak position, intensity and distribution, and thus affect the SERS signal [62]. In general, stronger electromagnetic field and the resulting higher SERS appear on the location close to the sharper and rougher surface. These structures with such SERS features are extremely desired.

SERS-based approaches have some advantages over fluorescent, SPR, and electrochemical ones [63], including (i) label-free detection, (ii) excellent reproducibility, (iii) more reliable multiplexing capability because of finger-printing Raman spectra, (iv) much higher sensitivity, and (v) potentially greater flexibility due to larger pools of available and non-overlapping Raman molecules. 


\section{(1) Nanoparticle-based SERS}

Local electromagnetic field is significantly enhanced at the gap between two particles, the socalled "hot spot", which allows SERS enhancement factor to increase up to $10^{12}-10^{15}$ [64]. Therefore, the nanostructures with sharp or rough junctions are preferable for SERS-based sensing. At present, the conventional approach is to obtain the condition-controllable aggregation of gold nanoparticles or silver nanoparticles. The Raman tags positioned at the "hot spot" can respond to the enhanced electromagnetic field, and thereby result in the SERS activity.

Highly SERS active nanostructures, including engineered dimers and trimers, can be obtained by the advanced synthetic chemistry and nanofabrication technique $[65,66]$. These homogeneous and reproducible structures further allow making high quality sensors with expected detection limit. Bell and Sirimuthu have used citrate-coated silver nanoparticles as the SERS substrate to determine the sequence of the DNA/RNA mononucleotides in the presence of $\mathrm{MgSO}_{4}$ [67]. The silver nanoparticles without any modification were just directly added to the solution containing mononucleotides. The cation $\mathrm{Mg}^{2+}$ induced the aggregation of the silver nanoparticles, and mononucleotides existing at the gap between the silver nanopartilces exhibited the enhanced Raman signal, leading to a detection limit at ppm level. Therefore, the direct injection of nanoparticles or silver nanoparticles into the biological cell resulted in the in situ detection of biomacromolecules. Lee et al. have developed a SERS-active platform through self-assembly of DNA-functionalized gold nanospheres and gold nanowires [68]. Gold nanoparticles were functionalized by Raman tag-labeled single strand DNA while gold nanowires were functionalized by another DNA. After adding the complementary DNA, one end of the target DNA bound to DNA adsorbed on the gold nanopsheres while the other end of target DNA bound to DNA on the gold nanowires. Thus, the gold nanosphere-DNA-gold nanowire sandwich 
architecture was formed to create the "hot spot". Raman signal from Raman tag existing in the "hot spot" was significantly enhanced. This system was demonstrated to be sensitive and specific for pathogen DNA targets with the detection limit at the $p \mathrm{M}$ level. It has a potential for diagnostics of infection diseases. The application of SERS for in vivo spectroscopic detection of tumor in live animals was investigated by Nie group, in which the small molecule tagged gold nanoparticles displayed brighter imaging for the rumor target than QDs [69]. In addition, the detection of explosive TNT is demonstrated by Ray group using cysteine-modified gold nanoparticles. TNT can form Meisenheimer complex with cysteine and displayed specific SERS signal under incident laser [70].

Nanostructures with high roughness, like nanostars, nanoflowers and nanotriangles, have also been used as SERS platforms, which bring high enhancement factors [71-73]. In addition, the composite materials with core/shell structures are able to amplify the local electromagnetic field, and even induce the fast, efficient electron transfer for the chemical enhancement of Raman signal [74]. However, the stability and the homogeneity of nanomaterials still remains a challenge, which may influence the reproducibility of Raman signals.

(2) Bead-based SERS sensors

Besides Raman tags were directly adsorbed onto the surfaces of nanospheres, nanowires and nanotubes, they are also embedded into beads for multiplex bioassay [63,75-77]. This brings in great advantages of: (i) long-term stability and reproducibility, (ii) less side reaction, (iii) great enhancement in signal of given analytes, (iv) facile surface functionalization for immobilization of biomolecules, and (v) no leakage of Raman tags.

Several approaches were used to obtain the beads that are encoded by multiple Raman tags for signal amplification. Raman tags were either encapsulated into the core or the shell layer. In Nie 
group's work, Raman tags (organic dyes) were incorporated into the $\mathrm{SiO}_{2}$ shell layer with a gold nanoparticle as the core [76]. The organic dyes containing the isothiocyanate group can be homogeneously dispersed into the $\mathrm{SiO}_{2}$ shell. The organic dye-engineered gold $/ \mathrm{SiO}_{2}$ core-shell nanomaterials could avoid the problems including surface adsorption, substrate variations and poor reproducibility, and paved a novel way for multiplex detection of biomarkers. The Raman tag-embedded $\mathrm{SiO}_{2}$ layer functionalized with carboxyl or amino groups can easily bind to biomolecules, and thereby are applied to the SERS-based biological imaging. Wang et al. have investigated the $\mathrm{Au} / \mathrm{SiO}_{2}$ beads for detection of pesticide residues on fruits, yeast cells and other small molecules [77]. In addition, Zhang et al. have found that the Raman tag-embedded $\mathrm{SiO}_{2}$ core-Au shell beads exhibited giant enhancement of SERS, which is probable ascribed to the fact that the nanoshell acts as a cavity to concentrate the electromagnetic field [78].

SERS-encoded hollow particles, which typically exhibit enhanced SERS, are another kind of efficient structure for biological assays. Alvarez-Puebla et al. have prepared gold nanoparticleencapsulated $\mathrm{SiO}_{2}$ hollow particles, which were encoded by benzenethiol, 4-nitrobenzenethiol or 4-hydroxybenzenethiol [64]. After adding the antibody-modified gold nanoparticles, the antibody-capsule conjugates were specifically bound to the antibody-Ag conjugates. Thereby the SERS signal from encoding species was obtained. Multiplex detection of biotargets can be realized with the mixed Raman tags. Six dissimilar DNA targets were simultaneously detected by the use of six Raman-labeled gold nanoparticle probes. The resulting SERS probes exhibited a detection limit of $20 f \mathrm{M}[79]$.

(3) Chip-based SERS sensors

A small area of chip can provide a SERS biosensing platform with multiplex detection capability and improved flexibility. Reich et al. have reported a sensing strategy based on SERS 
in order to detect the DNA sequence [80]. They added the DNA/silver nanoparticle conjugates to the DNA-functionalized silver film modified by Raman dyes. The smooth silver film produced only low SERS enhancement. After the DNA-modified silver nanoparticles were added, the single strand DNA complementarily bound to the DNA strand adsorbed on the silver film. As a result, the hot spots were created, leading to enhancement of the Raman signal.

Monitoring of chemical warfare agents and explosives is of great significance for homeland security in the current context of international anti-terrorism. Van Duyne group has investigated SERS biosensing for biowarefare agent using silver FONs for the first time [27,81]. The characteristic down-concave structure among those spheres provides the strong electromagnetic field for SERS. The detection of Bacillus subtilis spores was conducted through the extraction of calcium dipicolinate, a biomarker for bacillus spores. It was found that the limit of detection reached $2.1 \times 10^{-14} \mathrm{M}$, which was below the anthrax infectious dose of $10^{4}$ pores. In addition, the SERS spectra associated with the nanostructured metal substrate and various kinds of virus provides rapid detailed fingerprint information and released rapid and simple detection of virus [82].

Arrays of various nanopatterns have been used for SERS detection. These nanopatterns include nanoflowers, nanoholes, nanodots and nanotriangles of silver or gold on silicon or glass substrates, and are extremely stable $[21,83]$. Typically, the localized surface plasmon resonance absorption bands red-shift in order of sphere $<$ cylinder $<$ cube $<$ prism $<$ pyramid. The surface plasmon distribution surrounding the patterns varies as a function of its geometry. The strongest electromagnetic field appears near the sharp tip. Crozier and co-workers reported a doubleresonance SERS substrate, showing advantages over conventional single-resonance structures [84-86]. The double-resonance substrate consisted of a gold or silver disk array, a $\mathrm{SiO}_{2}$ spacer 
and a continuous gold film. The strong coupling interaction between localized surface plasmon and propagating surface plasmon contributed to the enhancement of the local field and the double-resonance feature in extinction spectra. The SERS enhancement factor of doubleresonance structure was more than two orders of magnitude higher than that of a gold disk array on the glass substrate. The largest SERS enhancement factors for the gold device and the gold device were $7.2 \times 10^{7}$ and $8.4 \times 10^{8}$, respectively. In addition, Bazan et al. have developed a SERS-active platform to detect multi-analytes simultaneously in a single bioassay [87]. The silver micropad was prepared on the silicon surface, and then functionalized with a mixture of three different aptamers. The three aptamers can specifically bind to three proteins, that is, human $\alpha$-thrombin, platetlet-derived growth factor-BB and immumoglobutin E, respectively. Three different Raman tags were chemically adsorbed onto the silver nanoparticle surface. After each protein was specifically bound onto the corresponding aptamer, three different Raman taglabeled silver nanoparticles were incubated in the aptamer-modified silver mircopad, and recognized by aptamers. Thus, the aptamer-silver mircopad/protein/Raman reporter-silver nanoparticle sandwiched architecture was formed, leading to the SERS signal. This sensor yielded a limit of detection of $100 \mathrm{pM}$.

\subsubsection{Application of RET-based fluorescent approaches}

Fluorescent sensors have been demonstrated to be useful for chemical sensing and bioimaging. Many contributions were carried out with respect to organic fluorescent sensors in the past decades. Since 1990s, inorganic semiconductor nanocrystals (quantum dots, QDs), metallic cluster fluorophores and organic-inorganic composites have emerged as novel fluorescent labels in biosensing and imaging, and are substituting for the conventional organic fluorophores. This is ascribed to great advantages of inorganic nanocrystals over the 
conventional organic dyes. Semiconducting QDs exhibit broad excitation profiles, narrow and symmetric emission spectra, high photostability and high quantum efficiency and excellent multiplex detection capability. For example, QDs with different emission wavelengths can be excited by single excitation source while organic dyes with different emission wavelengths must be excited by multiple excitation sources. Demand of simultaneous detection of more targets in single assay drives the development of inorganic nanocrystal-based fluorescent probes to replace organic fluorophores. Herein, we focus on the overview of inorganic fluorophores in the following part.

(1) Nanoparticle-based fluorescent sensors

Chalcogenide QDs are enxtensively used in fluorescent biosensors. However, they are toxic and hydrophobic. Recent development of synthetic chemistry has allowed preparing watersoluble QDs with mitigated toxicity [88-90]. The strategy developed for enabling biocompatibility is to modify the surface of QDs with 3-mercaptobenzoic acid (MPA), dihydrolipoic acid (DHLA), HS-poly(ethylene glycol)-carboxyl acid (HS-PEG-COOH), or other hydrophilic and bifunctional ligands.

The size-tunable optical properties allow QDs to act as optical acceptors as well as donors for biosensing based on Förster resonance energy transfer (FRET) [91,92]. Mattoussi group and Willner group have pioneered this area for detection of biomacromolecules, toxic metal ions and hazardous explosives, etc. Mattoussi and co-workers linked single-chain antibody fragments to the surface of DHLA-modified QDs (CdSe/ZnS core/shell) [93]. This antibody was pre-bound by a dye-labeled 2,4,6-trinitrotoluene (TNT) analogue. Exposure to the solution containing TNT resulted in displacement of TNT analogue pre-bound in the antibody binding site away from QDs, thereby the recovery of fluorescence emission of QDs. This system exhibited a limit of 
detection of $20 \mathrm{ng} / \mathrm{mL}$ for TNT. The excellent specificity of this assembly for TNT was demonstrated by examining three other analogues (Tetryl-DNT, 2,-A, 4,6-DNT and 2,6-DNT). It was reasonably expected that this system had the potential for multiplexed detection of targets through the use of QDs with different emission wavelengths. Willner and co-workers adopted the similar structured assembly to investigate hybridization and cleavage of DNA [94]. DNA/QDs conjugates were initially hybridized with the complementary Texas Red-labeled DNA. Addition of DNase I resulted in the cleavage of DNA, and successively the partial recovery of fluorescence emission of QDs.

Besides the organic dyes as the acceptor, gold nanoparticles can be used for the acceptor for RET, as mentioned above. Grant and co-workers have assembled the biosensor with fluorophores as the energy donor and gold nanoparticles as the energy acceptor, modified by specific capture antibody [95]. This assembling was found to be a feasible method for Porcine Reproductive and Respiratory Syndrome Virus (PRRSV) detection with the detection limit of 3 particle/ $\mu \mathrm{L}$. Furthermore, gold nanoparticles and silver nanoparticles with the size down to several atoms to 1-2 $\mathrm{nm}$ exhibited high photoluminescence quantum yields with respect to large particles and bulk materials $[96,97)$. Their fluorescence emission wavelength varies from blue to near-IR region. The emission wavelength undergoes a blue shift as the size of particles decreases. These fluorescent nanoparticles have been employed for detection of heavy metallic ion [96] and small molecules [97], showing the excellent selectivity for $\mathrm{Hg}^{2+}$ and a detection limit of $5.0 \mathrm{nM}$ in the presence of 2,6-pyridinedicarboxylic acid (PDCA). But less investigation was conducted to detect biomacromolecules both in vivo and in vitro, probably due to their unstable structure and difficult modification. 
Nanowire- and nanotube-based fluorescent biosensors are attractive for the optical bioassays because of their confined electron transportation along the one-dimension direction. Chen et al. have assembled a DNA aptamer probe through attaching thiolated thrombin binding aptamer on gold nanowires [98]. Exposure of these probes to the biotinylated thrombin led to the specific recognition of targets labeled by fluorescent reporter. The fluorescence intensity from the probetarget-reporter assembly varied as a function of the distance between the fluorophore and the gold nanowire due to the surface energy transfer. When the positive potential was applied to the gold nanowire, the fluorophore was attracted toward the nanowire, leading to the decrease of the fluorescence intensity. When the negative potential was applied to the nanowire, the fluorophore was repelled away from the surface, which resulted in the recovery of the fluorescence intensity from the fluorophore. This biosensor can detect the thrombin at a single molecule level with the limit of detection of $100 \mathrm{fM}$. It was believed that the electrically modulated fluorescence method can be extended to detection of other targets.

Novel composite nanomaterials, such as QD-carbon nanotubes and QD-graphene oxides, are really efficient candidates for fluorescent biosensors. Graphene oxide has been extensively studied for biosensors because of its unique characteristics such as facile surface modification, high mechanical strength, good water dispersibility, and photoluminescence [99]. The planar structure facilitates the electron and energy transportation in particular. In addition, graphene oxide is also a kind of ideal support for nanoparticle loading. Seo and co-workers have reported a graphene oxide-based immuno-biosensor for pathogen detection with high sensitivity and selectivity [99]. This sensor was based on the photoluminescence quenching of graphene oxides through the FRET process induced by the Au NPs. The antibody-modified graphene oxides could recognize the pathogen due to the specific antigen-antibody interaction. This system 
showed a detection limit of $10^{5} \mathrm{pfu} / \mathrm{mL}$, which was comparable with that of the conventional ELISA technique. Similarly, carbon nanotubes were demonstrated to be an efficient fluorescence quencher in biosensors [100]. The high stability and mechanical strength make carbon nanotubes feasible to be used in the stringent detection environment. Tan et al. have reported a hairpinstructured assembly, which consists of oligonucleotide-modified SWNTs and dyes-labeled complementary oligonucleotides [100]. Upon the complementary recognition of oligonuleotides, carbon nanotubes quenched the fluorescence emission from the dye tags.

(2) Bead-based fluorescent biosensors

Fluorophore-embedded beads can contain more than one fluorescent molecule in one particle to achieve high sensitive and high throughput analysis of targets. This strategy exhibits superior characteristics for bioassays [101-103], including: (i) avoiding the leakage of the fluorescent label and resulting long-term stability, (ii) enhancement of the fluorescence intensity and consequently the improved sensitivity, and (iii) facile surface functionalization for immobilization of biomolecules. Multiple detections can be realized through trapping the fluorophors with different emission spectra into single bead. The multiplex detection capacity depends on the spectral coding in the bead, which is determined by the intensity level and the color (emission wavelength) number. Theoretically, $n$ intensity levels with $m$ colors generate distinct $\left(n^{m}-1\right)$ codes [104].

Nie and co-workers have applied this approach to demonstrate the potential of the QDembedded beads for multiple spectral coding [104]. Different-sized QDs with different fluorescent emission were incorporated into the polymeric microbeads at the precisely controlled ratios. The dye-labeled DNA target was combined with the DNA-functionalized microbeads that contained the QDs at different concentration ratios. Thus the signals for multiple targets can be 
exhibited simultaneously at the single-bead level. This fluorescent platform can be used for high throughput detection of targets such as DNA, cancer biomarkers and heavy metals.

Nabiev et al. have explored the multiplex detection of circulating autoantibodies, the markers of systemic sclerosis [103]. The microbeads coded by three QDs with green, orange and red emissions were functionalized by the antigens, followed by coating of the bovine serum albumin protein. The specific interaction between the microbead and the antibody turned the fluorescence signal from the dye label "off" and "on" through the FRET mechanism. The results demonstrated the promising application of QD-encoded microbeads for multiplexed antibody profiling, clinical diagnostics of autoimmune diseases.

(3) Chip-based fluorescent biosensors

In two-dimensional bioassays, chips are usually patterned with various nano-scale or microscale structures. Chip-based fluorescent assays allow massive detection and screening of targets in a small area and can be integrated with microfuidics to build lab-on-chip systems. Alivisatos and co-workers have successfully deposited the QDs with specific fluorescence emission wavelength on the defined micrometer-sized surface to form the micropattern-containing QDs [106]. In this case, the micrometer-size gold pattern was grown on the top of a silicon wafer using the standard lithography technique. The gold pattern provided easy surface chemistry for DNA immobilization. Following this, the QDs of different emission wavelengths were functionalized by the corresponding complementary single strand DNA, respectively. Immersion of the Au patterned chip into the mixed DNA-QD conjugates resulted in the specific collection of QDs onto the corresponding Au patterns due to the specific interaction between the DNA-QD conjugate and the DNA-gold patterns. Although the authors' initial purpose was to sort the fluorescent nanocrystals, the resulting QD-coated gold patterns possessed the specific 
fluorescence emission, which is suitable for the multiplexed detection of biological targets at the nanometer scale.

Kim et al. have developed a novel biosensor for $\mathrm{Hg}^{2+}$ detection with a chip patterned by polydiacetylene [107]. The diacetylene molecules have an epoxy group that provides the versatile linkage chemistry for bioconjugation with biological molecules. The polydiacetylene-liposome microarray was obtained by the successive modification of glass substrate using amine functionality, amidation of epoxy liposomes, and linkage of single strand DNA aptamer. The red-phase polydiacetylene emitted the fluorescence that was dependent on the external stimuli. After addition of $\mathrm{Hg}^{2+}$, the formation of $\mathrm{T}-\mathrm{Hg}^{2+}-\mathrm{T}$ complexes caused the configuration change of the T-rich DNA aptamer, leading to the change in the fluorescence emission. This sensor exhibited the detection limit as low as $5 \mu \mathrm{M} \mathrm{Hg}^{2+}$.

\section{References}

[1] Ghosh, S. K.; Pal, T. Chem. Rev., 2007, 107, 4797-4862.

[2] Le Ru, E.C.; Etchegoin, P. G. Principles of Surface-Enhanced Raman Spectroscopy and related plasmonic effects, 1st ed.; Elsevier B. V.: Oxford, 2009.

[3] Liz-Marzán, L. M. Langmuir, 2006, 22, 32-41.

[4] Banaee, M. G.; Crozier, K. B. ACS Nano 2011, 5, 307-314.

[5] Mie, G. Ann. Phys., 1908, 25, 377.

[6] Schwartzberg, A. M.; Zhang, J. Z. J. Phys. Chem. C, 2008, 112, 10323-10337.

[7] Zhang, J.; Tang, Y.; Lee, K.; Ouyang, M. Nature, 2010, 466, 91-95.

[8] Cohen, R. W.; Cody, G. D.; Coutts, M. D.; Abeles, B. Phys. Rev. B, 1973, 8, 3689-3701.

[9] Wang, J.; Lau, W. M.; Li, Q. J. Appl. Phys., 2005, 97, 114303. 
[10] Ringe, E.; Zhang, J.; Langille, M. R.; Sohn, K.; Cobley, C.; Au, L.; Xia, Y.; Mirkin, C. A.; Huang, J.; Marks, L. D.; Van Duyne, R. P. Mater. Res. Soc. Symp. Proc., 2010, 1208, O10-02.

[11] Gole, A.; Murphy, C. J. Chem. Mater., 2004, 16, 3633-3640.

[12] Gou, L.; Murphy, C. J. Chem. Mater., 2005, 17, 3668-3672.

[13] Zhang, J.; Langille, M. R.; Personick, M. L.; Zhang, K.; Li, S.; Mirkin, C. A. J. Am. Chem. Soc., 2010, 132, 14012-14014.

[14] Zhang, Q.; Li, W.; Moran, C.; Zeng, J.; Chen, J.; Wen, L.-P.; Xia, Y. J. Am. Chem. Soc., 2010, 132, 11372-11378.

[15] Zhang, J.; Langille, M. R.; Mirkin, C. A. Nano Lett., 2011, 11, 2495-2498.

[16] Khoury, C. G.; Vo-Dinh, T. J. Phys. Chem. C, 2008, 112, 18849-18859.

[17] Hao, F.; Nehl, C. L.; Hafner, J. H.; Nordlander, P. Nano Lett., 2007, 7, 729-732.

[18] Nikoobakht, B.; El-Sayed, M. A. Chem. Mater., 2003, 15, 1957-1962.

[19] Lu, X.; Yavuz, M. S.; Tuan, H.-Y.; Korgel, B. A.; Xia Y. J. Am. Chem. Soc., 2008, 130, 8900-8901.

[20] Jensen, T. R.; Malinsky, M. D.; Haynes, C. L.; Van Duyne, R. P. J. Phys. Chem. B, 2000, 104, 10549-10556.

[21] Haes, A. J.; Haynes, C. L.; McFarland, A. D.; Schatz, G. C.; Van Duyne, R. P.; Zou, S. L. MRS Bull., 2005, 30, 368-375.

[22] Gopinath, A.; Boriskina, S. V.; Premasiri, W. R.; Ziegler, L.; Reinhard, B. M.; Negro, L. D. Nano Lett., 2009, 9, 3922-3929.

[23] Koerkamp, K. J. K.; Enoch, S.; Segerink, F. B.; van Hulst, N. F.; Kuipers, L. Phys. Rev. Lett., 2004, 92, 183901.

[24] Wang, J.; Zhou, H. S. Anal. Chem., 2008, 80, 7174-7178. 
[25] Wu, L.; Chu, H. S.; Koh, W. S.; Li, E. P. Opt Express, 2010, 18, 14395-14400.

[26] Yu, C.; Irudayaraj, J. Anal. Chem., 2007, 79, 572-579.

[27] Zhang, X.; Van Duyne, R. P. Mater. Res. Soc. Symp. Proc., 2005, 876E, R8.54.1-R8.54.6.

[28] Marinakos, S. M.; Chen, S.; Chilkoti, A. Anal. Chem., 2007, 79, 5278-5283.

[29] Jain, P. K.; Huang, W. El-Sayed, M. A. Nano. Lett., 2007, 7, 2080-2088.

[30] Lakowicz, J. R. Plasmonics, 2006, 1, 5-33.

[31] Lakowicz, J. R.; Ray, K.; Chowdhury, M.; Szmacinski, H.; Fu, Y.; Zhang, J.; Nowaczyk, K. Analyst, 2008, 133, 1308-1346.

[32] Lakowicz, J. R. Principles of Fluorescence Spectroscopy, 3nd ed.; Springer: Singapore, 2006.

[33] Medintz, I. L.; Clapp, A. R.; Mattoussi, H.; Goldman, E. R.; Fisher, B.; Mauro, J. M. Nat. Mater., 2003, 2, 630-638.

[34] Clapp, A. R.; Medintz,I. L.; Mauro, J. M.; Fisher, B. R.; Bawendi, M. G.; Mattoussi, H. J. Am. Chem. Soc., 2004, 126, 301-310.

[35] Rolinski, O. J.; Birch, D. J. S.; McCartney, L. J.; Pickup, J. C. Chem. Phys. Lett., 1999, 309, $395-401$.

[36] Clapp, A. R.; Medintz, I. L.; Mauro, J. M.; Fisher, B. R.; Bawendi, M. G.; Mattoussi, H. J. Am. Chem. Soc., 2004, 126, 301-310.

[37] Pons, T.; Medintz, I. L.; Sapsford, K. E.; Higashiya, S.; Grimes, A. F.; English, D. S.; Mattoussi, H. Nano Lett., 2007, 7, 3157-3164.

[38] Yun, C. S.; Javier, A.; Jennings, T.; Fisher, M.; Hira, S.; Peterson, S.; Hopkins, B.; Reich, N. O.; Strouse, G. F. J. Am. Chem. Soc., 2005, 127, 3115-3119.

[39] Jennings, T. L.; Singh, M. P.; Strouse, G. F. J. Am. Chem. Soc., 2006, 128, 5462-5467. 
[40] Ray, P. C.; Fortner, A.; Darbha, G. K. J. Phys. Chem. B, 2006, 110, 20745-20748.

[41] Gersten, J. I.; Nitzan, A. Surf. Sci. 1985, 158, 165-189.

[42] Griffin, J.; Singh, A. K.; Senapati, D.; Rhodes, P.; Mitchell, K.; Robinson, B.; Yu, E.; Ray, P. C. Chem. Eur. J., 2009, 15, 342-351.

[43] Singh, M. P.; Strouse, G. F. J. Am. Chem. Soc., 2010, 132, 9383-9391.

[44] Kambhampati, P.; Child, C. M.; Foster, M. C.; Campion, A. J. Chem. Phys., 1998, 108, 5013-5026.

[45] Schatz, G. C.; Van Duyne, R. P. Handbook of Vibrational Spectroscopy. John Wiley\&Sons Ltd., Chichester, 2002.

[46] García-Vidal, F. J.; Pendry, J. B. Phys. Rev. Lett., 1996, 77, 1163-1166.

[47]Xu, H.; Aizpurua, J.; Käll, M.; Apell, P. Phys. Rev. E, 2000, 62, 4318-4324.

[48] Persson, B. N. J.; Zhao, K.; Zhang, Z. Phys. Rev. Lett., 2006, 96, 207401.

[49] Yu, X.; Cai, H.; Zhang, W.; Li, X.; Pan, N.; Luo, Y.; Wang, X.; Hou, J. G. ACS Nano, 2011, $5,952-958$.

[50] Park, W.-H.; Kim, Z. H. Nano Lett., 2010, 10, 4040-4048.

[51] Morton, S. M.; Jensen, L. J. Am. Chem. Soc., 2009, 131, 4090-4098.

[52] Kinnan, M. K.; Chumanov, G. J. Phys. Chem. C, 2007, 111, 18010-18017.

[53] Lessard-Viger, M.; Rioux, M.; Rainville, L.; Boudreau, D. Nano Lett., 2009, 9, 3066-3071.

[54] Reil, F.; Hohenester, U.; Krenn, J. R.; Leitner, A. Nano Lett., 2008, 8, 4128-4133.

[55] Gersten, J.; Nitzan, A. J. Chem. Phys., 1980, 73, 3023-3037.

[56] Zhang, J.; Fu, Y.; Chowdhury, M. H.; Lakowicz, J. R. J. Phys. Chem. C, 2007, 111, 1178411792.

[57] Kondon, M.; Kim, J.; Udawatte, N.; Lee, D. J. Phys. Chem. C, 2008, 112, 6695-6699. 
[58] Nie, S.; Emory, S. R. Science, 1997, 275, 1102-1106.

[59] Lim, D. K.; Jeon, K. S.; Kim, H. M.; Nam, J. M.; Suh, Y. D. Nat. Mater., 2010, 9, 60-67.

[60] Dasary, S. S. R.; Singh, A. K.; Senapati, D.; Yu, H.; Ray, P. C. J. Am. Chem. Soc., 2009, $131,13806-13812$.

[61] Doering, W. E.; Piotti, M. E.; Natan, M. J.; Freeman, R. G. Adv. Mater., 2007, 19, 31003108.

[62] Braun, G.; Lee, S. J.; Dante, M.; Nguyen, T. Q.; Moskovits, M.; Reich, N. J. Am. Chem. Soc., 2007, 129, 6378-6379.

[63] Orendorff, C. J.; Gole, A.; Sau, T. K.; Murphy, C. J. Anal. Chem., 2005, 77, 3261-3266.

[64] Sanles-Sobrido, M.; Exner, W.; Rodríguez-Lorenzo, L.; Rodríguez-González, B.; CorreaDuarte, M. A.; Álvarez-Puebla, R. A.; Liz-Marzán, L. M. J. Am. Chem. Soc., 2009, 131, 26992705.

[65] Jackson, J. B.; Halas, N. J. PNAS, 2004, 101, 17930-17935.

[66] Li, W.; Camargo, P. H. C.; Lu, X.; Xia, Y. Nano Lett., 2009, 9, 485-490.

[67] Chen, G.; Wang, Y.; Yang, M.; Xu, J.; SJ Goh, Pan, M.; Chen, H. J. Am. Chem. Soc., 2010, $132,3644-3645$.

[68] Bell, S. E. J.; Sirimuthu, N. M. S. J. Am. Chem. Soc., 2006, 128, 15580-15581.

[69] Qian, X.; Peng, X.-H.; Ansari, D. O.; Yin-Goen, Q.; Chen, G. Z.; Shin, D. M.; Yang, L.; Young, A. N.; Wang, M. D. Nie, S. Nat. Bio., 2008, 26, 83-90.

[70] Dasary, S. S. R.; Singh, A. K.; Senapati, D.; Yu, H.; Ray, P. C. J. Am. Chem. Soc., 2009, $131,13806-13812$

[71] Kang, T.; Yoo, S. M.; Yoon, I.; Lee, S. Y.; Kim, B. Nano Lett., 2010, 10, 1189-1193. 
[72] Kang, T.; Yoon, I.; Jeon, K. S.; Choi, W.; Lee, Y.; Seo, K.; Yoo, Y.; Park, Q. H.; Ihee, H.; Suh, Y. D.; Kim, B. J. Phys. Chem. C, 2009, 113, 7492-7496.

[73] Camargo, P. H. C.; Rycenga, M.; Au, L.; Xia, Y. Angew. Chem. Int. Ed., 2009, 48, 2180 2184.

[74] Jena, B. K.; Ra, C. R. Chem. Mater., 2008, 20, 3546-3548.

[75] Zhou, Q.; Chao, Y.; Li, Y.; Xu, W.; Wu, Y.; Zheng, J. ChemPhysChem, 2007, 8, 921-925.

[76] Doering, W. E.; Nie, S. Anal. Chem., 2003, 75, 6171-6176.

[77] Li, J. F.; Huang, Y. F.; Ding, Y.; Yang, Z. L.; Li, S. B.; Zhou, X. S.; Fan, F. R.; Zhang, W.; Zhou, Z. Y.; Wu, D. Y.; Ren, B.; Wang, Z. L.; Tian, Z. Q. Nature, 2010, 464, 392-395.

[78] Zhang, P.; Guo, Y. J. Am. Chem. Soc., 2009, 131, 3808-3809.

[79] Cao, Y. W. C.; Jin, R.; Mirkin, C. A. Science, 2002, 297, 1536-1539.

[80] Braun, G.; Lee, S. J.; Dante, M.; Nguyen, T.-Q.; Moskovits, M.; Reich, N. J. Am. Chem. Soc., 2007, 129, 6378-6379.

[81] Zhang, X.; Young, M. A.; Lyandres, O.; Van Duyne, R. P. J. Am. Chem. Soc., 2005, 127, 4484-4489.

[82] Shanmukh, S.; Jones, L.; Driskell, J.; Zhao, Y.; Dluhy, R.; Tripp, R. A. Nano Lett., 2006, 6, 2630-2636.

[83] Yu, Q.; Guan, P.; Qin, D.; Golden, G.; Wallace, P. M. Nano Lett., 2008, 8, 1923-1928.

[84] Chu, Y.; Banaee, M. G.; Crozier, K. B. ACS Nano, 2010, 4, 2804-2810.

[85] Banaee, M. G.; Crozier, K. B. Opt. Lett., 2010, 35, 760-762.

[86] Chu, Y.; Crozier, K. B. Opt. Lett., 2009, 34, 244-246.

[87] Fabris, L.; Schierhorn, M.; Moskovits, M.; Bazan, G. C. small, 2010, 14, 1550-1557. 
[88] Susumu, K.; Uyeda, H. T.; Medintz, I. L.; Pons, T.; Delehanty, J. B.; Mattoussi, H. J. Am. Chem. Soc., 2007, 129, 13987-13996.

[89]. Dixit, S. K.; Goicochea, N. L.; Daniel, M. C.; Murali, A.; Bronstein, L.; De, M.; Stein, B.; Rotello, V. M.; Kao, C. C.; Dragnea, B. Nano Lett., 2006, 6, 1993-1999.

[90] Jiang, W.; Mardyani, S.; Fischer, H.; Chan, W. C. W. Chem. Mater., 2006, 18, 872-878.

[91] Dennis, A. M.; Bao, G. Nano Lett., 2008, 8, 1439-1445.

[92] Roy, R.; Hohng, S.; Ha, T. Nat. Method, 2008, 5, 507-516.

[93] Goldman, E. R.; Medintz, I. L.; Whitley, J. L.; Hayhurst, A.; Clapp, A. R.; Uyeda, H. T.; Deschamps, J. R.; Lassman, M. E.; Mattoussi, H. J. Am. Chem. Soc., 2005, 127, 6744-6751.

[94] Gill, R.; Willner, I.; Shweky, I.; Banin, U. J. Phys. Chem. B, 2005, 109, 23715-23719.

[95] Stringera, R. C.; Schommerb, S.; Hoehna, D.; Grant, S. A. Sensor Actuat. B: Chem., 2008, $134,427-431$.

[96] Huang, C. C.; Yang, Z.; Lee, K. H.; Chang, H. T. Angew. Chem. Int. Ed., 2007, 46, 6824 6828.

[97] Shang, L.; Dong, S. Biosens. Bioelectro., 2009, 24, 1569-1573.

[98] Huang, S.; Chen, Y. Nano Lett., 2008, 8, 2829-2833.

[99] Jung, J. H.; Cheon, D. S.; Liu, F.; Lee, K. B.; Seo, T. S. Angew. Chem. Int. Ed., 2010, 49, 15.

[100] Yang, R.; Jin, J.; Chen, Y.; Shao, N.; Kang, H.; Xiao, Z.; Tang, Z.; Wu, Y.; Zhu, Z.; Tan, W. J. Am. Chem. Soc., 2008, 130, 8351-8358.

[101] Li, J.; Zhao, X.W.; Zhao, Y. J.; Gu, Z. Z. Chem. Commun., 2009, 2329-2331.

[102] Vaidya, S. V.; Gilchrist, M. L.; Maldarelli, C.; Couzis, A. Anal. Chem., 2007, 79, 85208530. 
[103] Sukhanova, A.; Susha, A. S.; Bek, A.; Mayilo, S.; Rogach, A. L.; Feldmann, J.; Oleinikov, V.; Reveil, B.; Donvito, B.; Cohen, J. H. M.; Nabiev, I. Nano Lett., 2007, 7, 2322-2327.

[104] Han, M.; Gao, X.; Su, J. Z.; Nie, S. Nat. Biotech., 2001, 19, 631-635.

[105] Li, Z.; Zhang, Y.; Jiang, S. Adv. Mater., 2008, 20, 1-5.

[106] Gerion, D.; Parak, W. J.; Williams, S. C.; Zanchet, D.; Micheel, C. M.; Alivisatos, A. P. J. Am. Chem. Soc., 2002, 124, 7070-7074.

[107] Lee, J.; Jun, H.; Kim, J. Adv. Mater., 2009, 21, 3674-3677. 


\section{CHAPTER 3: CHARGE TRANSFER INDUCED ELECTROMAGENTIC ENHANCEMENT OF SURFACE RAMAN SCATTERING}

\subsection{Introduction}

Surface-enhanced Raman scattering (SERS) possesses a promising application in a wide range of fields including environmental monitoring, chemical and biological sensing, and food safety, etc.[1,2]. This is mainly due to its superior characteristics of high sensitivity (e.g. single molecule level), multiplexing detection capability, and simple and inexpensive manipulation [36]. Fundamental understanding of physical essence for the SERS process is necessary to develop the SERS device in various applications. It is well-known that the remarkable enhancement of Raman signals arises from the chemical enhancement (CE) and the electromagnetic enhancement (EM) mechanisms [7-10]. Both the mechanisms co-exist in most of SERS systems but the contribution of CE mechanism is much lower than that of EM enhancement $[4,11,12]$. The CE is mainly ascribed to the charge transfer between the metal and the chemisorbed analyte molecules $[4,13]$. The EM enhancement is due to the excitation of significant localized surface plasmon resonance (LSPR) absorption in the local electric field on the surface of the nanostructures, which results from the interactions between the metallic nanostructures and the incident electromagnetic field. Co-existence of the $\mathrm{CE}$ enhancement with the EM enhancement makes the mechanistic investigation difficult in the SERS system, and their respective roles in the SERS process are difficultly distinguished. 
As introduced in Chapter 2, Raman signals of all molecules positioning in the "hot spots" are significantly amplified based on the EM mechanism. In the CE enhancement, the charge transfer process is dependent on the molecular structure, as demonstrated by previous studies $[4,14]$. Conjugated molecules are preferable for electron transfer [14], so the chemical enhancement in SERS due to the charge transfer is supposed to be dependent on the molecular structure. That is, Raman signals from different molecules can be selectively enhanced to different extents in the charge transfer mechanism. Chumanov et al. reported an effect of plasmon-induced electronic coupling in silver nanoparticle arrays on silver mirror film with various organic molecules [15]. It was indicated that the cross-effect between LSPR and the molecular structure can be found in the SERS process. However, limited investigations on effects of molecular structure on chemical enhancement mechanism in SERS and their interactions with electromagnetic field are available. To further understand the CE and EM enhancement mechanisms in SERS, a systematic investigation on SERS of various structural molecules on different LSPR substrates is necessary.

In this chapter, the presented work is focused on the SERS of different molecules (e.g. linear and planar) on various sized gold nanoparticles. Contribution of charge transfer to the electromagnetic enhancement of SERS of these organic molecules is investigated.

\subsection{Materials and experimental methods}

\subsubsection{Chemicals}

Chloroauric acid trihydrate $\left(\mathrm{HAuCl}_{4} \cdot 3 \mathrm{H}_{2} \mathrm{O}\right)$, thiophenol (TP), (L)-cysteine (Cys) (98+\%), trisodium citrate dehydrate $\left(\mathrm{Na}_{3} \mathrm{C}_{6} \mathrm{H}_{5} \mathrm{O}_{7} \cdot 2 \mathrm{H}_{2} \mathrm{O}\right.$, ACS, 90.0+\%) and hydrochloric acid standard solution $(1.0 \mathrm{~N})$ were purchased from Alfa Aesar. $p$-mercaptobenzoic acid (MBA, Technical 
grade 90\%), 3-mercaptopropionic acid (MPA, 99+\%) and $\mathrm{Hg}\left(\mathrm{NO}_{3}\right)_{2}$ were purchased from Sigma-Aldrich. $80 \mathrm{~nm}$ sized gold nanoparticles (NPs) were purchased from BioAssay Works Naked Gold (USA). All materials were used as received. All solvents were obtained from the commercial sources and used without further purification. Deionized (D.I.) water produced by the Milli-Q Integral 3/5/10/15 system (18.2 M $/ \mathrm{cm}^{2}$, Millipore Corp., USA) was used for the preparation of all the solutions. All glassware was successively cleaned with nitric acid and D.I. water, and then dried before use.

\subsubsection{Synthesis of gold nanoparticles}

The mono-dispersed gold colloids in a diameter of $15 \mathrm{~nm}$ were synthesized by the wellestablished citric reduction method [16-18]. Typically, an aliquot of $50 \mathrm{~mL}$ aqueous solution of chloroauric acid trihydrate $(0.4 \mathrm{mM})$ was heated to boiling under magnetic stirring. $2 \mathrm{~mL}$ trisodium cirate dihydrate $(1 \% \mathrm{wt})$ aqueous solution was then added. As the reaction time prolonged, the solution underwent a series of color changes, and finally became wine red. The solution was held at boiling for $1 \mathrm{~h}$, and then naturally cooled to room temperature. The molar concentration was calculated by UV-Visible absorption spectroscopy based on the Beer-Lambert law using the molar extinction coefficient of $1 \times 10^{8}(\mathrm{M} \cdot \mathrm{cm})^{-1}[19]$. Generally, the concentration of the aqueous solution was $12.0 \mathrm{nM}$.

Gold nanoparticles in diameter of $3 \mathrm{~nm}$ were synthesized by the modified Brust-Schiffrin method [20]. Typically, $0.5 \mathrm{mmol}(0.197 \mathrm{~g})$ of $\mathrm{HAuCl}_{4} \cdot 3 \mathrm{H}_{2} \mathrm{O}$ dissolved in a $5 \%(\mathrm{w} / \mathrm{v})$ aqueous solution will be mixed with $1.5 \mathrm{mmol}$ of MBA or MPA in $100 \mathrm{~mL}$ methanol to give a transparent solution in a $250 \mathrm{~mL}$ flask. $15 \mathrm{~mL} \mathrm{NaOH}$ aqueous solution $(0.3 \mathrm{M})$ was then added. Subsequently freshly prepared $25 \mathrm{~mL}$ aqueous sodium borohydride $\left(\mathrm{NaBH}_{4}\right)$ solution $(0.2 \mathrm{M})$ was added at a rate of $5 \mathrm{~mL}$ per min with vigorous stirring under Ar flow in an ice bath. The 
solution turned dark-brown immediately. Further addition of the reductant led to a dark-brown precipitate. After further stirring for $30 \mathrm{~min}$, the solvent was removed after centrifuging at 3,000 rpm for $5 \mathrm{~min}$. The precipitate was then washed twice with a $20 \%(\mathrm{v} / \mathrm{v})$ water/methanol solution through an ultrasonic redispersion-centrifugation process to remove the inorganic or organic impurities. This process was repeated with $99.8 \%$ methanol to remove unbound thiols. As a result, MBA- or MPA-functionalized gold nanoparticles were obtained and stored at $4{ }^{\circ} \mathrm{C}$ for further use.

\subsubsection{Modification of gold nanoparticles}

As-prepared $15 \mathrm{~nm}$ sized gold nanoparticles were modified by MBA, MPA, Cys or their mixture with TP, respectively. A stock solution of these ligands $(3.0 \mathrm{mM}))$ was prepared in ethanol. $1 \mathrm{~mL}$ ethanolic stock solution of MBA, MPA or Cys was added to $15 \mathrm{~mL}$ of the crude gold nanoparticle aqueous solution, heated up to $40-50{ }^{\circ} \mathrm{C}$ to evaporate ethanol, and then magnetically stirred at room temperature for $2 \mathrm{~h}$. For modification by the mixed ligands with MPA and TP, ethanolic stock solutions with various MPA to TP ratios were added to the asprepared $15 \mathrm{~nm}$ gold nanoparticle aqueous solution, respectively.

As-prepared $3 \mathrm{~nm}$ sized gold nanoparticles were modified by MBA or MPA. In the case where MBA is absent, we used gold nanoparticles prepared in the MPA ligand, but in the case where MBA is present, the gold nanoparticles prepared in the MBA ligand were used. Typically, $2.5 \mathrm{mg} 3 \mathrm{~nm}$ gold nanoparticles were added to $100 \mathrm{~mL}$ D.I. water and held for at least $30 \mathrm{~min}$ to get a $12 \mathrm{nM}$ gold nanoparticle solution. Subsequently $1 \mathrm{~mL}$ MPA, TP and MBA ethanolic solution was added to $15 \mathrm{~mL}$ aqueous solution of gold nanoparticles, respectively. For the modification of the mixed ligands with MPA and TP, the stock solutions with various MPA to 
TP ratios were added to the as-prepared $3 \mathrm{~nm}$ gold nanoparticle solution functionalized by MPA. The solution was incubated overnight for future use.

The $80 \mathrm{~nm}$ sized gold nanoparticles were modified by MBA as follows. $1 \mathrm{~mL}$ MBA ethanolic solution $(3.0 \mathrm{mM})$ was added to $15 \mathrm{~mL}$ aqueous solution of $80 \mathrm{~nm}$ gold nanoparticles with the concentration of $12 \mathrm{nM}$. The rest of the procedure was similar to that for modification of $15 \mathrm{~nm}$ sized gold nanoparticles.

\subsubsection{Creation of ligand-modified gold nanoparticle aggregation}

$1 \mathrm{~mL}$ of the $10 \mathrm{mM} \mathrm{Hg}^{2+}$ solution was added to $1 \mathrm{~mL}$ of the surface-modified gold nanoparticle aqueous solution to obtain aggregates of gold nanoparticles.

In the case of different $\mathrm{pH}$ values, the $\mathrm{pH}$ value was adjusted by addition of $\mathrm{KOH}(5 \mathrm{M})$ or $\mathrm{HCl}(1 \mathrm{M})$. In this work, 4 different 4-MBA modified $15 \mathrm{~nm}$ gold nanoparticle samples with the $\mathrm{pH}$ value of $1.5,4.7,6.3$ and 10.1 were prepared.

\subsubsection{Characterization}

The size of gold nanoparticles was observed under a transmission electron microscope (TEM) with a JEM $2100 \mathrm{~F}$ operated at $200 \mathrm{kV}$. The TEM specimens were prepared by dropping the gold nanoparticle solution onto a carbon-coated copper grid, and then dried in air. UV-visible absorption spectra were recorded in the range of $200-800 \mathrm{~nm}$ with the Shimadzu UV-2550 spectrometer (Japan). The Fourier transform infrared (FTIR) spectra were obtained under the transmission mode by using KBr pellet method in a Thermo Nicolet 6700 spectrometer. Raman spectra were collected using a Renishaw invia Raman spectrometer at the excitation lasers of $514.5 \mathrm{~nm}$ or $532 \mathrm{~nm}$. A microscope equipped with $20 \times$ objective was used to focus the incident excitation laser. The laser power on the sample was $50 \mathrm{~mW}$ (or $500 \mathrm{~mW}$ ). For Raman 
measurement, a drop of solution was placed onto the glass slide. Three spectra from different sites were collected. The averaged spectra were obtained to represent the SERS results.

\subsection{Creation of "hot spots" and experimental principle}

Four commonly used small molecules, which are MBA, MPA, TP and Cys, were used, respectively (Figure 3.1a). These molecules possess planar structures and linear structures, and can bind onto the gold surface through the S-Au bond [21,22]. Figure $3.1 \mathrm{~b}$ schematically shows the formation of gold nanoparticle aggregates in the present of $\mathrm{Hg}^{2+}$. Firstly, the gold nanoparticles were modified by small molecules mentioned above (e.g. MBA, MPA or Cys). The modified gold nanoparticles can be well-dispersed into the water solution, and thus less or negligible "hot spots" contribute to the SERS. Then, addition of $\mathrm{Hg}^{2+}$ brings in gold nanoparticles close to each other through the coordination chemistry between $\mathrm{Hg}^{2+}$ and carboxylic group [23-25]. FTIR and TEM demonstrated the formation of gold nanoparticle aggregates as well (Figure 3.2). It can be seen that MBA 
(a)

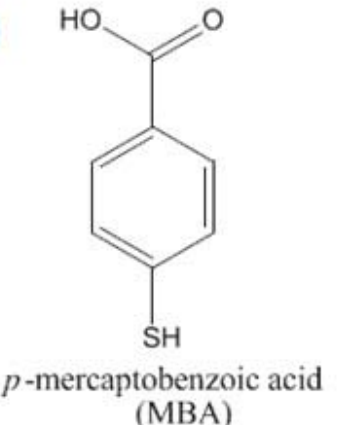<smiles>CSc1ccccc1</smiles>

thiophenol (TP)

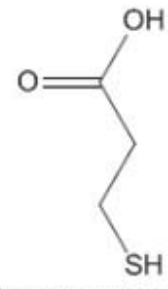

3-mercaptopropoinic acid

(MPA)

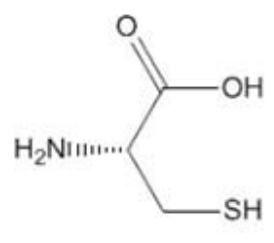

(L)-cysteine (Cys)

(b)
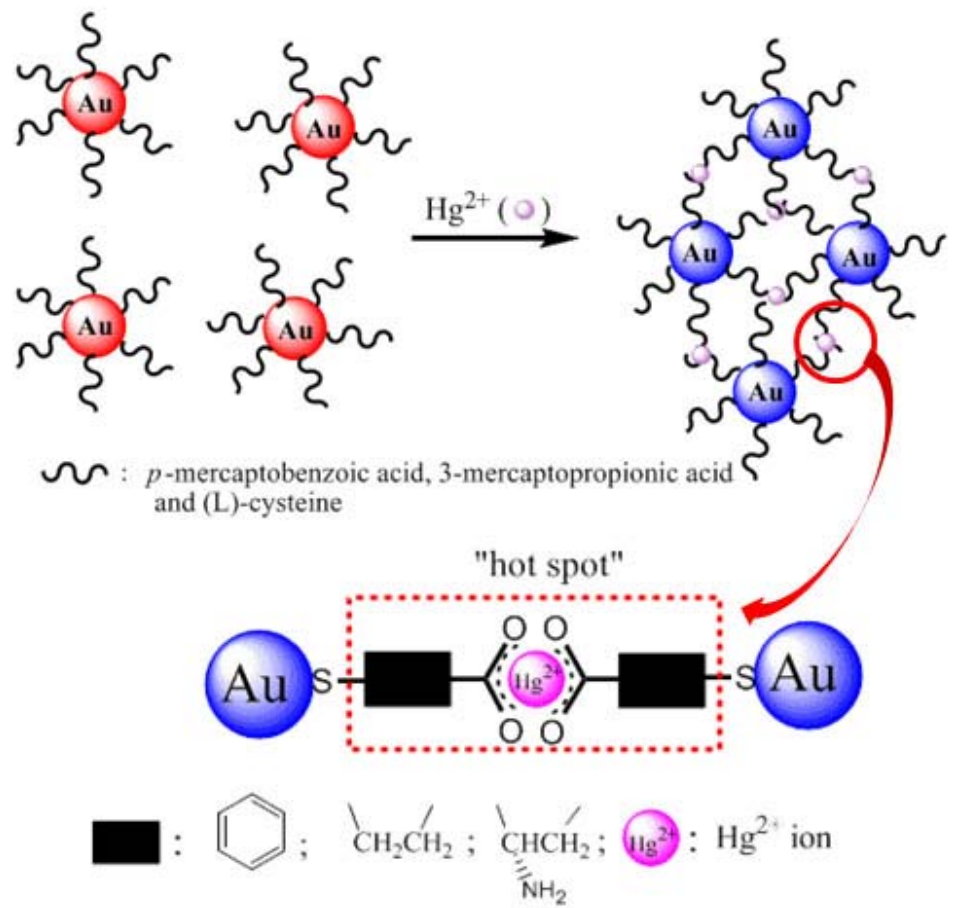

Figure 3.1 (a) Molecular structures of ligands used. (b) Schematic illustration of "hot spots" formed by the coordination chemistry between divalent ion (e.g. $\left.\mathrm{Hg}^{2+}\right)$ and carboxylic group.

shows an absorption peak around $2550 \mathrm{~cm}^{-1}$, which is assigned to the $-\mathrm{S}-\mathrm{H}$ bond [26]. Nevertheless, in the case of MBA-gold nanoparticles, the peak around $2550 \mathrm{~cm}^{-1}$ disappeared, which is attributed to formation of the S-Au bond [27]. On the other hand, the $\mathrm{C}=\mathrm{O}$ peak around $1720 \mathrm{~cm}^{-1}$ disappeared after addition of $\mathrm{Hg}^{2+}$. This is mainly due to the dissolution of $\mathrm{C}=\mathrm{O}$ band 

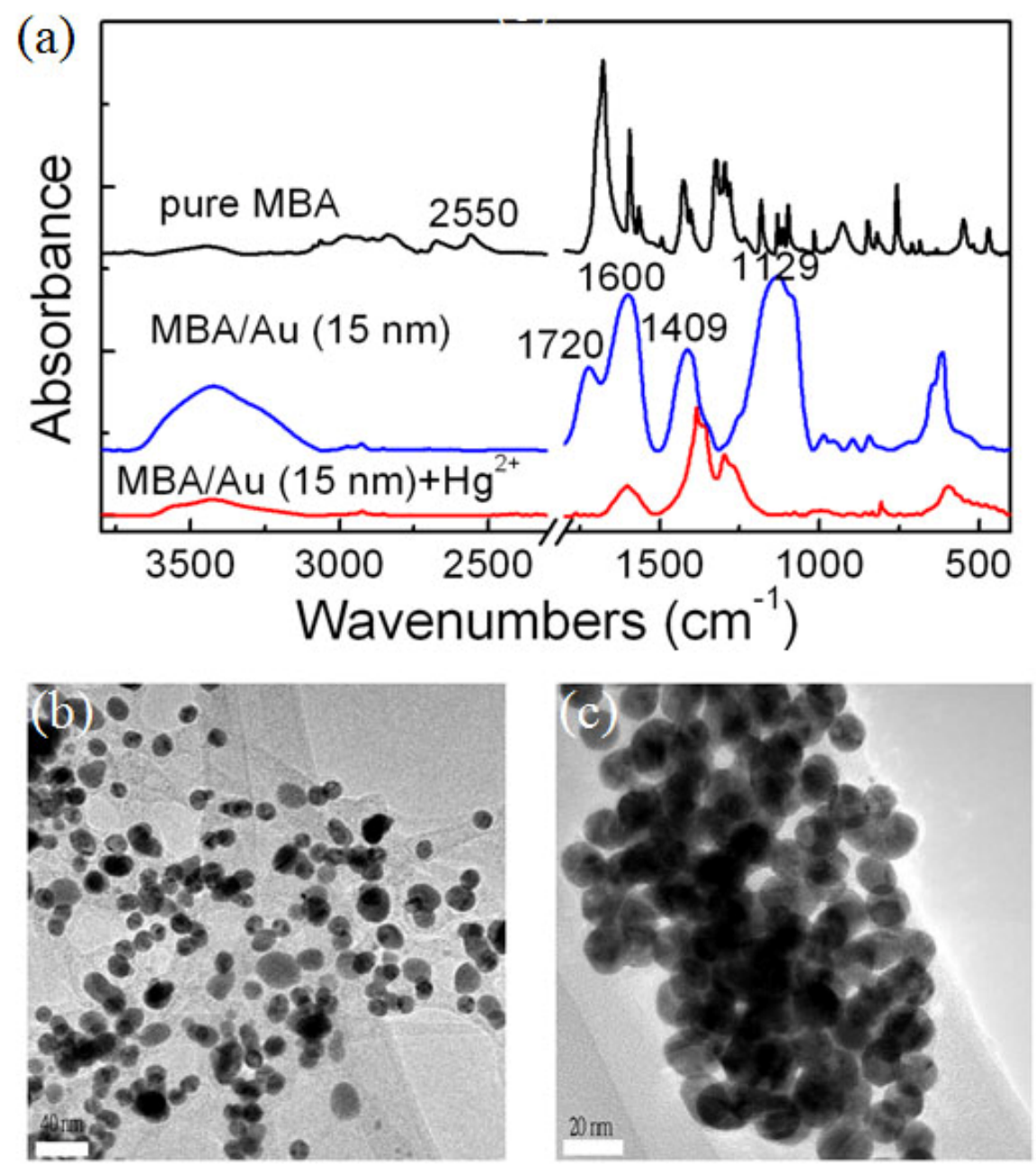

Figure 3.2 (a) FTIR of pure $p$-mercaptobenzoic acid, $p$-mercaptobenzoic acid-modified gold nanoparticles with $15 \mathrm{~nm}$ in diameter before and after $\mathrm{Hg}^{2+}$ addition. TEM images of $p$ mercaptobenzoic acid-modified gold nanoparticles (b) before and (c) after $\mathrm{Hg}^{2+}$ addition.

through the coordination of $\mathrm{Hg}^{2+}$ toward $\mathrm{COOH}$ group (Figure 3.1b). Therefore, the present results indicate that modification of gold nanoparticles and addition of $\mathrm{Hg}^{2+}$ enable the generation of gold nanoparticle aggregates. 


\subsection{SERS of different structural molecules}

Figure 3.3 shows the UV-visible absorption spectra and the corresponding SERS spectra of various ligands on $15 \mathrm{~nm}$ gold nanoparticles under the excitation of $514.5 \mathrm{~nm}$ laser. It can be seen that the absorption at longer wavelength is much stronger in solutions containing the $\mathrm{Hg}^{2+}$ than that without $\mathrm{Hg}^{2+}$ for all ligand-modified gold nanoparticles. This is because gold nanoparticle aggregates form in the presence of $\mathrm{Hg}^{2+}$, which is consistent with the results in the previous work $[28,29]$. The SERS band around $1045 \mathrm{~cm}^{-1}$ is assigned to the vibration mode of the aromatic ring. The bands around $1125 \mathrm{~cm}^{-1}$ and $1282 \mathrm{~cm}^{-1}$ are attributed to deformation modes of $\mathrm{C}-\mathrm{H}$. The $881 \mathrm{~cm}^{-1}$ and $1457 \mathrm{~cm}^{-1}$ peaks correspond to the deformation mode $\delta\left(\mathrm{COO}^{-}\right)$ and the stretching mode $v_{\mathrm{s}}\left(\mathrm{COO}^{-}\right)$, respectively [30,31]. The SERS signal from the gold aggregates in the presence of $\mathrm{Hg}^{2+}$ is much stronger than that from the dispersed gold nanoparticles in the absence of $\mathrm{Hg}^{2+}$. Such SERS enhancement is ascribed to the created "hot spots" between proximal gold nanoparticles in the aggregates due to the coupling of dipole plasmon $[32,33]$. Furthermore, it should be noted that MBA displays much more SERS active than MPA and Cys on the gold nanoparticle surface. We attribute this to the difference between the planar aromatic structure of MBA and the linear structure of MPA and Cys, which results in different electronic structures and electronic states in essence. As is well-known, the charge transfer occurs between gold nanoparticles and aromatic molecules due to the small gap between the Fermi level of gold and the LUMO level of aromatic molecules [14].

\subsection{SERS in different thiophenol to 3-mercaptopropionic acid ratios}

In order to investigate origin of the strong SERS activity in MBA-modified gold 

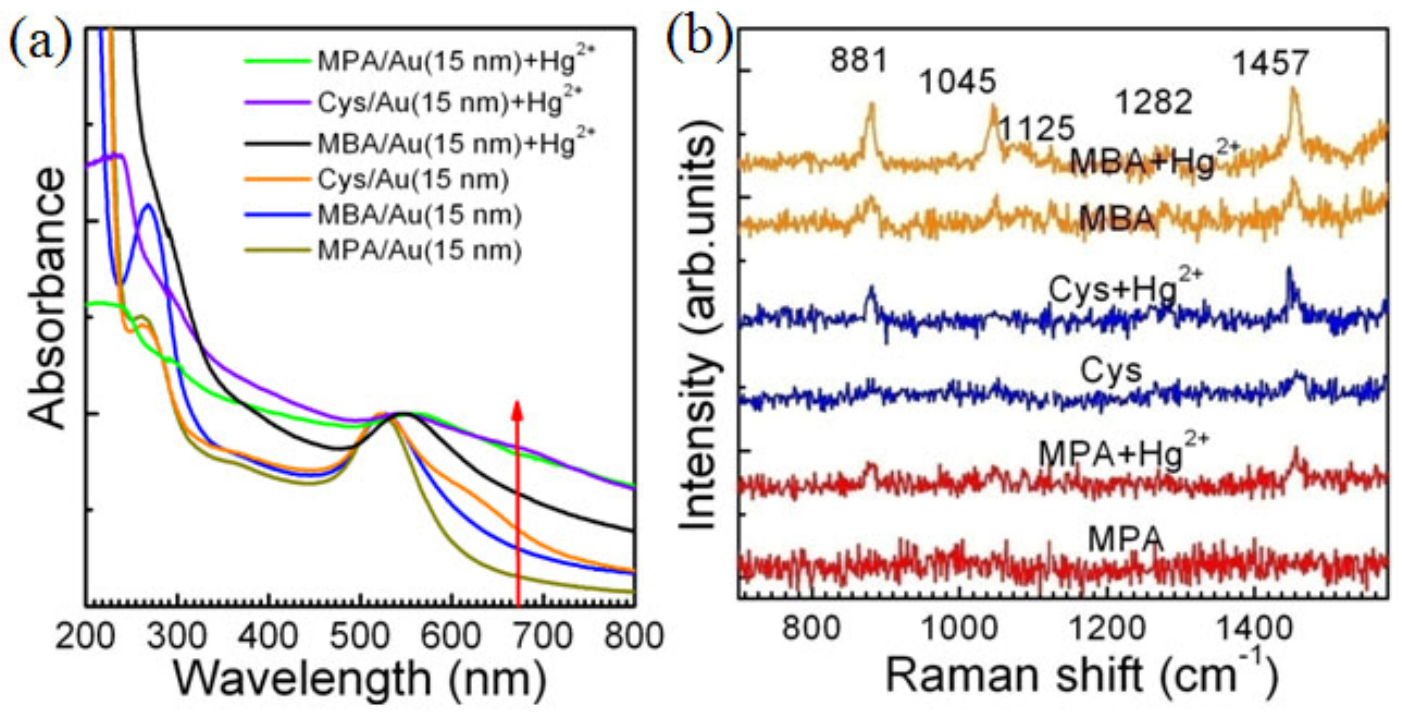

Figure 3.3 (a) UV-visible absorption spectra and (b) SERS spectra obtained from the aqueous solution of surface-modified gold nanoparticles $(15 \mathrm{~nm})$ before and after $\mathrm{Hg}^{2+}$ addition. $(6 \mathrm{nM}$ gold nanoparticles, $0.2 \mathrm{mM}$ ligand, $5 \mathrm{mM} \mathrm{Hg}^{2+}$ and $\mathrm{pH}$ value=6.3).

nanoparticles, we chose two typical molecules: TP and MPA. TP has a benzene ring with a thiol group, similar to MBA, and MPA has a linear structure with $\mathrm{COOH}$ group. So addition of $\mathrm{Hg}^{2+}$ can result in the aggregation of gold nanoparticles (Figure 3.4). Figure 3.5 shows the Raman spectra of TP and MPA co-modified gold nanoparticles under different TP/MPA ratios. It can be seen that in the case of only MPA, the weak deformation mode $\delta\left(\mathrm{COO}^{-}\right)$and the weak stretching mode $v_{\mathrm{s}}\left(\mathrm{COO}^{-}\right)$at $881 \mathrm{~cm}^{-1}$ and $1457 \mathrm{~cm}^{-1}$ are observed. After gold nanoparticles are comodified by TP and MPA, there are characteristic aromatic vibration mode and $\mathrm{C}-\mathrm{H}$ vibration modes observed in the SERS spectra. More importantly, it should be noted that Raman peaks around $881 \mathrm{~cm}^{-1}$ and $1457 \mathrm{~cm}^{-1}$ are significantly enhanced with the increasing TP/MPA ratio. As shown above, TP has no $\mathrm{COOH}$ group, and cannot bind to $\mathrm{Hg}^{2+}$ through the coordination chemistry. Aggregation of gold nanoparticles is attributed to the coordination bond formation 
between $\mathrm{Hg}^{2+}$ and carboxylic group in MPA. The present results indicate that the increasing TP/MPA ratio enhances not only the Raman peaks from TP but aslo the SERS of carboxylic group in MPA in despite of the reduced absolute concentration of MPA. It is suggested that the charge transfer can occur from the gold nanoparticle to TP, and can delocalize in the outer layer to form the free carriers. These delocalized electrons can behave like these free electrons in metals, which contribute to the electromagnetic enhancement of SERS of MPA, The peak intensity of $1457 \mathrm{~cm}^{-1}$ first increases with the increase of TP/MPA ratio, and then reaches saturation (Figure 3.5b).
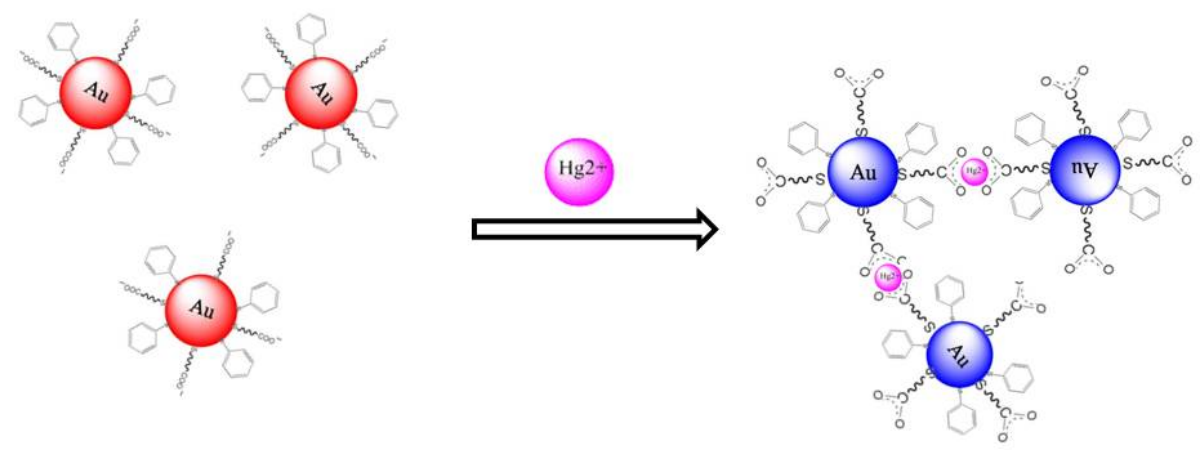

Figure 3.4 Schematic aggregation of the mixed ligand-modified gold nanopartilces in the presence of $\mathrm{Hg}^{2+}$.

\subsection{SERS in various thiophenol amounts}

Figure 3.6 shows SERS spectra of the mixture of TP and MPA on $15 \mathrm{~nm}$ gold nanoparticles. The MPA concentration is kept constant while the TP concentration increases. It can be clearly seen that not only peaks from benzene ring and $\mathrm{C}-\mathrm{H}$ from TP increase in the intensity but also the peak intensities from $\mathrm{COO}^{-}$in MPA increase with the increasing TP concentration. This 
demonstrates again that the existence of TP on the gold nanopartice surface benefits to the SERS enhancement of MPA.

(a)

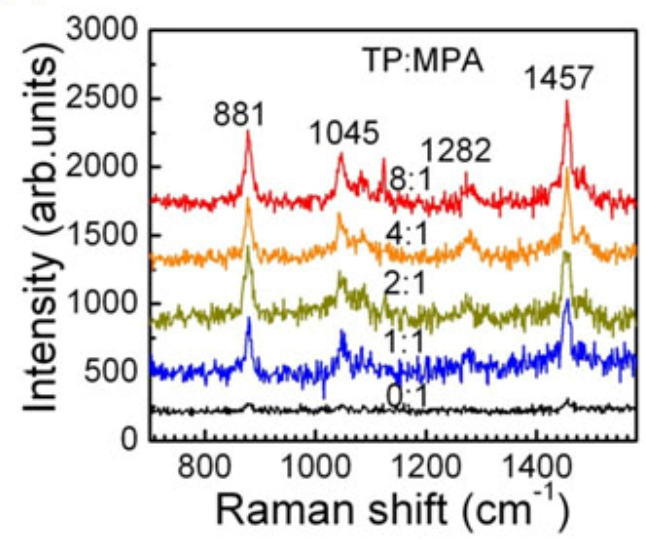

(b)

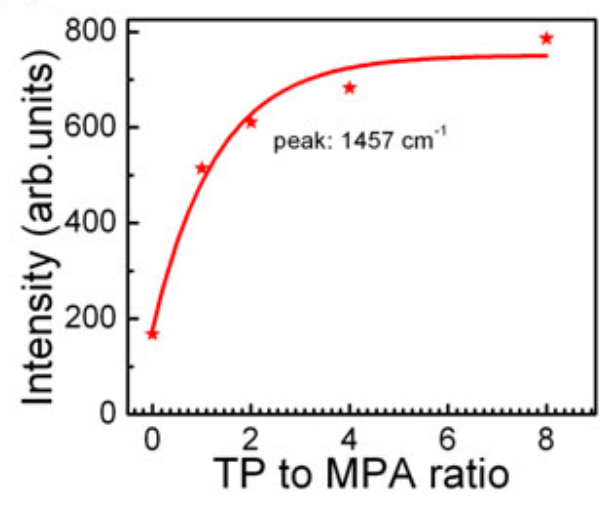

Figure 3.5 (a) SERS spectra of solutions containing different thiophenol/3-mercaptopropionic acid ratios on $15 \mathrm{~nm}$ gold nanoparticles. (b) The intensity of SERS peak (at $1457 \mathrm{~cm}^{-1}$ ) as a function of the thiophenol/3-mercaptopropionic acid molar ratio. ( $6 \mathrm{nM}$ gold nanoparticles, 0.2 $\mathrm{mM}$ ligands (TP+ MPA), $5 \mathrm{mM} \mathrm{Hg}^{2+}$ and $\mathrm{pH}=6.3$ ).

\subsection{SERS in gold nanoparticles with various LSPR absorptions}

There is no doubt that in the present SERS system, both CE and LSPR-induced EM mechanisms contribute to the SERS enhancement. As mentioned in Chapter 2, all molecules should be enhanced in the EM enhancement, and show the different enhancement factors. The EM enhancement mechanism cannot explain the difference among these molecules used. We propose that the chemical enhancement is involved and results in the significant difference in the present work. In order to confirm the proposed mechanism, we investigated the SERS activity of 
MBA adsorbed on different sized gold nanoparticles with negligible or strong LSPR absorption at different positions. 3, 15 and $80 \mathrm{~nm}$ gold nanoparticles were used (Figure 3.7). The $3 \mathrm{~nm}$ sized

(a)

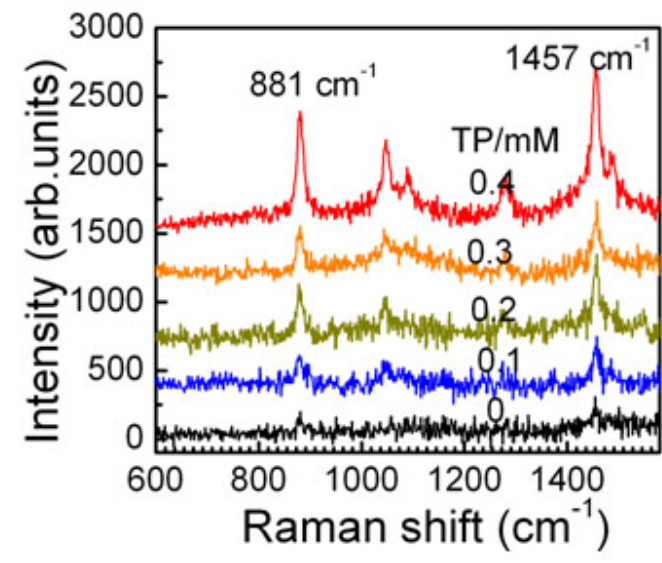

(b)

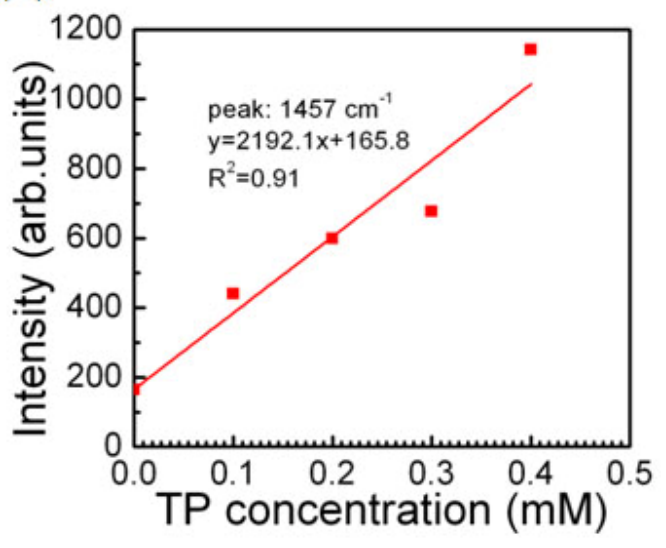

Figure 3.6 (a) SERS spectra of the mixture of thiophenol and 3-mercaptopropoinic acid adsorbed on the $15 \mathrm{~nm}$ sized gold aggregate. (b) The intensity of SERS peak (at $1457 \mathrm{~cm}^{-1}$ ) as a function of the thiophenol molar concentration. The thiophenol molar concentration increased but the 3-mercaptopropoinic acid molar concentration was kept constant $(0.1 \mathrm{mM})(6 \mathrm{nM}$ gold nanoparticles, $5 \mathrm{mM} \mathrm{Hg}^{2+}, 50 \mathrm{~mW}$ laser power, $100 \mathrm{~s}$ acquisition time).

gold nanoparticles exhibit no observable LSPR absorption, which is consistent with the previous reports [34,35]. The $15 \mathrm{~nm}$ and $80 \mathrm{~nm}$ gold nanoparticles show strong LSPR absorption around $520 \mathrm{~nm}$ and $553 \mathrm{~nm}$, respectively. LSPR bands of both $15 \mathrm{~nm}$ and $80 \mathrm{~nm}$ gold nanoparticles redshift with the addition of $\mathrm{Hg}^{2+}$ ions, indicating aggregation of gold nanoparticles [36-38].

Figure 3.8a shows the SERS spectra of MPA- or MBA-modified gold nanoparticles with 3 $\mathrm{nm}$ in diameter. The intensities of two $\mathrm{COO}^{-}$peaks from MPA have no observable increase upon 
addition of $\mathrm{Hg}^{2+}$. This can be reasonably explained by the EM enhancement mechanism combined with the charge transfer. $3 \mathrm{~nm}$ gold nanoparticles has no observable LSPR absorption so that EM enhancement has negligible contribution to the SERS. Also, the charge transfer cannot occur because of the unfavorable electronic structure in MPA [14]. Surprisely, all MBAmodified gold nanoparticles including the $3 \mathrm{~nm}$ gold nanoparticles without observable LSPR absorption show significant SERS enhancement. It is suggested that the charge transfer from gold to MBA induces the additional generation of SPR, which results in the additional EM contribution to the SERS. Furthermore, the enhancement in $3 \mathrm{~nm}$ gold nanoparticles is much lower than those in MBA-modified gold nanoparticles with $15 \mathrm{~nm} 80 \mathrm{~nm}$ in diameter, and that of $15 \mathrm{~nm}$ gold nanoparticles is the strongest. This is ascribed to the co-existence of EM and CE enhancements in $15 \mathrm{~nm}$ and $80 \mathrm{~nm}$ gold nanoparticles, and the LSPR position of $15 \mathrm{~nm}$ gold nanoparticles has a good overlap with the incident $532 \mathrm{~nm}$ laser. 

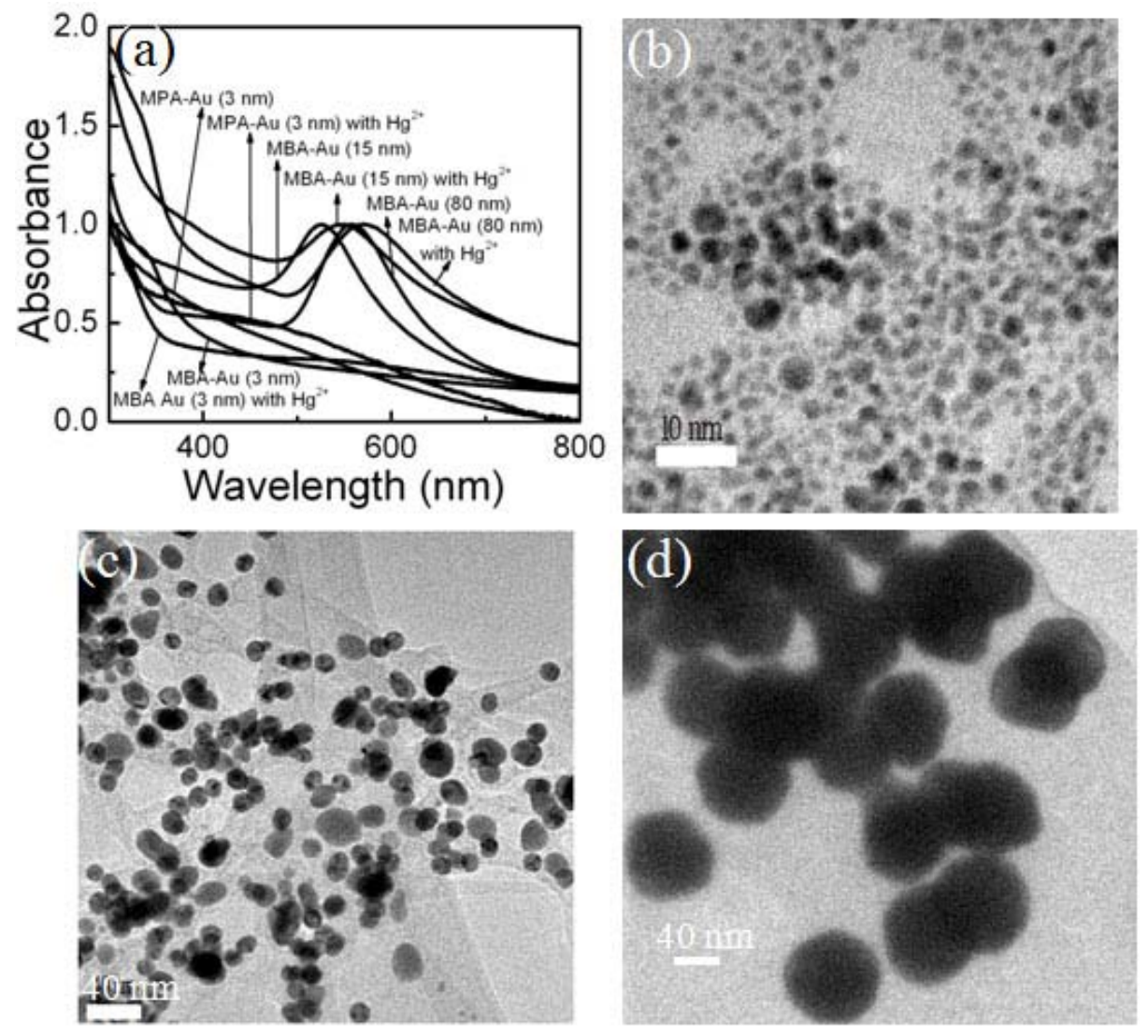

Figure 3.7 (a) UV-visible absorption spectra of $p$-mercaptobenzoic acid-modified gold nanoparticles with 3,15 and $80 \mathrm{~nm}$ in diameter in the absence and presence of $\mathrm{Hg}^{2+}$. TEM images of (b) $3 \mathrm{~nm}$, (c) $15 \mathrm{~nm}$ and (d) $80 \mathrm{~nm}$ gold nanoparticles.

Similar to that in $15 \mathrm{~nm}$ gold nanoparticles, the SERS enhancements of various TP/MPA ratios and various TP concentrations in fixed MPA concentration on $3 \mathrm{~nm}$ gold nanoparticles without observable LSPR absorption were investigated (Figure 3.8b-e). Firstly, the total concentration of TP and MPA is kept at $0.2 \mathrm{mM}$ while the TP/MPA ratio is adjusted from 0 to 8 . The peak intensity from benzene ring at $1045 \mathrm{~cm}^{-1}$ increases with the increasing TP/MPA ratio, and more importantly, the $\mathrm{COO}^{-}$peaks intensity from MPA at $881 \mathrm{~cm}^{-1}$ and $1457 \mathrm{~cm}^{-1}$ increase 

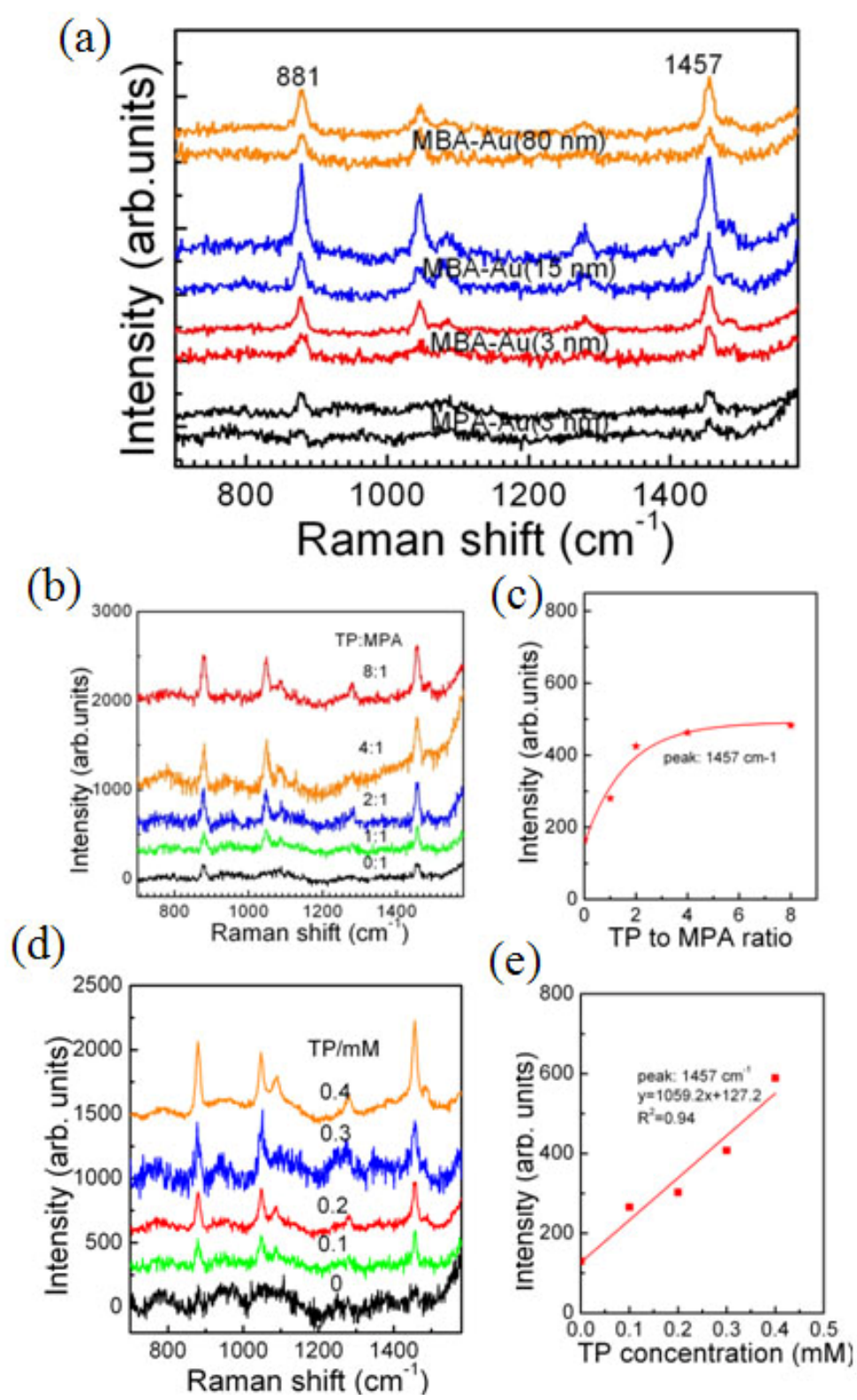

Figure 3.8 (a) SERS spectra of 3-mercaptopropionic acid- or $p$-mercaptobenzoic acid-modified gold nanoparticles with different particle sizes $(3,15$ and $80 \mathrm{~nm})$ in the absence and presence of $\mathrm{Hg}^{2+}$. (b) SERS spectra in various thiophenol/3-mercaptopropionic acid ratios adsorbed on $3 \mathrm{~nm}$ gold nanoparticles and (c) their corresponding peak intensity at $1457 \mathrm{~cm}^{-1}$ as a function of the thiophenol/3-mercaptopropionic acid ratio. (d) SERS spectra in various thiophenol 
concentrations adsorbed on $3 \mathrm{~nm}$ gold nanoparticles and (e) their corresponding peak intensity at $1457 \mathrm{~cm}^{-1}$ as a function of thiophenol concentration.

as well. Then, we checked the SERS through changing the TP amount at the fixed MPA concentration of $0.1 \mathrm{mM}$. It is found that not only are SERS signals from TP enhanced but also the SERS signals from MPA increase with the increase of TP concentration despite the MPA concentration is constant. This supports again that the charge transfer is responsible for the SERS enhancement in MPA because the LSPR is not the main reason for the SERS enhancement in 3 nm gold nanoparticles.

\subsection{Proposed operation mechanism}

Figure 3.9 schematically shows the proposed operation mechanism to explain the SERS enhancement difference in the present work. Both MBA and TP possess benzene ring of planar structure, and unique electronic level, while MPA has a linear structure. Under the incident laser, electron is excited and transferred from gold to MBA or TP while in the case of MPA, the electron transfer cannot occur due to the mismatch between Fermi level of gold and LUMO of MPA (Figure 3.9a) [39-42]. The transferred electrons can oscillate in resonance with the incident laser, similar with the generation of LSPR of gold nanoparticles. Furthermore, the transferred electrons collectively delocalize in the thin ligand layer due to the conjugated structure of MBA and TP $[43,44]$. Thus, the delocalized electrons can become the plasmon-like electrons under the laser, and result in electromagnetic enhancement of SERS. Therefore, electromagnetic contribution of charge transfer plays a dominant role in the SERS enhancement in systems containing $3 \mathrm{~nm}$ gold nanoparticles and TP or MBA molecules (Figure 3.9c). Nevertheless, there 
is negligible SERS enhancement in MPA-modified $3 \mathrm{~nm}$ gold nanoparticles because of the unable charge transfer (Figure 3.9b). If the gold nanoparticles exhibit strong LSPR absorption, both the charge transfer-induced EM enhancement and the LSPR-induced EM enhancement will contribute to the total SERS EM enhancement. This also explains why $15 \mathrm{~nm}$ and $80 \mathrm{~nm}$ gold nanoparticles show much stronger SERS enhancement in comparison with $3 \mathrm{~nm}$ gold nanoparticles (Figure 3.8).

(a)
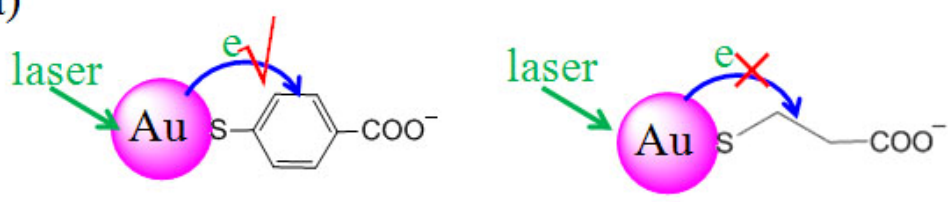

(b)

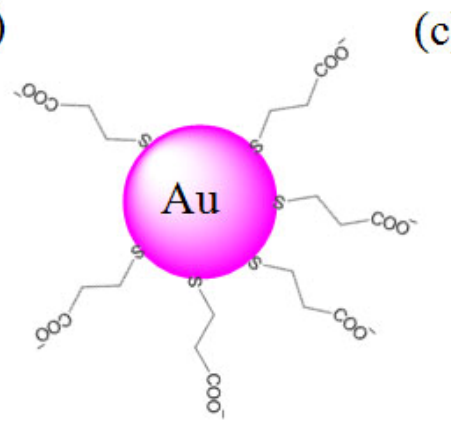

(c)

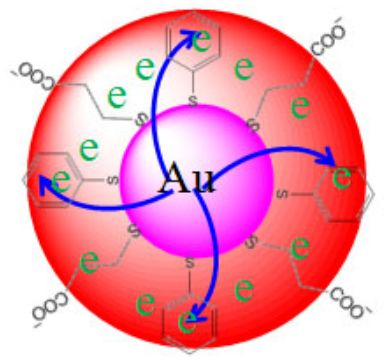

(d)

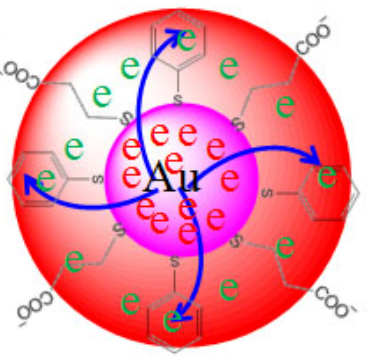

Figure 3.9 Schematic illustration of (a) charge transfer between gold nanoparticles and adsorbed molecules, proposed operation mechanism of charge transfer-induced EM enhancement (b) between $3 \mathrm{~nm}$ gold nanoparticles and 3-mercaptopropionic acid, (c) between $3 \mathrm{~nm}$ gold nanoparticles and the mixed thiophenol/3-mercaptopropionic acid, and (d) between gold nanoparticles with strong LSPR absorption and the mixed thiophenol/3-mercaptopropionic acid.

\section{9 $\mathrm{Hg}^{2+}$ concentration and $\mathrm{pH}$ value-dependent SERS}


Based on the above work, we further checked the SERS sensitivity toward the $\mathrm{Hg}^{2+}$ concentration and $\mathrm{pH}$ value (Figures 3.10 and 3.11). As mentioned above, the "hot spots" for SERS is formed by the aggregation of gold nanoparticles due to the coordination chemistry between $\mathrm{Hg}^{2+}$ and carboxylic group. On the other hand, it also can be formed by the induction of hydrogen bond between gold nanoparticles due to the terminated carboxylic group. The surface MBA molecules contain carboxylic group so that the interaction between gold nanoparticles can be adjusted upon changing the $\mathrm{pH}$ value. It can be seen that the absorbance at longer wavelength increases with the increase of $\mathrm{Hg}^{2+}$ concentration or the decrease of $\mathrm{pH}$ value. In particular, a new peak around $660 \mathrm{~nm}$ can be clearly seen in $\mathrm{pH}$ values of 1.5 and 4.7. In addition, the optical response is observed. The solution color becomes blue from red with the increased $\mathrm{Hg}^{2+}$ concentration and decreased $\mathrm{pH}$ value, and precipitates are also clearly seen at the high $\mathrm{Hg}^{2+}$ concentration and low $\mathrm{pH}$ value. Therefore, the presence of $\mathrm{Hg}^{2+}$ ion and acidic $\mathrm{pH}$ value can produce the gold nanoparticle aggregates, which is in agreement with the previous results [4548].

SERS spectra of MBA-modified gold nanoparticles with $15 \mathrm{~nm}$ in diameter were checked under different $\mathrm{Hg}^{2+}$ concentrations and $\mathrm{pH}$ values. The SERS peak intensities increase with the increase of $\mathrm{Hg}^{2+}$ concentration and the decrease of $\mathrm{pH}$ value. This is because addition of more $\mathrm{Hg}^{2+}$ and much lower $\mathrm{pH}$ values can produce more aggregates. It is worth noting that the SERS enhancement reaches saturation at $5 \mathrm{mM} \mathrm{Hg}^{2+}$ concentration, which may be due to the complete consumption of gold nanoparticles so that there is no further increase of "hot spots" even more addition of $\mathrm{Hg}^{2+}$. The present results demonstrate potential possibility of surface-enhanced Raman spectroscopy in both $\mathrm{Hg}^{2+}$ and $\mathrm{pH}$ value monitoring. However, more systematic work is 
needed to investigate and improve the performances (e.g. sensitivity, selectivity and reproducibility, etc.) of SERS sensors in these applications.

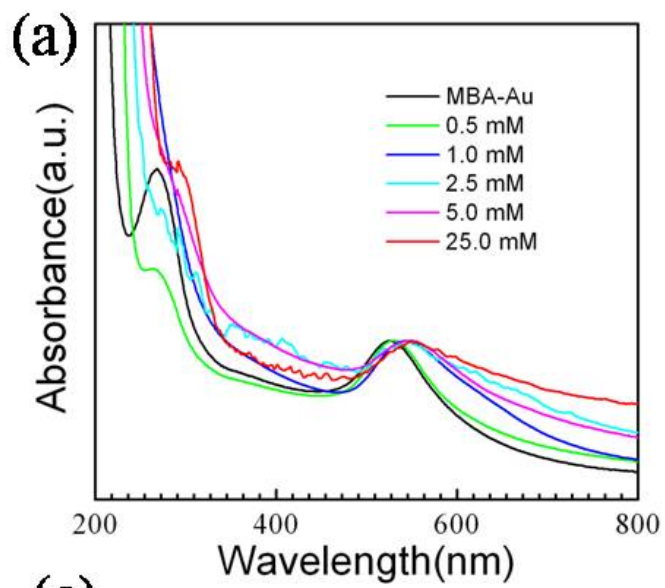

(b)
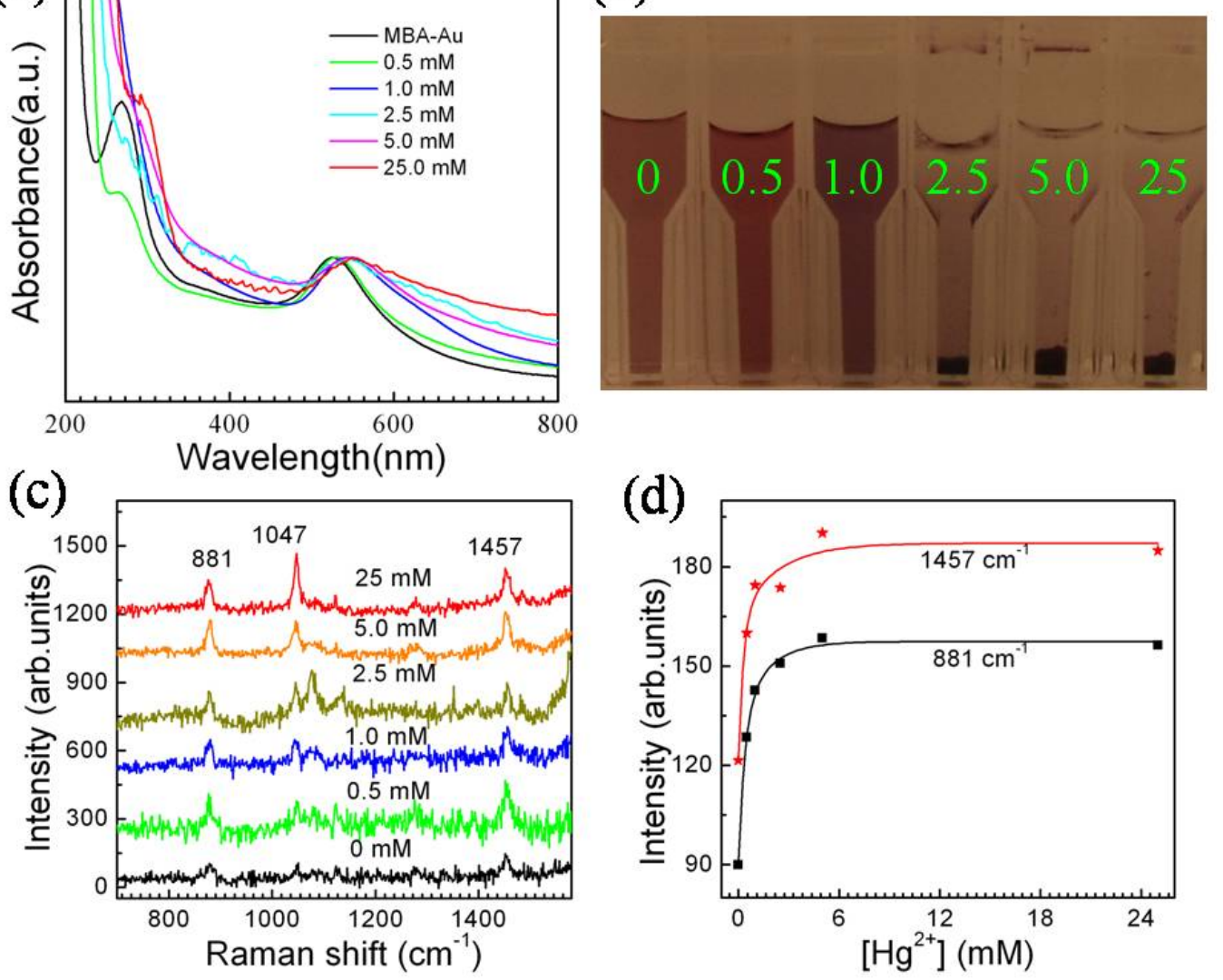

Figure 3.10 (a) UV-visible absorption spectra, (b) optical photos, (c) SERS spectra of $p$ mercaptobenzoic acid-modified gold nanoparticles $(15 \mathrm{~nm})$ in the presence of various $\mathrm{Hg}^{2+}$ concentrations, and (d) the $881 \mathrm{~cm}^{-1}$ and $1457 \mathrm{~cm}^{-1}$ peak intensities as a function of $\mathrm{Hg}^{2+}$ concentration. 


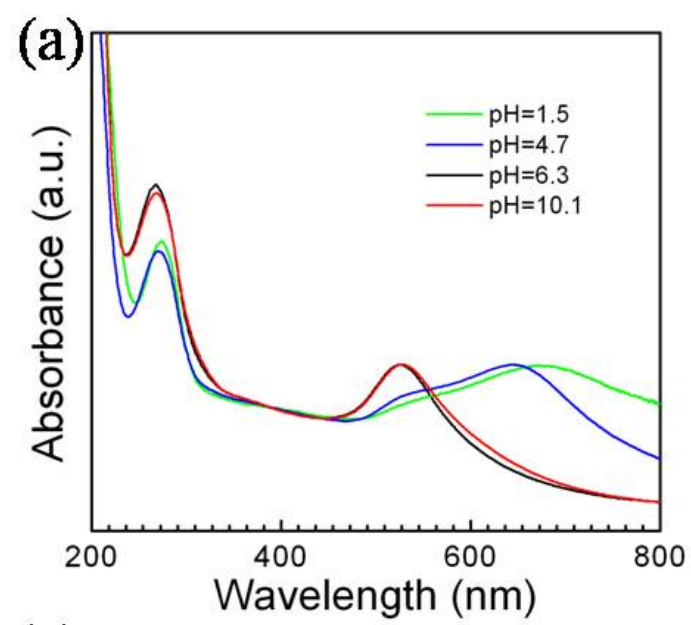

(b)
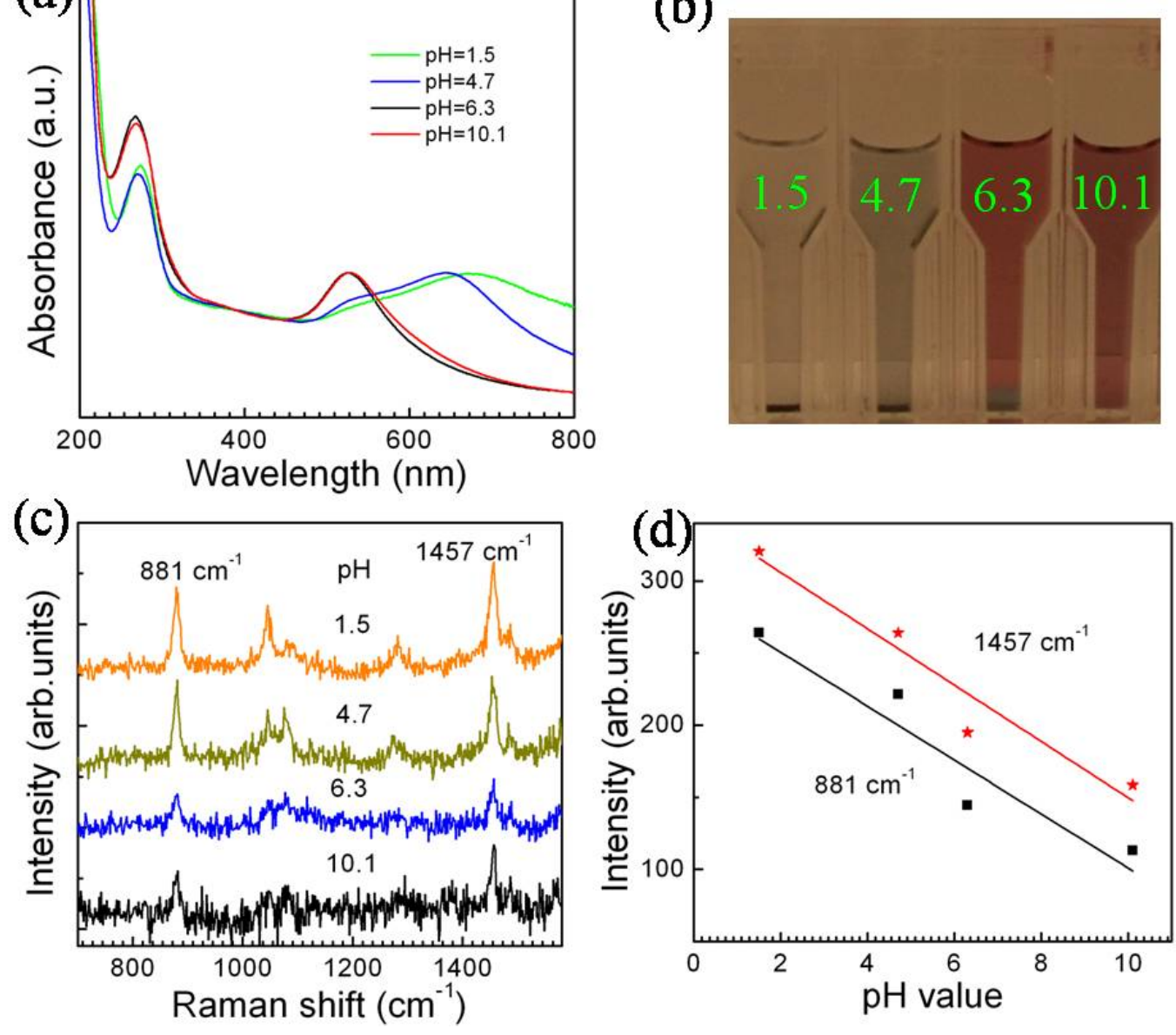

Figure 3.11 (a) UV-visible absorption spectra, (b) optical photos, (c) SERS spectra of pmercaptobenzoic acid-modified gold nanoparticles $(15 \mathrm{~nm})$ under various $\mathrm{pH}$ values, and (d) the $881 \mathrm{~cm}^{-1}$ and $1457 \mathrm{~cm}^{-1}$ peak intensities as a function of $\mathrm{pH}$ value.

\subsection{Summary}

This chapter is devoted to the role of charge transfer in the EM enhancement of SERS. Firstly, the design principle to create the "hot spots" of SERS is introduced. TP, MBA, MPA and Cys, which bear different molecular structures, are chosen for the Raman tag molecules, and the 
divalent ions (e.g. $\mathrm{Hg}^{2+}$ ) are used to induce the aggregation through the coordination chemistry. Following this, the difference of SERS activity among these molecules was checked on $15 \mathrm{~nm}$ gold nanoparticles. The MBA with benzene ring exhibits the strongest SERS than the linear MPA and Cys. Then, the SERS was investigated through changing the TP/MPA ratio at a fixed total concentration, and changing the TP amount at a fixed MPA concentration. It was found that more addition of TP increases not only the Raman peak intensity of benzene ring around 1045 $\mathrm{cm}^{-1}$ but also the $\mathrm{COO}^{-}$peak intensity from MPA. Based the present experimental results, we propose transferred electrons from gold to MBA or TP can delocalize in the whole molecule layer, and thus induce the electromagnetic enhancement of surface Raman scattering.

In order to confirm the proposed mechanism, we carried out the further investigation on different sized gold nanoparticles. $15 \mathrm{~nm}$ and $80 \mathrm{~nm}$ gold nanoparticles with strong LSPR absorption show stronger SERS activity in comparison with $3 \mathrm{~nm}$ gold nanoparticles. $3 \mathrm{~nm}$ gold nanoparticles still have SERS activity even it has weak LSPR absorption, which supports the above claims that the charge transfer is responsible for the difference of SERS activities. In the following work, systematic investigation was carried out on $3 \mathrm{~nm}$ gold nanoparticles, similar with that in $15 \mathrm{~nm}$ gold nanoparticles. The proposed charge transfer-induced EM enhancement was demonstrated. Finally, $\mathrm{Hg}^{2+}$ concentration and $\mathrm{pH}$ value-dependent SERS were investigated, which shows the promising applications of SERS in $\mathrm{Hg}^{2+}$ and $\mathrm{pH}$ value monitoring.

\section{References}

[1] Sekhar, P. K.; Brosha, E. L.; Mukundan, R.; Garzon, F. H. Electrochem. Soc. Interf., 2010, $35-40$.

[2] Graham, D. Angew. Chem. Int. Ed., 2010, 49, 9325 - 9327. 
[3] Nie, S.; Emory, S. R. Science, 1997, 275, 1102-1106;

[4] Park, W.-H.; Kim, Z. H. Nano Lett., 2010, 10, 4040-4048.

[5] Cao, Y. W. C.; Jin, R.; Mirkin, C. A. Science, 2002, 297, 1536-1539.

[6] Li, J. F.; Huang, Y. F.; Ding, Y.; Yang, Z. L.; Li, S. B.; Zhou, X. S.; Fan, F. R.; Zhang, W.; Zhou, Z. Y.; Wu, D. Y.; Ren, B.; Wang, Z. L.; Tian, Z. Q. Nature, 2010, 464, 392-395.

[7] Campion, A.; Kambhampati, P. Chem. Soc. Rev., 1998, 27, 241-250.

[8] Zhang, J.; Li, X.; Sun, X.; Li, Y. J. Phys. Chem. B, 2005, 109, 12544-12548.

[9] Dadosh, T.; Sperling, J.; Bryant, G. W.; Breslow, R.; Shegai, T.; Dyshel, M.; Haran, G.; BarJoseph, I. ACS Nano, 2009, 3, 1988-1994.

[10] Haynes, C. L.; Van Duyne, R. P. J. Phys. Chem. B, 2003, 107, 7426-7433.

[11] Yu, X.; Cai, H.; Zhang, W.; Li, X.; Pan, N.; Luo, Y.; Wang, X.; Hou, J. G. ACS Nano, 2011, $5,952-958$.

[12] Blackie, E. J.; Le Ru, E. C.; Etchegoin, P. G. J. Am. Chem. Soc., 2009, 131, 14466-14472.

[13] Morton, S. M.; Jensen, L. J. Am. Chem. Soc., 2009, 131, 4090-4098.

[14] Tao, N. J. Nat. Nanotech., 2006, 1, 173-181.

[15] Kinnan, M. K.; Chumanov, G. J. Phys. Chem. C, 2007, 111, 18010-18017.

[16] Basu, S.; Pande, S.; Jana, S.; Bolisetty, S.; Pal, T. J. Am. Chem. Soc., 2008, 24, 5562-5568.

[17] Li. D.; Wieckowska, A.; Willner, I. Angew. Chem. Int. Ed., 2008, 47, 3927-3931.

[18] Ojea-Jiménez, I.; Puntes, V. J. Am. Chem. Soc., 2009, 131, 13320-13327.

[19] Huang, C. C.; Chiang, C. K.; Lin, Z. H.; Lee, K. H.; Chang, H. T. Anal. Chem., 2008, 80, 1497-1504.

[20] Chen, S.; Kimura, K. Langmuir, 1999, 15, 1075-1082. 
[21] Rodriguez, J. A.; Dvorak, J.; Jirsak, T.; Liu, G.; Hrbek, J.; Aray, Y.; González, C. J. Am. Chem. Soc., 2003, 125, 276-285.

[22] Pensa, E.; Carro, P.; Rubert, A. A.; Benítez, G.; Vericat, C.; Salvarezza, R. C. Langmuir, 2010, 26, 17068-17074.

[23] Lee, S. J.; Moskovits, M. Nano Lett., 2011, 11, 145-150.

[24] Kim, Y.; Johnson, R. C.; Hupp, J. T. Nano Lett., 2001, 1, 165-167.

[25] Zamborini, F. P.; Leopold, M. C.; Hicks, J. F.; Kulesza, P. J.; Malik, M. A.; Murray, R. W. J. Am. Chem. Soc., 2002, 124, 8958-8964.

[26] Socrates, G. Infrared and Raman Characteristic Group Frequencies, Tables and Charts, $3^{\text {rd }}$ ed.; John Wiley\& Sons, Ltd.: New York, 2001.

[27] Kumar, C. S. S. R.; Mohammad, F. J. Phys. Chem. Lett., 2010, 1, 3141-3146.

[28] Xue, X.; Wang, F.; Liu, X. J. Am. Chem. Soc., 2008, 130, 3244-3245.

[29] Zhou, Y.; Wang, S.; Zhang, K.; Jiang, X. Angew. Chem. Int. Ed., 2008, 47, 7454-7456.

[30] Fleger, Y.; Mastai, Y.; Rosenbluh, M.; Dressler, D. H. Surf. Sci., 2009, 603,788-793.

[31] Michota, A.; Bukowska, J. J. Raman Spectrosc., 2003, 34, 21-25.

[32] Chen, G.; Wang, Y.; Yang, M.; Xu, J.; Goh, S. J.; Pan, M.; Chen, H. J. Am. Chem. Soc., 2010, 132, 3644-3645.

[33] Li, W.; Camargo, P. H. C.; Lu, X.; Xia, Y. Nano Lett., 2009, 9, 485-490.

[34] Templeton, A. C.; Chen, S.; Gross, S. M.; Murray, R. W. Langmuir, 1999, 15, 66-76.

[35] Kim, J.; Lee, D. J. Am. Chem. Soc., 2007, 129, 7706-7707.

[36] Volkert, A. A.; Subramaniam, V.; Ivanov, Michael R.; Goodman, A. M.; Haes, A. J. ACS Nano, 2011, 5, 4570-4580. 
[37] Zhang, J.; Wang, L.; Pan, D.; Song, S.; Boey, F. Y. C.; Zhang, H.; Fan, C. small, 2008, 4, $1196-1200$.

[38] Yang, J.; Lee, J. Y.; Too, H.-P.; Chow, G.-M.; Gan, L. M. Chem. Phys., 2006, 323, 304312.

[39] Reimers, J. R.; Wang, Y.; Cankurtaran, B. O.; Ford, M. J. J. Am. Chem. Soc., 2010, 132, $8378-8384$.

[40] Nagata, C.; Yamaguchi, T. Radiat. Res., 1978, 73, 430-439.

[41] Özcan, M.; Karadagg, F.; Dehrii, I. Acta Phys. -Chim. Sin., 2008, 24, 1387-1392.

[42] Maul, R.; Preuss, M.; Ortmann, F.; Hannewald, K.; Bechstedt, F. J. Phys. Chem. A, 2007, $111,4370-4377$.

[43] Karzazi, Y.; Crispin, X.; Kwon, O.; Brédas, J. L.; Cornil, J. Chem. Phys. Lett., 2004, 387, $502-508$.

[44] Kim, B.; Beebe, J. M.; Jun, Y.; Zhu, X.-Y.; Frisbie, C. D. J. Am. Chem. Soc., 2006, 128, 4970-4971.

[45] Slocik, J. M.; Zabinski, Jr., J. S.; Phillips, D. M.; Naik, R. R. small, 2008, 4, 548-551.

[46] Jiang, Y.; Zhao, H.; Lin, Y.; Zhu, N.; Ma, Y.; Mao, L. Angew. Chem. Int. Ed., 2010, 49, $4800-4804$.

[47] Guan, J.; Jiang, L.; Li, J.; Yang, W. J. Phys. Chem. C, 2008, 112, 3267-3271.

[48] Nam, J.; Won, N.; Jin, H.; Chung, H.; Kim, S. J. Am. Chem. Soc., 2009, 131, 13639-13645. 


\section{CHAPTER 4: SURFACE-ENHANCED RAMAN SCATTERING IN VARIOUS SHAPED GOLD NANOSTRUCTURES}

\subsection{Introduction}

A surface-enhanced Raman scattering (SERS) device typically consists of an incident laser, detection set-up, SERS substrate and the Raman signal molecule (also called as Raman tag, Raman label or Raman reporter). In general, laser and detection set-up are commercially available so that they will not be inclued in the present work. As discussed in Chapter 3, Raman tag is an important component for high quality SERS device, and will influence the SERS not only through the enhancement mechanism but also the other ways, e.g. background [1-3]. In addition, the SERS substrate is of fundamental importance for the SERS, because the electromagnetic field for the SERS is produced by the silver or gold substrate according to the dominant electromagnetic enhancement (EM) [4-6], and the SERS substrate is necessary for the charge transfer process as well [7].

Recently, we have witnessed an increased interest in the development of SERS for applications in biochemistry, chemical production and environmental monitoring because of its molecular fingerprinting information and its potential of multiplexing detection [8-12]. Currently, it is widely accepted that the SERS enhancement was attributed to the strong EM and the chemical enhancement (CE) with enhancement factors (EFs) of $10^{4}-10^{12}$ and $10-10^{2}$, respectively[4-7,13]. The CE mechanism is mainly due to an electronic resonance charge transfer process between metal and molecule adsorbed on the metal surface $[7,13,14]$, while the EM 
mechanism originates from collective oscillations of the conduction electrons in the metal, called the localized surface plasmon excitation (LSPR) [15]. A large number of investigations show that the EM enhancement is much more efficient than the CE enhancement. The energies (frequency) of LSPR depend on the particle size, shape, composition, the orientation of the electric field relative to the particle as well as the dielectric property of the surrounding medium [16-19]. Correlation between LSPR and SERS has been conducted by the classical Mie theory in previous work. There is no doubt that a combination of theory with experiments improves our understanding for EM mechanism in SERS [16,18].

Persistent experimental and theoretical efforts are made to investigate the size-, shape- and composition-dependent LSPR properties of gold and silver nanoparticles [17,20-22]. The particle size and substrate composition can affect the LSPR position, and the electromagnetic field distribution is dependent on the geometric architecture of the materials [23,24]. In addition, concentration of electromagnetic field was found in the anisotropic nanostructures, espectially sharp tips in triangles, elongated nanorods, nanowires or nanostars, which is expected to generate the "hot spots" and amplify SERS signals in an effective manner [25]. Thus, excellent substrates for SERS applications can be foreseen for nanomaterials that posseses sharp tips with large density within its structure. However, limited attentions have been paid to the size- and shapedependent SERS studies and their relationship with LSPR even the shape of nanostructure has been demonstrated to be a key factor for the LSPR distribution $[17,26]$.

As is well-known, chemical sensing and biological imaging are two of important applications of SERS, which require nanomaterials biocompatible, nontoxic and stable in biological environments. Most of the currently available synthetic methods provide SERS devices through direct adsorption of Raman tag onto the nanomaterial surface, and the Raman tag is consequently 
in direct contact with the surrounding environment $[27,28]$. In these cases, the Raman tag usually desorbs from the surface, which leads to fluctuation and poor reproducibility of Raman signal. SERS substrates with high sensitivity and excellent reproducibility are extremely desirable to realize effective sensing. A feasible strategy to obtain a stable and reproducible Raman-tagged SERS substrate is to encapsulate the Raman tag in a protective shell such as $\mathrm{SiO}_{2}$ and polymer $[29,30]$. In particular, $\mathrm{SiO}_{2}$ is of interest as a protective layer because of its long-term stability against degradation and easy functionalization with a wide range of functional groups.

In this chapter, we compared the SERS activity of gold nanospheres, nanorods and nanostars under resonance and nonresonance excitation (532 $\mathrm{nm}$ and $785 \mathrm{~nm}$ ), and the three-dimentional finite-difference time domain (FDTD) method was used to investigate the physical origin of the shape-dependent SERS through correlating the SERS enhancement factor with the electromagnetic field distribution. Furthermore, $\mathrm{SiO}_{2}$ encapsulation of the malachite green isothiocyanate (MGITC)-tagged gold nanospheres, nanorods and nanostars was carried out, and the stability of Raman signal and its application in DNA hybridization monitoring were demonstrated.

\subsection{Materials and experimental methods}

\subsubsection{Materials and instruments}

Chloroauric acid trihydrate $\left(\mathrm{HAuCl}_{4} \cdot 3 \mathrm{H}_{2} \mathrm{O}\right)$, trisodium citrate dehydrate $\left(\mathrm{Na}_{3} \mathrm{C}_{6} \mathrm{H}_{5} \mathrm{O}_{7} \cdot 2 \mathrm{H}_{2} \mathrm{O}\right.$, ACS, 90.0+\%), $\mathrm{Na}_{2} \mathrm{HPO}_{4}(99.0 \%), \mathrm{NaH}_{2} \mathrm{PO}_{4}(99.0 \%)$ and sodium hydroxide were purchased from Alfa Aesar. Malachite green isothiocyanate (MGITC) was purchased from Molecular Probes. 3-mercaptopropyltrimethoxylsilane (MPTMS) and 3-triethoxylsilylpropyl succinic 
anhydride (TEPSA) and (3-aminopropyl) trimethoxylsilane (APTMS) were purchased from Gelest Inc. N-hydroxysuccinimide (NHS), 1-ethyl-3-(3-dimethylaminopropyl)-carbodiimide (EDC), $5 \mathrm{M} \mathrm{NaCl}$ solution (BioUltra) and sodium silicate stock solution $\left(26.5 \% \mathrm{SiO}_{2}\right.$ in $10.6 \%$ $\mathrm{Na}_{2} \mathrm{O}$ ) were purchased from Sigma-Aldrich. Oligonucleotides (DNAs) were obtained from Integrated DNA Technologies (IDT). Deionized (D.I.) water produced by a Milli-Q Millipore system (18.2 $\mathrm{M} \Omega / \mathrm{cm}^{2}$, Millipore Corp., USA) was used for the preparation of all the solutions. All solvents were obtained from the commercial sources and used without further purification.

TEM images were taken on a JEM $2100 \mathrm{~F}$ transmission electron microscope with an acceleration voltage of $200 \mathrm{kV}$. UV-visible absorption spectra were measured in a range of 200$900 \mathrm{~nm}$ on a Shimadzu UV-2550 spectrometer. Raman spectra were collected on a Renishaw inVia Raman spectrometer at an excitation laser of $532 \mathrm{~nm}$. A microscope equipped with $20 \times$ objective was used to focus the incident excitation laser. The laser power on the sample was 50 mW. Raman spectra were also obtained in a handheld Raman spectrometer (Inspector Series, DeltaNu) at an excitation laser of $785 \mathrm{~nm}$. For Raman measurement, a drop of solution was placed onto the glass slide. Calibration and normalization of the Raman spectra were done with Si or polystyrene. Three spectra from different sites were collected from each sample, and averaged to represent the SERS results.

\subsubsection{Synthesis of gold nanospheres, nanorods and nanostars}

Gold nanospheres that were about $15 \mathrm{~nm}$ in diameter was synthesized using a trisodium citrate reduction method described in the literature [31]. Briefly, trisodium citrate $(2 \mathrm{~mL}, 1 \mathrm{wt} \%)$ was added to a boiling aqueous solution of $\mathrm{HAuCl}_{4} \cdot 3 \mathrm{H}_{2} \mathrm{O}(50 \mathrm{~mL}, 0.4 \mathrm{mM})$, and the solution was kept continually boiling for $30 \mathrm{~min}$ to give a wine-red solution. After cooled down to room temperature, the solution was stored in a $4{ }^{\circ} \mathrm{C}$ refrigerator. The concentration of the prepared 
gold nanospheres was calculated to be $12 \mathrm{nM}$ by the UV-vis absorption spectroscopy based on the Beer-Lambert Law using the molar extinction coefficient of $1 \times 10^{8} \mathrm{M}^{-1} \cdot \mathrm{cm}^{-1}[32]$.

Gold nanorods and nanostars were synthesized according to the well-documented seedmediated growth method $[33,34]$. Specifically, for the synthesis of seeds for the gold nanorods, CTAB aqueous solution $(5 \mathrm{~mL}, 0.20 \mathrm{M})$ was mixed with $5.0 \mathrm{~mL}$ of $0.5 \mathrm{mM} \mathrm{HAuCl}_{4}$. Then 0.60 $\mathrm{mL}$ of ice-cold $10 \mathrm{mM} \mathrm{NaBH}_{4}$ solution was added. After vigorous stirring, the seed solution was kept at $25{ }^{\circ} \mathrm{C}$. For the nanorod growth, the growth solution was prepared separately. CTAB (5 $\mathrm{mL}, 0.20 \mathrm{M}$ ) was first added to $0.15 \mathrm{~mL}$ of $4 \mathrm{mM} \mathrm{AgNO}_{3}$ solution at $25{ }^{\circ} \mathrm{C}$, followed by $5.0 \mathrm{~mL}$ of $1 \mathrm{mM} \mathrm{HAuCl}_{4}$ and $70 \mu \mathrm{L}$ of $78.8 \mathrm{mM}$ ascorbic acid. The final step was the addition of $12 \mu \mathrm{L}$ of the seed solution to the growth solution at $27-30{ }^{\circ} \mathrm{C}$. The temperature of the growth medium was kept constant at $27-30{ }^{\circ} \mathrm{C}$ in all preparations. The reaction was allowed to last for about 10 h.

To synthesize gold nanostars, $\mathrm{HAuCl}_{4} \cdot 3 \mathrm{H}_{2} \mathrm{O}$ aqueous solution $(1 \mathrm{~mL}, 1 \mathrm{wt} \%)$ was first diluted by water $(90 \mathrm{~mL})$, followed by the injection of sodium citrate $(2 \mathrm{~mL}, 38.8 \mathrm{mM})$. Subsequently, freshly prepared $\mathrm{NaBH}_{4}$ solution $(1 \mathrm{~mL}, 0.075 \mathrm{wt} \%$ in $38.8 \mathrm{mM}$ sodium citrate solution) was added and then the reaction mixture was stirred overnight at room temperature to form the seed solution. Subsequently, $50 \mathrm{~mL}$ of gold seed solution was mixed with PVP (10 $\mathrm{mM}$ ) for $24 \mathrm{~h}$ at room temperature. After that, $82 \mu \mathrm{L}$ of $50 \mathrm{mM} \mathrm{HAuCl} \mathrm{H}_{4}$ aqueous solution was mixed with $15 \mathrm{~mL}$ of $10 \mathrm{mM}$ PVP in DMF, followed by the rapid addition of $43 \mu \mathrm{L}$ of the PVPcoated gold seed solution $([\mathrm{Au}] \approx 4 \mathrm{mM})$ under stirring at room temperature. The reaction was allowed to last for about $13 \mathrm{~h}$.

The as-prepared gold nanorod and gold nanostar solutions were centrifuged twice and redispersed in D.I. water for further preparations. 


\subsection{3 $\mathrm{SiO}_{2}$ encapsulation of MGITC-tagged gold nanoparticles}

Gold nanoparticles (nanospheres, nanorods or nanostars) tagged by MGITC and encapsulated by a thin $\mathrm{SiO}_{2}$ layer, called gold nanosphere, nanorod or nanostar@MGITC@ $\mathrm{SiO}_{2}$, were synthesized according to previously published methods with a slight modification [35-37]. As for gold nanosphere@MGITC@ $\mathrm{SiO}_{2}, 50 \mu \mathrm{L}$ MGITC aqueous solution $(100 \mu \mathrm{M})$ was added to a 20 $\mathrm{mL}$ citrate-stabilized gold nanosphere solution, and kept stirring for $30 \mathrm{~min}$. $200 \mu \mathrm{L}$ fresh APTMS aqueous solution $(2.0 \mathrm{mM})$ was added, and the solution was stirred for another $30 \mathrm{~min}$. $1 \mathrm{~mL}$ fresh sodium silicate solution $(0.54 \mathrm{wt} \%, \mathrm{pH}>12)$ was added, and stirred for $10 \mathrm{~min}$. Then, $20 \mathrm{~mL}$ ethanol was added after standing for one day to generate a condensed $\mathrm{SiO}_{2}$ layer, and the solution stood for one more day. Finally, the resulting solution was centrifuged at a speed of $13,300 \mathrm{rpm}$ to get a red precipitate, and washed at least 5 times using ethanol and D.I. water to remove impurities, respectively.

We used a similar method to synthesize gold nanorod@MGITC@SiO $\mathrm{Si}_{2}$ and gold nanostar@MGITC@SiO $\mathrm{Si}_{2}$ with a replacement of APTMS by MPTMS because MPTMS has much stronger affinity toward gold surface than APTMS. The as-prepared gold nanorods and nanostars were initially coated by a high density layer of surfactants from the synthetic process so that MPTMS is difficult to be adsorbed onto the gold nanorod and nanostar surface. Therefore, for $\mathrm{SiO}_{2}$ coating, gold nanorod and nanostar were first purified by centrifugation and washing with ethanol, and then redispersed into D.I. water. The absorbance of gold nanorod and nanostar was adjusted to 1.35 and 2.79 at the long wavelenght LSPR peaks. $2.5 \mu \mathrm{L}$ MGITC solution was added to $1 \mathrm{~mL}$ purified gold nanorod or nanostar solution, and then stirred for 30 min. $50 \mu \mathrm{L}$ fresh MPTMS solution $(50 \mathrm{mM})$ was added and continue to stir for another $30 \mathrm{~min}$. Then, $100 \mu \mathrm{L}$ fresh sodium silicate solution $(0.54 \mathrm{wt} \%, \mathrm{pH}>12)$ was added, and stirred for 10 
min. $1 \mathrm{~mL}$ ethanol was added after standing for one day to generate a condensed $\mathrm{SiO}_{2}$ layer, and the solution stood for one more day. The resulting solution was centrifuged and washed by ethanol. The precipitates were redispersed into the D.I. water for further use.

\subsubsection{DNA functionalization and its hybridization monitoring}

$\mathrm{SiO}_{2}$ slides were first successively cleaned by sonication with $\mathrm{CH}_{2} \mathrm{Cl}_{2}, \mathrm{CH}_{3} \mathrm{OH}$ and D.I. water for $10 \mathrm{~min}$, respectively, and then heated at $90{ }^{\circ} \mathrm{C}$ in a $20 \mathrm{~mL}$ solution containing $\mathrm{NH}_{4} \mathrm{OH}$ and peroxide $\left(\mathrm{NH}_{4} \mathrm{OH}: \mathrm{H}_{2} \mathrm{O}_{2}: \mathrm{H}_{2} \mathrm{O}=1: 1: 5\right)$ for $1 \mathrm{~h}$. $\mathrm{SiO}_{2}$ slides were sequentially washed with ethanol and D.I. water, and dried at $60{ }^{\circ} \mathrm{C}$ for $1 \mathrm{~h}$ in a vacuum oven. The cleaned $\mathrm{SiO}_{2}$ slides were incubated overnight in a $20 \mathrm{~mL}$ ethanolic solution of $200 \mathrm{mM}$ TEPSA, and then washed using ethanol and D.I. water. The $\mathrm{COOH}$ groups on $\mathrm{SiO}_{2}$ silde were activated by a solution containing $50 \mathrm{mM}$ NHS and $200 \mathrm{mM}$ EDC in the $0.3 \mathrm{PBS}$ solution $\left(10 \mathrm{mM} \mathrm{Na} 2 \mathrm{HPO}_{4} / \mathrm{NaH}_{2} \mathrm{PO}_{4}, 0.3 \mathrm{M}\right.$ $\mathrm{NaCl}, \mathrm{pH}=7.0)$, and then $50 \mu \mathrm{L} 0.1 \mathrm{mM} 5^{\prime}$-amine-modified single-strand DNA (5'- $\mathrm{NH}_{2}-\left(\mathrm{CH}_{2}\right)_{6}{ }^{-}$ TCT AGC AGT TAC G-3') in a PBS solution was added onto activated TEPSA-modified $\mathrm{SiO}_{2}$ slides, and incubated for at least $24 \mathrm{~h}$. After immobilization of single-strand DNA, the $\mathrm{SiO}_{2}$ slide was rinsed using D.I. water and PBS solution, respestively.

2 mL gold nanostar@MGITC@SiO $\mathrm{SiO}_{2}$ was centrifuged and washed using D.I. water, and redispersed into $1 \mathrm{~mL}$ TEPSA ethanolic solution $(200 \mathrm{mM})$. After standing for $24 \mathrm{~h}$, the solution was centrifuged and washed using D.I. water and PBS, respectively. The resulting solids were TEPSA-modified gold nanostar@MGITC@ $\mathrm{SiO}_{2}$, and redispersed into $800 \mu \mathrm{L}$ PBS solution. The COOH groups of TEPSA-modified gold nanostar@MGITC@ $\mathrm{SiO}_{2}$ were activated by adding 100 $\mu \mathrm{L}$ solution containing $50 \mathrm{mM}$ NHS and $200 \mathrm{mM}$ EDC, and then $50 \mu \mathrm{L}$ of two DNA sequences (3'-A GAT CGT CAA TGC- $\left(\mathrm{CH}_{2}\right)_{6}-\mathrm{NH}_{2}-5^{\prime}$ ' or 3'-A GCC GGA ATT CAG- $\left(\mathrm{CH}_{2}\right)_{6}-\mathrm{NH}_{2}-5^{\prime}, 0.1$ $\mathrm{mM}$ ) dissolved in PBS solution were added to $500 \mu \mathrm{L}$ activated TEPSA-modified gold 
nanostar@MGITC@SiO ${ }_{2}$, respectively. After incubated for 24 h, the solution was centrifuged and washed using PBS solution and redispersed into $800 \mu \mathrm{L}$ PBS solution for further use.

As for the DNA hybridization, $50 \mu \mathrm{L}$ of DNA-functionalized gold nanostar@MGITC@ $\mathrm{SiO}_{2}$ PBS solution were added to the DNA-functionalized $\mathrm{SiO}_{2}$ slides. After 30 min incubation, the $\mathrm{SiO}_{2}$ slides were rinsed using D.I. water, and used for further measurement.

\subsubsection{Finite difference time domain simulations}

The open source software MEEP was used for the three-dimensional FDTD simulations. An explanation of the implementation of the FDTD algorithm and PML layers in MEEP can be found in this reference [38]. The shapes were created inside of a computation cell where the surrounding dielectric was air. The dielectric function used was a series of four Lorentz sums fit to the data of Johnson and Christy [39] The fit was optimized at about $520 \mathrm{~nm}$ to ensure that the nanosphere's peak position agreed with the values predicted by Mie theory. A plane wave, constant wavelength source (at either 532 or $785 \mathrm{~nm}$ ) was utilized. The remaining boundary conditions were perfectly matched layers. The electromagnetic field was output at all points in a volume containing the shape. The total EM enhancement was performed by dividing the electromagnetic field on the surface of the shape by the incident electromagnetic field in the absence of the shape. The experimental and simulated absorption spectra agreed for all of the shapes.

\subsection{Morphorlogy and absorption spectra of gold nanoparticles}

The TEM images showed the size and morphology of three types of gold nanoparticles (Figure 4.1). The nanospheres were $15 \mathrm{~nm}$ in a diameter while the nanorods were $30.2 \mathrm{~nm}$ long 
and $9.3 \mathrm{~nm}$ in a diameter with an aspect ratio of 3.2. The nanostars showed a core of $34.5 \mathrm{~nm}$ in diameter. Figure 4.2 shows the UV-Visible absorption spectra of the nanospheres, nanorods and nanostars. The nanospheres showed a characteristic LSPR absorption at $520 \mathrm{~nm}$. The nanorods exhibited the transverse and longitudinal plasmon bands at $515 \mathrm{~nm}$ and $735 \mathrm{~nm}$, respectively. The nanostars displayed two LSPR bands at $532 \mathrm{~nm}$ and $675 \mathrm{~nm}$.
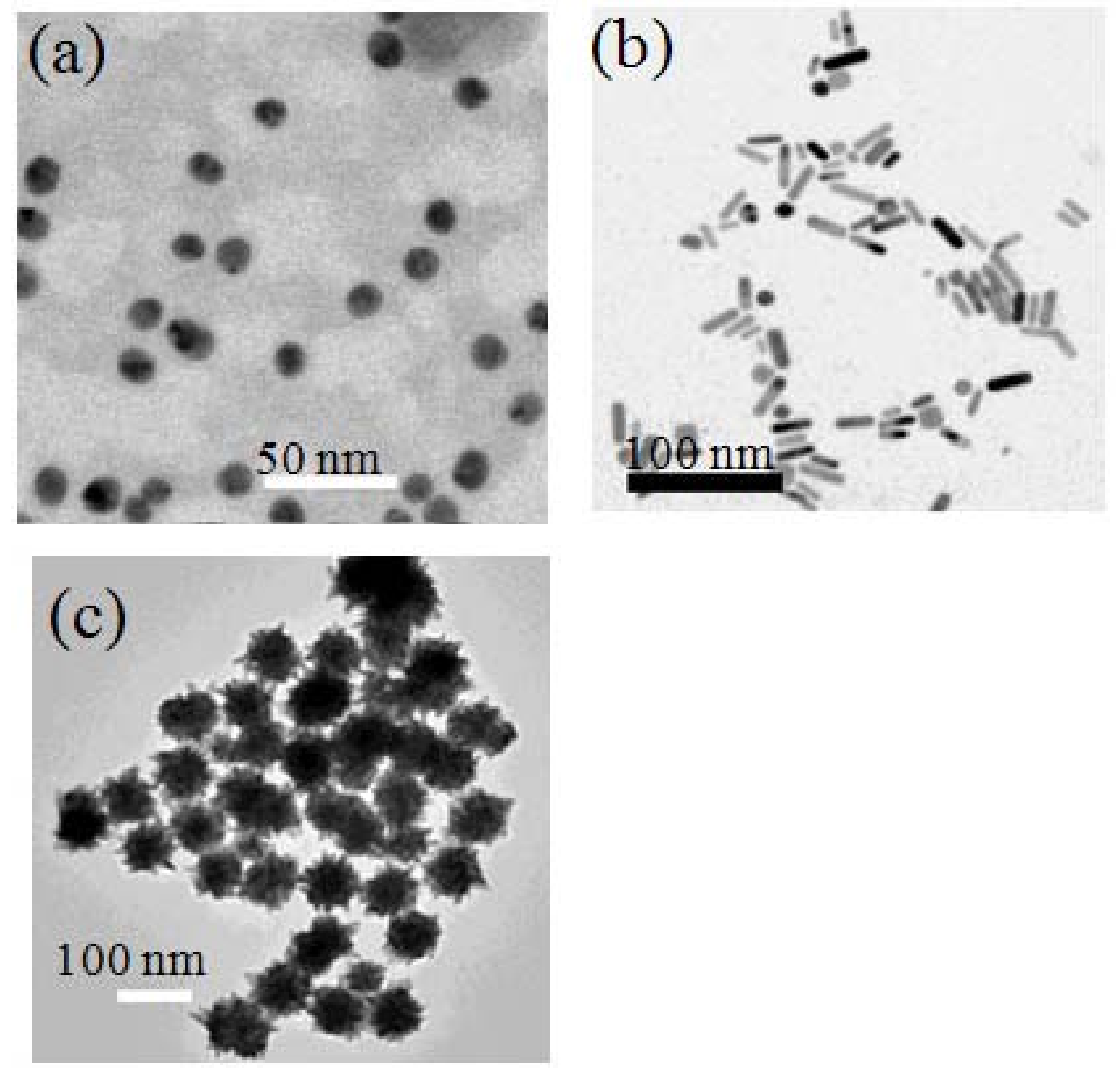

Figure 4.1 TEM images of as-made gold nanospheres, gold nanorods and gold nanostars. 


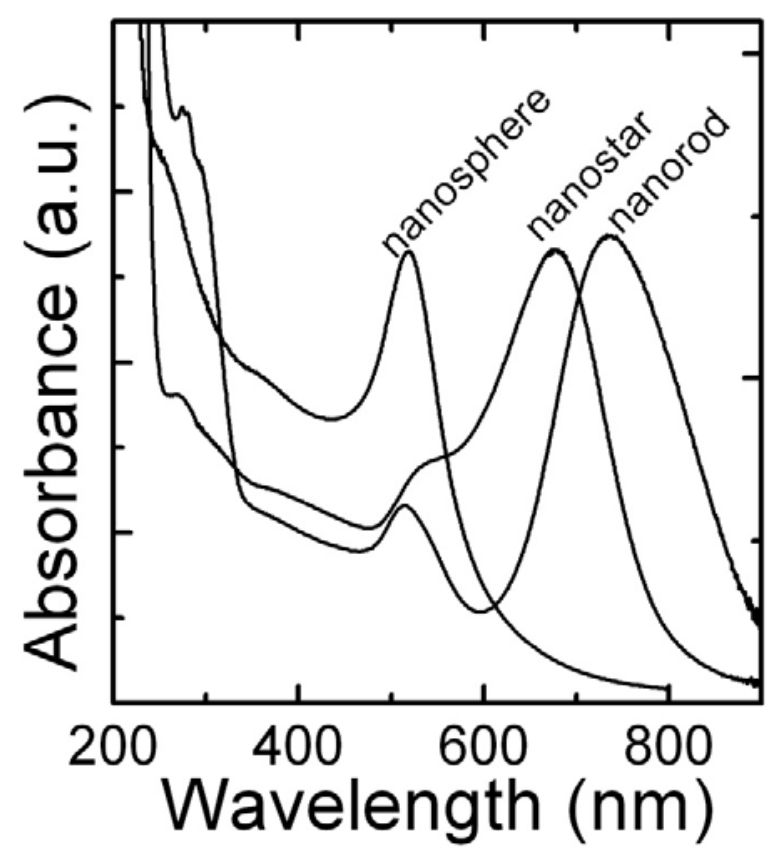

Figure 4.2 UV-visible absorption spectra of gold nanospheres, gold nanorods and gold nanostars.

\subsection{Shape-dependent SERS in gold nanoparticles}

We first established which among the nanosphere, nanorod and nanostar produced the strongest SERS enhancement when MGITC was attached to the gold nanoparticles. Figure 4.3 shows the SERS spectra of MGITC in the aqueous solutions containing nanospheres, nanorods and nanostars at a concentration of $6 \mathrm{nM}$ under exciation of the $532 \mathrm{~nm}$ and $785 \mathrm{~nm}$ lasers, respectively. Such a low nanoparticle concentration was chosen in order to avoid the aggregation of nanomaterials so that the contribution of aggregation to the SERS can be minimized to an insignificant level. In addition, the resonance Raman enhancement was excluded in the present system because both $532 \mathrm{~nm}$ and $785 \mathrm{~nm}$ lasers did not match with the optical absorption of MGITC [40]. Another rationale is that isothiocyanate $(-\mathrm{N}=\mathrm{C}=\mathrm{S})$ group in MGITC can be bound 
to the gold surface and compatible with the $\mathrm{SiO}_{2}$ encapsulation process $[9,41]$. Table 4.1 lists the assignments of selected Raman peaks of MGITC in SERS spectra $[42,43]$.

(a)

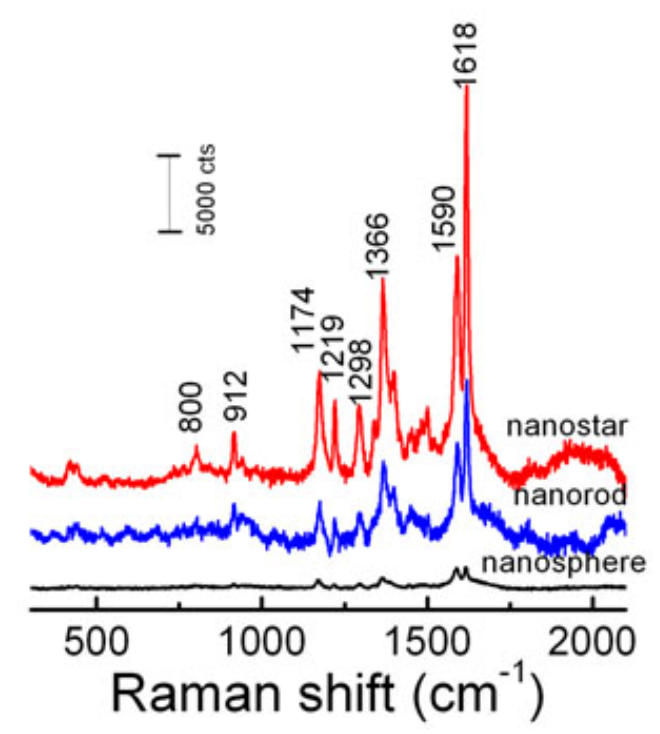

(b)

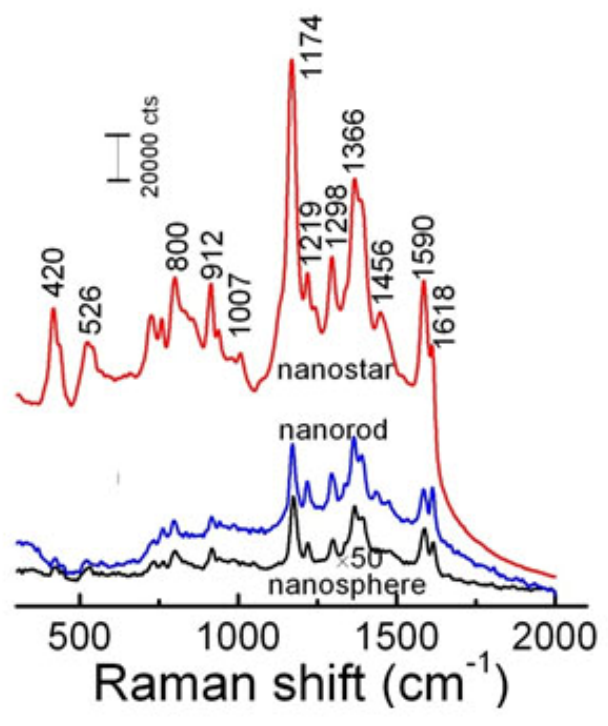

Figure 4.3 SERS spectra of MGITC $\left(2.5 \times 10^{-7} \mathrm{M}\right)$ in $6 \mathrm{nM}$ gold nanospheres, nanorods and nanostars at (a) $532 \mathrm{~nm}$ laser (laser power: $50 \mathrm{~mW}$, integration time: $10 \mathrm{~s}$ ) and (b) $785 \mathrm{~nm}$ laser (laser power: $120 \mathrm{~mW}$, integration time: $10 \mathrm{~s}$ ).

The SERS spectra are similar to those reported in previous work (Figure 4.3) $[41,44]$. It is obviously seen that gold nanostar has the highest SERS activity under both $532 \mathrm{~nm}$ and $785 \mathrm{~nm}$ lasers, and gold nanorod has much higher SERS activity than that in the nanosphere under both $532 \mathrm{~nm}$ and $785 \mathrm{~nm}$. Based on the $1366 \mathrm{~cm}^{-1}$ peak, the experimental SERS enhancement factor $(\mathrm{EF})$ is calculated as $[3,45]$

$$
\mathrm{EF}=\frac{I_{\mathrm{SERS}}}{I_{\mathrm{b}}} \cdot \frac{C_{\mathrm{b}}}{C_{\mathrm{SERS}}}
$$


Table 4.1 Assignments of selected Raman bands of MGITC in SERS spectra $[42,43]$

\begin{tabular}{cl}
\hline Peak position $\left(\mathrm{cm}^{-1}\right)$ & \multicolumn{1}{c}{ Assignment } \\
1618 & Phenyl-N and C-C stretching \\
1590 & In-plane ring stretching and bending \\
1456 & $\mathrm{~N}\left(\mathrm{CH}_{3}\right)_{2}$ bending and rocking \\
1366 & Aromatic ring stretching \\
1298 & In-plane C-H and C-C-H bending \\
1219 & N-C stretching mode \\
1174 & In-plane C-H bending \\
1007 & In-plane benzene E $1 v$ \\
912 & B 1 in plane benzene ring \\
800 & Out-of-plane C-H bending \\
526 & In-plane benzene ring \\
420 & Out-of-plane benzene ring \\
\hline
\end{tabular}

where $I_{\mathrm{SERS}}$ and $I_{\mathrm{b}}$ are the respective Raman intensities at $1366 \mathrm{~cm}^{-1}$ in the presence and absence of gold nanoparticles, and $\mathrm{C}_{\mathrm{SERS}}$ and $\mathrm{C}_{\mathrm{b}}$ are the molar concentrations of Raman tag in the presence and absence of gold nanoparticles. Table 4.2 lists the experimental EFs of three types of gold nanoparticles under excitation of the $532 \mathrm{~nm}$ and $785 \mathrm{~nm}$ lasers. The incident $532 \mathrm{~nm}$ laser was in resonance with the LSPR band $(520 \mathrm{~nm})$ of the nanospheres. The $532 \mathrm{~nm}$ laser was also resonant with the transverse LSPR band of the nanorods and one of the two LSPR bands (532 $\mathrm{nm}$ ) of the nanostars. The $785 \mathrm{~nm}$ laser was in resonance with the primary LSPR bands of both the nanostars and nanorods but not with the nanospheres. However, gold nanospheres showed the lowest EFs while gold nanostars showed the highest EFs under both lasers of $532 \mathrm{~nm}$ and 
$785 \mathrm{~nm}$. It can be seen from Table 4.2 that the EFs of the nanostars were 1-3 orders of magnitude higher than those of the nanospheres and the nanorods under excitation of the laser of the same wavelength (either $532 \mathrm{~nm}$ or $785 \mathrm{~nm}$ laser). Therefore, it can be inferred from the above results that the SERS enhancement is strongly dependent on the geometric shape of nanoparticles.

Table 4.2 Experimental and calculated average SERS enhancement factors.

\begin{tabular}{llllll}
\hline Sample & \multicolumn{2}{c}{ Experimental EF } & & \multicolumn{2}{c}{ Calculated EF } \\
\cline { 2 - 3 } \cline { 5 - 6 } & $532 \mathrm{~nm}$ laser & $785 \mathrm{~nm}$ laser & & $532 \mathrm{~nm}$ laser & $785 \mathrm{~nm}$ laser \\
\hline Gold nanosphere & $5.1 \times 10^{2}$ & $3.8 \times 10^{2}$ & & $8.7 \times 10^{2}$ & $8.3 \times 10^{2}$ \\
Gold nanorod & $1.6 \times 10^{3}$ & $5.1 \times 10^{4}$ & & $1.5 \times 10^{3}$ & $1.6 \times 10^{4}$ \\
Gold nanostar & $3.9 \times 10^{3}$ & $1.0 \times 10^{5}$ & & $3.0 \times 10^{3}$ & $8.9 \times 10^{4}$ \\
\hline
\end{tabular}

\subsection{Electric field distribution and theoretical SERS enhancement factor}

The LSPR peaks depend on the nanoparticle size, shape, and composition as well as on the orientation of the electromagnetic field relative to the particle and the dielectric property of the surrounding medium [16-19]. The LSPR induces a strong local electromagnetic field surrounding the surface of the nanoparticles [15], which results in the electromagnetic enhancement of Raman signals. In the present work, the three-dimensional FDTD method was employed to simulate the LSPR-induced electromagnetic field of the three types of gold nanoparticles under both $532 \mathrm{~nm}$ and $785 \mathrm{~nm}$ incident lasers (Figure 4.4).The results showed that the electromagnetic fields around the nanoparticles were more intense and dropped down fast with distance away from the particle. The nanostars showed the highest calculated value of the maximum electric field intensity under both the excitations. The nanorods exhibited lower maximum electric field intensity under the $532 \mathrm{~nm}$ laser, and much higher maximum electric 
field intensity under the $785 \mathrm{~nm}$ laser when the field was aligned with longitudinal mode of the nanorod, which may be due to favorable orientation of incident laser for the longitudinal LSPR mode and the strong longitudinal LSPR absorption. Furthermore, it should be noted that the maximum electric field intensity appeared around the sharp tip in the anisotropic structure, in agreement with the previous reports showing that the electromagnetic field can be concentrated at the sharp tip of nanostructures $[24,25,46]$. The confined LSPR at these sharp tips generated "hot spots" and resulted in the dominant contribution to the SERS intensity. As the SERS signal obtained from experiment is an averaged signal over the entire three-dimensional space, we calculated the average EF by integrating the total electromagnetic field over the surface of each shape and normalizing it by the incident electromagnetic field over the same area in the absence of the shape according to the following equation:[3,47]

$$
E F_{\text {cal }}=\int_{A}\left|\frac{\mathrm{E}_{\text {loc }}\left(\omega_{0}\right)}{\mathrm{E}_{0}\left(\omega_{0}\right)}\right|^{4} d A
$$

where $E_{0}$ is the incident electromagnetic field, $E_{\text {loc }}$ is the local electromagnetic field at the surface, $\omega_{0}$ is the incident frequency and $A$ is the surface area of nanoparticles. Although the absolute values differ, the simulated SERS EFs followed a similar order as the experimental results (Table 4.2) in both $532 \mathrm{~nm}$ and $785 \mathrm{~nm}$ lasers. Since the simulated results are normalized by the surface area, they reflect the EF of all adsorbed SERS active molecules and the combined measurement signal. In order to maximize the EF, the LSPR field must have high peak values and be distributed across the surface. The nanospheres have a dipole mode which is relatively weak and diffusely spread across the surface as seen in Figure 4.4. This leads to a lower enhancement in both the experimental and theoretical values as seen in Table 4.2 Both nanostars and nanorods show the highest EF at $785 \mathrm{~nm}$ where the stronger LSPR mode exists, which create 
a higher localized electromagnetic field leading to one order of magnitude enhancement in comparison with the nanosphere. The nanostar has the highest EF because the LSPR is concentrated into every projection on its surface. The nanostar shows the highest localized electromagnetic field and does this over the largest surface area making it the most effective at enhancing SERS as seen in the experimental values.

The difference between experimental EFs and simulated EFs mainly evolves from the imperfections of the simulated shapes in reproducing the actual experiment. Several experimental factors affect the measured SERS enhancement. The synthesized samples do not have a perfect size distribution. The difference in sizes will contribute to experimental error within each sample. However, the simulations support that the geometric features of a shape but not its size has a more important effect on the EF enhancement. Since the SERS active molecules are adsorbed on the surface of the particle, it is important to maximize the localized electromagnetic field enhancement that exists outside of the volume of the nanoparticle. The simulations showed that this was achieved with sharp geometric features which concentrate the LSPR in a lightning rod effect. These sharp features can account for order of magnitude differences in the totalized electromagnetic field enhancement. The simulated electromagnetic field enhancements were normalized by the surface area in the calculation, eliminating differences in the total electromagnetic enhancement that could arise purely from size differences. The agreement between the calculated and experimental values showed that the geometric features are the most important variables in determining the SERS enhancement. 

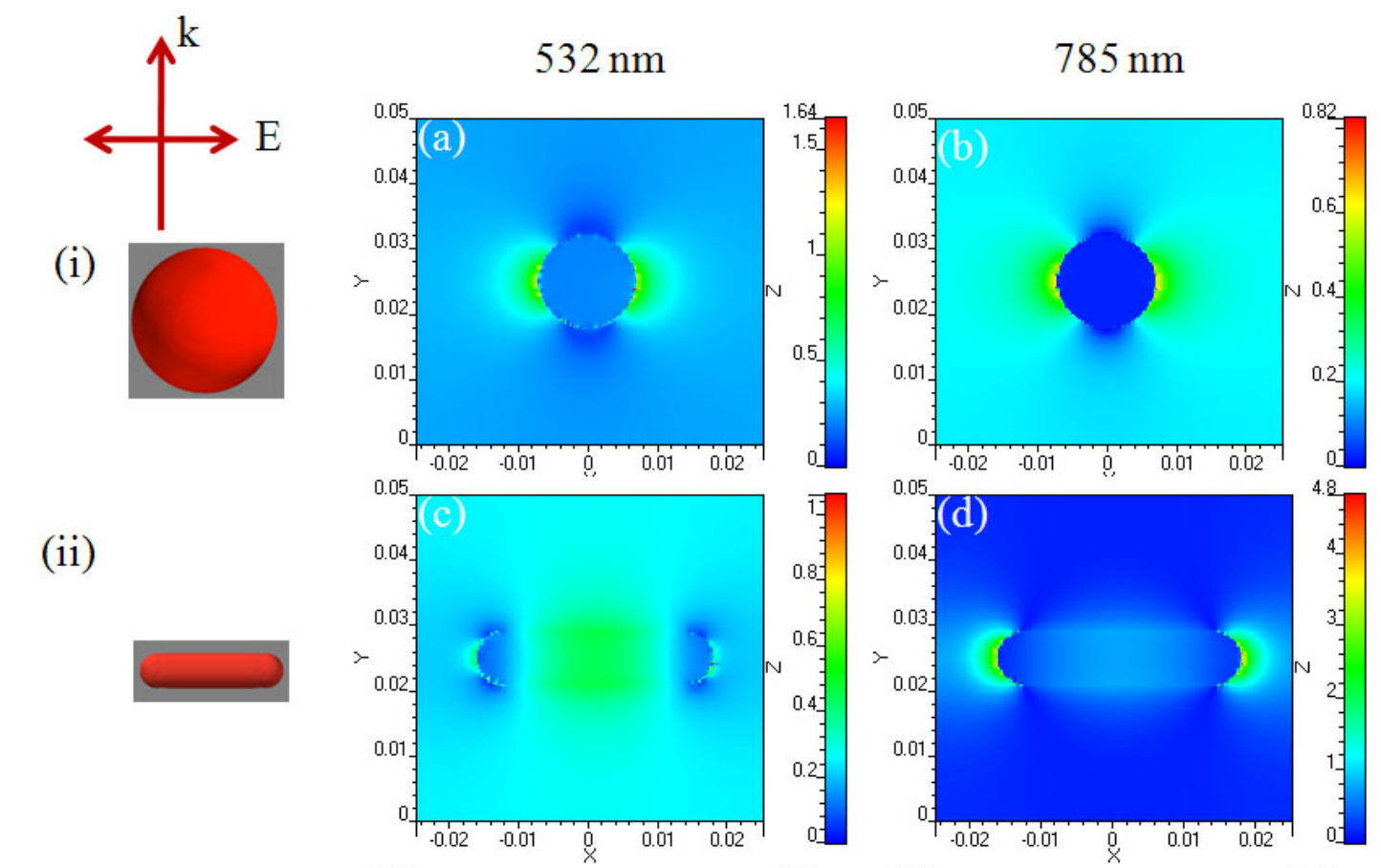

(iii)
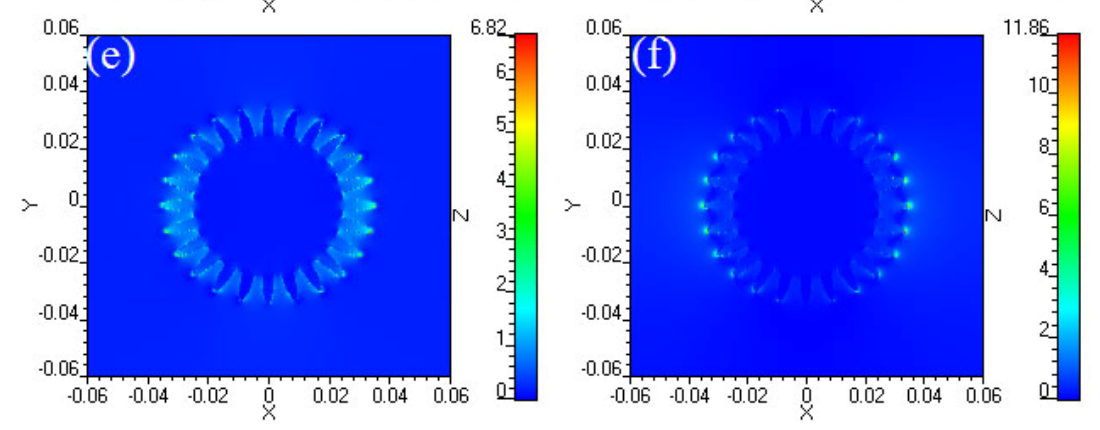

Figure 4.4 3D-FDTD simulated electromagnetic field distributions of a gold i) nanosphere, ii) nanorod and iii) nanostar under excitations of (a,c,e) $532 \mathrm{~nm}$ and (b,d,f) $785 \mathrm{~nm}$ lasers. The intensity scale is normalized as $\mathrm{E} / \mathrm{E}_{0}$.

\section{6 $\mathrm{SiO}_{2}$ encapsulation of MGITC-tagged gold nanoparticles}

Most of present studies in SERS directly attached the Raman tag onto the nanomaterial surface [48-54]. One of disadvatages is that the Raman tag in these systems suffers desorption 
from the nanomaterial surface due to the exposure to the robust environment, which leads to poor reproducibility and stability of SERS signal in practical applications [29]. Herein, we encapsulated MGTIC-tagged gold nanoparticles by $\mathrm{SiO}_{2}$ to avoid the fluctuation of Raman signal over time and environment. Figure 4.5 schematically describes synthesis of the gold@MGITC@SiO 2 . We first attached the non-fluorescent organic molecule, MGITC, onto gold nanospheres, nanorods or nanostars, and then coated a very thin $\mathrm{SiO}_{2}$ pretective layer. APTMS or MPTMS was used to modify the gold surface because the terminal amine/thiol has a strong affinity for gold and the active functional ethoxyl group was exposed to facillitate the adsorption of sodium silicate. Unlike tetraethylorthosilicate (TEOS) commonly used in the conventional silica coating method, the addition of sodium silicate can result in a very thin $\mathrm{SiO}_{2}$ layer. It must be pointed out that gold nanorods and nanostars need to be purified by centrifugation before $\mathrm{SiO}_{2}$ coating to remove the high density surfactants, because the strong binding and high density of surfactants from the synthetic procedure could block the access of ATPMS or MPTMS to the gold surface [35-37]. 


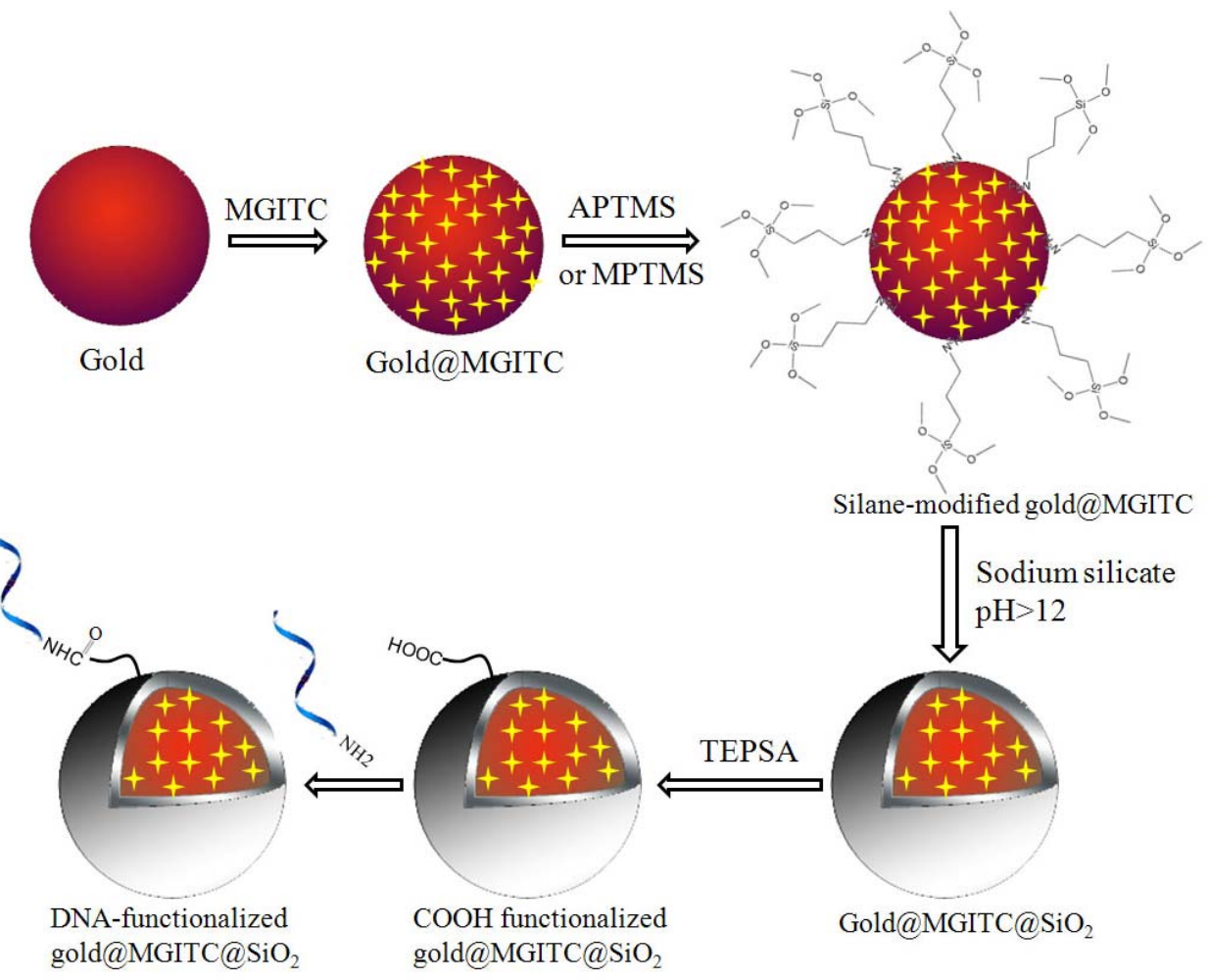

Figure 4.5 Schematical illustration of preparation of gold@MGITC@ $\mathrm{SiO}_{2}$ and its DNA functionalization.

Figure 4.6 and Figure 4.7 show the UV-visible absorption spectra and TEM images of gold nanosphere, nanorod and nanostar@MGITC@SiO $\mathrm{Si}_{2}$. TEM images demonstrate the successful $\mathrm{SiO}_{2}$ coating of gold nanospheres, nanorods and nanostars with a 3-5 $\mathrm{nm} \mathrm{SiO} \mathrm{S}_{2}$ layer in which no significant change of LSPR is observed. 


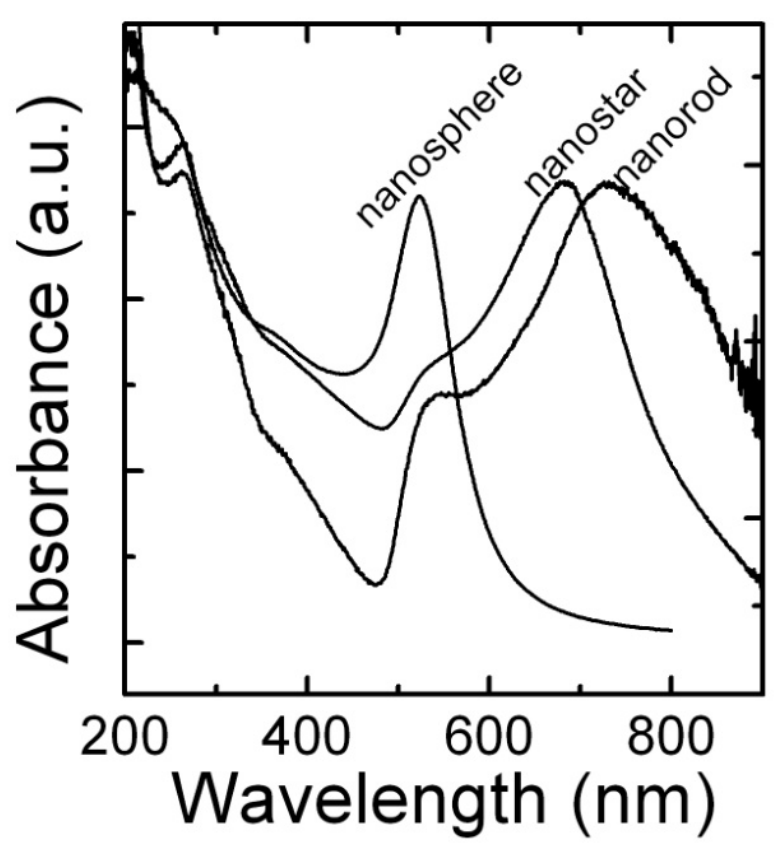

Figure 4.6 UV-visible absorption spectra of gold nanosphere, nanorod and nanostar@MGITC@SiO

\subsection{SERS of gold@MGITC@SiO $\mathrm{SiO}_{2}$ composite particles}

The SERS activities of all materials were examined under two incident lasers of $532 \mathrm{~nm}$ and $785 \mathrm{~nm}$, respectively. All samples show very strong SERS signal as shown in Figure 4.8. The intensity difference may be due to the different MGITC amounts on the surface of gold nanospheres, nanorods and nanostars. The $\mathrm{SiO}_{2}$ encapsulation prevents the Raman tag from leaking to the outside and is expected to render excellent long-term stability and signal reproducibility in practical applications. We also checked the SERS of the solid gold@MGITC@SiO 2 , and they exhibit a similar SERS spectra except the different intensities. 

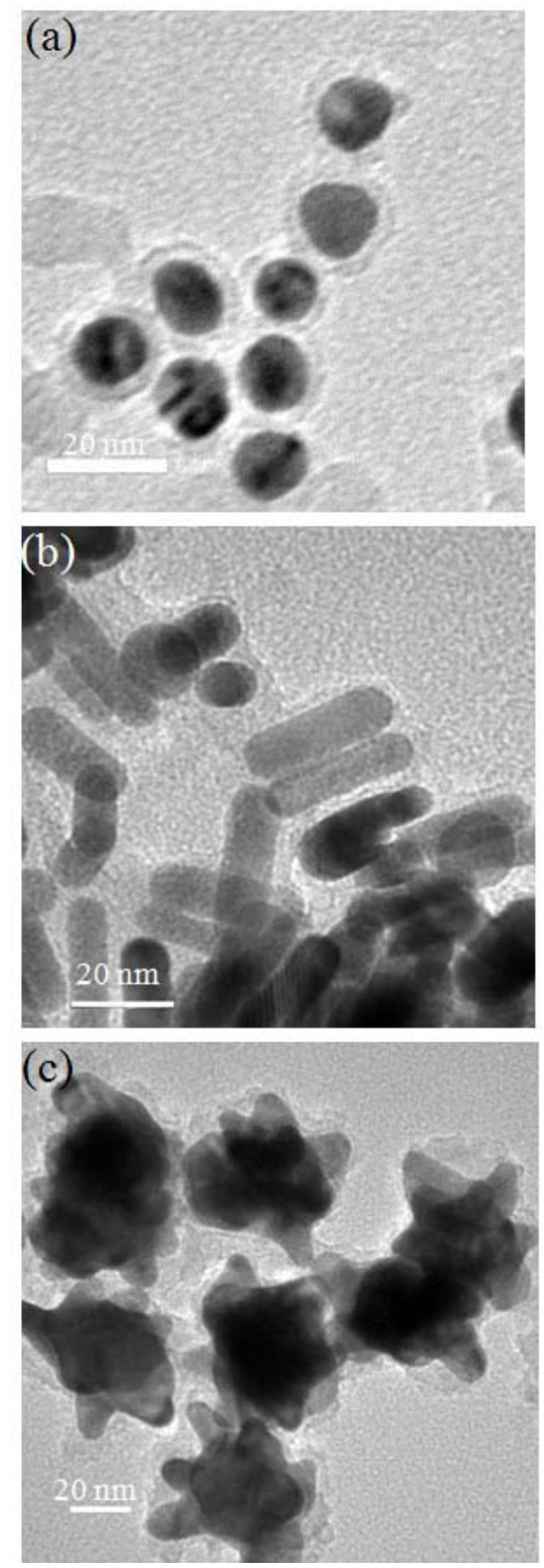

Figure 4.7 TEM images of (a) gold nanosphere@MGITC@ $\mathrm{SiO}_{2}$, (b) gold nanorod@MGITC@SiO ${ }_{2}$ and (c) gold nanostar@MGITC@SiO 

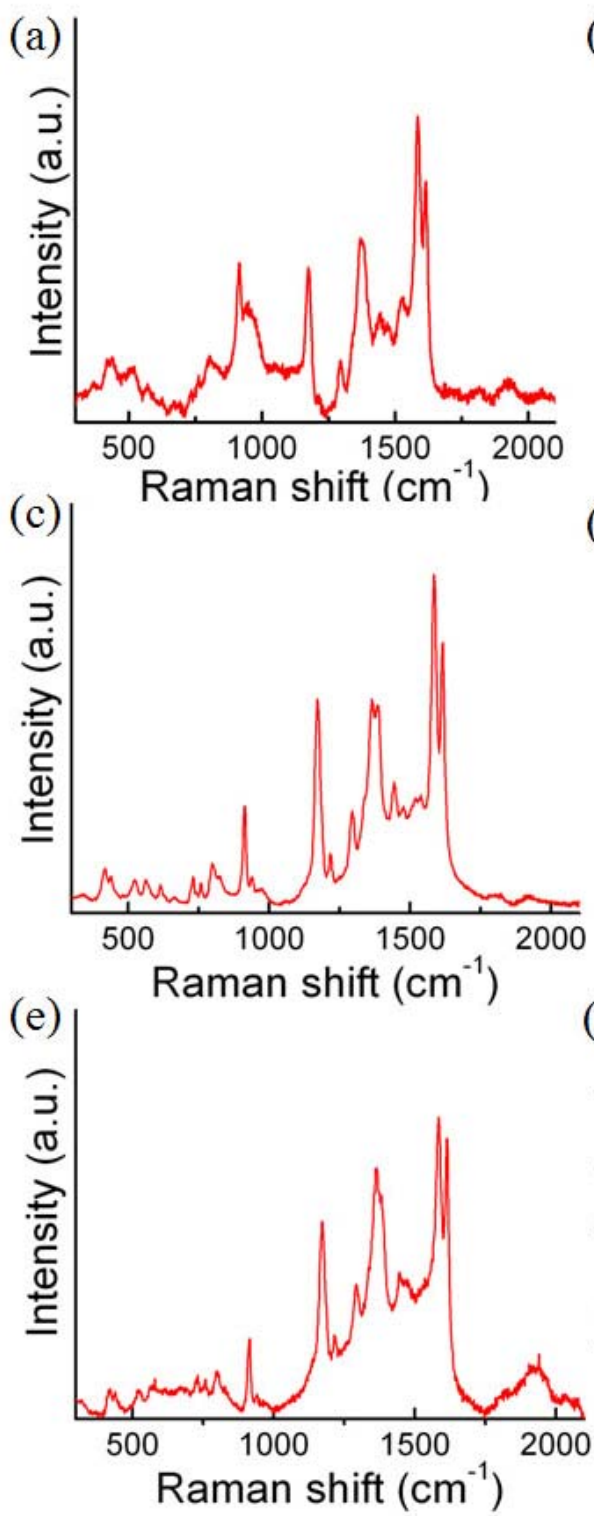
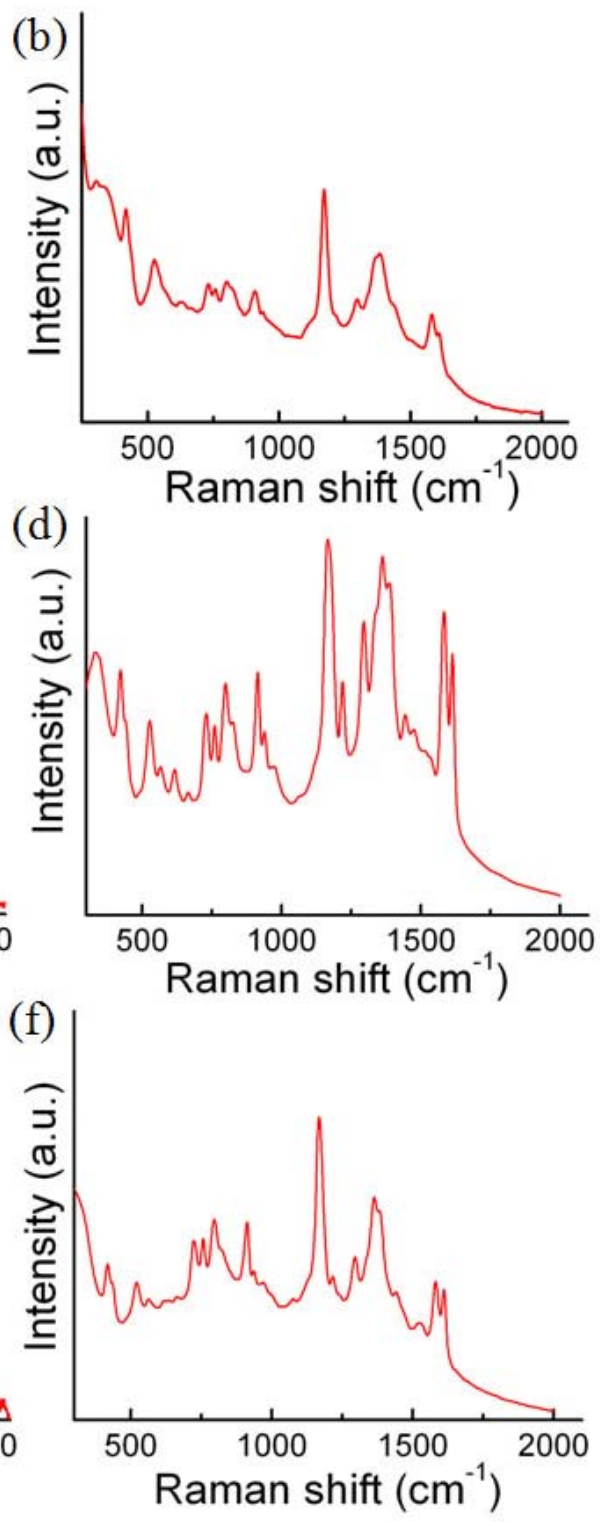

Figure 4.8 Raman spectra of gold nanosphere@MGITC@ $\mathrm{SiO}_{2}$ under (a) 532 nm and (b) 785 nm, gold nanorod@MGITC@ $\mathrm{SiO}_{2}$ under (c) 532 nm and (d) 785 nm lasers, and gold nanostar@MGITC@SiO $\mathrm{Sind}_{2}$ under (e) 532 nm and (f) 785 nm lasers. 


\subsection{Effects of solvents and salt concentrations on stability}

As mentioned above, the uncoated gold nanoparticlces including nanospheres, nanorods and nanostares investigated here will aggregate and then the LSPR band disappears or red-shifts. For the visual color response of gold nanospheres, we investigated effects of representive solvents and salt concentrations on the stability of gold nanosphere without and with $\mathrm{SiO}_{2}$ coating (Figure 4.9). The result indicates that uncoated gold nanosphere is stable in D.I. water or PBS solution but quickly aggregate in ethanol, 2-propanol or in PBS with $0.3 \mathrm{M} \mathrm{NaCl}(0.3 \mathrm{M} \mathrm{PBS})$, which is agreement with the previous reports [40]. UV-visible absorption spectra show the LSPR band at $520 \mathrm{~nm}$ is retained in D.I. water or PBS while a new band around $672 \mathrm{~nm}$ can be clearly seen in ethanol, 2-propanol or 0.3 M PBS. Furthermore, it can be seen that the gold nanosphere@MGITC@ $\mathrm{SiO}_{2}$ exhibits excellent color response stability in solvents and $\mathrm{NaCl}$ concentrations investigated, and the LSPR band position keeps constant under these environments. It should be noted that gold nanosphere@MGITC@ $\mathrm{SiO}_{2}$ has a poor dissolvability in 2-propanol but the LSPR band still is kept at $520 \mathrm{~nm}$. Therefore, the present result demonstrates that $\mathrm{SiO}_{2}$ encapsulation of gold nanoparticles improves the LSPR stability in robust environments and should have promising applications in biology, chemistry and environmental monitoring. 
(a)
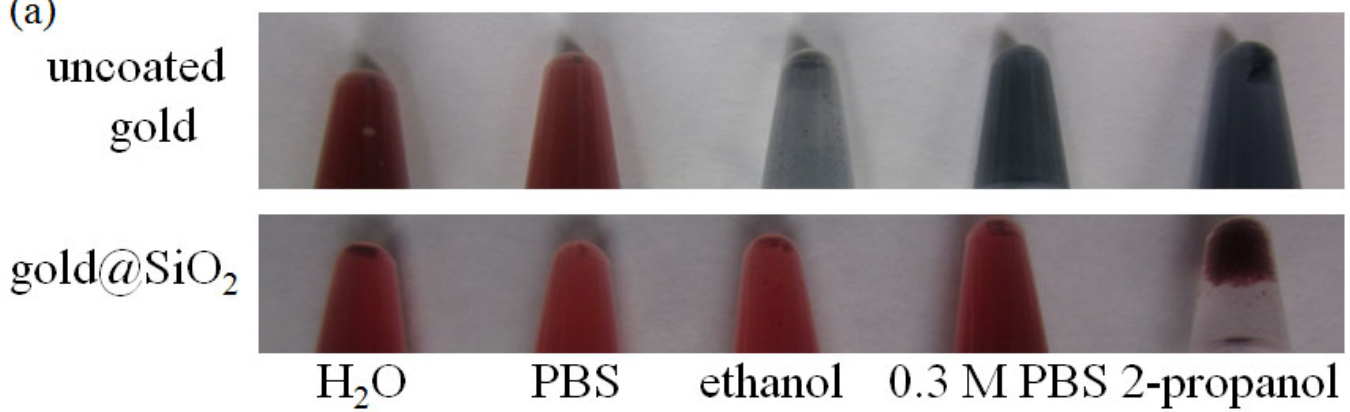

(b)

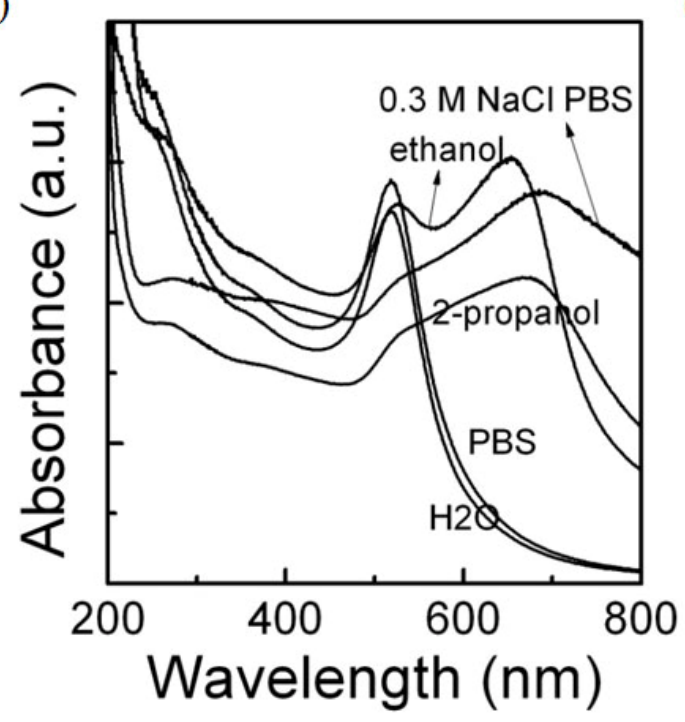

(c)

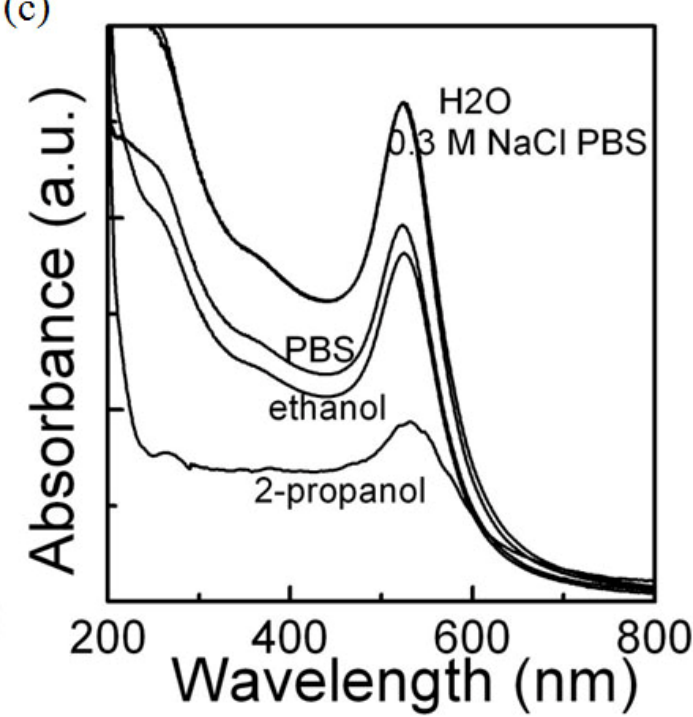

Figure 4.9 (a) Color response and UV-visible aborption spectra of (b) gold nanospheres, and (c) gold nanosphere@MGITC@ $\mathrm{SiO}_{2}$ in representive solvents and salt concentrations.

\subsection{SERS detection of DNA hybridization}

The above results show that gold nanostars are a kind of excellent SERS substrate, and gold nanostar@MGITC@ $\mathrm{SiO}_{2}$ exhibits excellent stability in robust environments. gold nanostar@MGITC@SiO $\mathrm{SiO}_{2}$ should be attractive in a wide range of applications. Herein,we demonstrated that the DNA hybridization can be monitored using SERS based on the present functional particles (Figure 4.10). Firstly, the $\mathrm{SiO}_{2}$ slides were cleaned by successive sonication 
in $\mathrm{CH}_{2} \mathrm{Cl}_{2}$, methanol and D.I. water, followed by heating at $90{ }^{\circ} \mathrm{C}$ in a solution containing $\mathrm{NH}_{4} \mathrm{OH}$ and $\mathrm{H}_{2} \mathrm{O}_{2}$. The cleaned $\mathrm{SiO}_{2}$ slides were immersed into a $200 \mathrm{mM}$ TEPSA ethanolic solution so that the carboxylic group-terminated $\mathrm{SiO}_{2}$ slides were obtained. The carboxylic group terminated $\mathrm{SiO}_{2}$ slides were immersed into a solution with NHS and EDC to activate the carboxylic group, and then amine-modified single stranded DNA was added. As a result, the single stranded DNA is functionalized onto $\mathrm{SiO}_{2}$ slides. Similarly, the single stranded DNA is linked onto gold nanostar@MGITC@SiO was added onto DNA-SiO${ }_{2}$ slides, and incubated for 30 min. After this, the $\mathrm{SiO}_{2}$ slides were rinsed using PBS solution and D.I. water, and used for SERS measurement. For control experiment, the uncomplementary DNA-gold nanostar@MGITC@ $\mathrm{SiO}_{2}$ was used.

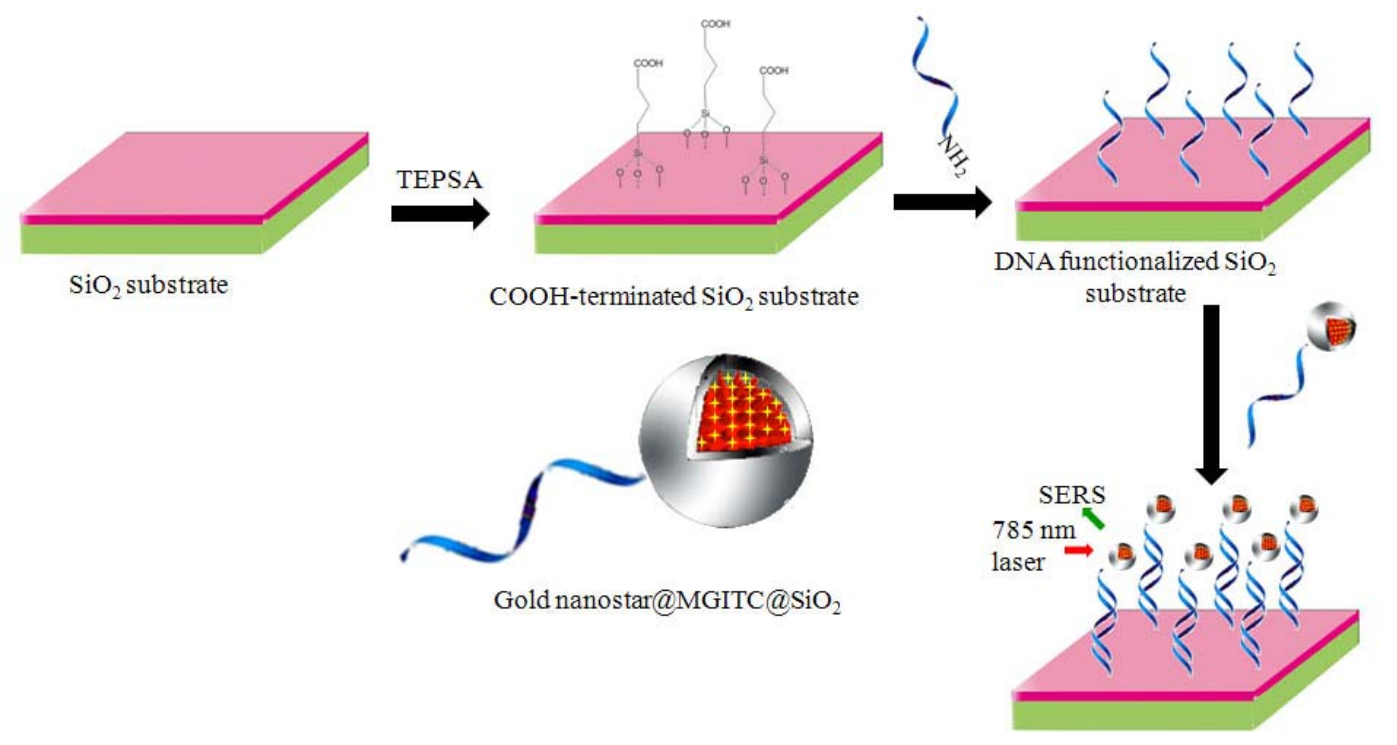

Figure 4.10 Schematical assembly of a SERS device for monitoring of DNA hybridization based on gold nanostar@MGITC@SiO 2. 
Figure 4.11 shows SERS spectra before and after hybridization of DNA on $\mathrm{SiO}_{2}$ slides with complementary and uncomplementary DNA attached on gold nanostar@MGITC@SiO 2 . The DNA-modified $\mathrm{SiO}_{2}$ slides do not show any peak except that the peak around $950 \mathrm{~cm}^{-1}$ is from the $\mathrm{SiO}_{2}$ slide. After hybridization with the complementary DNA attached onto gold nanostar@MGITC@SiO 2 , the strong SERS signal can be seen, which is in agreement with the previous SERS spectra. The control experiment in which the uncomplementary DNA was attached gold nanostar@MGITC@ $\mathrm{SiO}_{2}$ shows no observable SERS peak. Therefore, the present results demonstrate that gold nanostar@MGITC@ $\mathrm{SiO}_{2}$ can be used for monitoring of DNA hybridization. In addition, it is believed that the present detection method can be extended to sensing of other biospecies, such as proteins and viruses.

\subsection{Summary}

In this chapter, we firstly synthesized varous shaped-gold nanoparticles including nanospheres, nanorods and nanostars. Then, we investigated SERS activity of gold nanospheres, nanorods and nanostars under two representative incident lasers of $532 \mathrm{~nm}$ and $785 \mathrm{~nm}$. It was found that the gold nanostars possess the highest SERS activity, and that of the gold nanosphere is the lowest under both $532 \mathrm{~nm}$ and $785 \mathrm{~nm}$ lasers. The three-dimension FDTD simulation shows the significant different electric field distributions among nanospheres, nanorods and nanostars. Under $532 \mathrm{~nm}$ incident laser, the maximum electric field intensity in the gold nanostars is the highest, while under $785 \mathrm{~nm}$ incident laser, the maximum electric field intensity in the gold nanostar still is the highest and that of the gold nanospere is the lowest. The electric field is mainly confined around two ends of nanorods and around these tips of nanostars. Present results indicate that the shape, especially these sharp component in these nanostructures, confines 
and concentrates the LSPR, and consequently generates the SERS activity difference. Gold nanosphere, nanorod and nanostar@MGITC@SiO $\mathrm{Si}_{2}$ were prepared, and their SERS activity and stability were investigated. $\mathrm{SiO}_{2}$ encapsulation of MGITC-tagged gold nanostructures not only prevents MGITC molecules from leaking out to the surrounding environments but also improves the colloidal stability and LSPR stability in the real environment, for example some organic solvents and high salt concentration. Finally, hybridization monitoring of DNA based on gold nanostar@MGITC@SiO $\mathrm{Si}_{2}$ was demonstrated. Therefore, the present result suggests promising applications of gold@MGITC@ $\mathrm{SiO}_{2}$ composite materials in biosensing and environmental monitoring.

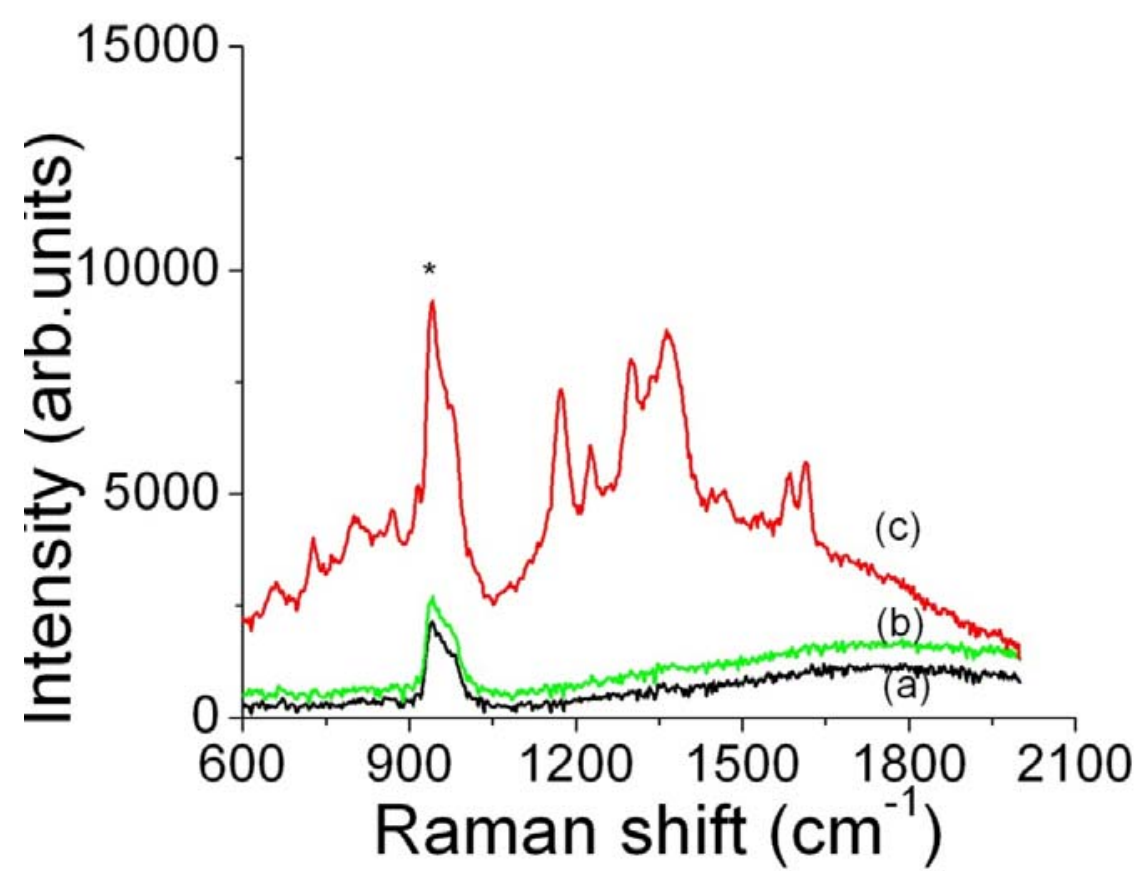

Figure 4.11 SERS spectra of (a) DNA-SiO ${ }_{2}$ slides, (b) uncomplementary gold nanostar@MGITC@SiO $\mathrm{SiO}_{2}$ with $\quad \mathrm{DNA}^{-\mathrm{SiO}_{2}}$ slides, and (c) complementary gold nanostar@MGITC@SiO $\mathrm{Si}_{2}$ with DNA-SiO 2 slides. 


\section{References}

[1] He, D.; Hu, B.; Yao, Q.-F.; Wang, K.; Yu, S.-H. ACS Nano, 2009, 3, 3993-4002.

[2] Xie, L.; Ling, X.; Fang, Y.; Zhang, J.; Liu, Z. J. Am. Chem. Soc., 2009, 131, 9890-9891.

[3] Le Ru, E.C.; Etchegoin, P. G. Principles of Surface-Enhanced Raman Spectroscopy and related plasmonic effects, 1st ed.; Elsevier B. V.: Oxford, 2009.

[4] Ikeda, K.; Suzuki, S.; Uosaki, K. Nano Lett., 2011, 11, 1716-1722.

[5] Zhu, Z.; Meng, H.; Liu, W.; Liu, X.; Gong, J.; Qiu, X.; Jiang, L.; Wang, D.; Tang, Z. Angew. Chem. Int. Ed., 2011, 50, 1593-1596.

[6] Yokota, Y.; Ueno, K.; Misawa, H. small, 2011, 7, 252-258.

[7] Park, W.-H.; Kim, Z. H. Nano Lett., 2010, 10, 4040-4048.

[8] Shanmukh, S.; Jones, L.; Driskell, J.; Zhao,Y.; Dluhy, R.; Tripp, R. A. Nano Lett., 2006, 6, 2630-2636.

[9] Qian, X.; Peng, X. H.; Ansari, D. O.; Yin-Goen, Q.; Chen, G. Z.; Shin, D. M.; Yang, L.; Young, A. N.; Wang, M. D.; Nie, S. Nat. Biotech., 2008, 26, 83-90.

[10]) Lee, S. J.; Moskovits, M. Nano Lett., 2011, 11, 145-150.

[11] Graham, D. Angew. Chem. Int. Ed., 2010, 49, 9325 - 9327.

[12] Barhoumi, A.; Halas, N. J. J. Am. Chem. Soc., 2010, 132, 12792-12793.

[13] Yu, X.; Cai, H.; Zhang, W.; Li, X.; Pan, N.; Luo, Y.; Wang, X.; Hou, J. G. ACS Nano, 2011, $5,952-958$.

[14] Kambhampati, P.; Child, C. M.; Foster, M. C.; Campion, A. J. Chem. Phys., 1998, 108, $5013-5026$.

[15] Xu, H.; Aizpurua, J.; Käll, M.; Apell, P. Phys. Rev. E, 2000, 62, 4318-4324. 
[16] Grand, J.; Lamy de la Chapelle, M.; Bijeon, J.-L.; Adam, P.-M.; Vial, A.; Royer, P. Phys. Rev. B, 2005, 72, 033407.

[17] Lee, K.-S.; El-Sayed, M. A. J. Phys. Chem. B, 2005, 109, 20331-20338.

[18] Guo, H.; Ruan, F.; Lu, L.; Hu, J.; Pan, J.; Yang, Z.; Ren, B. J. Phys. Chem. C, 2009, 113, $10459-10464$.

[19] Zuloaga, J.; Prodan, E.; Nordlander, P. ACS Nano, 2010, 4, 5269-5276.

[20] Sabur, A.; Havel, M.; Gogotsi, Y. J. Raman Spectrosc., 2008, 39, 61-67.

[21] Kelly, K. L.; Coronado, E.; Zhao, L. L.; Schatz, G. C. J. Phys. Chem. B, 2003, 107, 668677.

[22] Sant'Ana, A. C.; Rocha, T. C. R.; Santos, P. S.; Zanchetb, D.; Temperini, M. L. A. J. Raman Spectrosc., 2009, 40, 183-190.

[23] Zhang, P.; Guo, Y. J. Am. Chem. Soc., 2009, 131, 3808-3809.

[24] Dondapati, S. K.; Sau, T. K.; Hrelescu, C.; Klar, T. A.; Stefani, F. D.; Feldmann, J. ACS Nano, 2010, 4, 6318-6322.

[25] Khoury, C. G.; Vo-Dinh, T. J. Phys. Chem. C, 2008, 112, 18849-18859.

[26] Banaee, M. G.; Crozier, K. B. ACS Nano, 2011, 5, 307-314.

[27] Qian, X.; Zhou, X.; Nie, S. J. Am. Chem. Soc., 2008, 130, 14934-14935.

[28] Lim, D.-K.; Jeon, K.-S.; Kim, H. M.; Nam, J.-M.; Suh, Y. D. Nat. Mater., 2010, 9, 60-67.

[29] Küstner, B.; Gellner, M.; Schütz, M.; Schöppler, F.; Marx, A.; Ströbel, P.; Adam, P.; Schmuck, C.; Schlöcker, S. Angew. Chem. Int. Ed., 2009, 48, $1950-1953$.

[30] Sanles-Sobrido, M.; Exner, W.; Rodríguez-Lorenzo, L.; Rodríguez-González, B.; CorreaDuarte, M. A.; Álvarez-Puebla, R. A.; Liz-Marzán, L. M. J. Am. Chem. Soc., 2009, 131, 26992705. 
[31] Basu, S.; Pande, S.; Jana, S.; Bolisetty, S.; Pal, T. Langmuir, 2008, 24, 5562-5568.

[32] Huang, C. C.; Chiang, C. K.; Lin, Z. H.; Lee, K. H.; Chang, H. T. Anal. Chem., 2008, 80, 1497-1504.

[33] Gole, A.; Murphy, C. J. Chem. Mater., 2004, 16, 3633-3640.

[34] Barbosa, S.; Agrawal, A.; Rodríguez-Lorenzo, L.; Pastoriza-Santos, I.; Alvarez-Puebla, R. A.; Kornowski, A.; Weller, H.; Liz-Marzán, L. M. Langmuir, 2010, 26, 14943-14950.

[35] Brown, M. B.; Suteewong, T.; Kumar, R. S. S.; D’Innocenzo, V.; Petrozza, A.; Lee, M.; Wiesner, U.; Snaith, H. J. Nano Lett., 2011, 11, 438-445.

[36] Obare, S. O.; Jana, N. R.; Murphy, C. J. Nano Lett., 2001, 1, 601-603.

[37] Sendroiu, I. E.; Warner, M. E.; Corn, R. M. Langmuir, 2009, 25, 11282-11284.

[38] Oskooi, A. F.; Roundy, D.; Ibanescu, M.; Bermel, P.; Joannopoulos, J. D.; Johnson, S. G. MEEP: A flexible free-software package for electromagnetic simulations by the FDTD method. Comput. Phys. Commun., 2010, 181, 687-702.

[39] Johnson, P. B.; Christy, R. W. Optical Constants of the Noble Metals. Phys. Rev. B, 1972, 6, $4370-4379$.

[40] Franzen, S.; Folmer, J. C. W.; Glomm, W. R.; O'Neal, R. J. Phys. Chem. A, 2002, 106, $6533-6540$.

[41] Doering, W. E.; Nie, S. Anal. Chem. 2003, 75, 6171-6176.

[42] Kamińska, A.; Dzięcielewski, I.; Weyher, J. L.; Waluk, J.; Gawinkowski, S.; Sashuk, V.; Fiałkowski, M.; Sawicka, M.; Suski, T.; Porowski, S.; Hołyst, R. J. Mater. Chem., 2011, 21, $8662-8669$.

[43] Lueck, H. B.; Daniel, D. C.; McHale, J. L. J. Raman Spectrosc., 1993, 24, 363-370.

[44] Domke, K. F.; Zhang, D.; Pettinger, B. J. Am. Chem. Soc., 2006, 128, 14721-14727. 
[45] Le Ru, E. C.; Blackie, E.; Meyer, M.; Etchegoin, P. G. J. Phys. Chem. C, 2007, 111, 1379413803.

[46] Henzie, J.; Barton, J. E.; Stender, C. L.; Odom, T. W. Acc. Chem. Res., 2006, 39, 249-257.

[47] Wang, W.; Li, Z.; Gu, B.; Zhang, Z.; Xu, H. ACS Nano, 2009, 3, 3493-3496.

[48] Ruan, C.; Wang, W.; Gu, B. J. Raman Spectrosc., 2007, 38, 568-573.

[49] Blackie, E. J.; Le Ru, E. C.; Etchegoin, P. G. J. Am. Chem. Soc., 2009, 131, 14466-14472.

[50] Yang, L.; Jiang, X.; Ruan, W.; Zhao, B.; Xu, W.; Lombardi, J. R. J. Phys. Chem. C, 2008, 112, 20095-20098.

[51] Gutés, A.; Carraro, C.; Maboudian, R. J. Am. Chem. Soc., 2010, 132, 1476-1477.

[52] Kim, N. H.; Lee, S. J.; Moskovits, M. Nano Lett., 2010, 10, 4181-4185.

[53] Chen, G.; Wang, Y.; Yang, M.; Xu, J.; Goh, S. J.; Pan, M.; Chen, H. J. Am. Chem. Soc., 2010, 132, 3644-3645.

[54] Li, W.; Camargo, P. H. C.; Lu, X.; Xia, Y. Nano Lett., 2009, 9, 485-490. 


\section{CHAPTER 5: SERS DETECTION OF ATP BASED ON GOLD NANOSTAR@RAMAN-REPORTER@SILICA SANDWICHED NANOPARTICLES}

\subsection{Introduction}

The development of rapid, facile and sensitive methods for detection of small organic molecules such as cocaine, 2,4,6-trinitrotoluene (TNT), dopamine and adenosine, is extremely desirable in environmental, analytical, and forensic sciences as well as therapeutics and diagnostics. Currently, a wide range of strategies such as Förster resonance energy transfer (FRET) [1,2], colorimetric response [3-6], electrochemical methods [7-10] and optical surface plasmon resonance[11] have been demonstrated feasible for quantitative detection of small molecules. However, most of these techniques suffer drawbacks of low sensitivity, timeconsuming, poor reproducibility or laboratory-based operation, etc. although great achievements have been obtained. In recent decades, surface-enhanced Raman spectroscopy is emerging as a powerful detection tool for unique advantages [12-18], including (i) providing spectral fingerprinting information of molecules, (ii) potential multiplexing detection capability under a single laser excitation due to the result of the narrow linewidth of vibrational Raman bands, (iii) ultrahigh sensitivity, allowing single-molecule detection, (iv) easy measurement operation without complicated sample preparation, rendering the possibility of on-site detection, and (v) nondestructive detection that allows for identification of analytes in a wide variety of matrices. Therefore, very high selectivity and sensitivity endowed by surface-enhanced Raman scattering 
(SERS), along with the highly informative spectrum characteristics of Raman spectroscopy, allows SERS-based method a feasible alternative to conventional methods mentioned above for detection of small molecules.

SERS has been extensively investigated as an efficient sensing strategy for diagnosis, bioimaging, chemical detection and environmental monitoring. High sensitive detection of virus, DNA, TNT, adenosine and heavy metallic ions (e.g. mercuric (II)) has been realized based on SERS methods [19-23]. In typical SERS assays, Raman reporters attached to metallic substrates are used to provide the Raman signal, which indirectly responds to the analytes. Most of the previous work directly adsorbed Raman reporters onto the surface of plasmonic SERS substrates [20]. This kind of SERS assay suffers poor reproducibility of Raman signals due to desorption of Raman reporters from the surface of substrates, upon direct exposure to the robust surrounding environments. In addition, the commonly used SERS substrates, typically gold or silver, are covered by the surface ligands, and thus are unfavorable for the desired surface functionalization, which is not suitable for biological applications. In this context, the search for more effective and flexible strategies for detection of various analytes is of great practical significance and in high demand. In Chapter 4, we have shown that gold nanostars exhibited extremely high electromagnetic enhancement for SERS of attached molecules and are excellent SERS substrates for chemical detection. Also, it was demonstrated that $\mathrm{SiO}_{2}$ encapsulated gold nanostar@malachite green isothiocyanate (MGITC) (gold nanostar@MGITC@ $\mathrm{SiO}_{2}$ ) sandwiched structure renders superior stability and reproducibility of Raman signals because the protective $\mathrm{SiO}_{2}$ layer prevents Raman reporter, MGITC, from leaking out. More importantly, encapsulation of a large number of MGITC moclues inside $\mathrm{SiO}_{2}$ layer renders huge signaling 
amplification ability. Therefore, use of gold nanostar@MGITC@ $\mathrm{SiO}_{2}$ is expected to benefit to improvement of the performance of SERS sensors for detection of small molecules.

Adenosine triphosphate (ATP), a universal energy carrier in biological systems, plays a crucial role in the regulation of cellular metabolism and biochemical pathways in cell physiology [24]. Therefore, the quantitative detection of ATP is essential in biochemistry and clinic diagnosis. As an assay paradigm using gold nanostar@MGITC@ $\mathrm{SiO}_{2}$ as signaling probes, quantitative detection of ATP was investigated by a SERS approach. In this assay, the ATPbinding aptamer, which possesses high affinity and selectivity for ATP, was used as the capture probe [25]. After hybridization of complementary DNA immobilized onto a gold film with the aptamer capture probe labeled on gold nanostar@MGITC@ $\mathrm{SiO}_{2}$, SERS sensor exhibits strong Raman signal of MGITC. The gold nanostar@MGITC@ $\mathrm{SiO}_{2}$-labeled capture DNA was dissociated upon addition of ATP analytes, and consequently the SERS sensor shows an ultrasensitive response to the ATP concentration with a $12.4 \mathrm{pM}$ detection limit.

\subsection{Materials and experimental methods}

\subsubsection{Chemicals and Reagents}

MGITC was purchased from Molecular Probes. 3-triethoxylsilylpropyl succinic anhydride (TEPSA) was purchased from Gelest Inc. DNA sequences of 3'- $\mathrm{NH}_{2}-\left(\mathrm{CH}_{2}\right)_{3}-\mathrm{TGG}$ AAG GAG GCG TTA TGA GGG GGT CCA CG-5' and 5'- $\mathrm{NH}_{2}-\left(\mathrm{CH}_{2}\right)_{6}-\mathrm{GCA}$ CCT TCC TCC GCA ATA CTC CCC CAG GTG C-3' were obtained from Eurofins MWG Operon (Huntsville, AL). $\mathrm{Na}_{2} \mathrm{HPO}_{4}(99.0 \%)$ and $\mathrm{NaH}_{2} \mathrm{PO}_{4}(99.0 \%)$ came from Alfa Aesar. $\mathrm{N}$-hydroxysuccinimide (NHS), 1-ethyl-3-(3-dimethylaminopropyl)-carbodiimide (EDC), 11-mercaptoundecanoic acid (MUA), 
11-mercapto-1-undecanol ethanolic (MU), adenosine triphosphate (ATP), cytidine triphosphate (CTP), and guanosine triphosphate (GTP) were purchased from Sigma-Aldrich. All solvents were of analytical grade and used without further purification. Deionized (D.I.) water was purified using a Milli-Q Millipore system (18.2 M $/ / \mathrm{cm}^{2}$, Millipore Corp., USA).

\subsubsection{Apparatus}

Scanning electron microscope (SEM) measurements were performed using a field emission JSM-7600F microscope, respectively. Raman measurements were conducted in a Renishaw InVia Raman spectrometer at an excitation laser of $532 \mathrm{~nm}$. A microscope equipped with $20 \mathrm{x}$ objective was used to focus the incident excitation laser. The laser power on the sample was 50 $\mathrm{mW}$, and the accumulation time is $10 \mathrm{~s}$.

\subsubsection{DNA functionalization of gold nanostar@MGITC@SiO2}

Gold nanostar@MGITC@ $\mathrm{SiO}_{2}$ was synthesized following the previous described method in Chapter 4.2, except the increasing addition of MGITC $\left(2.5 \times 10^{-7} \mathrm{M}\right)$ in order to enhance the Raman signal. $200 \mu \mathrm{L}$ TEPSA (200 mM) was added to $400 \mu \mathrm{L}$ gold nanostar@MGITC@ $\mathrm{SiO}_{2}$, and incubated overnight. COOH-terminated gold nanostar@MGITC@ $\mathrm{SiO}_{2}$ was achieved after centrifugation and re-dispersed in $200 \mu \mathrm{L}$ PBS solution for further use. The functionalization of DNA was carried out by the carbodiimide chemistry [26]. First, $100 \mu \mathrm{L}$ solution containing 50 mM NHS and 200 mM EDC was added to $200 \mu \mathrm{L}$ gold nanostar@MGITC@SiO ${ }_{2}$ PBS solution in order to activate the $\mathrm{COOH}$ group, and then $50 \mu \mathrm{L} 0.1 \mathrm{mM}$ DNA sequence of 3'- $-\mathrm{NH}_{2}-\left(\mathrm{CH}_{2}\right)_{3}{ }^{-}$ TGG AAG GAG GCG TTA TGA GGG GGT CCA CG-5' (capture DNA) was added after incubation for $2 \mathrm{~h}$. After incubated overnight, the solution was centrifuged and washed using a 
PBS solution to obtain the DNA-functionalized particles, which was dispersed in $800 \mu \mathrm{L}$ PBS solution for further use.

\subsubsection{Preparation of capture DNA-modified substrates}

Striped-gold films were prepared by metal vapor deposition on $\mathrm{Si}$ substrates with a thin $\mathrm{Cr}$ layer as the adhesion layer. The resulting gold film was cleaned with successive immersion in $\mathrm{CH}_{2} \mathrm{Cl}_{2}$, ethanol and D.I. water for $10 \mathrm{~min}$, respectively, and heated at $90{ }^{\circ} \mathrm{C}$ in a $20 \mathrm{~mL}$ peroxide solution for $1 \mathrm{~h}$. Following this, gold films were successively washed with ethanol and D.I. water, respectively, and dried at $60{ }^{\circ} \mathrm{C}$ in a vacuum oven. The cleaned gold films were incubated overnight in a solution containing $100 \mathrm{mM}$ MUA and $100 \mathrm{mM} \mathrm{MU}$, and then washed using ethanol and D.I. water, respectively. The resulting MUA/MU-modified gold film was activated by immersion in a solution containing $50 \mathrm{mM}$ NHS and $200 \mathrm{mM}$ EDC in a PBS solution (10 $\mathrm{mM}, \mathrm{pH}$ 7.0). The activated MUA/MU-modified gold film was incubated overnight in a PBS solution containing $0.1 \mathrm{mM}$ DNA with sequence of $5^{\prime}-\mathrm{NH}_{2}-\left(\mathrm{CH}_{2}\right)_{6}$-GCA CCT TCC TCC GCA ATA CTC CCC CAG GTG C-3' (complementary DNA). After immobilization of the complementary DNA, the gold film was successively rinsed with D.I. water and PBS solution to remove excess DNA.

\subsubsection{Assembly of the SERS Sensor and its application for ATP Detection}

The complementary DNA-modified gold substrates were immersed in the DNAfunctionalized gold nanostar@MGITC@ $@ \mathrm{SiO}_{2}$ solution obtained above. After incubated at about $40{ }^{\circ} \mathrm{C}$ in a water bath for $1 \mathrm{~h}$, the gold films were washed with $10 \mathrm{mM}$ PBS. The resulting substrates were modified by double-stranded DNA (dsDNA) labeled with gold nanostar@MGITC@SiO $\mathrm{Si}_{2}$ as the signaling probe. 
In a typical assay, the SERS sensors were immersed in a solution of various ATP (CTP or GTP) concentrations, and incubated for $30 \mathrm{~min}$. The substrates were rinsed by $10 \mathrm{mM}$ PBS, and subjected to the measurement by the SERS spectroscopy using an accumulation time of $10 \mathrm{~s}$.

\subsection{Design principle of SERS sensors}

DNA and RNA aptamers have been well-developed as recognition elements for small molecule detection, which renders excellent selectivity for the specific binding to the analytes $[8,25,27]$. Here, we used the well-known ATP-binding DNA aptamer as the recognition probe into which two ATP molecules can intercalate by forming noncanonical G:A base pairs [25]. The preparation of the SERS sensor is schematically shown in Figure 5.1a. The gold film is firstly modified with the carboxylic group via a thiolate group, and then a hybridized dsDNA aptamer is anchored to the gold film. The gold film surface is passivated with MU to prevent functional particles from binding to the free gold surface sites. The capture DNA for ATP recognition is labeled by the $\mathrm{COOH}$-terminated gold nanostar@MGITC@ $\mathrm{SiO}_{2}$, and hybridization of the complementary DNA on the gold film with the capture DNA results in immobilization of the gold nanostar@MGITC@ $\mathrm{SiO}_{2}$. Finally, this preparation process results in the gold film with a homogeneous coating of gold nanostar@MGITC@ $\mathrm{SiO}_{2}$. The "hot spot" formed between the gold nanoparticle and the gold film enhances the Raman signal of MGITC. Strong Raman spectra from MGITC can be observed due to incorporation of a large number of MGITC molecules and its large Raman cross-section. As addition of the target ATP, ATP will interact with the capture DNA labeled by the nanoparticles, which results in dehybridization of dsDNA and dissociation of capture DNA with the nanoparticle signaling probe (Figure 5.1b). Thus, the dissociation of capture DNA greatly results in the reduced Raman signal. 
(a)

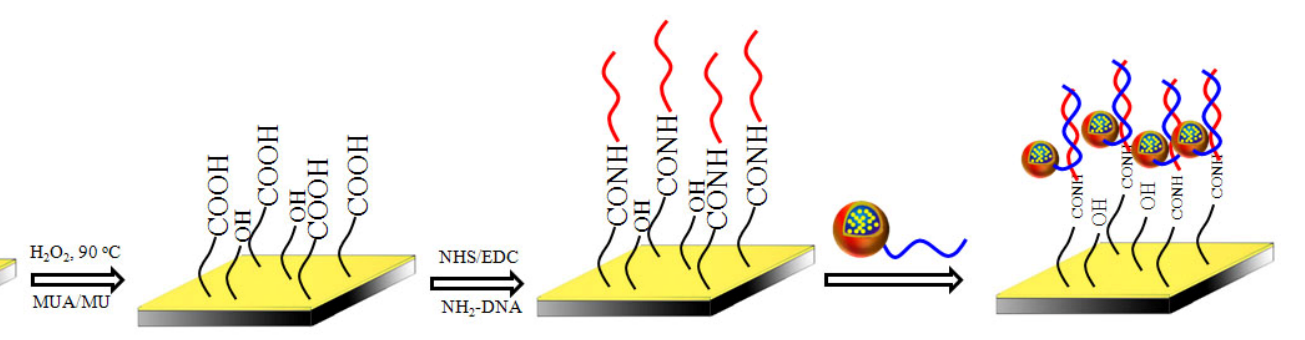

(b)
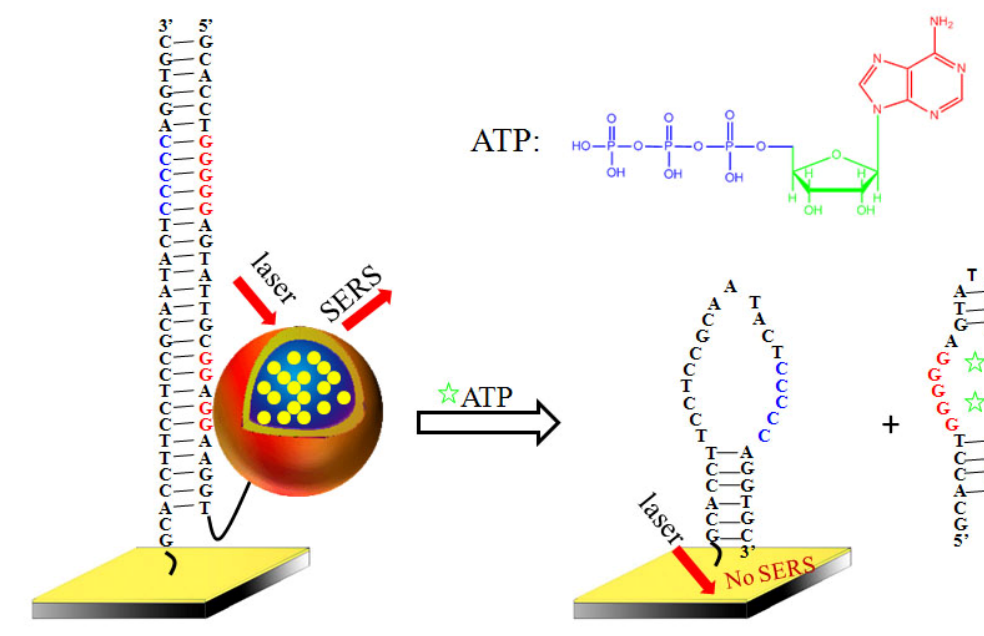

Aptamer: 3'- $\mathrm{NH}_{2}-\left(\mathrm{CH}_{2}\right)_{3}$-TGGAAGGAGGCGTTATGAGGGGGTCCACG-5'

Complementary DNA: 5'- $\mathrm{NH}_{2}-\left(\mathrm{CH}_{2}\right)_{6}$-GCACCTTCCTCCGCAATACTCCCCCAGGTGC-3'

Figure 5.1 Schematic illustrations of (a) preparation procedure of the SERS sensor based on gold nanostar@MGITC@ $\mathrm{SiO}_{2}$ as signaling probe and (b) operation principle of the SERS sensor for ATP detection. Inset in (b) shows the molecular structure of ATP.

\subsection{SERS measurement}

Following this, we checked the Raman spectra on the gold substrates before and after gold nanostar@MGITC@SiO $\mathrm{SiO}_{2}$ deposition (Figure 5.2). No Raman band associated with MGITC molecules was observed on the only complementary DNA-modified gold substrate while after hybridization with capture DNA-gold nanostar@MGITC@SiO $\mathrm{SiO}_{2}$, strong Raman bands were observed from MGITC, in agreement with that in the free standing gold 
nanostar@MGITC@SiO . The assignments of Raman bands are listed in Table 5.1. Peaks at 1618,1589 and $1366 \mathrm{~cm}^{-1}$ are attributed to the phenyl-N stretching mode, in-plane ring stretching and bending modes, and aromatic ring stretching mode, respectively $[28,29]$. The 1296, 1174 and $912 \mathrm{~cm}^{-1}$ peaks are assigned to in-plane C-H and C-C-H bending mode, N-C stretching mode and $B_{1 \mathrm{u}}$ in-plane benzene ring modes, respectively $[28,29]$. Furthermore, the SERS spectra were investigated upon exposure of the assembled sensor to a ATP solution with a concentration of $100 \mathrm{nM}$. There is no observable SERS bands associated with the MGITC molecule, which is attributed to dehybridizaion of dsDNA by ATP and consequently disappears of SERS signals.

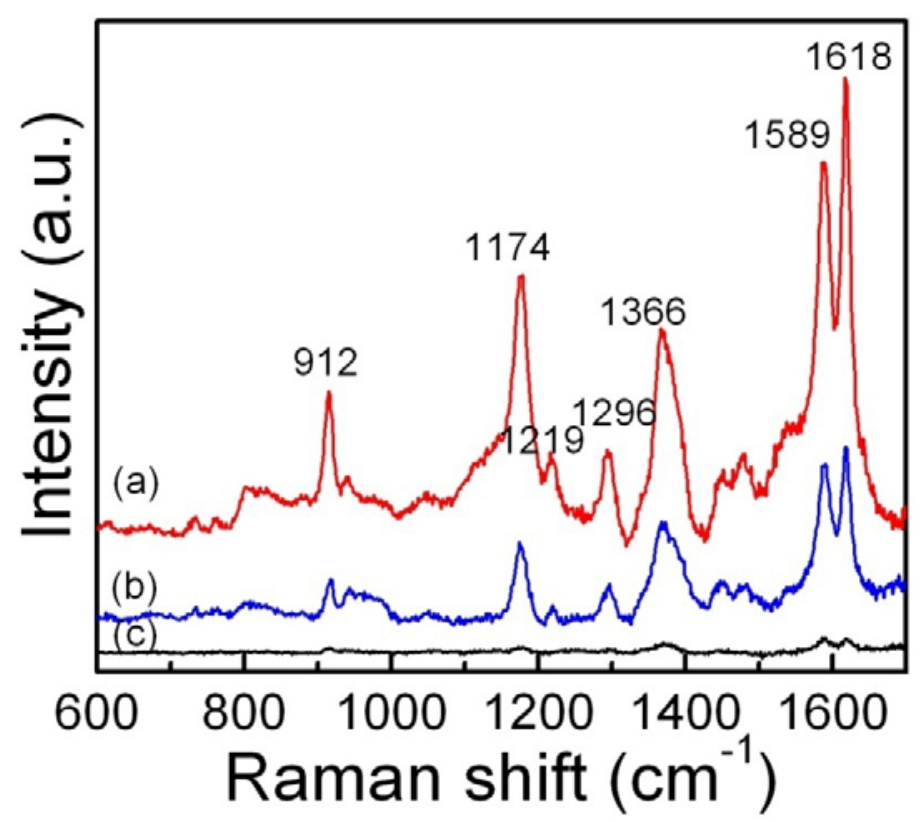

Figure 5.2 Raman spectra of (a) gold nanostar@MGITC@ $\mathrm{SiO}_{2}$ in solution, (b) the SERS sensor with gold nanostar@MGITC@ $\mathrm{SiO}_{2}$ as signaling probe and (c) the SERS sensor after exposure to $100 \mathrm{nM}$ ATP. 
Table 5.1 Assignments of selected Raman bands of MGITC in SERS spectra

\begin{tabular}{cl}
\hline Peak position $\left(\mathrm{cm}^{-1}\right)$ & \multicolumn{1}{c}{ Assignment } \\
1618 & Phenyl-N and C-C stretching \\
1589 & In-plane ring stretching and bending \\
1366 & Aromatic ring stretching \\
1296 & In-plane C-H and C-C-H bending \\
1219 & N-C stretching mode \\
1174 & In-plane C-H bending \\
912 & $\mathrm{~B}_{1 \mathrm{u}}$ in plane benzene ring \\
\hline
\end{tabular}

\subsection{Sensitivity of SERS sensor toward ATP}

On the above analysis and the fact that each ATP aptamer molecule can specifically bind to two ATP molecules $[25,30]$, we conclude that the present system should have a concentrationdependent response of SERS and can be used for ATP detection. The sensitivity of the present SERS sensor was examined in a series of solutions with various ATP concentrations $(0-1 \mu \mathrm{M})$. Figure 5.3a shows SERS spectra in the presence of various ATP concentrations. At low concentrations, the SERS spectra are characteristic of the Raman spectrum of MGITC, similar to that in Figure 5.2. All peaks decrease in intensity with the increasing ATP concentration, and there is almost no observable Raman signals associated with MGITC when the ATP concentration is $10 \mathrm{nM}$, which suggests that most of particles are removed. The ratio of Raman peak intensities at $1618 \mathrm{~cm}^{-1}$ in the presence and absence of ATP $\left(I / I_{0}\right)$ was used for the quantitative evaluation of ATP concentration (Figure 5.3b-c). In the absence of ATP, the peak intensity ratio is the highest, decreases with the increase in the ATP concentration, and finally reaches to the saturation value at the concentration higher than $1 \mathrm{nM}$. The detection limit, which 
is defined as the analyte concentration that produces a signal three times larger than the standard deviation of blank measurements, is determined to be $12.4 \mathrm{pM}$. This sensitivity is significantly higher than previous electrochemical $[8,10]$, fluorescent and colorimetric sensors [31-33] based on DNA aptamers as the recognition element. Furthermore, a linear range of $1618 \mathrm{~cm}^{-1}$ peak intensity ratio vs $\log [\mathrm{ATP}]$ is shown at the low concentration region (Figure 5.3c). The high sensitivity in the SERS sensor is ascribed to several advantages including (i) the strong electromagnetic enhancement of Raman signals by gold nanostars and (ii) signal amplification of a large number of MGITC molecules in the sandwiched structure.

\subsection{Selectivity of SERS sensor toward ATP}

In order to evaluate the selectivity of the present SERS sensor toward ATP, control experiments were conducted by incubating the SERS sensor in GTP or CTP solutions with various concentrations, respectively (Figures 5.3c and 5.4). It can be also seen that exposure to different concentrations of GTP and CTP does not decrease the Raman signal significantly, which is mainly due to the fact that both GTP and CTP cannot interact with DNA aptamer through the G:A base pairs occurring between DNA aptamer and ATP, and thus fail to separate the duplex DNA. Therefore, the present SERS sensor is very specific to ATP because of the high selectivity of DNA aptamer toward ATP. 


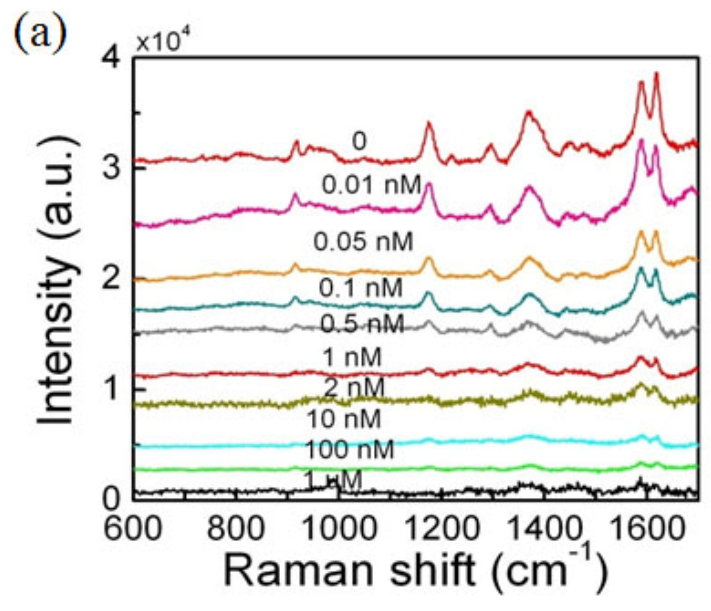

(b)

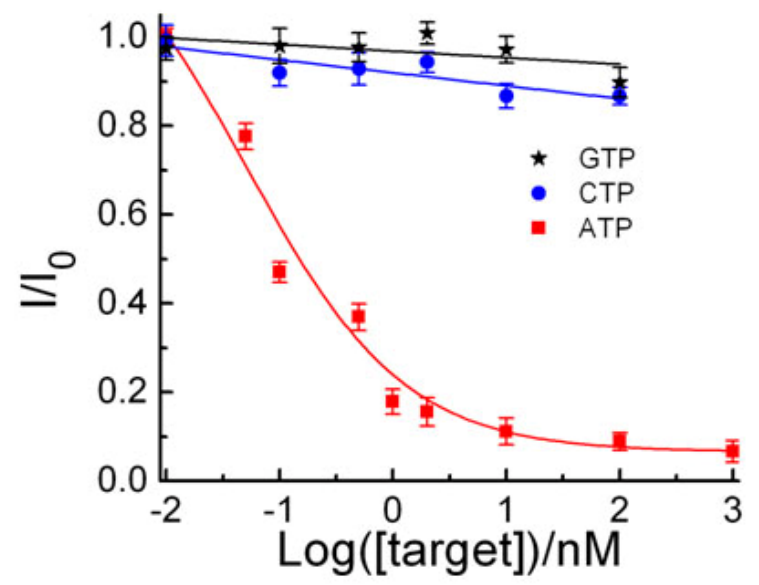

(c)

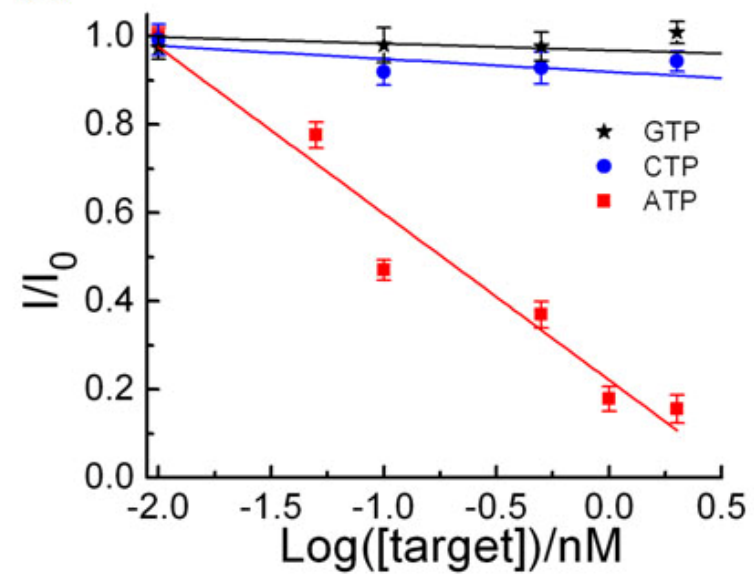

Figure 5.3 (a) SERS spectra of MGITC as a function of ATP concentration in the SERS sensor based on gold nanostar@MGITC@ $\mathrm{SiO}_{2}$ as signaling probe, (b) concentration-dependent 
response of the Raman intensity to the initial Raman intensity ratio at $1618 \mathrm{~cm}^{-1}$, and (c) the linear range in the low concentration region of (b).

In addition, the present system could be regenerated due to its unique design of the complementary DNA sequence, the first six bases of whose 5'- and 3'-teminus are complementary. The signal amplification can be enhanced through increasing amount of Raman signaling molecules, MGITC. More importantly, $\mathrm{SiO}_{2}$ encapsulation of MGITC endows the present assay system excellent stability and reproducibility.
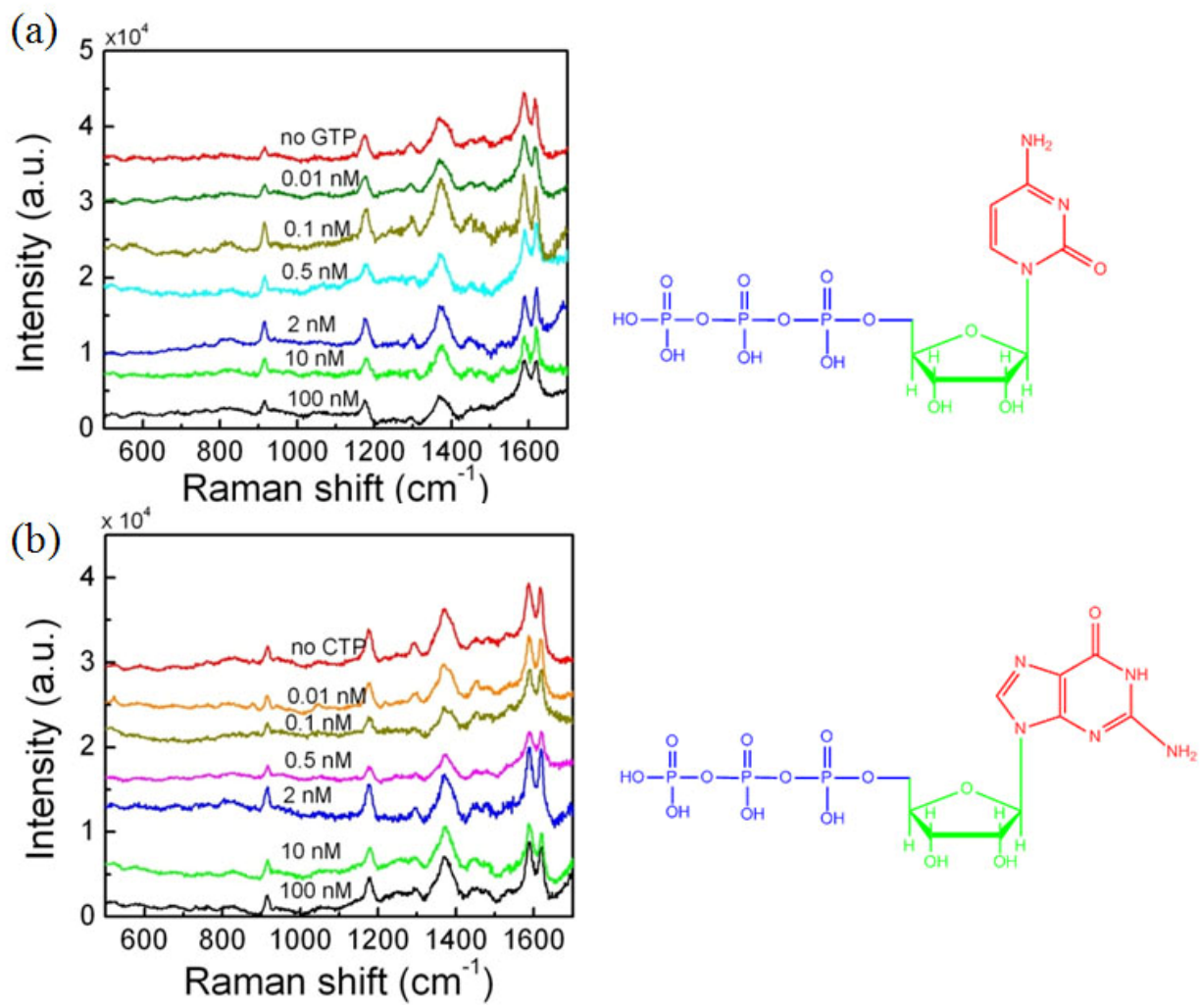

Figure 5.4 SERS spectra of the SERS sensor based on the gold nanostar@MGITC@ $\mathrm{SiO}_{2}$ as signaling probe after exposure to solutions with various concentrations of (a) GTP and (b) CTP. 


\subsection{Summary}

This chapter is dedicated to developing a SERS sensor for ATP detection using gold nanostar@MGITC@ $\mathrm{SiO}_{2}$ as the signaling probe. First, the operation principle of the SERS sensor was introduced. ATP-binding DNA aptamer was used as the recognition element. Second, the sensitivity toward ATP was investigated. The huge signaling amplification ability of gold nanostar@MGITC@SiO $\mathrm{Sin}_{2}$ sandwiched composite materials allows the high sensitivity detection. It has been demonstrated that this assay approach provides a facile detection of ATP at a 12.4 pM detection limit. Then, the selectivity was investigated. The specific binding of aptamer toward ATP renders high selectivity over its analogues such as GTP and CTP. In addition, encapsulation of a large number of MGITC molecules inside $\mathrm{SiO}_{2}$ shell produces strong Raman signaling amplification ability, contributing to the high sensitivity as well. Furthermore, the gold nanostar@MGITC@SiO $\mathrm{Si}_{2}$ sandwiched structure improves assay stability and reproducibility. With selection of specific aptamer or other recognition elements, this assay approach can be expectedly extended to detection of a wide range of analytes.

\section{References}

[1] Goldman, E. R.; Medintz, I. L.; Whitley, J. L.; Hayhurst, A.; Clapp, A. R.; Uyeda, H. T.;

Deschamps, J. R.; Lassman, M. E.; Mattoussi. H. J. Am. Chem. Soc., 2005, 127, 6744-6751.

[2] Endoh, T.; Shintani, R.; Mie, M.; Kobatake, E.; Ohtsuki, T.; Sisido, M. Bioconjugate Chem., 2009, 20, 2242-2246.

[3] Forzani, E. S.; Lu, D.; Leright, M. J.; Aguilar, A. D.; Tsow, F.; Iglesias, R. A.; Zhang, Q.; Lu, J.; Li, J.; Tao, N. J. J. Am. Chem. Soc., 2009, 131, 1390-1391. 
[4] Xu, X.; Wang, J.; Yang, F.; Jiao, K.; Yang, X. small, 2009, 5, 2669-2672.

[5] Kong, B.; Zhu, A.; Luo, Y.; Tian, Y.; Yu, Y.; Shi, G. Angew. Chem. Int. Ed., 2011, 50, 1837 $-1840$.

[6] Jiang, Y.; Zhao, H.; Lin, Y.; Zhu, N.; Ma, Y.; Mao, L. Angew. Chem. Int. Ed., 2010, 49, 4800 $-4804$.

[7] Aguilar, A. D.; Forzani, E. S.; Leright, M.; Tsow, F.; Cagan, A.; Iglesias, R. A.; Nagahara, L. A.; Amlani, I.; Tsui, R.; Tao, N. J. Nano Lett., 2010, 10, 380-384.

[8] Zayats, M.; Huang, Y.; Gill, R.; Ma, C.; Willner, I. J. Am. Chem. Soc., 2006, 128, 1366613667.

[9] Baker, B. R.; Lai, R. Y.; Wood, M. S.; Doctor, E. H.; Heeger, A. J.; Plaxco, K. W. J. Am. Chem. Soc., 2006, 128, 3138-3139.

[10] Zuo, X.; Xiao, Y.; Plaxco, K. W. J. Am. Chem. Soc., 2009, 131, 6944-6945.

[11] Wang J.; Zhou, H. S. Anal. Chem., 2008, 80, 7174-7178.

[12] Cao, Y. W. C.; Jin, R.; Mirkin, C. A. Science, 2002, 297, 1540.

[13] Kneipp, K.; Wang, Y.; Kneipp, H.; Perelman, L. T.; Itzkan, I.; Dasari, R.R.; Feld, M. S. Phys. Rev. Lett., 1997, 78, 1667-1670.

[14] Nie, S.; Emory, S. R. Science, 1997, 275, 1102-1106.

[15] Kim, N. H.; Lee, S. J.; Moskovits, M. Nano Lett., 2010, 10, 4181-4185.

[16] Tognalli, N. G.; Cortés, E.; Hernández-Nieves, A. D.; Carro, P.; Usaj, G.; Balseiro, C. A.; Vela, M. E.; Salvarezza, R. C.; Fainstein, A. ACS Nano, 2011, 5, 5433-5443.

[17] Ochsenkühn, M. A.; Jess, P. R. T.; Stoquert, H.; Dholakia, K.; Campbell, C. J. ACS Nano, 2009, 3, 3613-3621. 
[18] Chen, G.; Wang, Y.; Yang, M.; Xu, J.; Goh, S. J.; Pan, M.; Chen, H. J. Am. Chem. Soc., 2010, 132, 3644-3645.

[19] Barhoumi, A.; Halas, N. J. J. Am. Chem. Soc., 2010, 132, 12792-12793.

[20] Dasary, S. S. R.; Singh, A. K.; Senapati, D.; Yu, H.; Ray, P. C. J. Am. Chem. Soc., 2009, $131,13806-13812$.

[21] Han, D.; Sung Lim, Y.; Kim, B. J.; Piao L.; Chung, T. D. Chem. Commun., 2010, 46, 55875589.

[22] Wang, G.; Lipert, R. J. Anal. Chem., 2011, 83, 2554-2561.

[23] Shanmukh, S.; Jones, L.; Driskell, J.; Zhao,Y.; Dluhy, R.; Tripp, R. A. Nano Lett., 2006, 6, 2630-2636.

[24] Ross, J. J. Phys. Chem. B, 2006, 110, 6987-6990.

[25] Liu, J.; Cao, Z.; Lu, Y. Chem. Rev., 2009, 109, 1948-1998.

[26] Wang , H.; Xu , W.; Zhang , H.; Li , D.; Yang, Z.; Xie , X.; Li ,T.; Liu, X. small, 2011, 7 , $1987-1992$.

[27] Yao, W.; Wang, L.; Wang, H.; Zhang, X.; Li, L. Biosensors and Bioelectronics, 2009, 24, $3269-3274$.

[28] Kamińska, A.; Dzięcielewski, I.; Weyher, J. L.; Waluk, J.; Gawinkowski, S.; Sashuk, V.; Fiałkowski, M.; Sawicka, M.; Suski, T.; Porowski, S.; Hołyst, R. J. Mater. Chem., 2011, 21, $8662-8669$.

[29] Lueck, H. B.; Daniel, D. C.; McHale, J. L. J. Raman Spectrosc., 1993, 24, 363-370.

[30] Huizenga, D. E.; Szostak, J. W. Biochemistry, 1995, 34, 656-665.

[31] Wang, Y.; Wang, Y.; Liu, B. Nanotechnology, 2008, 19, 415605.

[32] Wang, J.; Jiang, Y.; Zhou, C.; Fang, X. Anal. Chem., 2005, 77, 3542-3546. 
[33] Liu, F.; Zhang, J.; Chen, R.; Chen, L.; Deng, L. Chem. Biodiv., 2011, 8, 311-316. 


\section{CHAPTER 6: LOCALIZED SURFACE PLASMON-MEDIATED ENERGY TRANSFER BETWEEN QUANTUM DOTS AND GOLD}

NANOPARTICLES

\subsection{Introduction}

Energy transfer in nanoscale donor-acceptor systems has extensive applications in sensors, bio-imaging, photovoltaic devices, light emitters and other optoelectronics [1-5]. As mentioned in Chapter 2, resonance energy transfer (RET) is one of commonly used and effective strategies in current engineering fields. Förster resonance energy transfer (also called as fluorescence resonance energy transfer, FRET) is a typical nonradiative energy transfer process from an electronic excited molecular donor to a molecular acceptor [6-10]. FRET occurs without the appearance of a photon, and is not the result of emission from the donor being absorbed by the acceptor. Therefore, FRET does not involve emission of light by the donor, and the acceptor does not need to be fluorescent as well. In principle, the FRET efficiency strongly depends on the spectral overlap and the separation distance between donors and acceptors. In conventional FRET systems, both the energy donor and the acceptor are made of organic molecules. The energy is transferred from a fluorescent donor to a fluorescent or non-fluorescent acceptor so that the fluorescence intensity of the donor decreases, and the fluorescence intensity of acceptors increases in the case of fluorescent acceptors.

Inorganic quantum dots (QDs) are substituting for organic dyes as the alternative energy donors because of their size-tunable optical properties and potential multiplexing detection 
capability at a low-cost and simple manner [11]. This has resulted in the development of a variety of QD-based FRET sensors with high sensitivity and reliability [6,7,12]. However, FRET-based sensors have limited detectable distance of about $60 \AA$ [13]. In Chapters 3 and 4, we described the effect of localized surface plasmon resonance (LSPR) on the Raman scattering, leading to the SERS process through the interaction between electromagnetic field from LSPR and Raman scattering. Recently, several studies have been focused on the LSPR in the FRET process [14-18]. Reil et al. found that the FRET process strongly depends on the interaction with plasmonic resonances when a metallic nanoparticle is positioned near to the donor-acceptor pair. Acceptor fluorescence increases at the expense of donor fluorescence because the acceptor molecule harvests the donor's near-field energy. Furthermore, by tuning the plasmonic resonance wavelength close to the emission wavelength of the donor, the near-field energy of the donor will be transformed into light at the expense of the FRET transfer rate and other nanoradiative processes. If the plasmonic resonance wavelength approaches to the emission wavelength of the acceptor, the emission intensity of the acceptor is strongly enhanced due to the energy transfer from the donor. Lakowicz et al. investigated the FRET through positioning various sized silver particles between the donor-acceptor pairs [15]. It was shown that the apparent energy transfer distance increases with the increase of particle size and the distance from the metal core. Theoretical investigation indicates that the RET process is highly sensitive to molecules' position, which is attributed to the plasmon-enhanced radiative transfer rather than by a nonradiative transfer mechanism [16].

Interestingly, gold nanoparticles were also explored as the efficient energy acceptors to substitute for organic acceptors. It has been found that ultra-fine gold nanoparticles $(\sim 3 \mathrm{~nm})$ are able to quench the fluorescent emission of the energy donor in much longer separation distance, 
following $1 / d^{4}$ distance dependence [19-23]. However, most of the previous studies have been focused on ultra-fine gold nanoparticles as acceptors, which have no or negligible LSPR absorption. The QD-gold nanoparticle systems, a representative of semiconductor-metal hybrid nanostructures, have received increasing attention because both QDs and gold nanoparticles can be tailored in size, shape and microstructure, which provides unprecedented flexibility in controlling the electronic and optical properties. In QD-gold nanoparticle systems, the energy quenching efficiency depends on several factors such as the particle size, shape and the separation distance between the donor and the gold quencher [24-30]. Few experiments have been performed to study the effect of size of gold nanoparticles on the energy transfer efficiency and mechanism [15]. The effect of the size-dependent LSPR on the energy transfer efficiency remains unclear.

Understanding of the energy transfer mechanism in QD-metal nanoparticles systems is essential to the construction of devices based on energy transfer. In the present work, three different sized gold nanoparticles $(3 \mathrm{~nm}, 15 \mathrm{~nm}$ and $80 \mathrm{~nm}$ ) that have different LSPR absorption features are selected. The effect of the nanoparticle size on the energy transfer between the $\mathrm{CdSe} / \mathrm{ZnS}$ QDs and the gold nanoparticles will be investigated. The effect of LSPR on the energy transfer efficiency and mechanism will be discussed.

\subsection{Materials and experimental methods}

\subsubsection{Materials}

Cadmium oxide (CdO, 99.99+\%), zinc oxide ( $\mathrm{ZnO}, 99.99 \%$ ), sulfur (powder, 99.98\%), trioctylphosphine oxide (TOPO, tech. 90\%), 1-octadecene (ODE, tech. 90\%), oleic acid (tech. 90\%), octyldecylamine (ODA, tech. 90\%), trioctylphosphine (TOP, tech. 90\%), selenium 
(powder, 99+\%), 3-mercaptopropionic acid (MPA, 99+\%), $\mathrm{Hg}\left(\mathrm{NO}_{3}\right)_{2}$ and $5 \mathrm{M} \mathrm{NaCl}$ stock solution were purchased from Sigma-Aldrich. n-tetradecylphosphonic acid (TDPA) was purchased from PCI Synthesis. Methylene dichloride $\left(\mathrm{CH}_{2} \mathrm{Cl}_{2}\right.$, HPLC grade), methanol (ACS grade) and acetone (ACS grade) were purchased from Fisher Scientific. Chloroauric acid trihydrate $\left(\mathrm{HAuCl}_{4} \cdot 3 \mathrm{H}_{2} \mathrm{O}\right), \mathrm{NaBH}_{4}(98 \%)$ and ethylenediamine $(99 \%)$ were obtained from AlfaAesar. D.I. water was obtained from a Milli-Q academic Millipore system. All chemicals and solvents were obtained from the commercial sources and used directly without any further purification, and all glassware were cleaned successively with $\mathrm{HNO}_{3}$ and D.I. water, and then dried before use.

\subsubsection{Synthesis of CdSe/ZnS QDs}

CdSe/ZnS QDs were synthesized by a well-established method with slight modification [31,32]. CdO (0.4 mmol), TDPA (0.2 g), ODA (1.0 g) and TOPO (2.5 g) were added into a 50 $\mathrm{mL}$ three-neck flask and were evacuated for $30 \mathrm{~min}$ at $100{ }^{\circ} \mathrm{C}$. The mixture was then heated to 300-320 ${ }^{\circ} \mathrm{C}$ under Ar-flow to obtain a colorless clear solution. After that, the temperature was lowered to $260-270{ }^{\circ} \mathrm{C}$ for the injection of TOPSe (0.03 g Se in $2.4 \mathrm{~mL}$ TOP). Following this, the temperature was lowered to $240{ }^{\circ} \mathrm{C}$ for $\mathrm{CdSe}$ growth and the fluorescence emission was monitored using the $365 \mathrm{~nm}$ UV light. Once the desired fluorescence emission was observed, the temperature was lowered to about $220{ }^{\circ} \mathrm{C}$, and the calculated amount of the $\mathrm{Zn}$ - or S- stock solution $(0.407 \mathrm{~g} \mathrm{ZnO}$ in $20 \mathrm{~mL}$ oleic acid and $30 \mathrm{~mL}$ ODE for $0.1 \mathrm{M}$ Zn-stock solution, and $0.16 \mathrm{~g} \mathrm{~S}$ in $50 \mathrm{~mL}$ ODE for $0.1 \mathrm{M} \mathrm{S}$-stock solution) for each $\mathrm{ZnS}$ monolayer was alternately injected. The CdSe/ZnS QDs with 2 ZnS monolayers were obtained. 


\subsubsection{Surface modification of CdSe/ZnS QDs with MPA}

The as-made QDs were coated by the hydrophobic ligands, TOPO and ODA, and thus the asmade QDs cannot dissolve in the aqueous solution. The water soluble MPA-QDs were prepared through the ligand exchange method [33-35]. Firstly, the MPA-KOH methanolic stock solution was obtained by adding $4 \mathrm{~mL}$ MPA and $3.0 \mathrm{~g} \mathrm{KOH}$ into $40 \mathrm{~mL}$ methanol. $5 \mathrm{~mL}$ TOPO-capped QDs in $\mathrm{CH}_{2} \mathrm{Cl}_{2}$ with the optical density (O.D.) of 1.9 were precipitated and purified 4 times by addition of methanol and acetone, and redispersed in $1 \mathrm{~mL} \mathrm{CH}_{2} \mathrm{Cl}_{2}$, followed by the centrifugation at a speed of 3,000 rpm to remove impurities. $200 \mu \mathrm{L}$ of MPA-KOH methanolic stock solution was added and incubated overnight. The precipitates can be observed, indicating the formation of the hydrophilic QDs. The suspension was centrifuged at a speed of 3,000 rpm and washed 4 times by $\mathrm{CH}_{2} \mathrm{Cl}_{2}$ and methanol to remove the excess MPA and exchanged organic ligands. The resulting precipitates were dispersed into $20 \mathrm{~mL} 0.3 \mathrm{M}$ PBS $(0.3 \mathrm{M} \mathrm{NaCl}, 10 \mathrm{mM}$ $\mathrm{NaH}_{2} \mathrm{PO}_{4} / \mathrm{Na}_{2} \mathrm{HPO}_{4}$ buffer solution with $\mathrm{pH}=7$ ), and then centrifuged at a very low speed to obtain a clear water soluble MPA-QDs for further use.

\subsubsection{Synthesis of DNA-modified CdSe/ZnS QDs and gold nanoparticles}

Gold nanoparticles with different sizes were synthesized through the reduction of $\mathrm{HAuCl}_{4} \cdot 3 \mathrm{H}_{2} \mathrm{O}$ by $\mathrm{NaBH}_{4}$ or trisodium citrate, as described in Section 3.2 [36-38]. The $80 \mathrm{~nm}$ gold nanoparticles were provided by a commercial source (BioAssay Works Naked Gold, USA). The ssDNAs were synthesized, purified and deprotected according to the well-documented protocol [39]. Functionalization of QDs and gold nanoparticles with DNA was carried out according to the well-established procedures by Mirkin [40] and Nie groups [41]. The watersoluble QDs and gold nanoparticles were attached by synthetic ssDNA with a $\mathrm{C}_{6}$ spacer 
appended to the 5'-phosphate backbone terminus of a $10 \mathrm{bp}$ (base pair), $20 \mathrm{bp}$ and $30 \mathrm{bp}$. The amount of the loading DNA was controlled to at most 1 ssDNA molecule per particle, based on the UV-vis absorption spectra. The spectra of the solution before and after conjugation were recorded, and the loading of ssDNA per particle was calculated from the absorbance difference at $260 \mathrm{~nm}$ based on 1 O.D. $(260 \mathrm{~nm})=50 \mathrm{ng} \mathrm{DNA} / \mu \mathrm{L}$. Also, the particle concentration was obtained from the UV-vis absorption spectrum based on Beer-Lambert Law using the molar extinction coefficient at the wavelength of maximum absorbance $\left(\varepsilon(\mathrm{QDs}, 570 \mathrm{~nm})=2.0 \times 105 \mathrm{M}^{-}\right.$ ${ }^{1} \cdot \mathrm{cm}^{-1}$ [42], $\varepsilon(3 \mathrm{~nm} \mathrm{Au}, 506 \mathrm{~nm})=1.1 \times 105 \mathrm{M}^{-1} \cdot \mathrm{cm}^{-1}$ [19], $\varepsilon(15 \mathrm{~nm} \mathrm{Au}, 520 \mathrm{~nm})=1.0 \times 108 \mathrm{M}^{-}$ ${ }^{1} \cdot \mathrm{cm}^{-1}[43]$, and $\left.\varepsilon(80 \mathrm{~nm} \mathrm{Au}, 550 \mathrm{~nm})=6.9 \times 1010 \mathrm{M}^{-1} \cdot \mathrm{cm}^{-1}[44]\right)$.

\subsubsection{Gold nanoparticle-QD conjugates}

Gold nanoparticle-QD conjugate ensemble was prepared by the formation of thymidine- $\mathrm{Hg}^{2+}-$ thymidine $\left(\mathrm{T}-\mathrm{Hg}^{2+}-\mathrm{T}\right)$ complexes in the presence of $\mathrm{Hg}^{2+}$, First, the assay solution was made by adding DNA-gold conjugates $(6.0 \mathrm{nM})$ and DNA-QD conjugates $(6.0 \mathrm{nM})$ into the $0.3 \mathrm{M}$ PBS solution containing $0.3 \mathrm{M} \mathrm{NaCl}, 10 \mathrm{mM} \mathrm{Na} 2 \mathrm{HPO}_{4} / \mathrm{NaH}_{2} \mathrm{PO}_{4}$ and $0.1 \mathrm{mM}$ ethylenediamine. Following this, the quenching assay was carried out by adding various concentrations of $\mathrm{Hg}^{2+}$ into the assay solution. The fluorescence intensity at $570 \mathrm{~nm}$ was monitored.

\subsubsection{Characterization}

The size and morphology of the nanoparticles were observed with a JEM 2100F transmission electron microscope (TEM) with an acceleration voltage of $200 \mathrm{kV}$. UV-visible absorption spectra were measured in the range of 200-800 nm with a Shimadzu UV-2550 spectrometer. The fluorescence (FL) emission spectra were measured using a Hitachi F-7000 fluorescence 
spectrophotometer. The FL quantum yield (QY) was determined using Rhodamine $6 \mathrm{G}$ as a reference.

\subsection{Characterization and size-tunable optical properties of QDs}

Semiconducting nanocrystals synthesized by colloidal chemistry techniques provide unprecedented flexibility toward control of size-tunable optical properties. Optical spectra (including absorption spectra and emission spectra) of CdSe/ZnS QDs can be tuned from the blue emission region $(\sim 450 \mathrm{~nm})$ to the red emission region $(\sim 650 \mathrm{~nm})$ through adjusting the reaction conditions (temperature, time or recipe) [45-47]. The classical organometallic route uses highly toxic, expensive and pyroforic $\mathrm{Cd}\left(\mathrm{CH}_{3}\right)_{2}$ and bis(trimethylsilyl) sulfide as reagents [48]. However, novel greener synthetic schemes using environmentally more benign cadmium precursor $\mathrm{CdO}$ is replacing the classical synthetic route $[46,49,50]$. We used $\mathrm{CdO}$ and $\mathrm{Se}$ as raw materials to synthesize CdSe, and following this, the $\mathrm{ZnS}$ shell was grown using successive ion layer adsorption and reaction method. A series of QDs with various fluorescence emission wavelengths were obtained. Figure 6.1 shows optical absorption spectra and fluorescence emission spectra of representative $\mathrm{CdSe} / \mathrm{ZnS}$ core/shell QDs with various sizes. It was found that optical absorption and fluorescence emission spectra red-shift with the increasing reaction time. We can tune the fluorescence emission wavelength from $510 \mathrm{~nm}$ to $620 \mathrm{~nm}$ in the present work. Figure 6.1c shows optical photos of representative QDs under excitation of $365 \mathrm{~nm}$ wavelength. 

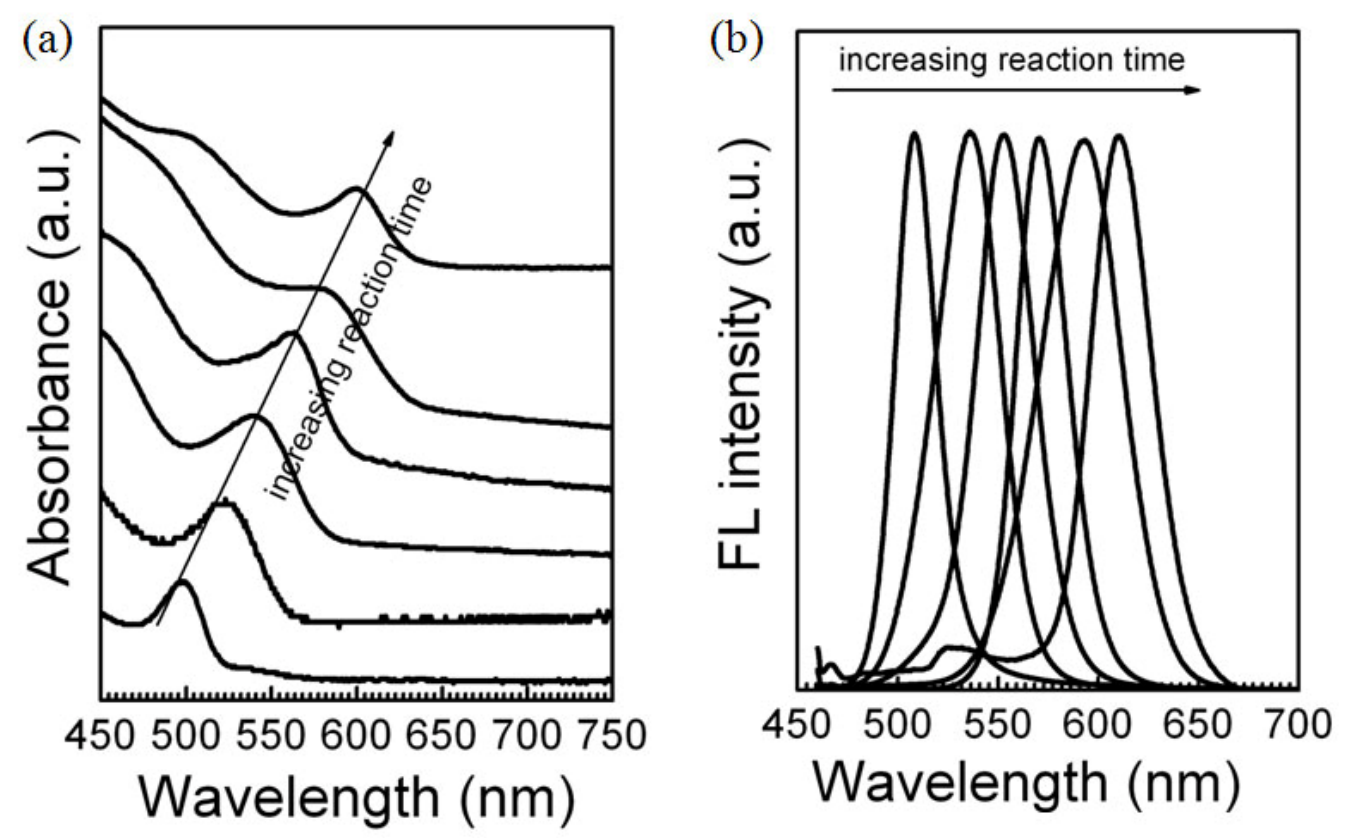

(c)

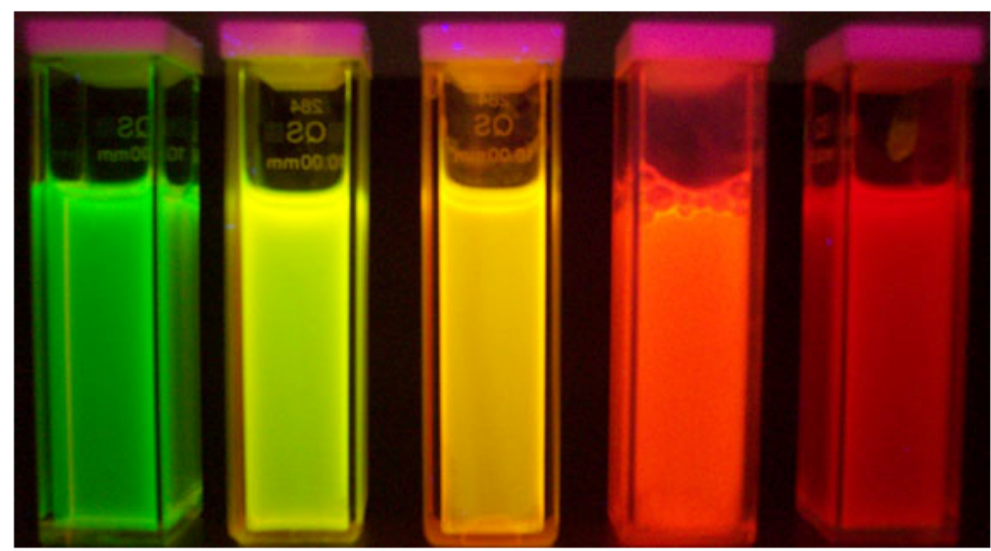

Figure 6.1 (a) Optical absorption spectra and (b) fluorescence emission spectra of representative $\mathrm{CdSe} / \mathrm{ZnS}$ core/shell quantum dots dispersed in $\mathrm{CH}_{2} \mathrm{Cl}_{2}$. (c) Optical photos of representative $\mathrm{CdSe} / \mathrm{ZnS}$ core/shell quantum dots in $\mathrm{CH}_{2} \mathrm{Cl}_{2}$ under $365 \mathrm{~nm}$ excitation.

Figure 6.2a shows the representative TEM image of CdSe/ZnS QDs with the fluorescence emission of $570 \mathrm{~nm}$. It is clearly seen that QDs exhibit excellent dispersivity in $\mathrm{CH}_{2} \mathrm{Cl}_{2}$, and possess a uniform size distribution with the size of $3.4 \mathrm{~nm}$ in diameter. The high resolution TEM 
image demonstrates the high crystallinity of the present QDs. In one word, the above results show the controllable synthesis of QDs in the present work.
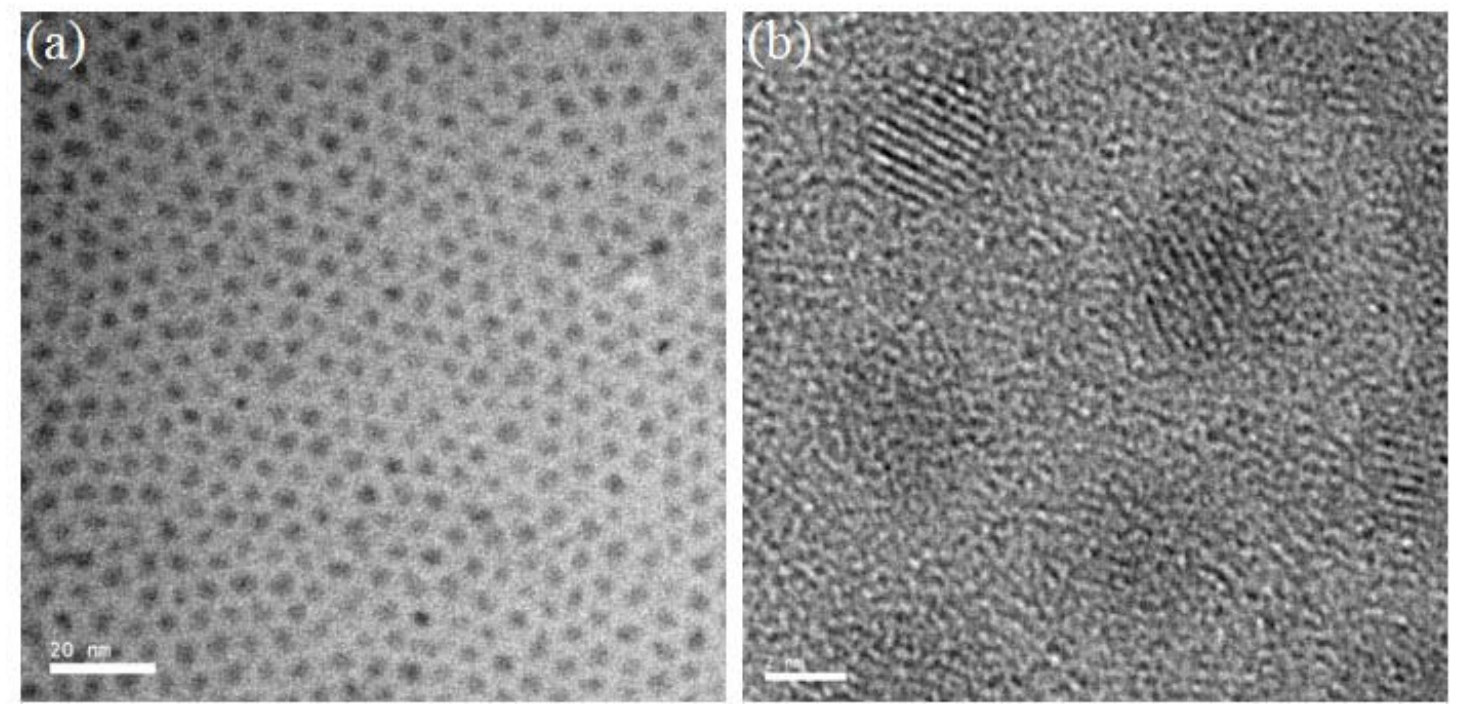

Figure 6.2 (a) TEM image (scale bar: $20 \mathrm{~nm}$ ) and (b) HRTEM image (scale bar: $2 \mathrm{~nm}$ ) of $\mathrm{CdSe} / \mathrm{ZnS}$ core/shell quantum dots with the fluorescence emission wavelength of $570 \mathrm{~nm}$.

Furthermore, we checked the chemistry composition and surface chemistry using XPS and FTIR. XPS shows that the prepared sample contains $\mathrm{P}$ and $\mathrm{N}$ elements in addition to $\mathrm{Cd}, \mathrm{Se}, \mathrm{Zn}$ and S, which indicates the surface chemistry with TOPO and ODA ligands on the QD surface (Figure 6.3). In the FTIR spectrum (Figure 6.4), peaks at $2921 \mathrm{~cm}^{-1}$ and $2847 \mathrm{~cm}^{-1}$ are assigned to the asymmetric and symmetric stretching vibration modes of $\mathrm{C}-\mathrm{H}$, respectively [51]. The 1638 $\mathrm{cm}^{-1}$ peak is assigned to the $\mathrm{C}=\mathrm{C}$ stretching mode from the ODA ligand [52], and the $1467 \mathrm{~cm}^{-}$ ${ }^{1}$ peak and the broad band around $1086 \mathrm{~cm}^{-1} \sim 1005 \mathrm{~cm}^{-1}$ are attributed to the deformation mode of $-\mathrm{CH}_{3}$ and the stretching mode of C-C, respectively [53]. Therefore, XPS and FTIR demonstrate 
the complicated surface chemistry on the prepared QDs, which makes QDs soluble in hydrophobic organic solvents (e.g. $\mathrm{CH}_{2} \mathrm{Cl}_{2}$ and toluene).

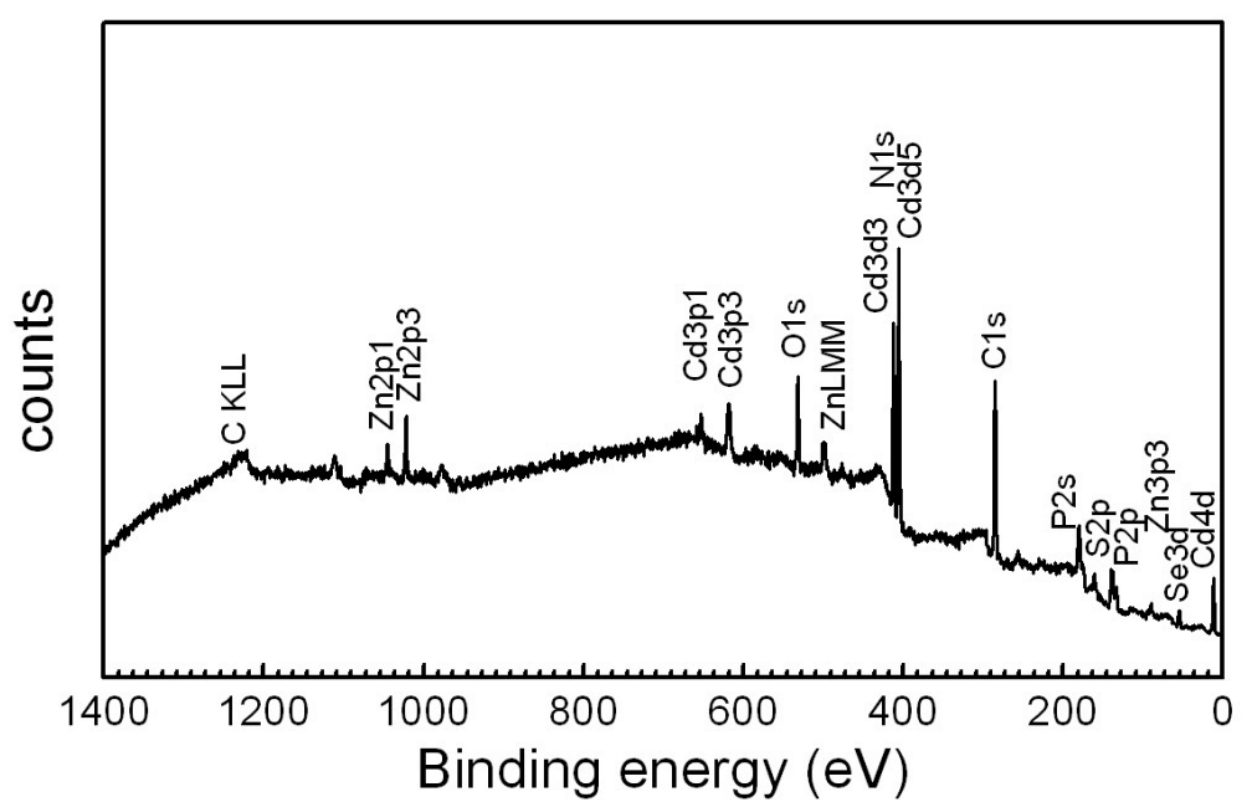

Figure 6.3 XPS survey scan of $\mathrm{CdSe} / \mathrm{ZnS}$ core/shell quantum dots with the fluorescence emission wavelength of $570 \mathrm{~nm}$.

\subsection{Preparation of water-soluble QDs and its surface functionalization}

The prepared CdSe/ZnS QDs are hydrophobic in the present work, and its surface is capped by hydrophobic ligands, like TOPO and ODA. Nevertheless, the biological applications require QDs water-soluble so that many of studies are focused on the preparation of water-soluble QDs $[35,54,55]$. Currently, the commonly used strategy for enabling biocompatibility is to modify the surface of QDs with hydrophilic ligands, like 3-mercaptobenzoic acid (MPA), dihydrolipoic acid (DHLA), HS-poly(ethylene glycol)-carboxyl acid (HS-PEG-COOH). In the present work, we 
employed the ligand exchange method to make the water-soluble QDs capped by MPA (Figure 6.5). The optical photo shows the excellent water solubility in PBS.

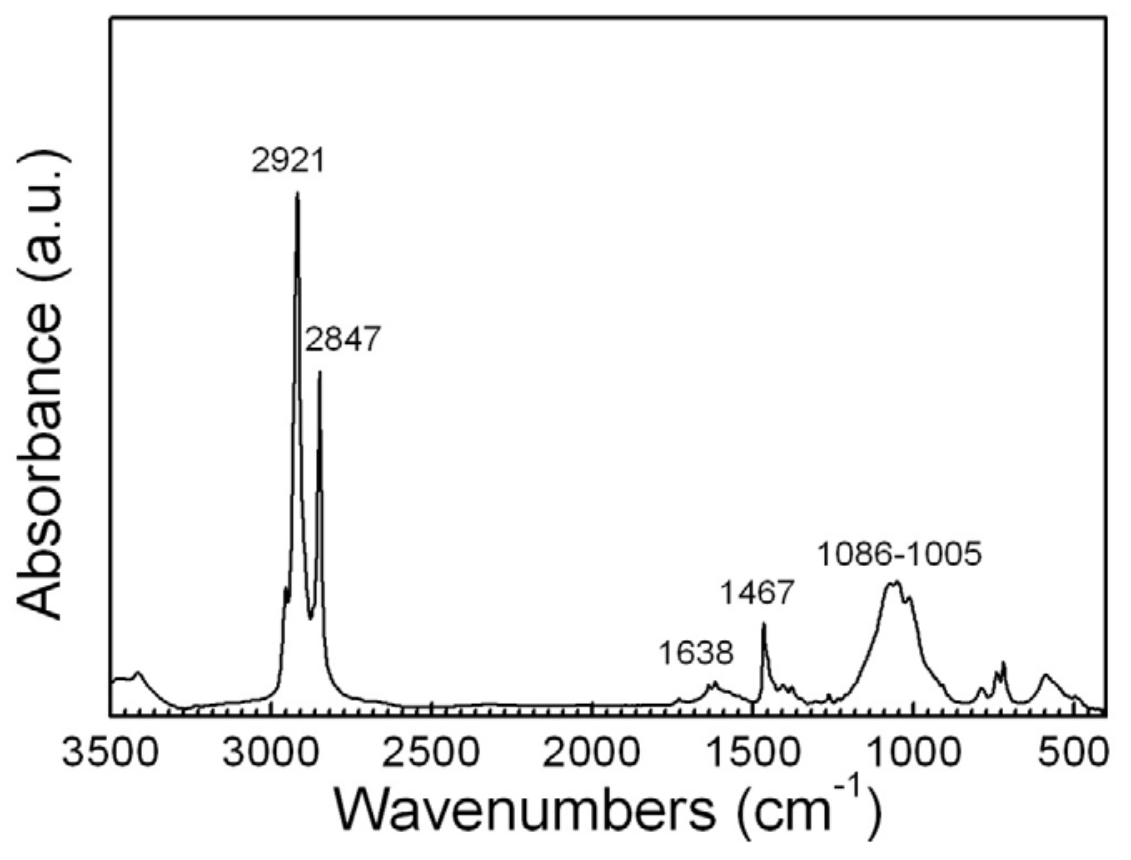

Figure 6.4 FTIR of $\mathrm{CdSe} / \mathrm{ZnS}$ core/shell quantum dots with the fluorescence emission wavelength of $570 \mathrm{~nm}$.
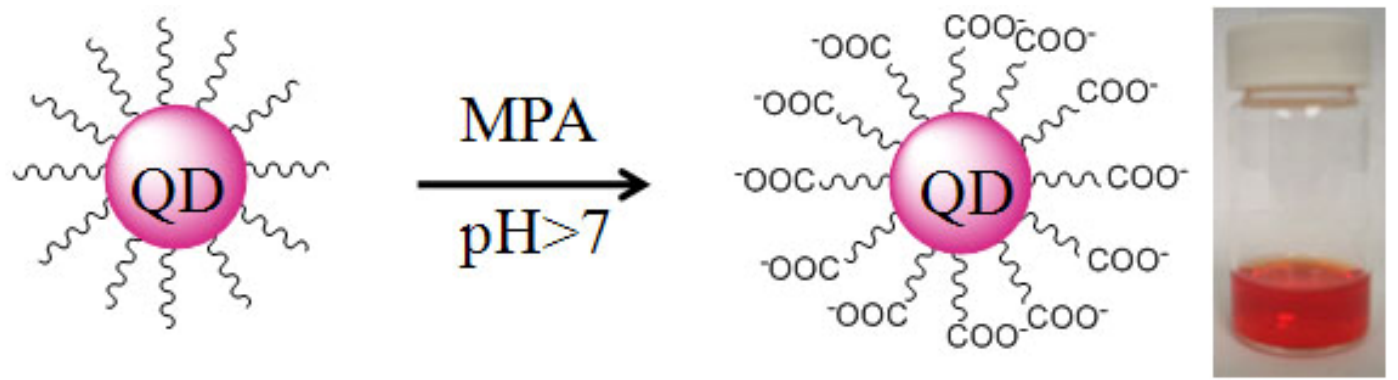

Figure 6.5 Water-soluble CdSe/ZnS quantum dots through the ligand exchange method. Optical photo shows the excellent water solubility in PBS. 

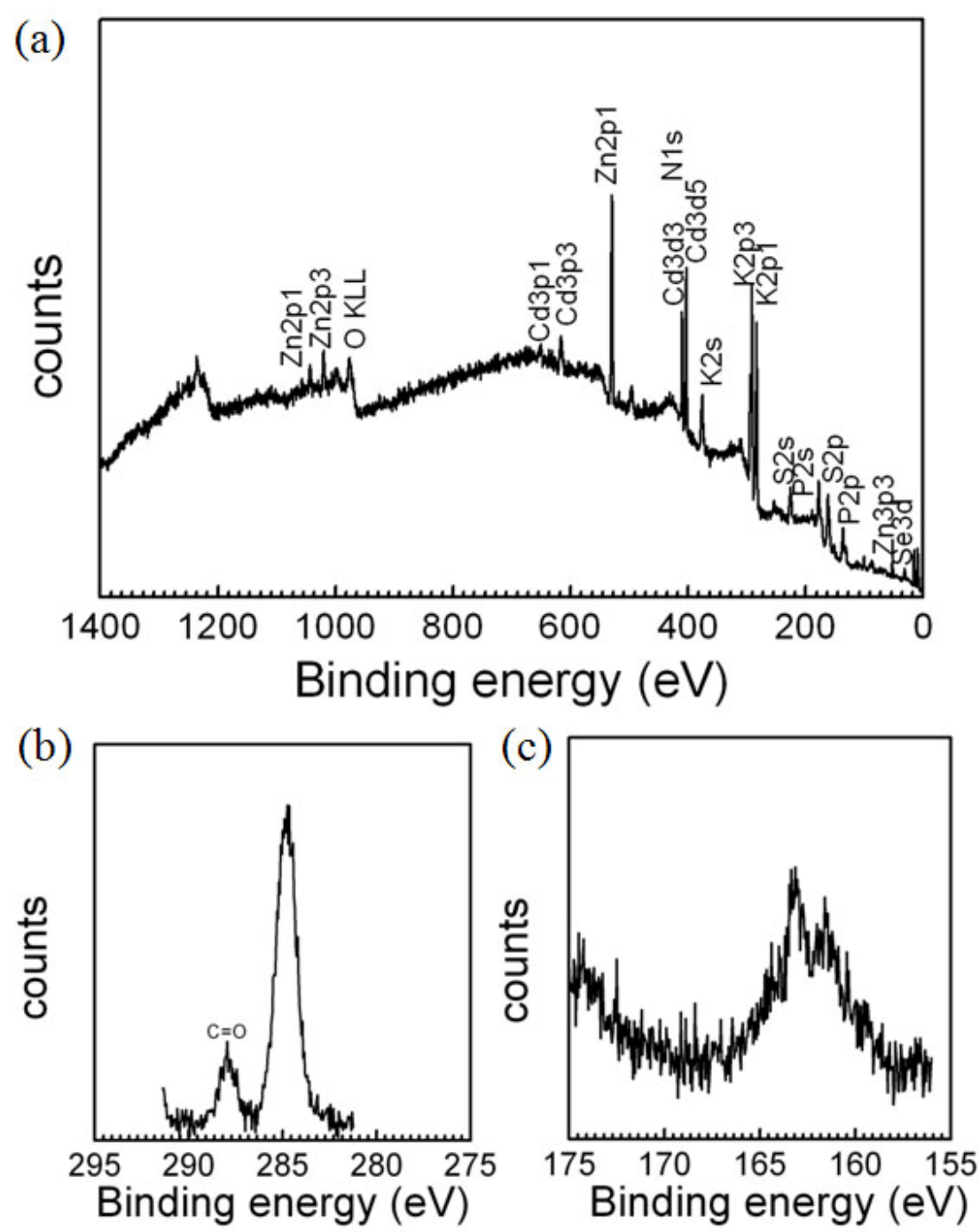

Figure 6.6 XPS (a) survey scan, (b) C1s core level and (c) S2p core level of 3mercaptopropionic acid-modified $\mathrm{CdSe} / \mathrm{ZnS}$ quantum dots.

XPS survey scan shows that MPA-modified QDs have a similar elemental composition with the TOPO/ODA-capped QDs (Figures 6.5 and 6.6), except that MPA-modified QDs contain K element. This is because of $\mathrm{KOH}$ residues from the raw material MPA-KOH. In addition, it can be clearly seen that the $\mathrm{C} 1 \mathrm{~s}$ core level shows the obvious $288.8 \mathrm{eV}$ peak, assigned to the $\mathrm{C}=\mathrm{O}$ 
[51]. FTIR spectrum of MPA-modified QDs exhibits a broad shoulder around $1680 \mathrm{~cm}^{-1}$ and a sharp peak at $1576 \mathrm{~cm}^{-1}$ (Figure 6.7), which are attributed to the vibrational mode of free $\mathrm{COOH}$ and the vibration of $\mathrm{COO}^{-}$anion [56]. Thus, we demonstrate the successful surface modification of QDs using the ligand exchange method.

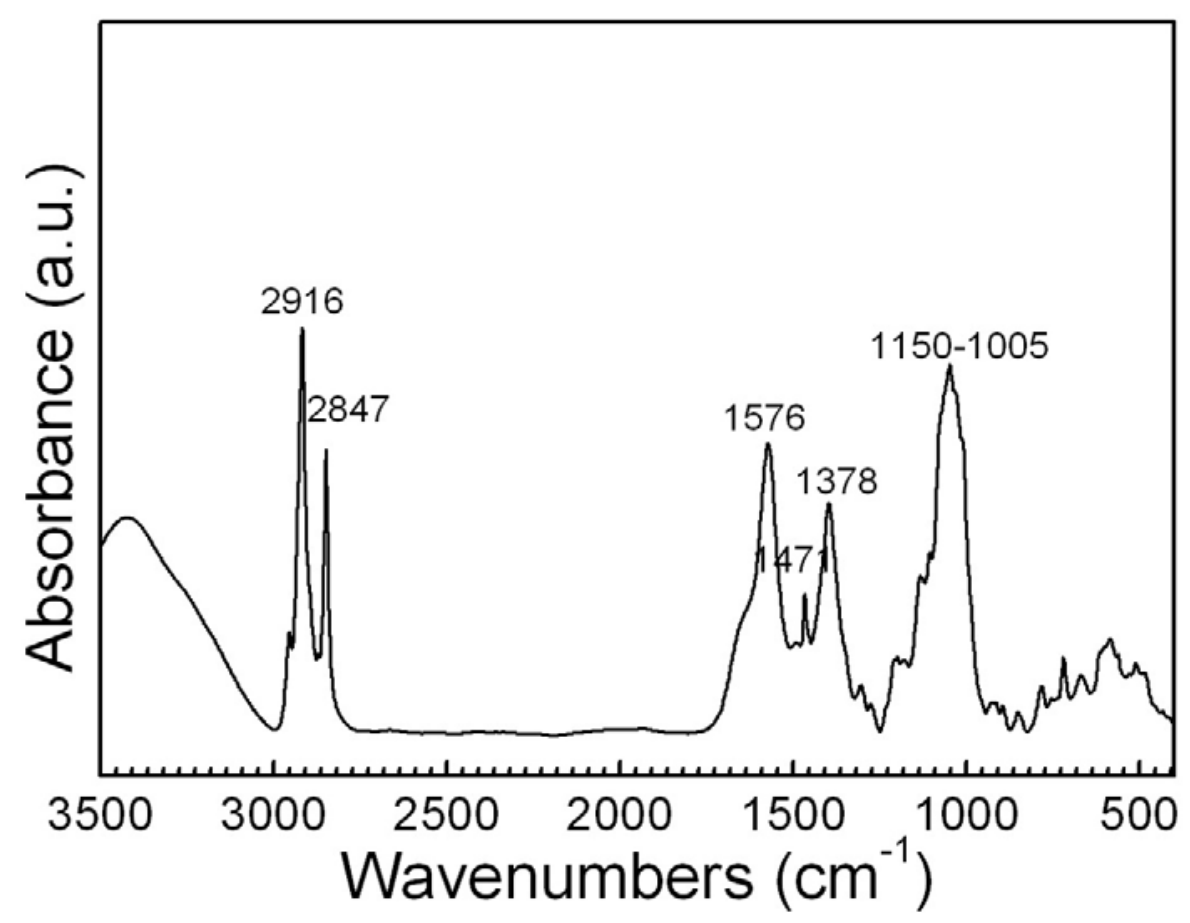

Figure 6.7 FT-IR spectra of 3-mercaptopropionic acid-modified CdSe/ZnS quantum dots.

\subsection{Design and assembly of energy transfer system}

Figure 6.8 schematically shows the nanoscale energy transfer system in which the QD acts as the energy donor, the gold nanoparticle as the energy acceptor and the rigid double-stranded DNA as the spacer between the QD and the gold nanoparticle. The separation distance between the QD and the gold nanoparticle is controlled by the length of DNA, which are shown in Figure 
6.8. QDs with $3.4 \mathrm{~nm}$ in diameter and the fluorescence emission at $570 \mathrm{~nm}$ were used as the energy donor. Gold nanoparticles with different particles sizes are used as acceptors. The TEM images in Figure 6.9 reveal the size distribution of the $3 \mathrm{~nm}, 15 \mathrm{~nm}$ and $80 \mathrm{~nm}$ sized gold nanoparticles (Figure 6.9b-d). It can be seen from the UV-visible absorption spectra that there was no observable LSPR absorption for the $3 \mathrm{~nm}$ gold nanoparticles (Figure 6.9a) [57]. In contrast, the $15 \mathrm{~nm}$ and $80 \mathrm{~nm}$ gold nanoparticles showed strong LSPR band at $520 \mathrm{~nm}$ and 550 $\mathrm{nm}$, respectively (Figure 6.9a). The fluorescence emission band of the QDs exhibited partial spectral overlap with the LSPR band of the $15 \mathrm{~nm}$ gold nanoparticles, and complete overlap with the LSPR band of $80 \mathrm{~nm}$ gold nanoparticles (Figure 6.9a).
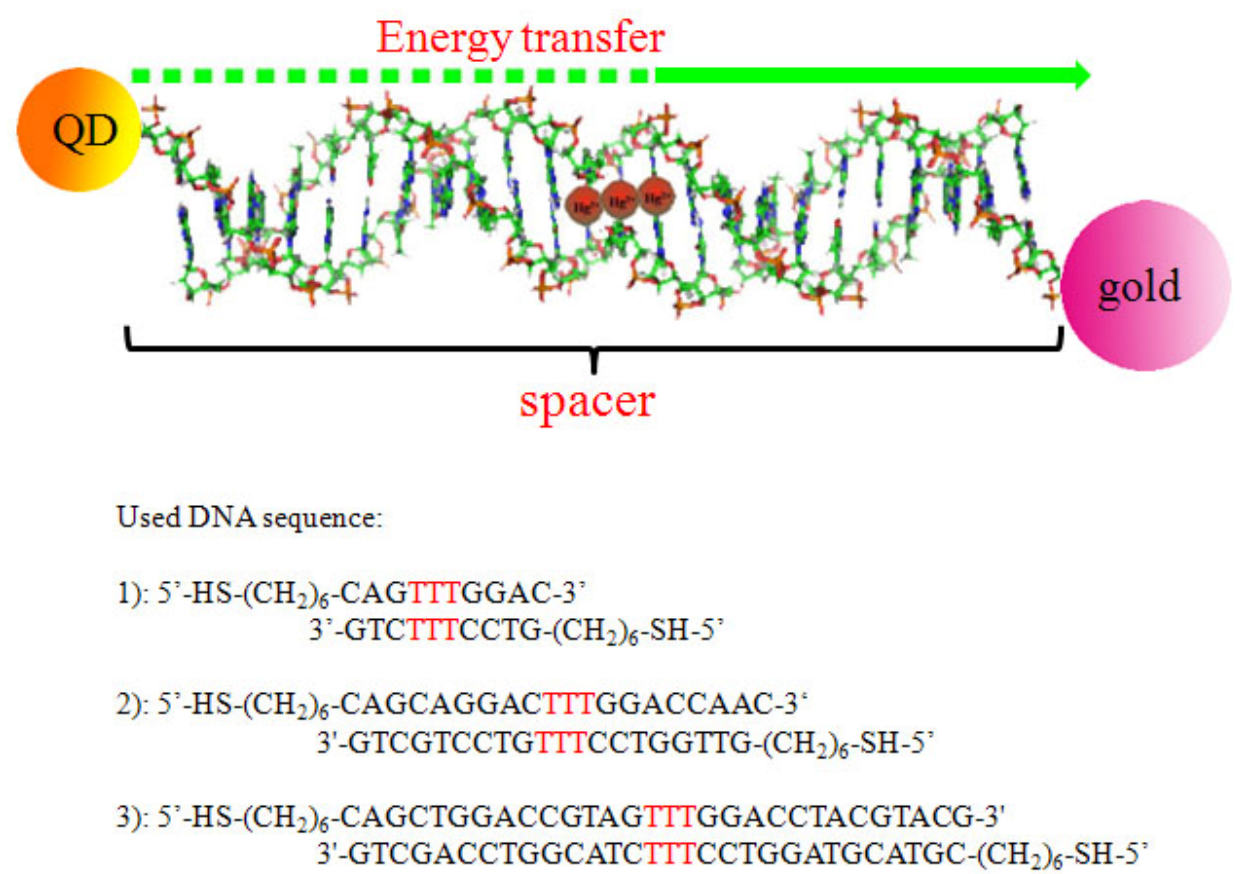

Figure 6.8 Schematic illustration of the quantum dot (energy donor)-DNA-gold nanoparticle (acceptor) hybrid system in the presence of $\mathrm{Hg}(\mathrm{II})$ ions and used DNA sequences. 
(a)

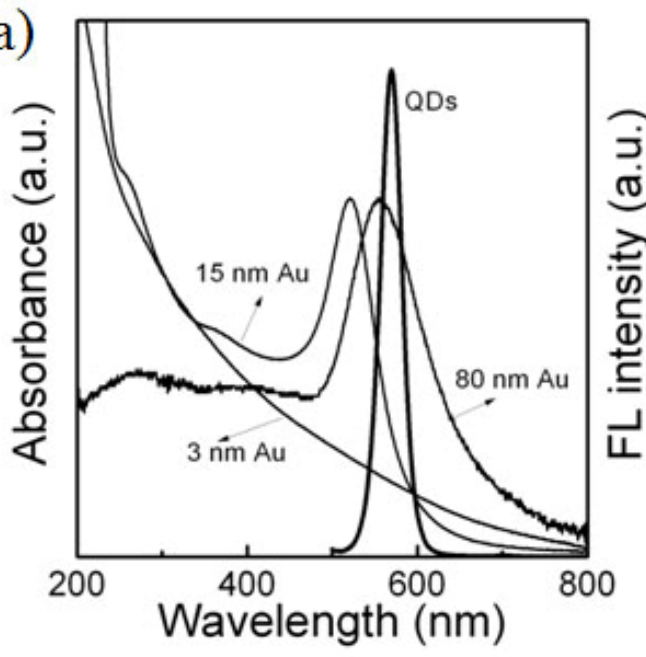

(c)

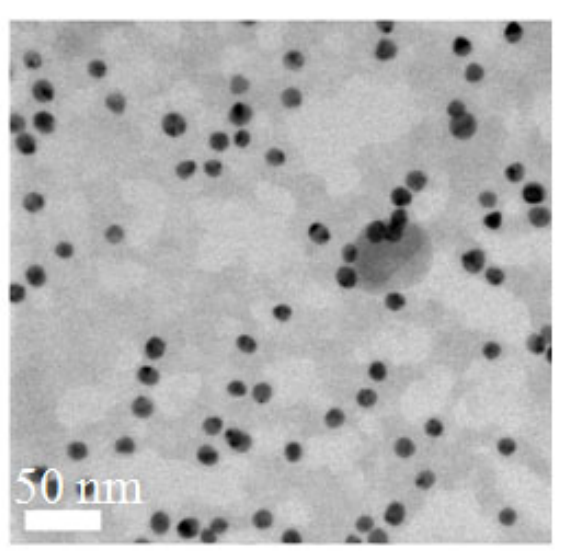

(b)

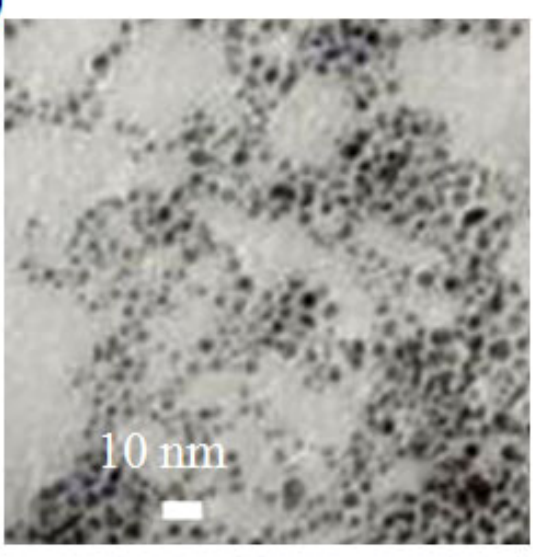

(d)

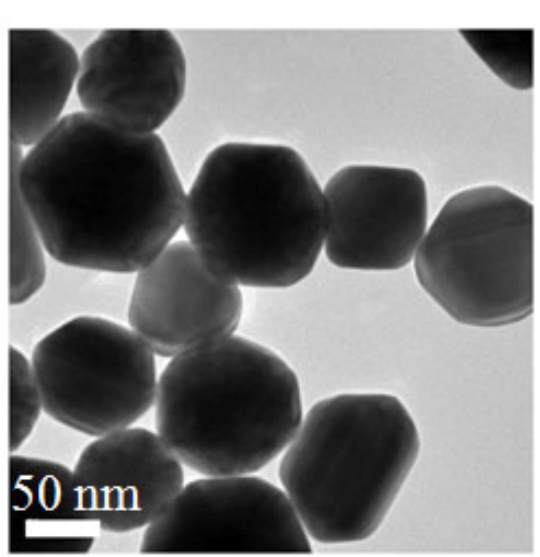

Figure 6.9 (a) Extinction spectra of $3 \mathrm{~nm}, 15 \mathrm{~nm}$ and $80 \mathrm{~nm}$ gold nanoparticles, and fluorescence emission spectra of DNA-functionalized quantum dots. (b-d) TEM images of $3 \mathrm{~nm}, 15 \mathrm{~nm}$ and $80 \mathrm{~nm}$ gold nanoparticles, respectively.

The ssDNA molecules attached to the QDs and to the gold nanoparticles were complementary except for three deliberately designed T-T mismatches in the middle of the DNA strands (Figure 6.8). The absorption spectra indicate that the average loading of ssDNA was less than one DNA molecule per QD or per gold nanoparticle [58]. In the absence of $\mathrm{Hg}^{2+}$ in the aqueous solution, the free-standing ssDNA-QDs and the ssDNA-gold nanoparticles were well dispersed and unable 
to hybridize together due to the mismatched T-T base pairs. The fluorescence emission at 570 $\mathrm{nm}$ under laser excitation was observed. When $\mathrm{Hg}^{2+}$ was added into the solution, DNA hybridization occurred due to the formation of $\mathrm{T}-\mathrm{Hg}^{2+}-\mathrm{T}$ complexes. As a result, the QDs and the gold nanoparticles were brought into close proximity, which enabled the energy transfer from the QDs to the gold nanoparticles, leading to quenching of the fluorescence emission of the QDs $[13,23,58]$. Firstly, we carried out a control experiment where $\mathrm{Hg}^{2+}$ was added to a solution containing only ssDNA-QDs in the absence of the ssDNA-gold nanoparticles, and there is no observable change in the intensity of the fluorescence emission of the QDs (Figure 6.10). This suggests that fluorescence emission was quenched by the gold nanoparticles rather than the $\mathrm{Hg}^{2+}$ ions $[59,60]$. Willner and co-workers found that the $\mathrm{Hg}^{2+}$ can quench the fluorescence emission of QDs through the electron transfer path [59]. However, this is not the case in our present work because the electron transfer occurs only in the duplex DNA structure [60]. In Willner's work, addition of $\mathrm{Hg}^{2+}$ can induce the sandwich structure of DNA and thus results in the duplex structure while our present DNA sequences do not enable this process in the absence of the complementary DNA-gold nanoparticles and $\mathrm{Hg}^{2+}$. Therefore, the electron transfer quenching cannot explain the quenching of fluorescence emission of QDs in the present system. 

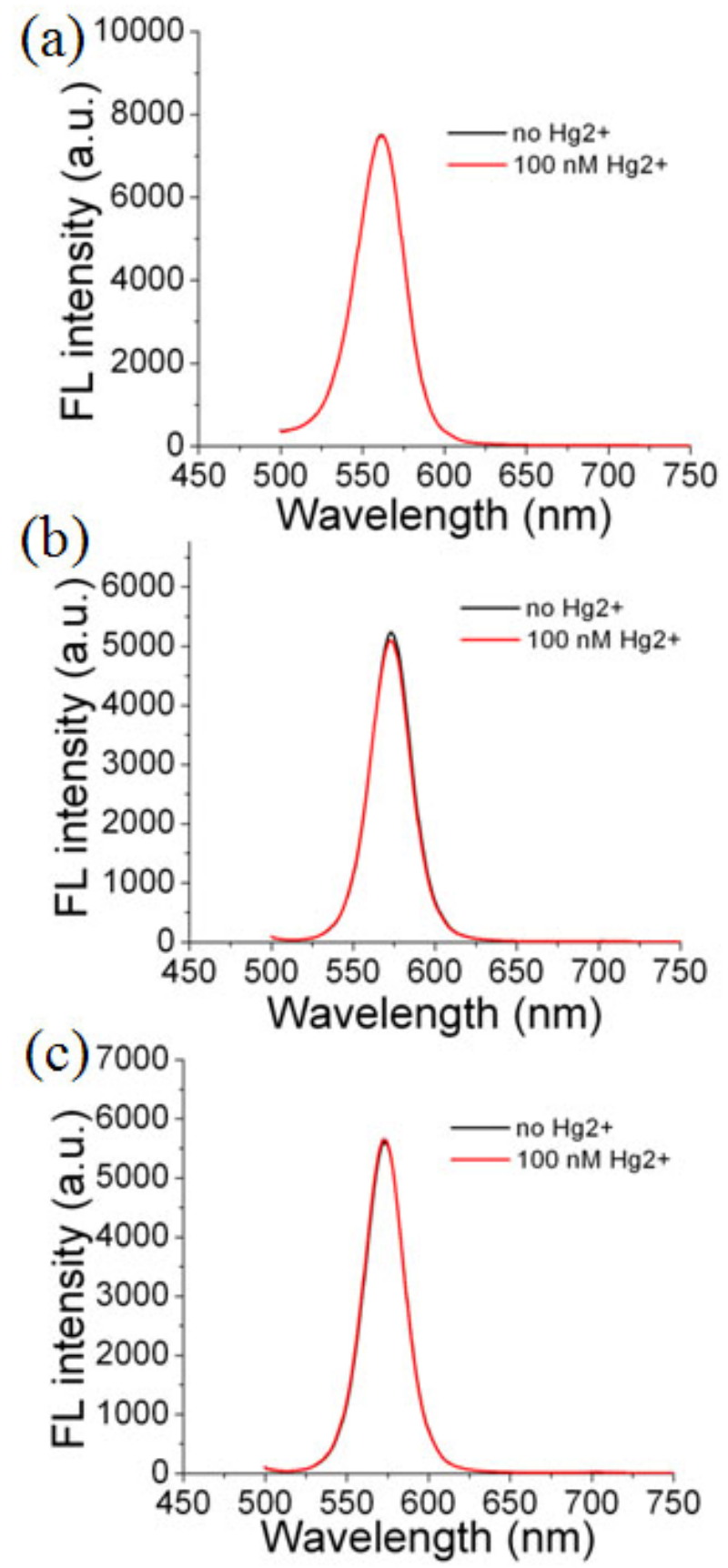

Figure 6.10 Fluorescence spectra of the solution with (a) 10 base DNA-, (b) 20 base DNA- and (c) 30 base DNA-functionalized QDs without gold nanoparticles in the absence and presence of $100 \mathrm{nM} \mathrm{Hg}^{2+}$. The FL intensity shows no observable change after addition of $100 \mathrm{nM} \mathrm{Hg}^{2+}$, 
indicating that $\mathrm{Hg}^{2+}$ makes negligible contribution to quenching of fluorescence from QDs in the present work.

\subsection{Size-dependent fluorescence quenching of QDs}

Figure 6.11a shows the resulting fluorescence emission spectra of the QD/DNA/gold system following successive addition of $\mathrm{Hg}^{2+}$. The fluorescence emission intensity decreased gradually with an increase in the $\mathrm{Hg}^{2+}$ concentration for all three different sized gold nanoparticles (Figure 6.11b). The fluorescence quenching efficiency can be quantified by the Stern-Volmer equation [61]

$$
\frac{F_{0}}{F}=1+k \bullet[Q]
$$

where $F_{0}$ and $F$ are the fluorescence intensity in the absence and the presence of the quencher (gold nanoparticle), respectively; $k$ is the quenching constant, and $Q$ is the concentration of quencher. In the present work, the gold nanoparticles quenched the fluorescence emission only when $\mathrm{Hg}^{2+}$ induced the DNA hybridization. Hence it is reasonable to replace the term $[Q]$ with the $\mathrm{Hg}^{2+}$ concentration. The Stern-Volmer plots in Figure $6.11 \mathrm{c}$ show that the quenching efficiency increased with the size of the gold particles. The quenching constants derived from the slope in Figure $6.11 \mathrm{c}$ were $1.2 \times 10^{7} \mathrm{M}^{-1}, 5.5 \times 10^{7} \mathrm{M}^{-1}$ and $2.6 \times 10^{8} \mathrm{M}^{-1}$ for the $3 \mathrm{~nm}, 15 \mathrm{~nm}$ and $80 \mathrm{~nm}$ gold nanoparticles, respectively.

When the gold nanoparticles are large (such as $15 \mathrm{~nm}$ and $80 \mathrm{~nm}$ sized gold nanoparticle in the present study), a distinct LSPR peak exists in the UV-visible absorption spectra, and FRET is the dominant energy transfer mechanism for donor-acceptor separation distances investigated. This occurs because of the fact that the dipole of the quantum dot can efficiently couple with the 
dipole of LSPR field near the gold nanoparticle. The dipole-dipole interaction is expected to lead to a $1 / d^{6}$-distance dependence for the quenching efficiency, as is usually seen in the FRET process. A discrepancy in the quenching efficiency still exists between the $15 \mathrm{~nm}$ and $80 \mathrm{~nm}$ nanoparticles. The most likely reason is due to larger spectral overlap between the $80 \mathrm{~nm}$ LSPR absorption of the gold nanoparticles and the emission of the quantum dots. However, the increase in the coulombic attraction with size and the existence of a quadrupole mode in the $80 \mathrm{~nm}$ gold nanoparticle could also have an effect [62].

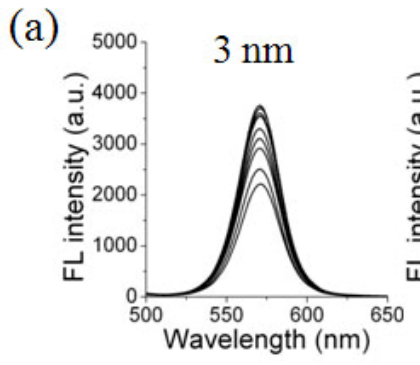

(b)

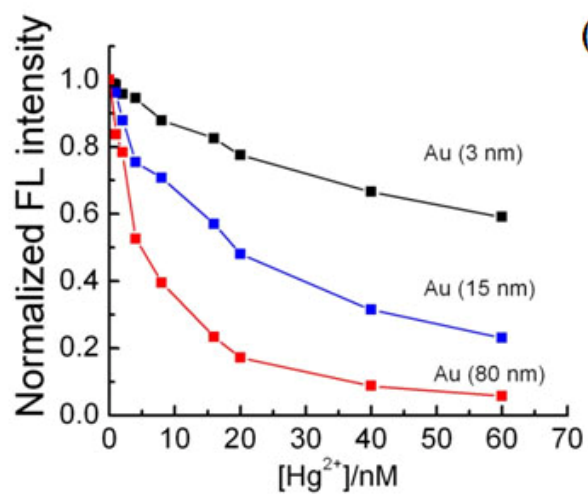

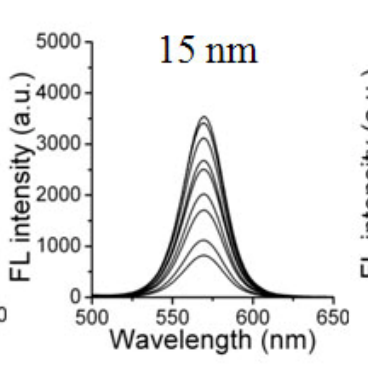
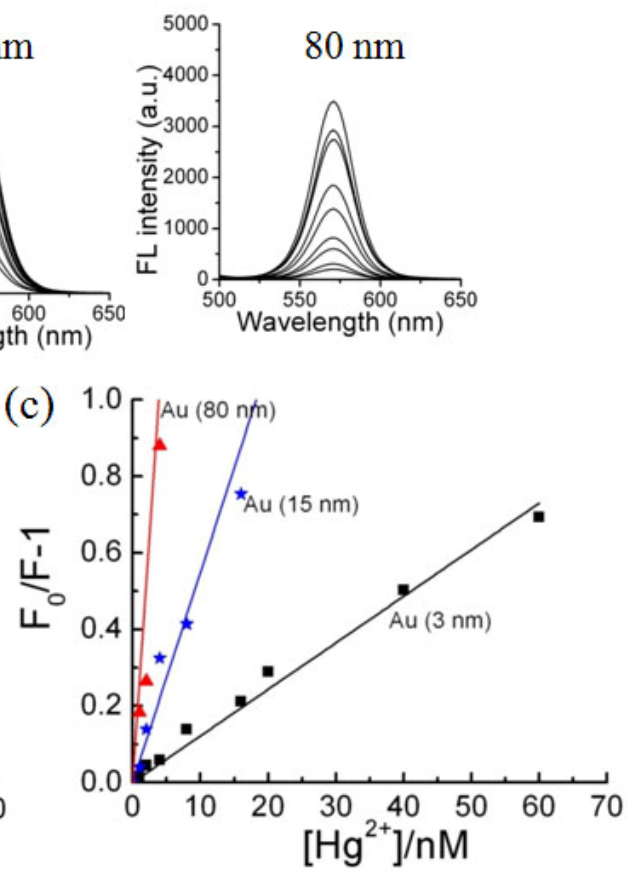

Figure 6.11 Quenching of fluorescence emission of the quantum dots by the gold nanoparticles with different sizes ( $3 \mathrm{~nm}, 15 \mathrm{~nm}$ and $80 \mathrm{~nm}$ ); (a) Fluorescence emission spectra of the solutions containing the DNA-quantum dots and the DNA-gold nanoparticles after addition of various concentrations (0 to $60 \mathrm{nM}$ ) of $\mathrm{Hg}$ (II) ions; (b) Normalized fluorescence emission intensity as a 
function of $\mathrm{Hg}$ (II) ions; (c) Stern-Volmer plots showing the efficiencies of quenching by three types of gold nanoparticles with different sizes.

As compared with the $15 \mathrm{~nm}$ and $80 \mathrm{~nm}$ sized gold nanoparticles, the $3 \mathrm{~nm}$ gold nanoparticle had a smaller quenching efficiency because of its smaller size and the lack of LSPR involvement. Although controversy still exists around its correctness, the theory of Perrson [63], now referred to as nanometal surface energy transfer (NSET), has been highly successful in describing the fluorescence quenching by small gold nanoparticles [23]. Unlike FRET, NSET does not require a resonant electronic transition, but rather an interband electronic transition. Persson's theory accounts for the formation of dipole-induced electron-hole pairs in a thin layer of a semi-infinite metal hemisphere. It considers the collective interaction of all the dipoles in a thin film near the surface of the metal, creating a $1 / d$ coupling instead of the usual $1 / d^{3}$ coupling of the dipole approximation. The distance dependence of the quenching in NSET is more than double the detectable distance in FRET, becoming very popular as a detection scheme. Although the theory is derived by assuming a semi-infinite surface, the quantum dots and dyes are of a similar scale in this experiment. This means that the surface of the gold nanoparticle is limited in size and will only accept a limited amount of energy. In short, for $3 \mathrm{~nm}$ sized gold nanoparticles, the energy transfer is limited to the NSET mechanism which can only accept energy by the formation of electron-hole pairs near the surface. The 15 and $80 \mathrm{~nm}$ gold nanoparticles are expected to have an increased energy interaction because of the existence of their LSPR absorption.

\subsection{Energy transfer mechanism in different sized gold systems}


As mentioned above, the different sized gold nanoparticles show distinct quenching efficiency, and existence of LSPR will affect the energy transfer rate. To further understand and verify the mechanism of energy transfer from the QDs to the gold nanoparticles, we have examined the separation distance $\left(d_{0}\right)$ at which the energy transfer efficiency is $50 \%$, and the power law $(n)$ for quenching efficiency $(E)$. For the NSET mechanism, $d_{0}$ can be quantified as [64]

$$
d_{0(N S E T)}=\left[0.225 \cdot \frac{\Phi_{Q D}}{\omega_{Q D}^{2}} \cdot \frac{1}{\omega_{F} k_{F}} \cdot c^{3}\right]^{1 / 4}
$$

where $\Phi_{Q D}$ is the quantum yield of the donor in the absence of acceptor, $\omega_{Q D}$ and $c$ represent the angular frequency of the donor emission and the light velocity in vacuum, $\omega_{F}=1.2 \times 10^{10} \mathrm{~m}^{-1}$ and $k_{F}=8.4 \times 10^{15} \mathrm{rad} / \mathrm{s}$ are the bulk gold angular frequency and Fermi vector, respectively. The $d_{0}$ value in the NSET was calculated to be $5.77 \mathrm{~nm}$ from equation 6.1 for all the gold nanoparticles.

In the FERT mechanism, $d_{0}$ can be described as [61]

$$
d_{0(F R E T)}=0.211\left[\kappa^{2} \Phi_{Q D}(n)^{-4} \cdot J(\lambda)\right]^{1 / 6}
$$

where $\kappa^{2}$ describes the relative orientation in space of the transition dipoles of the donor and the acceptor, and is taken to be $2 / 3$ assuming rapid orientational averaging of the donor within the lifetime of its excited state [65], $n$ is the refractive index of the medium (to be 1.33 in the present work), $J(\lambda)$ is the overlap integral between the normalized donor emission and the acceptor extinction coefficient. The overlap integral between the emission spectrum of the quantum dot and the extinction spectrum of the gold nanoparticle increases as the gold particle size increases. The values of the overlap integral are $J(\lambda)=5.5 \times 10^{15} \mathrm{M}^{-1} \cdot \mathrm{cm}^{-1} \cdot \mathrm{nm}^{4}, 3.9 \times 10^{18} \mathrm{M}^{-1} \cdot \mathrm{cm}^{-1} \cdot \mathrm{nm}^{4}$ and $6.6 \times 10^{21} \mathrm{M}^{-1} \cdot \mathrm{cm}^{-1} \cdot \mathrm{nm}^{4}$ for $3 \mathrm{~nm}, 15 \mathrm{~nm}$ and $80 \mathrm{~nm}$ gold nanoparticles. The $d_{0}$ values in the 
FRET model were thus $3.74 \mathrm{~nm}, 8.16 \mathrm{~nm}$ and $14.08 \mathrm{~nm}$ for the $3 \mathrm{~nm}, 15 \mathrm{~nm}$ and $80 \mathrm{~nm}$ gold nanoparticles.

(a)

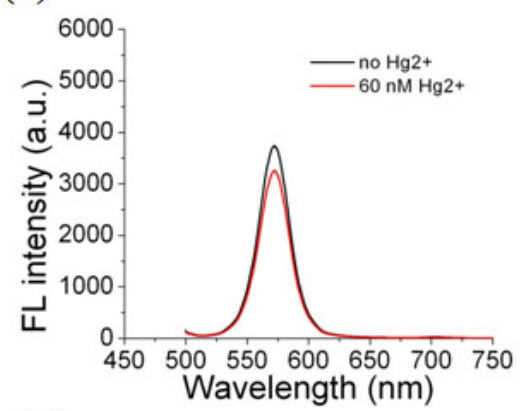

(c)

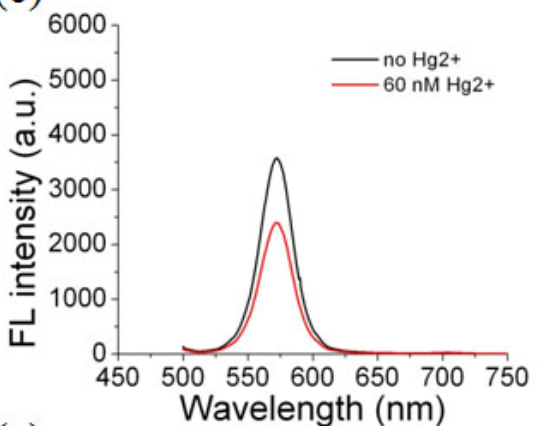

(e)

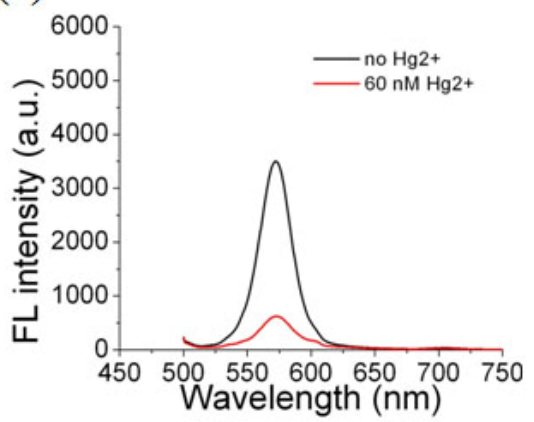

(b)

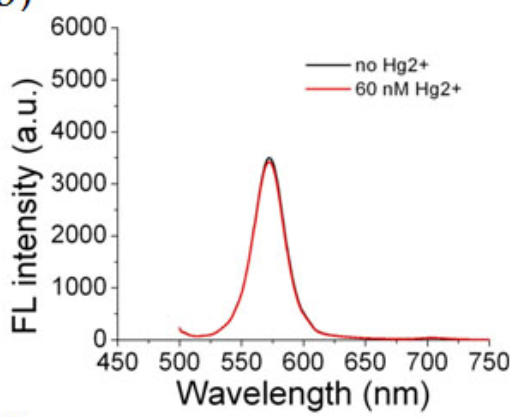

(d)

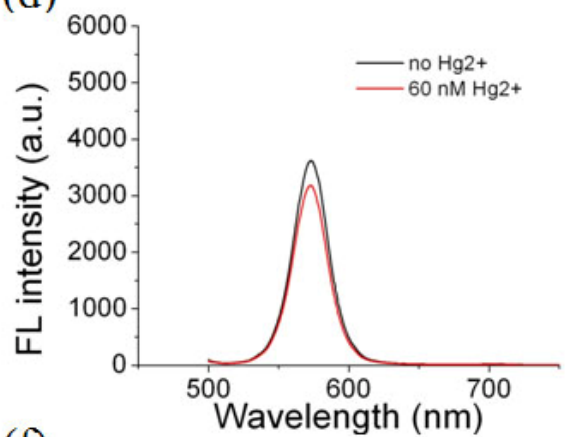

(f)

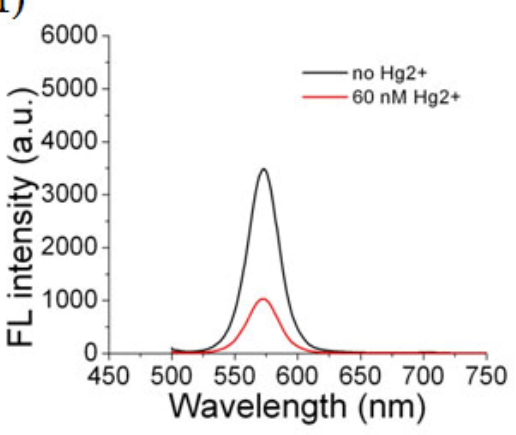

Figure 6.12 Fluorescence emission spectra of solutions of $\mathrm{CdSe} / \mathrm{ZnS}$ quantum dots and $3 \mathrm{~nm}$ gold nanoparticles when it uses DNA sequence of (a) 20 base pairs and (b) 30 base pairs, $15 \mathrm{~nm}$ gold nanoparticles when it uses DNA sequence of (c) 20 base pairs and (d) 30 base pairs, and 80 nm gold nanoparticles when it uses DNA sequence of (e) 20 base pairs and (f) 30 base pairs in the absence and presence of $60 \mathrm{nM} \mathrm{Hg}^{2+}$. 
A generic form of the efficiency of quenching follows a power law [64]

$$
E=\frac{1}{1+\left(d / d_{0}\right)^{n}}
$$

where $E$ is the quenching efficiency and $d$ is the separation distance between the surface of the gold nanoparticle and the donor [30], $n$ is dependent on the mechanism of energy transfer from the donor to the acceptor. $n=6$ for both the FRET model and $n=4$ for the NSET model. Taking the $d_{0}$ values calculated from the above, the theoretical plots of the quenching efficiency versus the separation distance were fitted following equation 6.4 (Figure 6.13). In our experiment, the length of double-stranded DNA between the QD and gold nanoparticle was varied to be 6.24, 9.64 and $13.04 \mathrm{~nm}$. The quenching efficiency was then measured in the three different separation distances (Figures 6.12). Comparing the experimental data of the quenching efficiency with the theoretical curves in Figure 6.13, it can be seen that the NSET model shows a better fit to the experimental data for the $3 \mathrm{~nm}$ gold particles. This is due to the lack of a pronounced LSPR dipole absorption necessary for FRET. The $1 / d^{4}$ dependence allows a longer detection distance than that with FRET. This is in agreement with the previous reports $[13,19,23,64]$. The FRET model provides a better fit to the experimental data for the $15 \mathrm{~nm}$ and $80 \mathrm{~nm}$ gold nanoparticles. This is to be expected because both have spectral overlap between the emission of quantum dots and the LSPR absorption of gold nanoparticles. The coupling between two dipoles leads to a $1 / d^{6}$ distance dependence of the quenching efficiency as it is usually seen in the conventional FRET $[66,67]$. 
(a)

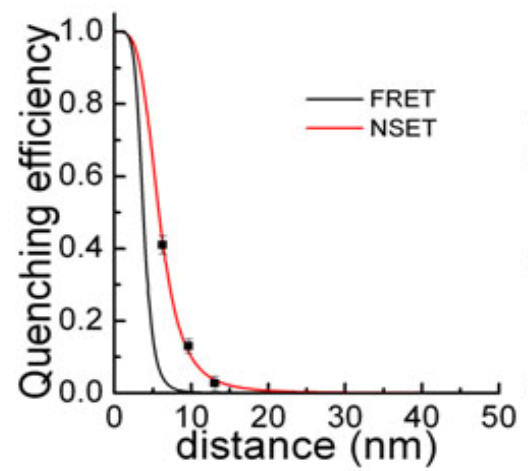

(b)

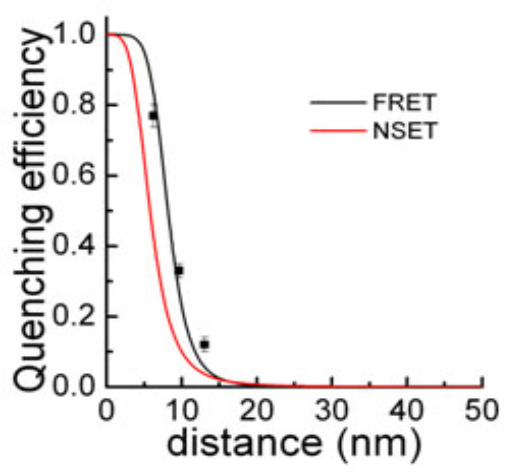

(c)

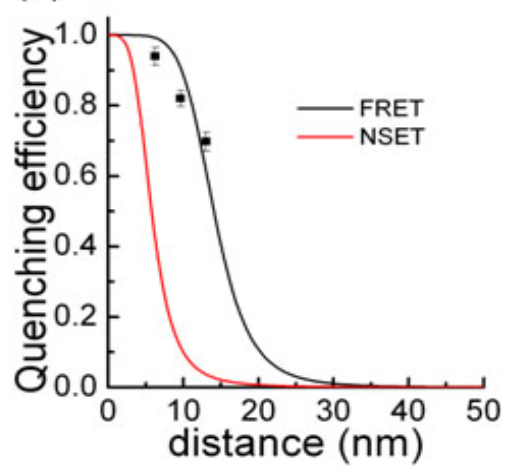

Figure 6.13 Experimental data points of the quenching efficiency and theoretical curves of the quenching efficiency versus the separation distance that were fitted based on the FRET and NSET models for (a) $3 \mathrm{~nm}$ gold nanoparticles, (b) $15 \mathrm{~nm}$ gold nanoparticles and (c) $80 \mathrm{~nm}$ gold nanoparticles.

\subsection{Summary}

In summary, the chapter firstly describes the synthesis of CdSe/ZnS QDs, and its size-tunable optical properties (optical absorption and fluorescence emission). Then, water-soluble QDs for biocompatible applications were obtained through the ligand exchange by MPA. Based on these, the energy transfer between quantum dots and gold nanoparticles with different sizes was investigated. The efficiency of fluorescence quenching of the quantum dots by the gold nanoparticles increases with an increase in the size of gold nanoparticles. It was found that the 3 $\mathrm{nm}$ gold nanoparticle without observable LSPR absorption quenched the fluorescence emission of quantum dots via the NSET mechanism, following a $1 / d^{4}$ distance-dependence. Large sized gold nanoparticles with significant LSPR followed the FRET mechanism of resonant energy transfer with a $1 / d^{6}$ distance-dependence. The $80 \mathrm{~nm}$ sized gold nanoparticles exhibit higher 
quenching efficiency than the $15 \mathrm{~nm}$ sized gold nanoparticle, which is attributed to an increase of the spectral overlap between the LSPR absorption and the emission of quantum dots. The $3 \mathrm{~nm}$ gold nanoparticle did not display a LSPR absorption and the quenching by the NSET mechanism was not as efficient as the FRET.

\section{References}

[1] Nolan, E. M.; Lippard, S. J. Chem. Rev., 2008, 108, 3443-3480.

[2] Rasnik, I.; McKinney, S. A.; Ha, T. Acc. Chem. Res., 2005, 38, 542-548.

[3] Brown, M. D.; Suteewong, T.; Kumar, R. S. S.; D’Innocenzo, V.; Petrozza, A.; Lee, M. M.;

Wiesner, U.; Snaith, H. J. Nano Lett., 2011, 11,438-445.

[4] Wang, Y.; Xie, X.; Goodson, T. Nano Lett., 2005, 5, 2379-2384.

[5] Lu, S.; Lingley, Z.; Asano, T.; Harris, D.; Barwicz, T.; Guha, S.; Madhukar, A. Nano Lett., 2009, 9, 4548-4552.

[6] Medintz, I. L.; Clapp, A. R.; Mattoussi, H.; Goldman, E. R.; Fisher, B.; Mauro, J. M. Nat. Mater., 2003, 2, 630-638.

[7] Clapp, A. R.; Medintz,I. L.; Mauro, J. M.; Fisher, B. R.; Bawendi, M. G.; Mattoussi, H. J. Am. Chem. Soc., 2004, 126, 301-310.

[8] Lakowicz, J. R. Principles of Fluorescence Spectroscopy, $3^{\text {rd }}$ ed.; Springer: Singapore, 2006.

[9] Goldman, E. R.; Medintz, I. L.; Whitley, J. L.; Hayhurst, A.; Clapp, A. R.; Uyeda, H. T.; Deschamps, J. R.; Lassman, M. E.; Mattoussi, H. J. Am. Chem. Soc., 2005, 127, 6744-6751.

[10] Gill, R.; Willner, I.; Shweky, I.; Banin, U. J. Phys. Chem. B, 2005, 109, 23715-23719.

[11] Medintz, I. L.; Farrell, D.; Susumu, K.; Trammell, S. A.; Deschamps, J. R.; Brunel, F. M.; Dawson, P. E.; Mattoussi, H. Anal. Chem., 2009, 81, 4831-4839. 
[12] Roberti, M. J.; Morgan, M.; Menéndez, G.; Pietrasanta, L. I.; Jovin, T. M.; Jares-Erijman, E. A. J. Am. Chem. Soc., 2009, 131, 8102-8107.

[13] Yun, C. S.; Javier, A.; Jennings, T.; Fisher, M.; Hira, S.; Peterson, S.; Hopkins, B.; Reich, N. O.; Strouse, G. F. J. Am. Chem. Soc., 2005, 127, 3115-3119.

[14] Reil, F.; Hohenester, U.; Krenn, J. R.; Leitner, A. Nano Lett., 2008, 8, 4128-4133.

[15] Zhang, J.; Fu, Y.; Chowdhury, M. H.; Lakowicz, J. R. J. Phys. Chem. C, 2007, 111, 1178411792.

[16] Pustovit, V. N.; Shahbazyan, T. V. Phys. Rev. B, 2011, 83, 085427.

[17] Viste, P.; Plain, J.; Jaffiol, R.; Vial, A.; Adam, P. M.; Royer, P. ACS Nano, 2010, 4, 759764.

[18] Rainó, G.; Stöferle, T.; Park, C.; Kim, H.-C.; Topuria, T.; Rice, P. M.; Chin, I.-J.; Miller, R. D.; Mahrt, R. F. ACS Nano, 2011, 5, 3536-3541

[19] Pons, T.; Medintz, I. L.; Sapsford, K. E.; Higashiya, S.; Grimes, A. F.; English, D. S.; Mattoussi, H. Nano Lett., 2007, 7, 3157-3164.

[20] Gueroui, Z.; Libchaber, A. Phys. Rev. Lett., 2004, 93, 166108.

[21] Kondon, M.; Kim, J.; Udawatte, N.; Lee, D. J. Phys. Chem. C, 2008, 112, 6695-6699.

[22] Bates, M.; Blosser, T. R.; Zhuang, X. Phys. Rev. Lett., 2005, 94, 108101.

[23] Jennings, T. L.; Singh, M. P.; Strouse, G. F. J. Am. Chem. Soc., 2006, 128, 5462-5467.

[24] Liu, X.; Wu, N. Q.; Wunsch, B. H.; Barsotti, R. J.; Stellacci, F. Small, 2006, 2, 1046-1050.

[25] Zhao, H.; Chaker, M.; Wu N. Q.; Ma, D. J. Mater. Chem., 2011, 21, 8898-8904.

[26] Tan, G. L.; Hömmerich, U.; Temple, D.; Wu, N. Q.; Zheng, J. G.; Loutts, G. Scripta Materialia, 2003, 48, 1469-1474. 
[27] Lu, Z. S.; Guo, C. X.; Yang, H. B.; Qiao, Y.; Guo, J.; Li, C. M. J. Colloid Interf. Sci., 2011, $353,588-592$.

[28] Ray, P. C.; Fortner, A.; Darbha, G. K. J. Phys. Chem. B, 2006, 110, 20745-20748.

[29] Gersten, J. I.; Nitzan, A. Surf. Sci., 1985, 158, 165-189.

[30] Griffin, J.; Singh, A. K.; Senapati, D.; Rhodes, P.; Mitchell, K.; Robinson, B.; Yu, E.; Ray, P. C. Chem. Eur. J., 2009, 15, $342-351$.

[31] Li, J. J.; Wang, Y. A.; Guo, W.; Keay, J. C.; Mishima,T. D.; Johnson, M. B.; Peng, X. J. Am. Chem. Soc., 2003, 125, 12567-12575.

[32] Dezhurov, S. V.; Volkova, I. Y.; Wakstein, M. S. Bioconjugate Chem., 2011, 22, 338-345.

[33] Pong, B.-K.; Trout, B. L.; Lee, J.-Y. Langmuir, 2008, 24, 5270-5276.

[34] Wuister, S. F.; Swart, I.; van Driel, F.; Hickey, S. G.; Donegá, C. de M. Nano Lett., 2003, 3, 503-507.

[35] Susumu, K.; Uyeda, H. T.; Medintz, I. L.; Pons,T.; Delehanty, J. B.; Mattoussi, H. J. Am. Chem. Soc., 2007, 129, 13987-13996.

[36] Chen, S.; Kimura, K. Langmuir, 1999, 15, 1075-1082.

[37] Ojea-Jiménez, I.; Puntes, V. J. Am. Chem. Soc., 2009, 131, 13320-13327.

[38] Basu, S.; Pande, S.; Jana, S.; Bolisetty, S.; Pal, T. Langmuir, 2008, 24, 5562-5568.

[39] Storhoff, J. J.; Elghanian, R.; Mucic, R. C.; Mirkin, C. A.; Letsinger, R. L. J. Am. Chem. Soc., 1998, 120, 1959-1964.

[40] Mitchell, G. P.; Mirkin, C. A.; Letsinger, R. L. J. Am. Chem. Soc., 1999, 121, 8122-8123.

[41] Maxwell, D. J.; Taylor, J. R.; Nie, S. J. Am. Chem. Soc., 2002, 124, 9606-9612.

[42] Yu, W. W.; Qu, L.; Guo, W.; Peng, X. Chem. Mater., 2003, 15, 2854-2860. 
[43] Huang, C. C.; Chiang, C. K.; Lin, Z. H.; Lee, K. H.; Chang, H. T. Anal. Chem., 2008, 80, 1497-1504.

[44] Jin, R.; Wu, G.; Li, Z.; Mirkin, C. A.; Schatz, G. C. J. Am. Chem. Soc., 2003, 125, 16431654.

[45] Dabbousi, B. O.; Rodriguez-Viejo, J.; Mikulec, F. V.; Heine, J. R.; Mattoussi, H.; Ober, R.; Jensen, K. F.; Bawendi, M. G. J. Phys. Chem. B, 1997, 101, 9463-9475.

[46] Qu, L.; Peng, Z. A.; Peng, X. Nano Lett., 2001, 1, 333-337.

[47] Kuçur, E.; Ziegler, J.; Nann, T. small, 2008, 4, 883-887.

[48] Murray, C. B.; Noms, D. J.; Bawendi, M. G. J. Am. Chem. Soc., 1993, 115, 8706-8715.

[49] Xie, R.; Kolb, U.; Li, J.; Basché, T.; Mews, A. J. Am. Chem. Soc., 2005, 127, 7480-7488.

[50] Li, J. J.; Wang, Y. A.; Guo, W.; Keay, J. C.; Mishima, T. D.; Johnson, M. B.; Peng, X. J. Am. Chem. Soc., 2003, 125, 12567-12575.

[51] Wu, N.; Fu, L.; Su, M.; Aslam, M.; Wong, K. C.; Dravid, V. P. Nano Lett., 2004, 4, 383386.

[52] Pi, F.; Kaneko, F.; Iwahashi, M.; Suzuki, M.; Ozaki, Y. J. Phys. Chem. B, 2011, 115, 62896295.

[53] Tong, H.-J.; Yu, J.-Y.; Zhang, Y.-H.; Reid, J. P. J. Phys. Chem. A, 2010, 114, 6795-6802.

[54] Dixit, S. K.; Goicochea, N. L.; Daniel, M.-C.; Murali, A.; Bronstein, L.; De, M.; Stein, B.; Rotello, V. M.; Kao, C. C.; Dragnea, B. Nano Lett., 2006, 6, 1993-1999.

[55]. Jiang, W.; Mardyani, S.; Fischer, H.; Chan, W. C. W. Chem. Mater., 2006, 18, 872-878.

[56] Kalasad, M. N.; Rabinal, M. K.; Mulimani, B. G. Langmuir, 2009, 25, 12729-12735.

[57] Kim, J.; Lee, D. J. Am. Chem. Soc., 2007, 129, 7706-7707.

[58] Dulkeith, E.; Ringler, M.; Klar, T. A.; Feldmann, J. Nano Lett., 2005, 5, 585-589. 
[59] Freeman, R.; Finder, T.; Willner, I. Angew. Chem. Int. Ed., 2009, 48, 1-5.

[60] Haas, C.; Kräling, K.; Cichon, M.; Rahe, N.; Carell, T. Angew. Chem., 2004, 116, 1878 1880.

[61] Lakowicz, J. R. Principles of Fluorescence Spectroscopy, 3rd ed.; Spinger Academic: New York, 2006.

[62] Shopa, M.; Kolwas, K.; Derkachova, A.; Derkachov, G. Opto-Electr. Rev., 2010, 18, 421428.

[63] Persson, B. N. J.; Lang, N. D. Phys. Rev. B, 1982, 26, 5409-5415.

[64] Singh M. P.; Strouse, G. F. J. Am. Chem. Soc., 2010, 132, 9383-9391.

[65] Dale, R. E.; Eisinger, J.; Blumberg, W. E. Biophys. J., 1979, 26, 161-193.

[66] Chowdhury, M. H.; Gray, S. K.; Pond, J.; Geddes, C. D.; Aslan, K.; Lakowicz, J. R. J. Opt. Soc. Am. B, 2007, 24, 2259-2267.

[67] Zhang, J.; Fu, Y.; Lakowicz, J. R. J. Phys. Chem. C, 2007, 111, 50-56. 


\section{CHAPTER 7: MERCURY(II) DETECTION BASED ON ENERGY TRANSFER BETWEEN QUANTUM DOTS AND GOLD NANOPARTICLES}

\subsection{Introduction}

With the advancement of human civilization and demands of high-quality living level, growing concern for the food safety, environmental pollution and detection of counterfeiting, etc. has sparked interest in developing reliable and effective sensing/monitoring approaches. In Chapters 4 and 5, we demonstrated that SERS is useful for a wide range of applications, relying on the localized surface plasmon resonance (LSPR) absorption along with the substrates. The proper LSPR substrate can promote performances of SERS devices. Furthermore, Chapter 6 systematically describes particle size-effect on the energy transfer between $\mathrm{CdSe} / \mathrm{ZnS}$ core/shell quantum dots (QDs) and gold nanoparticles. The different particle sizes result in distinct energy transfer mechanism: nanometal surface energy transfer (NSET) and classical Förster (also called as fluorescence) resonance energy transfer (FRET). Although both energy transfers possess the common physical origin in essence, the apparent characters are different. The NSET follows a $1 / d^{4}$ separation dependence while the FRET follows a $1 / d^{6}$ separation dependence, which is mainly attributed to involvement of LSPR absorption. In this chapter, we focus on the application of energy transfer between QDs and gold nanoparticles in $\mathrm{Hg}^{2+}$ detection.

$\mathrm{Hg}^{2+}$ is one of the most toxic heavy metals and a kind of persistent contaminant that is not biodegradable and thus is retained in the ecosystem [1-3]. Moreover, $\mathrm{Hg}^{2+}$ can accumulate in 
vital organs through the food chain and cause human illness and dysfunctional, posing a threat to human health, animals, plants, and the planet itself [4-8]. Conventional analytical techniques including cold-vapor atomic fluorescence spectrometry (CV-AFS) [9], cold-vapor atomic absorption spectrometry (CV-AAS) [10], inductively coupled plasma-mass spectroscopy (ICPMS) [11], ultraviolet-visible spectrometry and X-ray absorption spectroscopy [12] have been extensively used for measurement of $\mathrm{Hg}^{2+}$ in water samples. However, these methods are laborintensive, time-consuming, laboratory-based and require large sample volumes. Furthermore, they cannot be used for on-site detection of $\mathrm{Hg}^{2+}$ in the environment. Therefore, there is a strong incentive to develop a sensitive, reliable and convenient approach for monitoring $\mathrm{Hg}^{2+}$ in the environment. Much effort has been made to develop portable sensors, including fluorescent sensors based on organic fluorophores and conjugated polymers, scanometric sensors and colorimetric sensors [13-17]. Special attention has been paid to the sensors based on Förster resonance energy transfer (FRET) between a fluorescent donor and an acceptor [18-22]. Generally, organic dyes are used as the energy donor and the energy acceptor to construct FRET sensors in previous studies. Nevertheless, organic dyes usually undergo some disadvantages such as degradation and low fluorescence efficiency.

Recently, the organic energy acceptor was replaced with a gold nanoparticle although the energy donor still was the organic dye, leading to a high energy transfer rate from the organic energy donor to the gold nanoparticle acceptor and a long quenching distance [23-28]. It would be of significance to eliminate the organic dyes that are present in the above-mentioned FRET and NSET sensors, because the use of organic dyes results in photo-bleaching, low sensitivity and poor reproducibility. 
It was introduced in Chapter 6 that semiconductor quantum dots (QDs) are attractive fluorescent labels due to their high quantum efficiency, photo-stability and size-tunable optical properties as compared to conventional organic dyes [29-31]. In the present work, we used QDs as the energy donors and $3 \mathrm{~nm}$ gold nanoparticles as the energy acceptors to construct a nanosurface energy transfer (NSET) sensor for $\mathrm{Hg}^{2+}$ detection in water. It is found that this kind of sensor provides high sensitivity, selectivity and strong anti-inference capability for $\mathrm{Hg}^{2+}$ detection.

\subsection{Materials and experimental methods}

\subsubsection{Materials}

Cadmium oxide (CdO, 99.99+\%), zinc oxide ( $\mathrm{ZnO}, 99.99 \%)$, sulfur (powder, 99.98\%), trioctylphosphine oxide (TOPO, tech. 90\%), 1-octadecene (ODE, tech. 90\%), oleic acid (tech. 90\%), octyldecylamine (ODA, tech. 90\%), trioctylphosphine (TOP, tech. 90\%), selenium (powder, 99+\%), p-mercaptobenzoic acid (MBA, tech. 90\%), 3-mercaptopropionic acid (MPA, 99+\%), $\mathrm{Cu}\left(\mathrm{NO}_{3}\right)_{2}, \mathrm{Hg}\left(\mathrm{NO}_{3}\right)_{2},\left(\mathrm{HgNO}_{3}\right)_{2} \cdot 2 \mathrm{H}_{2} \mathrm{O}, \mathrm{Pb}\left(\mathrm{NO}_{3}\right)_{2}(99.995 \%), \mathrm{Cd}\left(\mathrm{NO}_{3}\right)_{2} \cdot 4 \mathrm{H}_{2} \mathrm{O}$, and $5 \mathrm{M}$ $\mathrm{NaCl}$ stock solution were purchased from Sigma-Aldrich. n-tetradecylphosphonic acid (TDPA) was purchased from PCI Synthesis. Methylene dichloride $\left(\mathrm{CH}_{2} \mathrm{Cl}_{2}, \mathrm{HPLC}\right.$ grade), methanol (ACS grade) and acetone (ACS grade) were purchased from Fisher Scientific. Chloroauric acid trihydrate $\left(\mathrm{HAuCl}_{4} \cdot 3 \mathrm{H}_{2} \mathrm{O}\right), \mathrm{NaBH}_{4}(98 \%), \mathrm{KI}(99.9 \%), \mathrm{AgNO}_{3}$ (Premion, 99.995\%) and ethylenediamine (99\%) were obtained from Alfa-Aesar. All chemicals and solvents were obtained from the commercial sources and used directly without any further purification, and all glassware were cleaned successively with $\mathrm{HNO}_{3}$ and D.I. water, and then dried before use. 


\subsubsection{Synthesis of DNA-QD conjugates and DNA-gold conjugates}

Firstly, we synthesized QDs with desired fluorescence emission wavelength according to the described method in Section 6.2. Briefly, a mixture of $0.0514 \mathrm{~g} \mathrm{CdO}, 0.2232 \mathrm{~g}$ TDPA, $1.0 \mathrm{~g}$ ODA and $2.5 \mathrm{~g}$ TOPO was heated to $300{ }^{\circ} \mathrm{C}$ above under a Ar flow to obtain a colorless clear solution, and then lowered to $260{ }^{\circ} \mathrm{C}$ for the injection of TOPSe solution $(0.0665 \mathrm{~g}$ Se in $2.4 \mathrm{~mL}$ TOP). The temperature was kept at $240{ }^{\circ} \mathrm{C}$ for the growth of the desired CdSe nanocrystals. The $0.1 \mathrm{M}$ Zn-containing stock solution was made by $0.407 \mathrm{~g} \mathrm{ZnO}$ in $20 \mathrm{~mL}$ oleic acid and $30 \mathrm{~mL}$ ODE. The $0.1 \mathrm{M}$ S-containing stock solution was made by $0.16 \mathrm{~g} \mathrm{~S}$ in $50 \mathrm{~mL}$ ODE. Once the CdSe nanocrystals with the desired fluorescence emission was observed, the temperature was lowered to about $220{ }^{\circ} \mathrm{C}$, and the calculated amount of the $\mathrm{Zn}$ - or S-stock solutions for each $\mathrm{ZnS}$ monolayer were alternately injected. After that, the resulting $\mathrm{CdSe} / \mathrm{ZnS}$ nanocrystals were purified by methanol and acetone, followed by the ligand exchange with MPA to prepare the water soluble QDs.

The $3 \mathrm{~nm}$ gold nanoparticles were prepared through the reduction of $\mathrm{HAuCl}_{4} \cdot 3 \mathrm{H}_{2} \mathrm{O}$ by $\mathrm{NaBH}_{4}$ [32]. The water soluble QDs and gold nanoparticles were functionalized by two complementary ssDNA except for three deliberately designed T-T mismatches $\left(5^{\prime}\right.$-HS- $\left(\mathrm{CH}_{2}\right)_{6}{ }^{-}$ CAGTTTGGAC-3' and 5'-HS-( $\left(\mathrm{CH}_{2}\right)_{6}$-GTCCTTTCTG-3'), respectively [33, 34].

The loading of ssDNA onto the QDs was determined based on the UV-vis absorption spectra before and after modification with ssDNA and the absorbance difference at $260 \mathrm{~nm}$ [35]. The molar extinction coefficient $(\varepsilon)$ of QDs is $2.0 \times 10^{5} \mathrm{~L} /(\mathrm{mol} \cdot \mathrm{cm})$ according to the literature [36]. In addition, the exact DNA concentration can be determined by the absorbance at $260 \mathrm{~nm}$, according to [37]

$$
1 \text { O.D. }(260 \mathrm{~nm})=50 \mathrm{ng} \mathrm{DNA} / \mu \mathrm{L}
$$


Therefore, since the resulting DNA-QD stock solution has a QD concentration of $0.9 \mu \mathrm{M}$ $\left(C=A_{551 \mathrm{~nm}} /(b \cdot \varepsilon)\right)$, the average DNA loading can be calculated to be four DNAs per QD.

Similarly, we can obtain the DNA loading per gold nanoparticle. $3 \mathrm{~nm}$ gold nanoparticles contain 976 gold atom $\left(\mathrm{Au}_{976}\right)$ [38]. The average DNA loading was roughly estimated to be one DNA per gold nanoparticle. In the above calculation, we used the molecular weight $3043 \mathrm{~g} / \mathrm{mol}$ and $2985 \mathrm{~g} / \mathrm{mol}$ for 5'-HS-( $\left(\mathrm{CH}_{2}\right)_{6}$-CAGTTTGGAC-3' and 5'-HS-( $\left.\mathrm{CH}_{2}\right)_{6}$-GTCCTTTCTG-3', respectively.

The average DNA loadings of the resulting DNA-functionalized QDs and gold nanoparticles (Probe A: QD-S-CAGTTTGGAC-3' and Probe B: Au-S-GTCCTTTCTG-3') were four DNAs per QD and one DNA per Au NP based on the optical absorption spectra.

\subsubsection{Assembly of QD-DNA-gold ensemble and $\mathrm{Hg}^{2+}$ detection}

The DNA-QD stock solution was diluted to $30 \mathrm{~mL}$ using the PBS buffer. Afterwards, $8 \mathrm{~mL}$ diluted DNA-QD solution, $4 \mathrm{~mL} 0.3 \mathrm{M}$ PBS solution $\left(10 \mathrm{mM} \mathrm{NaH} \mathrm{PO}_{4} / \mathrm{Na}_{2} \mathrm{HPO}_{4}\right.$ and $0.3 \mathrm{M}$ $\mathrm{NaCl}$ ) and $0.5 \mathrm{~mL}$ DNA-Au NPs were mixed, followed by addition of $10 \mathrm{mM}$ ethylenediamine PBS stock solution and $5 \mathrm{M} \mathrm{NaCl}$. This resulted in the test solution.

The test was conducted in a PBS solution that contained 96 nM DNA-QDs, 104 nM DNAgold nanoparticles, $0.1 \mathrm{mM}$ ethylenediamine, $10 \mathrm{mM} \mathrm{NaH}{ }_{2} \mathrm{PO}_{4} / \mathrm{Na}_{2} \mathrm{HPO}_{4}$ and $0.3 \mathrm{M} \mathrm{NaCl}$.

After $\mathrm{Hg}^{2+}$ ions were added into the test solution $\left(1 \mu \mathrm{M} \mathrm{Hg}^{2+}\right.$, the time-dependent fluorescence emission intensity at $572 \mathrm{~nm}$ was monitored to determine the hybridization kinetics.

For the sensitivity measurement, different concentrations of $\operatorname{Hg}^{2+}(0,1,2,3,4,5,6,810,15$, $20,30,40,60,80,100,200,500$ and $1000 \mathrm{nM}$ ) were added. The fluorescence emission spectra were then monitored. 
For the selectivity measurement, various different metal ions of $100 \mathrm{nM}$ was supplied with their salts $\left(\mathrm{KI}, \mathrm{AgNO}_{3},\left(\mathrm{HgNO}_{3}\right)_{2} \cdot 2 \mathrm{H}_{2} \mathrm{O}, \mathrm{CaCl}_{2}, \mathrm{Cd}\left(\mathrm{NO}_{3}\right)_{2} \cdot 4 \mathrm{H}_{2} \mathrm{O}, \mathrm{Cu}\left(\mathrm{NO}_{3}\right)_{2}, \mathrm{Hg}\left(\mathrm{NO}_{3}\right)_{2}\right.$ and $\left.\mathrm{Pb}\left(\mathrm{NO}_{3}\right)_{2}\right)$. The fluorescence emission spectra were monitored after the induction of metal ions into the test assay.

The river water sample was taken from the Monongahela River, and then filtered with the filter paper (Millipore, Isopore membrane). Then, $8 \mathrm{~mL}$ DNA-QD solution, $4 \mathrm{~mL}$ filtered river water and $0.5 \mathrm{~mL}$ DNA-Au NP solution were mixed, followed by the measurement of various concentrations of $\mathrm{Hg}^{2+}$ according to the same procedure as shown above.

\subsubsection{Characterization}

The size of QDs and gold nanoparticles was observed under a transmission electron microscope (TEM) with a JEM 2100F operated at $200 \mathrm{kV}$. The TEM specimens were prepared by dropping the gold nanoparticle solution onto a carbon-coated copper grid, and then dried in air. UV-visible absorption spectra were recorded in the range of $200-800 \mathrm{~nm}$ with the Shimadzu UV-2550 spectrometer (Japan). The fluorescence (FL) emission spectra were measured using a HITACHI F-7000 fluorescence spectrophotometer. X-ray photoelectron spectra (XPS) measurements were carried out with PHI 5000 Versa Probe system (Physical Electronics, MN).

The obtained spectra were calibrated with the reference to the $\mathrm{C} 1 \mathrm{~s}$ peak of aliphatic carbon at $284.8 \mathrm{eV}$.

\subsection{Design of energy transfer-based sensors and its operation principle}


Figure 7.1a shows the optical absorption and fluorescence emission spectra of TOPO-QDs used in the present work. Inset indicates the strong yellow fluorescence emission. TEM image indicates a uniform size distribution with $3.8 \mathrm{~nm}$ in diameter (Figure 7.1b).

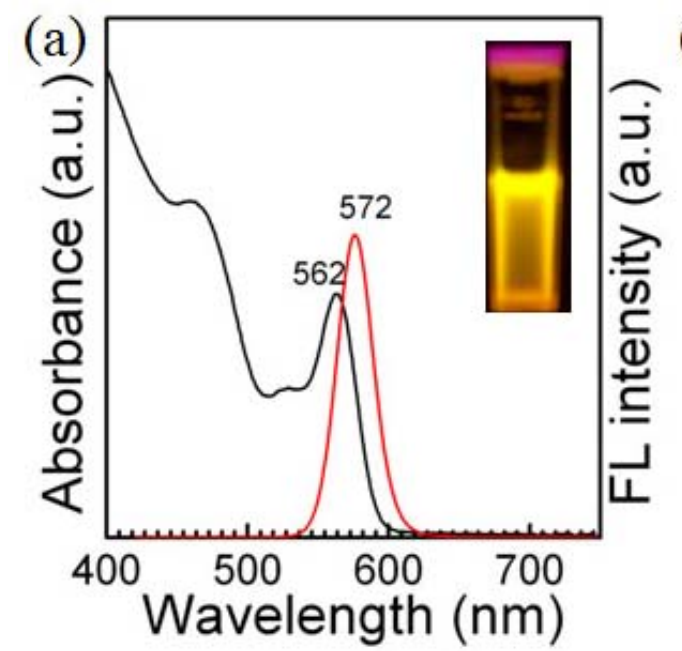

(b)

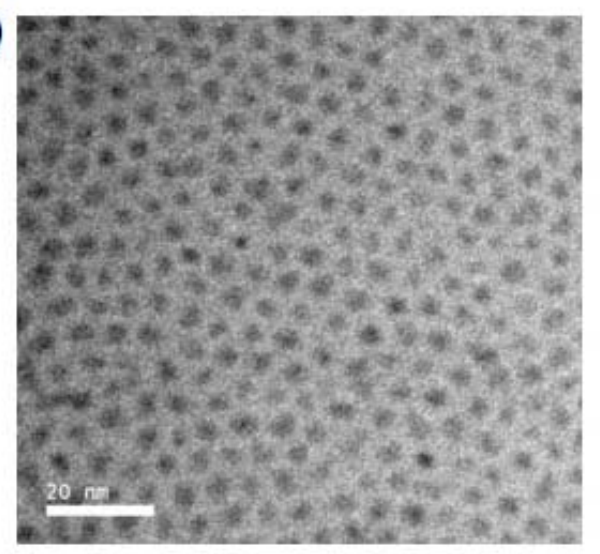

Figure 7.1 (a) optical absorption and fluorescence emission spectra of TOPO-CdSe/ZnS quantum dots in $\mathrm{CH} 2 \mathrm{Cl} 2$. Inset shows the optical photo of TOPO-CdSe/ZnS quantum dots in $\mathrm{CH}_{2} \mathrm{Cl}_{2}$ under excitation of $365 \mathrm{~nm}$ laser. (b) TEM image of TOPO-CdSe/ZnS quantum dots.

We used the present QDs as the energy donors and $3 \mathrm{~nm}$ gold nanoparticles as the energy acceptors to construct $\mathrm{Hg}^{2+}$ sensor. Figure 7.2a schematically shows the design of the NSET sensor. Firstly, two probes (Probe A: QD-S-CAG TTT GGA C-3' and Probe B: Au-S-G TCC TTT CTG-3') were prepared by functionalization of the $3.8 \mathrm{~nm}$ sized water-soluble CdSe/ZnS core-shell QDs and the $3 \mathrm{~nm}$ sized gold nanoparticles with ssDNA sequences of 5'-HS- $\left(\mathrm{CH}_{2}\right)_{6}{ }^{-}$ CAG TTT GGA C-3' and 5'-HS- $\left(\mathrm{CH}_{2}\right)_{6}-\mathrm{G}$ TCC TTT CTG-3', respectively. These two ssDNA strands are complementary except for three deliberately designed T-T mismatches. In the 
(a)

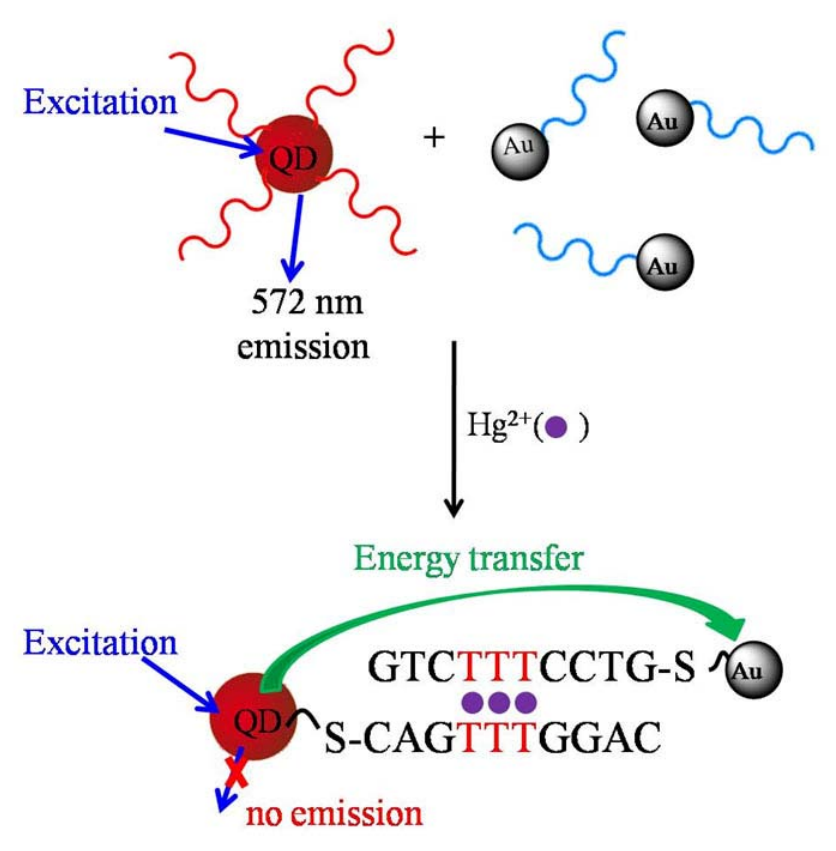

(b)

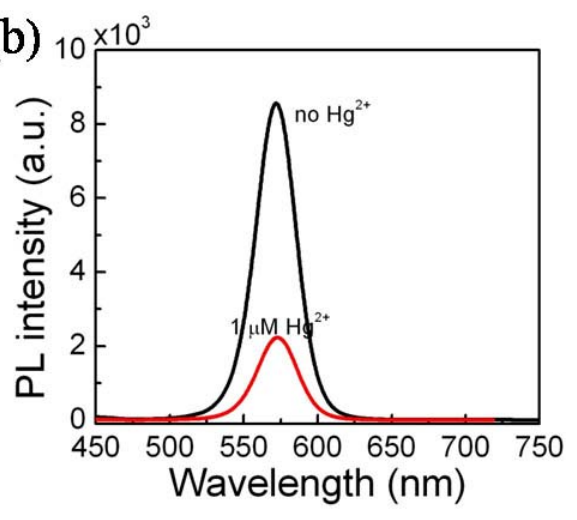

(c)

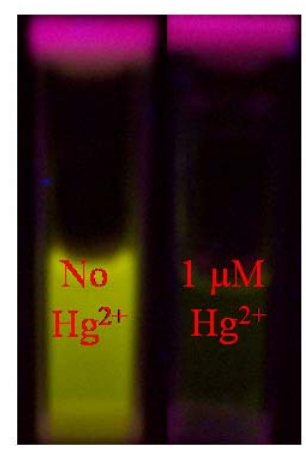

Figure 7.2 (a) Schematic illustration of the operating principle of the QD/DNA/gold nanoparticle ensemble sensor for $\mathrm{Hg}^{2+}$ detection. (b) Fluorescence emission spectra and (c) Photograph under $365 \mathrm{~nm}$ laser excitation of the QD/DNA/gold nanoparticle solution (96 nM QDs, $104 \mathrm{nM}$ gold nanoparticles and $0.1 \mathrm{mM}$ ethylenediamine in $0.3 \mathrm{M}$ PBS) before and after addition of $1 \mu \mathrm{M} \mathrm{Hg}^{2+}$.

absence of $\mathrm{Hg}^{2+}$, Probe A and Probe B are not capable of hybridization and dispersed in the aqueous solution due to the electrostatic repulsion from negatively charged ssDNA attached on the nanoparticle surfaces. In this case, Probe A will fluoresce at $572 \mathrm{~nm}$ under laser excitation. When $\mathrm{Hg}^{2+}$ ions are present, DNA hybridization will occur due to the formation of thymine$\mathrm{Hg}^{2+}$-thymine $\left(\mathrm{T}-\mathrm{Hg}^{2+}-\mathrm{T}\right)$ complexes [39]. As a result, QDs and gold nanoparticles will be brought into close proximity, which enables NSET between the QDs and gold nanoparticles, 
leading to quenching of the fluorescence emission of the QDs (Figure 7.2a,b). Visual examination showed that the brightness of the QD/DNA/gold nanoparticle solution diminished remarkably after addition of $1 \mu \mathrm{M} \mathrm{Hg}^{2+}$ ion (Figure 7.2c). The further experiment indicated that there is no observable change in the fluorescence emission intensity when $60 \mathrm{nM}$ or more $\mathrm{Hg}^{2+}$ was added to the solution only containing QDs but not gold nanoparticles (Figure 7.3). Therefore, quenching of fluorescence emission of QDs is due to gold nanoparticles rather than addition of $\mathrm{Hg}^{2+}$ ions.

Generally, the fluorescence emission of the quantum dots could be quenched by the proximal gold nanoparticle via two possible mechanisms, that is, charge transfer (such as Dexter $[40,41]$ ) and energy transfer (such as FRET [18-22] and NSET [23-28]). In the Dexter mechanism, charge carriers are exchanged between the donor and the acceptor, which requires the overlap of the wavefunctions between the donor and the acceptor. The rate of charge transfer decreases exponentially as the distance, following the relation of $\exp [-(2 r / L)]$, where $r$ is the edge-to-edge distance between the donor and the acceptor, $L$ is the sum of the van-der Waals radii of the donor and the acceptor. Therefore, the rate of charge transfer drops to negligibly small values as the edge-to-edge distance increases more than one or two molecular diameters $(0.5 \sim 1 \mathrm{~nm})$. FRET originates from the dipole-dipole interaction, which requires the overlap of the emission band of the donor with the absorption band of the acceptor. The rate of energy transfer depends on the $1 / d^{6}$ separation distance. The fluorescence emission of the donor could be quenched in the separation distance up to $10 \mathrm{~nm}$. NSET does not require a resonant interaction between the electrons but rather an interband electronic transition, which is described by the theory of Perrson [42]. The rate of energy transfer decreases with a $1 / d^{4}$ distance-dependence. NSET typically shows longer energy transfer distance than FRET. Willner et al claimed that the 
quenching of quantum dots (QDs) was ascribed to the electron transfer from the QD to metallic ions [35]. Based on the investigation in Chapter 6, it is believed that the fluorescence emission of the quantum dots was quenched by the gold nanoparticle via the NSET mechanism. Our results show that the efficiency of quenching followed a $1 / d^{4}$ distance-dependence instead of the exponential relation. The fluorescence emission of the quantum dots was quenched by the gold nanoparticle at the separation distance up to $\sim 6.2 \mathrm{~nm}$, which was much longer than the typical quenching distance in the Dexter mechanism.

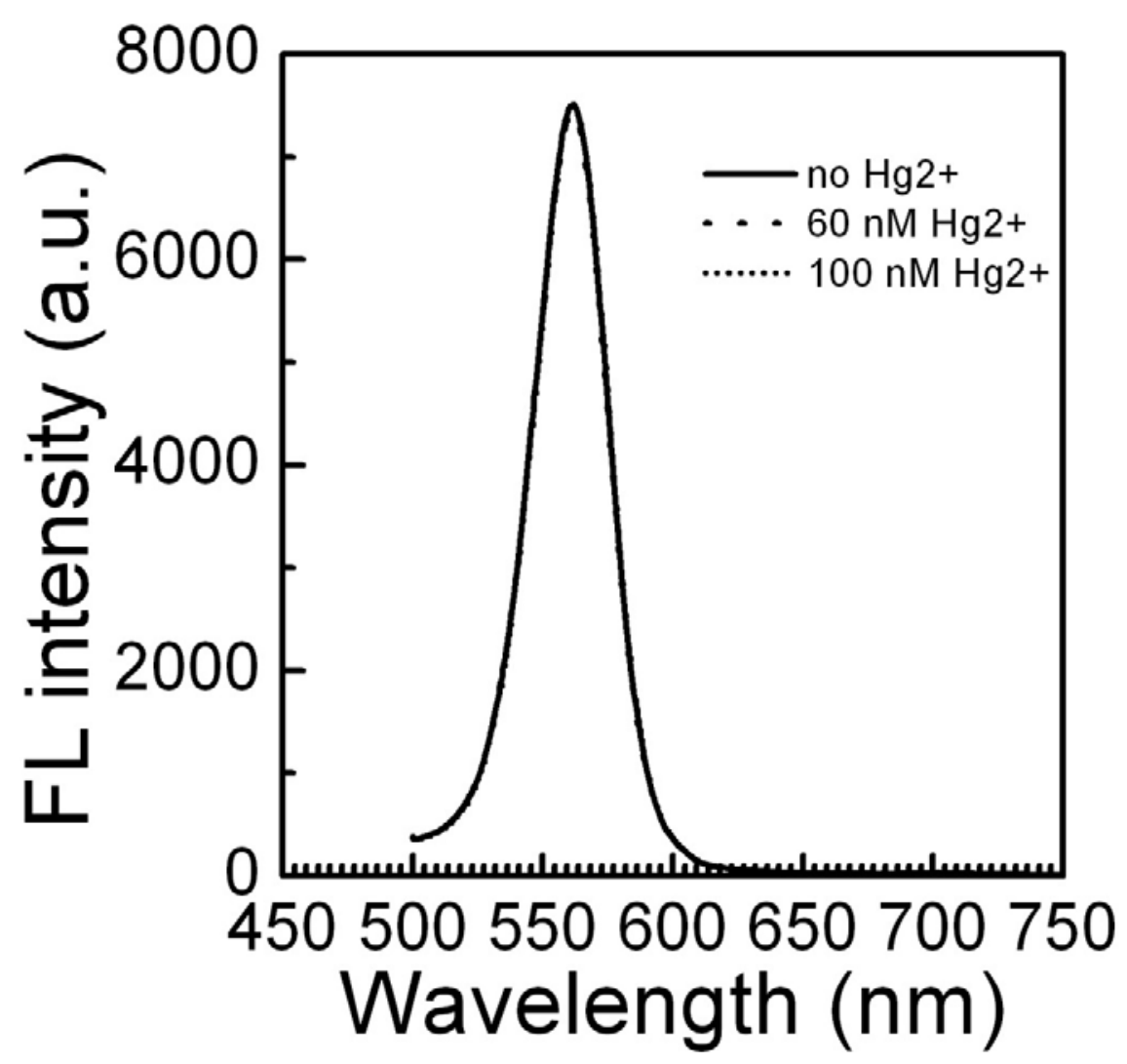

Figure 7.3 Fluorescence emission spectra of DNA-CdSe/ZnS quantum dots in the absence and presence of $60 \mathrm{nM}$ and $100 \mathrm{nM}$. 


\subsection{Hybridization kinetics}

The hybridization kinetics of Probe A and Probe B was monitored in the phosphate buffered saline (0.3 M PBS) solution ( $\mathrm{pH}=7.0)$ containing $10 \mathrm{mM} \mathrm{NaH} \mathrm{PO}_{4} / \mathrm{Na}_{2} \mathrm{HPO}_{4}$ and $0.3 \mathrm{M} \mathrm{NaCl}$ with the presence of $1 \mu \mathrm{M} \mathrm{Hg}^{2+}$ at room temperature (Figure 7.4). It was found that it took about 10 min to accomplish DNA hybridization through the coordinating interaction between $\mathrm{Hg}^{2+}$ and the thymine base. The assay time was shorter than the results reported previously [34].

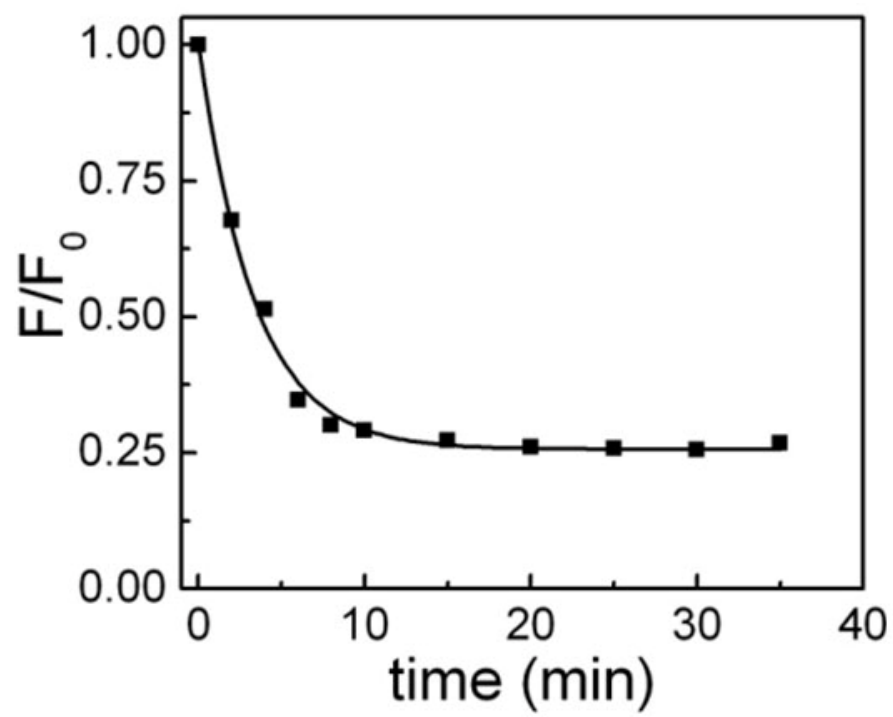

Figure 7.4 Time-dependent fluorescence emission intensity at $572 \mathrm{~nm}$ of the QD/DNA/gold nanoparticle system at $1 \mu \mathrm{M} \mathrm{Hg}^{2+}$ concentration of (96 nM CdSe/ZnS QDs, $104 \mathrm{nM}$ gold nanoparticles and $0.1 \mathrm{mM}$ ethylenediamine in $0.3 \mathrm{M}$ PBS).

\subsection{Sensitivity testing toward $\mathrm{Hg}($ II) concentration}

To evaluate the sensitivity of the NSET sensor, the fluorescence emission intensity of the assay was measured after addition of various concentrations of $\mathrm{Hg}^{2+}$ ions $(0,1,2,3,4,5,6,8$, 
$10,15,20,30,40,60,80,100,200,500$ and $1000 \mathrm{nM}$ ) (Figure 7.5). As shown in Figure 7.5a,b, the intensity of fluorescence emission was very sensitive to the change in the $\mathrm{Hg}^{2+}$ concentration, and decreased as an increase in the $\mathrm{Hg}^{2+}$ concentration. The fluorescence quenching efficiency was quantified by the equation $\left(\eta=\left(\mathrm{F}_{\mathrm{o}}-\mathrm{F}\right) / \mathrm{F}_{\mathrm{o}}\right)$, where $\mathrm{F}_{\mathrm{o}}$ and $\mathrm{F}$ were the fluorescence intensity at $572 \mathrm{~nm}$ before and after $\mathrm{Hg}^{2+}$ addition, respectively. A linear correlation between the fluorescence quenching efficiency and the $\mathrm{Hg}^{2+}$ concentration was observed in the $\mathrm{Hg}^{2+}$ concentration range from $2 \mathrm{nM}$ to $60 \mathrm{nM}$ (Figure 7.5c). The international World Health Organization (WHO) and the U.S. Environmental Protection Agency (EPA) regulate the maximum allowable levels of $\mathrm{Hg}^{2+}$ in drinking water to be $6 \mathrm{ppb}$ and $2 \mathrm{ppb}$, respectively. The present method enables the efficient detection of $\mathrm{Hg}^{2+}$ in water with an ultra-low limit of detection (LOD) of $2 \mathrm{nM}(0.4 \mathrm{ppb})$, which is lower than both WHO and EPA standards. The high sensitivity is mainly attributed to the efficiency fluorescence quenching by coupling of several gold nanoparticles to one QD. The LOD of the present NSET mercury sensor was improved in comparison with those of the previously reported colorimetric sensor $(\mathrm{LOD}=3 \mu \mathrm{M})[16]$ and the FRET sensor $(\mathrm{LOD}=40 \mathrm{nM})$ [39] although all these three types of sensors have the similar molecular recognition probe, i.e., the DNA containing the $\mathrm{T}-\mathrm{Hg}^{2+}-\mathrm{T}$ coordination. 

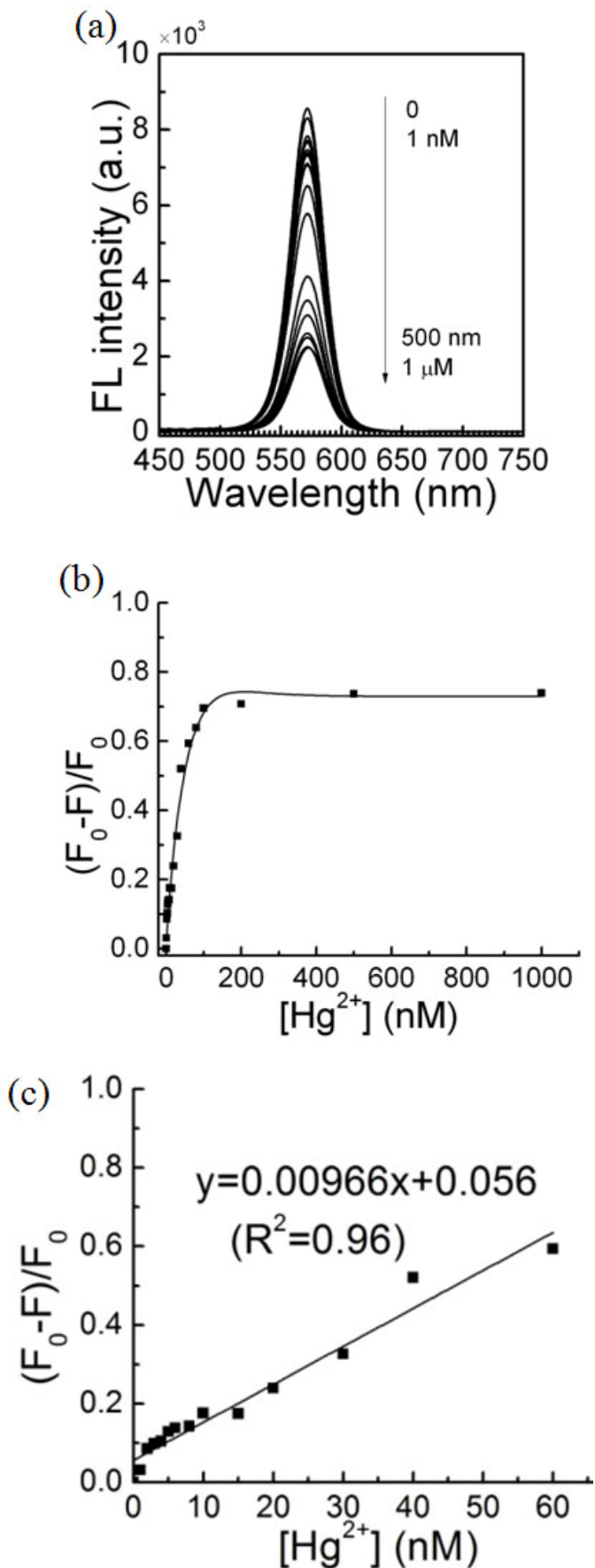

Figure 7.5 (a) Fluorescence emission spectra of the QD/DNA/Au NP system in 0.3 M PBS solution after addition of various concentrations of $\mathrm{Hg}^{2+}(0,1,2,3,4,5,6,8,10,15,20,30,40$, $60,80,100,200,500$ and $1000 \mathrm{nM}$ ) into the $0.3 \mathrm{M}$ PBS solution (96 nM QDs, $104 \mathrm{nM}$ Au NPs 
and $0.1 \mathrm{mM}$ ethylenediamine). (b) Plot of fluorescence quenching efficiency as a function of the $\mathrm{Hg}^{2+}$ concentration. (c) linear region of (b).

\subsection{Selectivity testing toward metal ions}

The selectivity of the NSET sensor has been investigated by testing the fluorescence quenching efficiency in the presence of other environmental metal ions, including $\mathrm{K}^{+}, \mathrm{Ag}^{+}, \mathrm{Hg}^{+}$, $\mathrm{Ca}^{2+}, \mathrm{Cd}^{2+}, \mathrm{Cu}^{2+}$ and $\mathrm{Pb}^{2+}$. At first, the fluorescence quenching efficiency was measured upon addition of a single type of $100 \mathrm{nM}$ metal ions into the $0.3 \mathrm{M}$ PBS solution (Figure 7.6). The result showed excellent selectivity toward $\mathrm{Hg}^{2+}$ over other metal ions including $\mathrm{Hg}^{+}$. The excellent selectivity for $\mathrm{Hg}^{2+}$ was attributed to its specific chelating ability with DNA through the formation of $\mathrm{T}-\mathrm{Hg}^{2+}-\mathrm{T}$ complexes [39]. Furthermore, to test the anti-interference capability, the fluorescence quenching efficiency of the assay was measured in the PBS solution in which $\mathrm{Hg}^{2+}$ co-existed with the mixture of $\mathrm{K}^{+}, \mathrm{Ag}^{+}, \mathrm{Hg}^{+}, \mathrm{Ca}^{2+}, \mathrm{Cd}^{2+}, \mathrm{Cu}^{2+}$ and $\mathrm{Pb}^{2+}$ ions (the concentration of each metal ion in the mixture was $100 \mathrm{nM}$ ) (Figure 7.6). Although the addition of the mixed metal ions without $\mathrm{Hg}^{2+}$ resulted in little higher fluorescence quenching than that of the individual metal ion, the fluorescence quenching efficiency was obviously far higher in the presence of $\mathrm{Hg}^{2+}$. This indicated that the present nanosensor had strong anti-interference capability and excellent selectivity. 


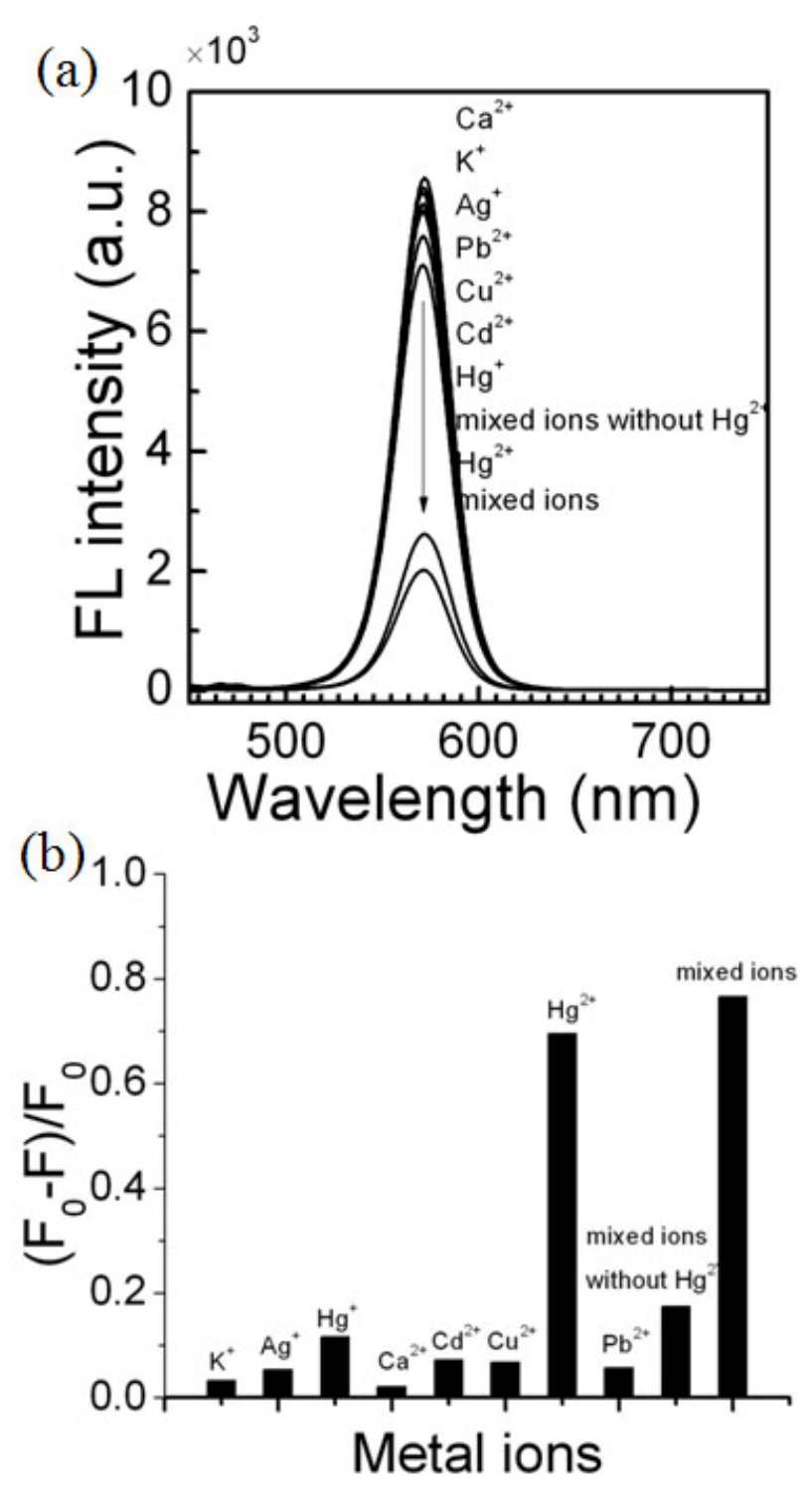

Figure 7.6 Fluorescence quenching efficiency in the presence of various metal ions. The concentration of each metal ion is $100 \mathrm{nM}$ (96 nM QDs, $104 \mathrm{nM} \mathrm{Au} \mathrm{NPs}$ and $0.1 \mathrm{mM}$ ethylenediamine in $0.3 \mathrm{M}$ PBS).

7.7 Anti-interference testing in river water 
In order to test whether the NSET sensor is able to be used in the real-world water, the interference of unknown substance in the river water on the fluorescent emission of nanosensor was evaluated with the water sample from the Monongahela River near our campus. Prior to testing, the river water was filtered by the filter paper (Millipore, Isopore membrane). The total mercury content in the filtered river water was measured to be $<0.1 \mathrm{ppb}$ by Agilent $7500 \mathrm{ce}$ inductively coupled plasma mass spectrometry (ICP-MS). The filtered water was then mixed with the stock DNA-QD buffer solution in the ratio of 1:2 $(\mathrm{v} / \mathrm{v})$ to obtain the DNA-QD test solution. The sensitivity of the QD/DNA/gold/river water system was checked after addition of various concentrations of $\mathrm{Hg}^{2+}$ ions $(0,1,2,4,6,8,10,15,20,30,40,60,80,100,200,500$ and $1000 \mathrm{nM})$. The initial fluorescence intensity in the DNA-QD solution containing the river water was much lower than that in the pure buffer solution used (Figure 7.7a), which was believed to be ascribed to the fluorescence quenching of the QDs by the various environmental substances in the river water. The fluorescence intensity of the assay decreased as an increase in the concentration of the added $\mathrm{Hg}^{2+}$ ions (Figure 7.7b). The sensitivity (the slope of the linear region) was a little lower and the limit of detection increased to $6 \mathrm{nM}(1.2 \mathrm{ppb})$ as compared to that $(0.4 \mathrm{ppb})$ of the pure PBS assay (Figure $7.8 \mathrm{c})$. 

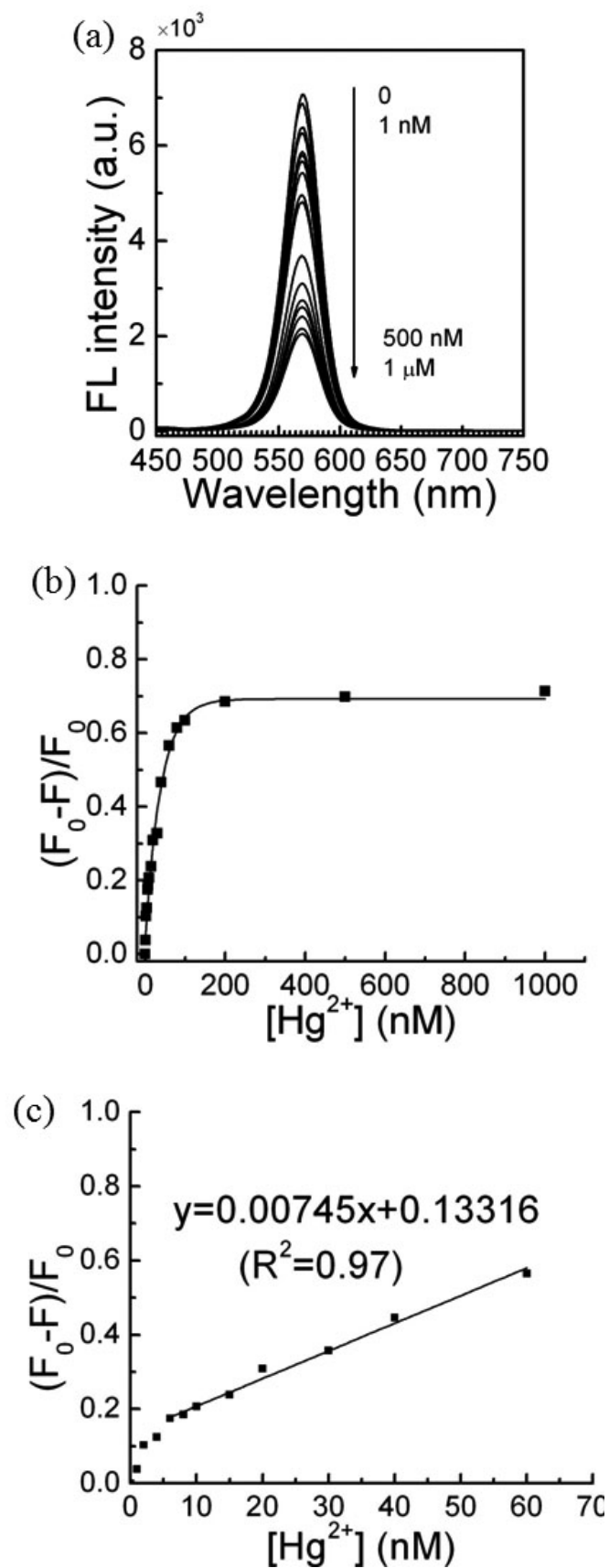

Figure 7.7 (a) Fluorescence emission spectra of the QD/DNA/Au NP system in river water containing various concentrations of $\mathrm{Hg}^{2+}(0,1,2,4,6,8,10,15,20,30,40,60,80,100,200$, 500 and $1000 \mathrm{nM}$ ) (96 nM QDs, $104 \mathrm{nM} \mathrm{Au} \mathrm{NPs,} 0.1 \mathrm{mM}$ ethylenediamine and $0.3 \mathrm{M} \mathrm{NaCl}$ in 
the mixture of the PBS with the river water). (b) Plot of the fluorescence quenching efficiency as a function of the $\mathrm{Hg}^{2+}$ concentration in river water. (c) linear region of (b).

\subsection{Summary}

This chapter is engaged in developing an ultrasensitive and highly selective nanosensor for $\mathrm{Hg}^{2+}$ detection in water, which is based on the NSET in the QD/DNA/gold nanoparticle ensemble. This sensor shows a limit of detection of $0.4 \mathrm{ppb}$ in the buffer solution. The excellent selectivity toward $\mathrm{Hg}^{2+}$ was demonstrated in an aqueous solution in the presence of other environmental metal ions. The developed nanosensor can be used for on-site detection of mercury in real aquatic environment. The developed nanosensor has a number of distinct advantages. First, the QD-gold optical assay eliminates the photo-bleaching problem that is usually associated with organic dyes. Second, individual QDs are bright fluorophores due to their high quantum yield, which enables the QD/gold assay to offer high sensitivity. Third, the QD/DNA/gold ensemble enables the NSET sensing mechanism. It has been reported that NSET has higher energy transfer efficiency and wider energy transfer distance than the conventional FRET $[23,43,44]$. Fourth, DNA as a molecular recognition probe is more stable in a nonphysiological solution than enzymes, proteins and live microbes that are typically used as molecular recognition probes in biosensors. Finally, the design of our nanosensor system provides a potential capability of simultaneous and discriminative detection of several metal ions in a multi-analyte sample through tuning the optical emission of quantum dots at various wavelengths. The fluorescence emissions of the multi-color QDs can be excited with a single laser at a wavelength far from the emission wavelengths of all the QDs. This is a significant advantage over the multiple light sources required to excite a series of organic dyes. 


\section{References}

[1] Nolan, E. M.; Lippard, S. J. Chem. Rev., 2008, 108, 3443-3480.

[2] Balaji, T.; El-Safty, S. A.; Matsunaga, H.; Hanaoka, T.; Mizukami, F. Angew. Chem. Int. Ed., 2006, 45, 7202-7208.

[3] Yilmaz, F.; Özdemir, N.; Demirak, A.; Tuna, A. L. Food Chem., 2007, 100, 830-835.

[4] Wang, Z.; Zhang, D.; Zhu, D. Anal. Chim. Acta, 2005, 549, 10-13.

[5] Huang, C.-C.; Yang, Z.; Lee, K.-H.; Chang, H.-T. Angew. Chem. Int. Ed., 2007, 46, 6824 6828.

[6] Darbha, G. K.; Singh, A. K.; Rai, U. S.; Yu, E.; Yu, H.; Ray, P. C. J. Am. Chem. Soc. 2008, 130, 8038-8043.

[7] Ramesh, G. V.; Radhakrishnan, T. P. ACS Appl. Mater. Interfaces, 2011, 3, 988-994.

[8] Pu, K.-Y.; Luo, Z.; Li, K.; Xie, J.; Liu, B. J. Phys. Chem. C, 2011, 15, 13069-13075.

[9] Lorber, K. E. Waste Manage. Res., 1986, 4, 3-13.

[10]. Kunkel, R.; Manahan, S. E. Anal. Chem., 1973, 45, 1465-1468.

[11]. Bings, N. H.; Bogaerts, A.; Broekaert, J. A. C. Anal. Chem., 2006, 78, 3917-3946.

[12]. Bernaus, A.; Gaona, X.; Esbrí, J. M.; Higueras, P.; Falkenberg, G.; Valiente, M. Environ. Sci. Technol. 2006, 40, 4090-4095.

[13] Lee, J.; Jun, H.; Kim, J. Adv. Mater., 2009, 21, 1-4.

[14] Lee, J.; Mirkin, C. A. Anal. Chem., 2008, 80, 6805-6808.

[15]. Lee, J.; Han, M. S.; Mirkin, C. A. Angew. Chem. Int. Ed., 2007, 46, 4093-4096.

[16] Xue, X.; Wang, F.; Liu, X. J. Am. Chem. Soc., 2008, 130, 3244-3245.

[17] Li, D.; Wieckowska, A.; Willner, I. Angew. Chem. Int. Ed., 2008, 47, 3927 -3931.

[18] Roy, R.; Hohng, S.; Ha, T. Nat. Method, 2008, 5, 507-516. 
[19] Goldman, E. R.; Medintz, I. L.; Whitley, J. L.; Hayhurst, A.; Clapp, A. R.; Uyeda, H. T.; Deschamps, J. R.; Lassman, M. E.; Mattoussi, H. J. Am. Chem. Soc., 2005, 127, 6744-6751.

[20] Zhang, X.; Xiao, Y.; Qian, X. Angew. Chem. Int. Ed., 2008, 47, 8025-8029.

[21] Davis, J. J.; Burgess, H.; Zauner, G.; Kuznetsova, S.; Salverda, J.; Aartsma, T.; Canters, G. W. J. Phys. Chem. B, 2006, 110, 20649-20654.

[22] Ray, P. C.; Fortner, A.; Darbha, G. K. J. Phys. Chem. B, 2006, 110, 20745-20748.

[23] Yun, C.; Javier, S. A.; Jennings, T.; Fisher, M.; Hira, S.; Peterson, S.; Hopkins, B.; Reich, N. O.; Strouse, G. F. J. Am. Chem. Soc., 2005, 127, 3115-3119.

[24] Kondon, M.; Kim, J.; Udawatte, N.; Lee, D. J. Phys. Chem. C, 2008, 112, 6695-6699.

[25] Gill, R.; Willner, I.; Shweky, I.; Banin, U. J. Phys. Chem. B, 2005, 109, 23715-23719.

[26] Dyadyusha, L.; Yin, H.; Jaiswal, S.; Brown, T.; Baumberg, J. J.; Booy, F. P.; Melvin, T. Chem. Commun., 2005, 3201-3203.

[27] Darbha, G. K.; Ray, A.; Ray, P. C. ACS Nano, 2007, 1, 208-214.

[28] Ye, B.; Yin, B. Angew. Chem. Int. Ed., 2008, 47, 8386-8389.

[29]Talapin, D. V.; Rogach, A. L.; Kornowski, A.; Haase, M.; Weller, H. Nano Lett., 2001, 1, 207-211.

[30] Peng, X.; Schlamp, M. C.; Kadavanich, A. V.; Alivisatos, A. P. J. Am. Chem. Soc., 1997, $119,7019-7029$

[31] Kairdolf, B. A.; Smith, A. M.; Nie, S. J. Am. Chem. Soc., 2008, 130, 12866-12867.

[32] Chen, S.; Kimura, K. Langmuir, 1999, 15, 1075-1082.

[33] Mitchell, G. P.; Mirkin, C. A.; Letsinger, R. L. J. Am. Chem. Soc., 1999, 121, 8122-8123.

[34] Maxwell, D. J.; Taylor, J. R.; Nie, S. J. Am. Chem. Soc., 2002, 124, 9606-9612.

[35] Freeman, R.; Finder, T.; Willner, I. Angew. Chem. Int. Ed., 2009, 48, 7818-7821. 
[36] Yu, W. W.; Qu, L.; Guo, W.; Peng, X. Chem. Mater., 2003, 15, 2854-2860.

[37] Molecular Techniques and Methods, Construction of Genomic Library, http://www.molecularinfo.com/MTM/A/A2/A2-3/A2-3-1.html, (accessed on June 12, 2011).

[38] Hostetler, M. J.; Wingate, J. E.; Zhong, C.-J.; Harris, J. E.; Vachet, R. W.; Clark, M. R.; Londono, J. D.; Green, S. J.; Stokes, J. J.; Wignall, G. D.; Glish, G. L.; Porter, Marc D.; Evans, N. D.; Murray, R. W. Langmuir, 1998, 14, 17-30.

[39] Ono, A.; Togashi, H. Angew. Chem. Int. Ed., 2004, 43, 4300-4302.

[40] Dexter, D. L. J. Chem. Phys., 1953, 21, 836-850.

[41] Inokuti, M.; Hirayama, F. J. Chem. Phys., 1965, 43, 1978-1989.

[42] Persson, B. N. J.; Lang, N. D. Phys. Rev. B, 1982, 26, 5409-5415.

[43] Jennings, T. L.; Singh, M. P.; Strouse, G. F. J. Am. Chem. Soc., 2006, 128, 5462-5467.

[44] Haldar, K. K.; Sen, T.; Patra, A. J. Phys. Chem. C, 2010, 114, 4869-4874. 


\section{CHAPTER 8: CONCLUSIONS AND REMARKS ON FUTURE RESEARCH}

\subsection{Conclusions}

In this work, gold nanostructures were used to develop surface-enhanced Raman scattering (SERS)- and resonance energy transfer (RET)-based sensing platforms for chemical, biological and environmental applications. Gold nanomaterials with various sizes and shapes exhibit unique optical properties including LSPR frequency, intensity and field distribution, which render distinguishing SERS and RET efficiency, and hence result in different performances among SERS sensors or RET sensors based on these gold nanostructures.

Specifically, the present work draws conclusions as follows:

1. Molecular structures of Raman tags are of fundamental and practical importance in the design of SERS devices, which determines the amplification of Raman signals and thus the sensitivity of SERS detection. SERS difference among various structural molecules (MPA, Cys, TP and MBA) originates from the electronic interaction with the gold nanoparticles to some extent. It is demonstrated that MBA with benzene ring structure exhibits the strongest SERS than the linear MPA and Cys. Our results show that the electrons transfer from the gold nanoparticle to the surface-immobilized aromatic molecules. The transferred electrons in the aromatic molecule monolayers are delocalized and lead to enhancement of electromagnetic field surrounding the gold nanoaprticles. This type of EM enhancement can contribute to "hot spot", and amplify the SERS signal. 
2. Shape of gold nanoparticles affects not only the intensity and position of LSPR absorption but also the electric field distribution, and thus determines the SERS activity.

a). The gold nanostars show the highest SERS activity under both $532 \mathrm{~nm}$ and $785 \mathrm{~nm}$ incident lasers while the gold nanospheres have low SERS activity.

b). The three-dimension FDTD simulation shows the significant different electric field distributions among gold nanospheres, nanorods and nanostars. The gold nanostars exhibit the highest maximum electric field intensities in both cases of $532 \mathrm{~nm}$ and $785 \mathrm{~nm}$ laser excitations. In addition, the maximum electric field intensity appears around two ends of nanorods and around these tips of nanostars so that the sharp components in nanostructures can concentrate and confine the electromagnetic field. Therefore, the shape difference among these gold nanostructures governs the SERS activity difference.

3. Gold@MGITC@SiO $\mathrm{Sin}_{2}$ sandwiched structures were prepared. It was found that

a). These sandwiched structures can improve the colloidal stability and LSPR stability in a wide range of liquid environments (e.g. organic solvents and high ionic strength). More importantly, $\mathrm{SiO}_{2}$ encapsulation prevents MGITC molecules from leaking out and improves the stability of signals of Raman probe.

b). These unique advantages shown above enable the potential capability of this kind SERS sensors for applications in monitoring of DNA hybridization.

c). A SERS sensor for ATP detection is developed, which is based on the gold nanostar@MGITC@SiO $\mathrm{Si}_{2}$ sandwiched structure as the signaling probe and ATP-binding aptamer as the recognition element. This sensor exhibits a detection limit of $12.4 \mathrm{pM}$ and excellent 
selectivity over its analogues such as GTP and CTP. This high sensitivity is attributed to the strong signal amplification ability of gold nanostar@MGITC@ $\mathrm{SiO}_{2}$.

4. The high quality $\mathrm{CdSe} / \mathrm{ZnS}$ quantum dots were synthesized by a thermolytic route. The optical properties (absorption and fluorescence spectra) of $\mathrm{CdSe} / \mathrm{ZnS}$ quantum dots were successfully tailored by controlling the reaction temperature and reaction time. The emission wavelength can be tuned from $510 \mathrm{~nm}$ to $620 \mathrm{~nm}$. The fluorescent water soluble CdSe/ZnS quantum dots can be achieved by ligand exchange method with MPA.

5. The energy transfer between quantum dots and different sized gold nanoparticles was investigated. The fluorescence quenching efficiency of the quantum dots by the gold nanoparticles increases with an increase in the size of the gold nanoparticles. Furthermore, it was found that

a). $3 \mathrm{~nm}$ gold nanoparticles without observable LSPR absorption quenched the fluorescence emission of quantum dots via the nanometal suface energy transfer mechanism, which follows a $1 / d^{4}$ distance-dependence.

b). Large sized gold nanoparticles with significant LSPR quenched the fluorescence emission of quantum dots, following the Förster resonance energy transfer with a $1 / d^{6}$ distancedependence.

c). The large sized gold nanoparticles quench the fluorescence emission much effectively than the small sized gold nanoparticles, which is attributed to the effect of electromagnetic field enhancement due to the excitation of LSPR. Also, the increasing spectral overlap between the 
LSPR absorption and the emission of quantum dots contributes to the increasing quenching efficiency.

6. The QD/DNA/gold system is able to be applied for $\mathrm{Hg}^{2+}$ detection in robust aquatic environments. The developed NSET sensor with $3 \mathrm{~nm}$ gold nanoparticles as the quencher can realize the specific $\mathrm{Hg}^{2+}$ detection with high selectivity and a detection limit of $0.4 \mathrm{ppb}$ in a PBS solution. The excellent selectivity for $\mathrm{Hg}^{2+}$ ions over other environmental ions is attributed to the specific chelating ability with DNA through the formation of $\mathrm{T}-\mathrm{Hg}^{2+}-\mathrm{T}$ complexes. In addition, the anti-interference capability has been demonstrated because of specific detection of $\mathrm{Hg}^{2+}$ in the presence of various environmental ions. The developed QD/DNA/gold sensor can be also applied in the river water matrix for $\mathrm{Hg}^{2+}$ detection with a detection limit of $1.2 \mathrm{ppb}$.

\subsection{Remarks on future research}

Surface plasmon-enhanced phenomena (e.g. SERS and RET), as a subfield of nanophotonics, have emerged in recent several decades, and are reforming photovoltaic devices and analytical techniques with ultrasensitive detection in chemical and biological applications. Applications of these effects are still in its infancy, and experimental and theoretical investigations are in high demand to understand the underlying physical essence and to construct the applicable devices. Based on the present investigation and my understanding, I recommend the following work to be done in the future:

1. The results shown in Chapter 3 have indicated that charge transfer can occur from gold to the surface ligands with favorable electronic level, which contributes to the SERS enhancement. It is essential to theoretically investigate the underlying physical essence about the charge transfer 
between gold nanoparticles and surface molecules. What kind of molecular levels are needed to facilitate the electron transfer? What magnitude of electromagnetic contributions can be achieved by charge transfer? Theoretical investigations could clarify these questions and thus provide thorough understanding on the present work.

2. In Chapters 4 and 5, it was mentioned that encapsulation of a large number of MGITC molecules inside the $\mathrm{SiO}_{2}$ layer renders strong signal amplification ability and high sensitivity to the developed SERS sensor. Despite that it seems for sure, different MGITC amounts in the $\mathrm{SiO}_{2}$ layer should be performed and then the sensitivity of SERS sensors is checked.

3. Experiments have demonstrated that the ultrasensitive SERS sensor can be developed, which is based on the gold nanostar@MGITC@ $\mathrm{SiO}_{2}$ as the signaling probe. It was mentioned that SERS possesses facile potential multiplexing capability because of its unique spectral characteristics. Therefore, the future work should focus on the development of SERS sensors with multiplexing detection capability.

4. Chapter 6 has shown that different sized gold nanoparticles result in different energy transfer mechanisms (NSET and FRET). It is suggested that the enhanced energy transfer with the increasing size of gold nanoparticles is attributed to the enhanced electromagnetic field due to the excitation of localized surface plasmon resonance and the increasing spectral overlap between LSPR absorption and emission spectra. Theoretical simulation on effect of electromagnetic field on energy transfer will strengthen understanding of the underlying physical essence. It is also predicted that both NSET and FRET mechanisms exist in the energy transfer systems containing various sized gold nanoparticles. Theoretical simulation will show the contributions of NSET and FRET present in these systems. 


\section{PEER-REVIEWED PUBLICATION LIST}

1). Ming Li, Scott K. Cushing, Qiaoyi Wang, Xiaodong Shi, Lawrence A. Hornak, Zhanglian Hong, and Nianqiang Wu. Size-dependent energy transfer between CdSe/ZnS quantum dots and gold nanoparticles. The Journal of Physical Chemistry Letters, 2 (2011), 2125-2129.

2). Ming Li, Qiaoyi Wang, Xiaodong Shi, Lawrence A. Hornak, and Nianqiang Wu. Detection of mercury(II) by quantum dot-DNA-gold nanoparticle ensemble based nanosensor via nanometal surface energy transfer. Analytical Chemistry, 83 (2011), 7061-7065.

3). Ming Li, Rui Li, Chang Ming Li, and Nianqiang Wu. Electrochemical and optical biosensors based on nanomaterials and nanostructures: review. Frontiers in Bioscience, S3 (2011), 13081331. (invited review).

4). Ming Li, Scott K. Cushing, Jianming Zhang, Jessica Lankford, Zoraida P. Aguilar, Dongling Ma, and Nianqiang Wu. Shape-Dependent Surface-Enhanced Raman Scattering in Gold@Raman-Probe@Silica Sandwiched Nanoparticles for Biocompatible Applications. Nanotechnology, 23 (2012) 115501.

5). Ming Li, Scott K. Cushing, Guangwen Zhou, Jessica Lankford, Lawrence A. Hornak, and Nianqiang $\mathrm{Wu}$. Chemical Contribution to Electromagnetic Enhancement in Surface-Enhanced Raman Scattering. (to be submitted).

6). Ming Li, Jianming Zhang, Savan Suri, Letha J. Sooter, Dongling Ma, and Nianqiang Wu. Detection of Adenosine Triphosphate with an Aptamer Biosensor Based on Surface-Enhanced Raman Scattering. Analytical Chemistry, 2012, DOI: 10.1021/ac203325z.

7). Ming $\mathbf{L i}$, and Nianqiang Wu. Detection of $\mathrm{pH}$ Value through Surface-Enhanced Raman Scattering (SERS). ECS Transactions, 2012 (in press).

8). Jin Wang, De Nyago Tafen, James P. Lewis, Zhanglian Hong, Ayyakkannu Manivannan, Mingjia Zhi, Ming Li, and Nianqiang Wu. Origin of the Photocatalytic Activity of Nitrogendoped $\mathrm{TiO}_{2}$ Nanobelts. Journal of the American Chemical Society, 131 (2009), 12290-12227. 
9). Qian Zhang, Ting Sun, Feng Cao, Ming Li, Minghui Hong, Jikang Yuan, Qingyu Yan, Huey Hoon Hng, Nianqiang $\mathrm{Wu}$, and Xiaogang Liu. Tuning the shape and thermoelectric property of PbTe nanocrystals by bismuth doping. Nanoscale, 2 (2010), 1256-1259.

10). Huan Zhang, Weiqiang Ding, Kai He, and Ming Li. Synthesis and Characterization of Crystalline Silicon Carbide Nanoribbons. Nanoscale Research Letters, 5 (2010) 1264-1271. 